\title{
Desymmetrization of Prochiral Cyclopentenes Enabled by Enantioselective Palladium-Catalyzed Oxidative Heck Reaction
}

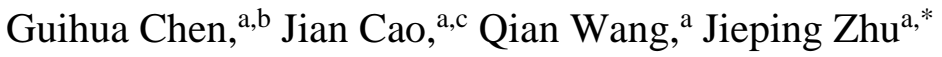

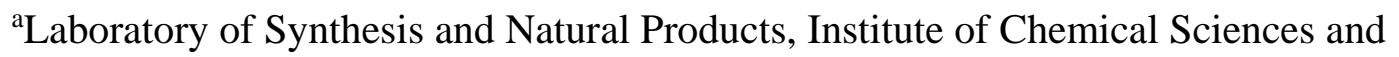 \\ Engineering, Ecole Polytechnique Fédérale de Lausanne, EPFL-SB-ISIC-LSPN, BCH 5304, \\ 1015 Lausanne, Switzerland \\ ${ }^{b}$ Key Laboratory of Applied Chemistry of Chongqing Municipality, School of Chemistry and \\ Chemical Engineering, Southwest University, Chongqing 400715, P. R. China \\ ${ }^{c}$ Key Laboratory of Organosilicon Chemistry and Material Technology of Ministry of \\ Education, Hangzhou Normal University, Hangzhou 311121, P. R. China
}

\section{Table of Contents}

General Information

Preparation and characterization data of substrates $\mathbf{1 a - 1 q}$

General procedure for catalytic asymmetric oxidative Heck reaction and characterization data of products 3aa-3qg

Scale up reactions and post-transformations S32

References . S37

Copies of NMR spectra S38

Copies of SFC traces. .589

Crystallographic data for $\mathbf{4}$ and $\mathbf{5}$ S115 


\section{General Information}

NMR spectra were recorded on AV2 400 Bruker spectrometers. Chemical shifts are given in ppm. The spectra are calibrated to the residual ${ }^{1} \mathrm{H}$ and ${ }^{13} \mathrm{C}$ signals of the solvents or TMS. Multiplicities are abbreviated as follows: singlet (s), doublet (d), triplet (t), doublet of doublets (dd), doublet of doublets of doublets (ddd), triplet of triplets (tt), doublet of triplets (dt), multiplet (m) and broad (br). Infrared spectra were recorded on a JASCO FT/IR-4100 spectrometer. The accurate masses were measured by the mass spectrometry service of the EPFL by ESI-TOF using a QTOF Ultima from Waters or APPI-FT-ICR using a linear ion trap Fourier transform ion cyclotron resonance mass spectrometer from Thermo Scientific. Optical rotations were obtained with a Jasco P-2000 polarimeter (589 nm). Enantiomeric excesses were determined with a Thar SFC Investigator system using chiral stationary phase columns by comparing the samples with the appropriate racemic ones, column and elution details are specified in each entry. Melting points were measured using a Stuart SMP30.

Materials and Methods: Unless otherwise stated, starting materials and chiral ligands were purchased from commercial sources (Aldrich, ABCR, Acros, Merck, Fluka and VWR international). More sensitive compounds were stored in a desiccator or in a glove-box if required. Solvents were purged with argon and passed through alumina columns in a solvent purification system (Innovative Technology).

Reactions were monitored by thin layer chromatography (TLC) using Merck TLC silica gel 60 F254. Compounds were visualized by UV-light at $254 \mathrm{~nm}$ and then with aqueous potassium permanganate stain. 


\section{Preparation and characterization data of substrates 1a-1q}

\section{$\operatorname{Method} A:^{1}$}

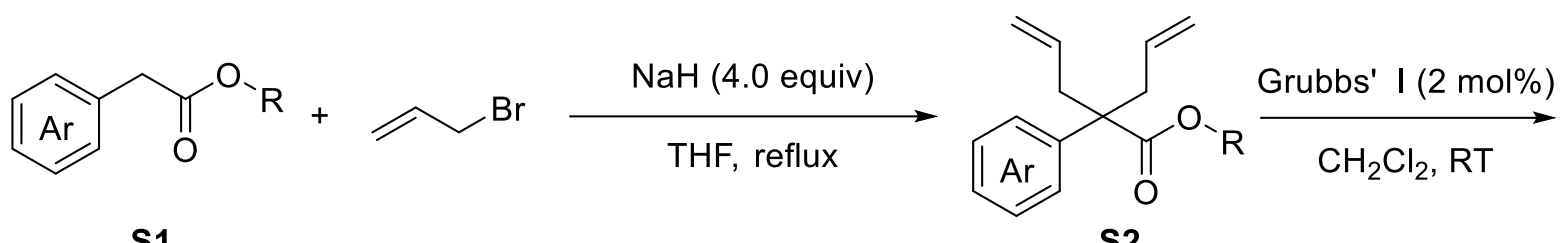

s1

S2

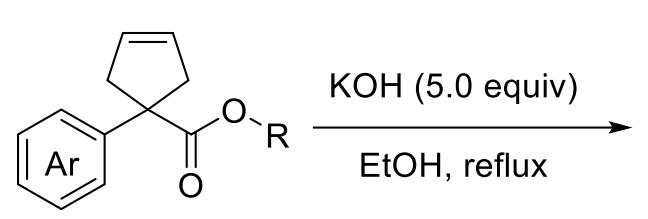

S3

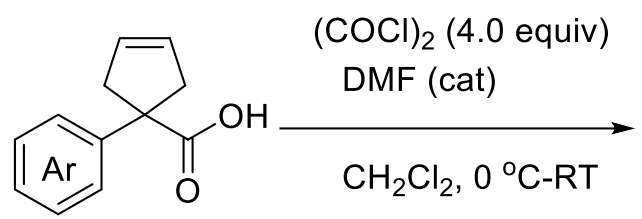

S4

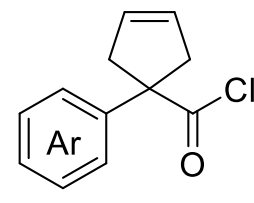

S5
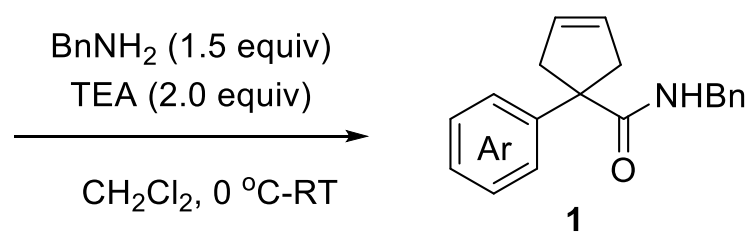

To a suspension of $\mathrm{NaH}(1.6 \mathrm{~g}, 40.0 \mathrm{mmol})$ in THF $(20 \mathrm{~mL})$ at $0{ }^{\circ} \mathrm{C}$ was added a solution of $\mathbf{S 1}$ $(10 \mathrm{mmol})$ in THF $(10 \mathrm{~mL})$ slowly, then the mixture was stirred at $0{ }^{\circ} \mathrm{C}$ for $30 \mathrm{~min}$. Allyl bromide $(4.0 \mathrm{~mL}, 50 \mathrm{mmol})$ was added and the mixture was allowed to reflux until NMR showed that all the $\mathbf{S 1}$ and the monoallylated compound were transferred into $\mathbf{S 2}$. After cooling to $0{ }^{\circ} \mathrm{C}$, saturated $\mathrm{NH}_{4} \mathrm{Cl}$ solution was added slowly to quench the reaction. Then the reaction mixture was extracted with ethyl acetate. The combined organic extracts were washed with brine and dried over $\mathrm{Na}_{2} \mathrm{SO}_{4}$. After concentrating, the residue was used in the next step without further purification.

To a solution of $\mathbf{S 2}(8 \mathrm{mmol})$ in $\mathrm{CH}_{2} \mathrm{Cl}_{2}(40 \mathrm{~mL})$ was added Grubbs' catalyst I $(0.13 \mathrm{~g}, 0.16$ mmol), then the mixture was stirred at RT under $\mathrm{N}_{2}$ until NMR showed the complete conversion. After concentrating, the residue was purified by flash chromatography (silica gel, eluted with petroleum ether/ethyl acetate) to give $\mathbf{S 3}$.

To a solution of $\mathbf{S 3}(8 \mathrm{mmol})$ in ethanol $(30 \mathrm{~mL})$ was added $\mathrm{KOH}(2.3 \mathrm{~g}, 40 \mathrm{mmol})$, then the mixture was refluxed until the complete consumption of $\mathbf{S 3}$ (about $2 \mathrm{hrs}$ ). Ethanol was removed, the residue was dissolved in minimal amount of water, then extracted with $\mathrm{CH}_{2} \mathrm{Cl}_{2}(10 \mathrm{~mL} \times 3)$ and discarded. The aqueous solution was then cooled to $0{ }^{\circ} \mathrm{C}$ and was acidified with $\mathrm{HCl}(3 \mathrm{M})$ carefully. The acidic aqueous solution was extracted with $\mathrm{CH}_{2} \mathrm{Cl}_{2}$. The $\mathrm{CH}_{2} \mathrm{Cl}_{2}$ extracts were dried over $\mathrm{Na}_{2} \mathrm{SO}_{4}$ and concentrated. The residue was used in the next step without further purification. 
To a solution of $\mathbf{S 4}(5 \mathrm{mmol})$ in $\mathrm{CH}_{2} \mathrm{Cl}_{2}(40 \mathrm{~mL})$ at $0{ }^{\circ} \mathrm{C}$ was added $(\mathrm{COCl})_{2}(1.4 \mathrm{~mL}, 16 \mathrm{mmol})$ slowly, followed by 2 drops of DMF. Then the mixture was allowed to stir at $0{ }^{\circ} \mathrm{C}$ for $0.5 \mathrm{~h}$ and at RT for $1 \mathrm{~h}$. Solvents were removed in vacuo and the residue was used in the next step directly. To a solution of $\mathbf{S 5}$ in $\mathrm{CH}_{2} \mathrm{Cl}_{2}(40 \mathrm{~mL})$ at $0{ }^{\circ} \mathrm{C}$ were added slowly benzylamine $(0.8 \mathrm{~mL}, 7.5$ mmol) and triethylamine $(1.7 \mathrm{~mL}, 10 \mathrm{mmol})$ sequentially. After stirring at RT overnight, water was added. The aqueous phase was extracted with $\mathrm{CH}_{2} \mathrm{Cl}_{2}$. The combined organic extracts were washed with diluted $\mathrm{HCl}$ and brine. After drying over $\mathrm{Na}_{2} \mathrm{SO}_{4}$ and concentrating, the residue was purified by flash chromatography (silica gel, eluted with petroleum ether/ethyl acetate) to give $\mathbf{1}$ as solid, which, if necessary can be recrystallized from ethyl acetate.

\section{Method B: ${ }^{1}$}

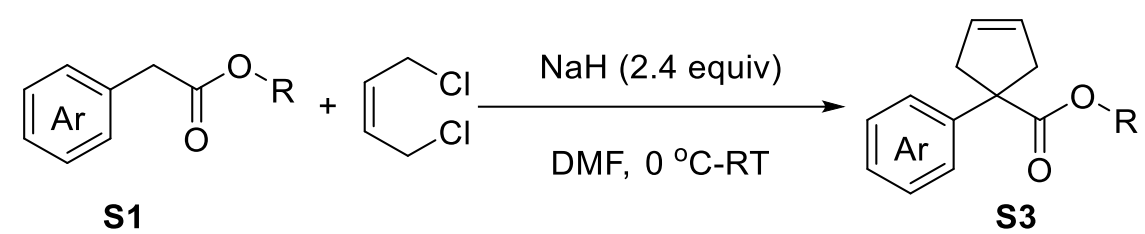

To a suspension of $\mathrm{NaH}(1.9 \mathrm{~g}, 48 \mathrm{mmol})$ in $\mathrm{DMF}(50 \mathrm{~mL})$ at $0{ }^{\circ} \mathrm{C}$ was added a solution of $\mathbf{S 1}$ (20 mmol) in DMF $(20 \mathrm{~mL})$ slowly, then the mixture was stirred at $0{ }^{\circ} \mathrm{C}$ for $2 \mathrm{hrs}$. (Z)-1,4Dichlorobut-2-ene $(2.7 \mathrm{~mL}, 24 \mathrm{mmol})$ in DMF $(10 \mathrm{~mL})$ was added and the mixture was stirred at RT for 2 days. After cooling to $0{ }^{\circ} \mathrm{C}$, saturated $\mathrm{NH}_{4} \mathrm{Cl}$ solution was added slowly to quench the reaction. Then ethyl acetate was added and the mixture was washed with water, brine and dried over $\mathrm{Na}_{2} \mathrm{SO}_{4}$. After concentrating, the residue was used in the next step without further purification.

\section{Method $C:^{1}$}

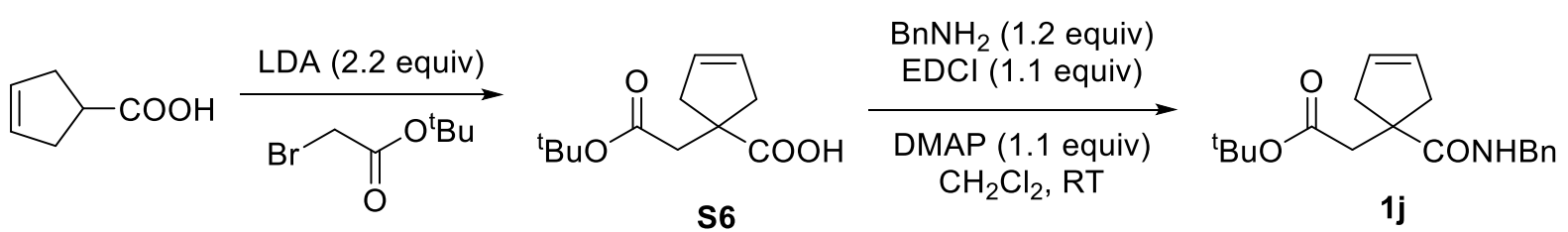

To a solution of cyclopent-3-ene-1-carboxylic acid (2.2 g, $20 \mathrm{mmol})$ in THF (50 mL) was added LDA (prepared from ${ }^{\mathrm{i}} \operatorname{Pr}_{2} \mathrm{NH}(7.1 \mathrm{~mL}, 50 \mathrm{mmol})$ and $\mathrm{BuLi}(18.4 \mathrm{~mL}, 46 \mathrm{mmol})$ prior to use) slowly at $0{ }^{\circ} \mathrm{C}$. Then the mixture was stirred at $0{ }^{\circ} \mathrm{C}$ for $10 \mathrm{~min}$ and at $45{ }^{\circ} \mathrm{C}$ for $2 \mathrm{hrs}$. After cooling to $-78^{\circ} \mathrm{C}$, a solution of tert-butyl bromoacetate $(4.4 \mathrm{~g}, 22 \mathrm{mmol})$ in THF (10 mL) was added slowly. After that, the mixture was stirred at $-78{ }^{\circ} \mathrm{C}$ for $1 \mathrm{~h}$ and then at RT overnight. After cooling to $0{ }^{\circ} \mathrm{C}$, a solution of $1 \mathrm{M} \mathrm{HCl}$ saturated with $\mathrm{NaCl}$ was added, then the reaction mixture was extracted with ether and the ether extracts were dried over $\mathrm{Na}_{2} \mathrm{SO}_{4}$. After 
concentrating, the residue was purified by flash chromatography (silica gel, eluted with petroleum ether/ethyl acetate) to give $\mathbf{S 6}$ as pale yellow solid (2.54 g, 56\%).

To a solution of $\mathbf{S 6}(1.13 \mathrm{~g}, 5 \mathrm{mmol})$ and $\operatorname{DMAP}(0.7 \mathrm{~g}, 5.5 \mathrm{mmol})$ in $\mathrm{CH}_{2} \mathrm{Cl}_{2}(20 \mathrm{~mL})$ at $0{ }^{\circ} \mathrm{C}$ were added benzylamine $(0.7 \mathrm{~mL}, 6 \mathrm{mmol})$ and EDCI $(1.1 \mathrm{~g}, 5.5 \mathrm{mmol})$ sequentially, then the mixture was stirred at RT overnight. $10 \% \mathrm{NaOH}$ solution was added to quench the reaction and the aqueous layer was extracted with $\mathrm{CH}_{2} \mathrm{Cl}_{2}$. The combined dichloromethane extracts were washed with $\mathrm{NaOH}(1 \mathrm{M}), \mathrm{HCl}(1 \mathrm{M})$, saturated $\mathrm{NaHCO}_{3}$ and brine sequentially. After drying over $\mathrm{Na}_{2} \mathrm{SO}_{4}$, the solvents were evaporated and the residue was purified by flash chromatography (silica gel, eluted with petroleum ether/ethyl acetate) to give $\mathbf{1} \mathbf{j}$ as pale yellow solid $(1.0 \mathrm{~g}, 63 \%)$.<smiles>CCCCOC(=O)CC1(C(=O)Nc2ccccc2)CC=CC1</smiles>

$1 \mathrm{j}$

Pale yellow solid, mp $80-81{ }^{\circ} \mathrm{C}$.

${ }^{1} \mathbf{H}$ NMR $\left(400 \mathrm{MHz}, \mathrm{CDCl}_{3}\right) \delta$ 7.34-7.30 (m, 2H), 7.27-7.25 (m, 3H), 6.26 (brs, 1H), 5.70 (s, $2 \mathrm{H}), 4.45(\mathrm{~d}, J=5.6 \mathrm{~Hz}, 2 \mathrm{H}), 2.90-2.84(\mathrm{~m}, 2 \mathrm{H}), 2.69(\mathrm{~s}, 2 \mathrm{H}), 2.55-2.49(\mathrm{~m}, 2 \mathrm{H}), 1.42(\mathrm{~s}, 9 \mathrm{H})$; ${ }^{13}$ C NMR $\left(101 \mathrm{MHz}, \mathrm{CDCl}_{3}\right) \delta 176.7,171.6,138.8,129.5,128.7,127.7,127.4,80.8,50.2,43.9$, 43.81, 43.77, 28.2. IR 3339 (w), 2978 (w), 2928 (w), 2356 (w), 1727 (s), 1644 (s), 1529 (s), 1456 (m), 1430 (w), 1367 (m), 1296 (m), 1245 (m), 1153 (s), 1033 (w), $951(\mathrm{w}), 846(\mathrm{w}), 756$ (w), $733(\mathrm{~m}), 700(\mathrm{~s}), 675(\mathrm{~s})$.

HRMS (ESI/QTOF): Calcd for $\mathrm{C}_{19} \mathrm{H}_{25} \mathrm{NNaO}_{3}{ }^{+}[\mathrm{M}+\mathrm{Na}]^{+} 338.1727$; Found 338.1724.<smiles>O=C(O)Cc1ccccc1[N+](=O)[O-]</smiles><smiles>COCCCCCCOC(=O)OC</smiles>

$\mathrm{MeOH}, 0^{\circ} \mathrm{C}-\mathrm{RT}$<smiles>COC(=O)Cc1ccccc1[N+](=O)[O-]</smiles>

S1<smiles>C=CCC(C(=O)OC)c1ccccc1[N+](=O)[O-]</smiles>

S2-1

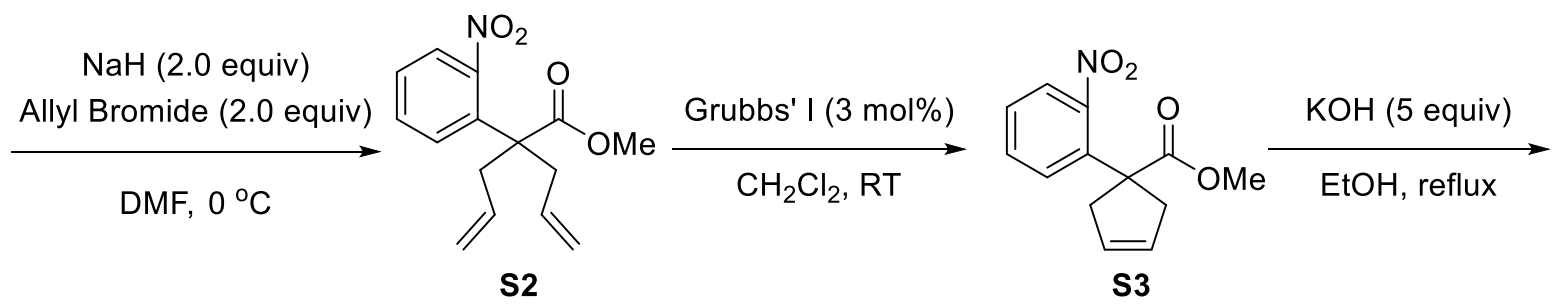




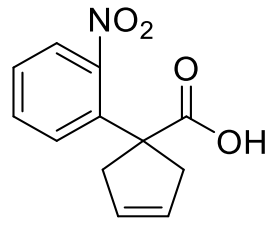

S4

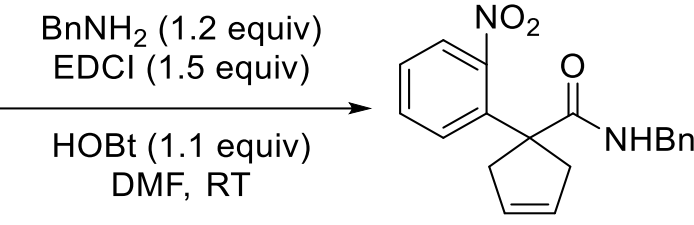

$1 \mathrm{~g}$

To a solution of 2-(2-nitrophenyl)acetic acid $(5.5 \mathrm{~g}, 30 \mathrm{mmol})$ in methanol $(30 \mathrm{~mL})$ at $0{ }^{\circ} \mathrm{C}$ was added $\mathrm{SOCl}_{2}(5 \mathrm{~mL}, 69 \mathrm{mmol})$ slowly, then the mixture was stirred at room temperature overnight. After removing the solvents in vacuo, the residue was dissolved in ethyl acetate and washed with water and brine. After drying over $\mathrm{Na}_{2} \mathrm{SO}_{4}$, the solution was concentrated and the residue was purified by flash chromatography (silica gel, eluted with petroleum ether/ethyl acetate) to give $\mathbf{S 1}$ as pale yellow oil $(5.7 \mathrm{~g}, 98 \%)$.

To a solution of $\mathbf{S 1}(0.98 \mathrm{~g}, 5 \mathrm{mmol})$ and allyl bromide $(0.53 \mathrm{~mL}, 6 \mathrm{mmol})$ in DMF $(10 \mathrm{~mL})$ was added $\mathrm{Cs}_{2} \mathrm{CO}_{3}(2.1 \mathrm{~g}, 6.5 \mathrm{mmol})$, then the suspension was stirred at $60{ }^{\circ} \mathrm{C}$ overnight. After cooling to $0{ }^{\circ} \mathrm{C}$, additional allyl bromide $(0.9 \mathrm{~mL}, 10 \mathrm{mmol})$ was added, followed by $\mathrm{NaH}(0.4$ $\mathrm{g}, 10 \mathrm{mmol})$, then the mixture was allowed to stir at $0{ }^{\circ} \mathrm{C}$ for $4 \mathrm{hrs}$. Water was added slowly and the reaction mixture was extracted with ethyl acetate. The combined organic extracts were washed with water and brine. After drying over $\mathrm{Na}_{2} \mathrm{SO}_{4}$ and concentrating, the residue was purified by flash chromatography (silica gel, eluted with petroleum ether/ethyl acetate) to give $\mathbf{S 2}$ as pale yellow oil $(1.2 \mathrm{~g}, 87 \%){ }^{2}$

$\mathbf{S 3}$ was synthesized from $\mathbf{S 2}$ according to method A.

$\mathbf{S 4}$ was synthesized from $\mathbf{S 3}$ according to method A in $98 \%$ yield for 2 steps. The reaction was run for 36 hrs.

To a solution of S4 (0.38 g, $1.6 \mathrm{mmol})$, EDCI (0.48 g, $2.5 \mathrm{mmol})$ and HOBt (0.25 g, $1.8 \mathrm{mmol})$ in DMF $(5 \mathrm{~mL})$ was added benzylamine $(0.23 \mathrm{~mL}, 2.0 \mathrm{mmol})$, then the mixture was stirred at RT under $\mathrm{N}_{2}$ overnight. Ethyl acetate was added and the solution was washed with water, brine , dried over $\mathrm{Na}_{2} \mathrm{SO}_{4}$. After concentrating, the residue was purified by flash chromatography (silica gel, eluted with petroleum ether/ethyl acetate) to give $\mathbf{1 g}$ as pale yellow solid $(0.52 \mathrm{~g}$, $99 \%)$.

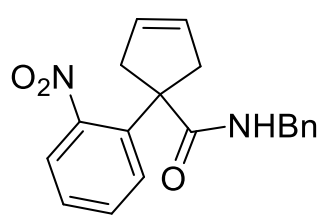

$1 \mathrm{~g}$

Pale yellow solid, mp 91-92 ${ }^{\circ} \mathrm{C}$.

${ }^{1} \mathbf{H}$ NMR $\left(400 \mathrm{MHz}, \mathrm{CDCl}_{3}\right) \delta$ 7.79-7.77 (m, 1H), 7.58-7.50 (m, 2H), 7.42-7.38 (m, 1H), 7.32- 
$7.22(\mathrm{~m}, 5 \mathrm{H}), 5.94$ (brs, 1H), 5.77 (s, 2H), 4.42 (d, $J=5.6 \mathrm{~Hz}, 2 \mathrm{H}), 3.35$ (d, $J=15.3 \mathrm{~Hz}, 2 \mathrm{H})$,

$2.88(\mathrm{~d}, J=15.1 \mathrm{~Hz}, 2 \mathrm{H}) ;{ }^{13} \mathbf{C}$ NMR $\left(101 \mathrm{MHz}, \mathrm{CDCl}_{3}\right) \delta 174.2,149.5,138.6,138.4,132.8$, 130.1, 129.0, 128.8, 128.1, 127.9, 127.6, 125.2, 57.0, 45.0, 44.2. IR 3342 (w), 3317 (w), 2922 (w), 2857 (w), 2163 (w), 1656 (s), 1454 (m), 1357 (s), 1296 (w), 1267 (w), 1244 (m), 1153 (w), $1082(\mathrm{w}), 1032(\mathrm{w}), 951(\mathrm{w}), 910(\mathrm{w}), 852(\mathrm{w}), 850(\mathrm{w}), 785(\mathrm{w}), 723(\mathrm{~s}), 698(\mathrm{~s}), 656(\mathrm{~m})$.

HRMS (ESI/QTOF): Calcd for $\mathrm{C}_{19} \mathrm{H}_{19} \mathrm{~N}_{2} \mathrm{O}_{3}{ }^{+}[\mathrm{M}+\mathrm{H}]^{+} 323.1390$; Found 323.1392.
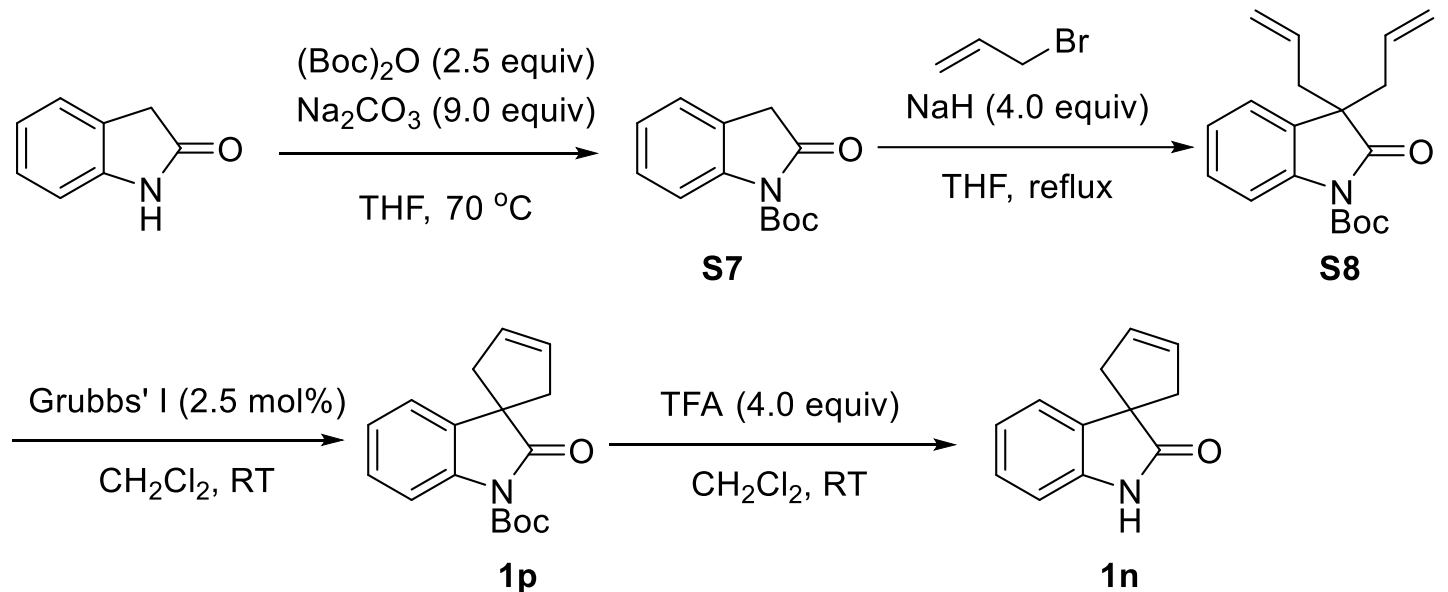

To a solution of indolin-2-one $(4.1 \mathrm{~g}, 30 \mathrm{mmol})$ in THF $(120 \mathrm{~mL})$ were added $\mathrm{Na}_{2} \mathrm{CO}_{3}(28.6 \mathrm{~g}$, $270 \mathrm{mmol})$ and (Boc) $)_{2} \mathrm{O}(16.0 \mathrm{~g}, 75 \mathrm{mmol})$ sequentially, then the suspension was stirred at 70 ${ }^{\circ} \mathrm{C}$ overnight. After cooling to RT, the solid was removed by filtration. The solvents were evaporated and the residue was purified by flash chromatography (silica gel, eluted with petroleum ether/ethyl acetate) to give $\mathbf{S 7}$ as colorless solid (4.1 g, 59\%).

To a suspension of $\mathrm{NaH}(2.8 \mathrm{~g}, 70.0 \mathrm{mmol})$ in THF $(30 \mathrm{~mL})$ at $0{ }^{\circ} \mathrm{C}$ was added a solution of S7 $(4.1 \mathrm{~g}, 17.6 \mathrm{mmol})$ in THF $(10 \mathrm{~mL})$ slowly, then the mixture was stirred at $0{ }^{\circ} \mathrm{C}$ for $1 \mathrm{~h}$. Allyl bromide $(5.6 \mathrm{~mL}, 70 \mathrm{mmol})$ was added and the mixture was allowed to reflux for $5 \mathrm{hrs}$. After cooling to $0{ }^{\circ} \mathrm{C}$, saturated aqueous $\mathrm{NH}_{4} \mathrm{Cl}$ solution was added slowly. Layers were separated and the aqueous layer was extracted with $\mathrm{CH}_{2} \mathrm{Cl}_{2}$, the combined organic extracts were washed with brine and dried over $\mathrm{Na}_{2} \mathrm{SO}_{4}$. After concentrating, the residue was purified by flash chromatography (silica gel, eluted with petroleum ether/ethyl acetate) to give $\mathbf{S 8}$ as colorless oil $(3.1 \mathrm{~g}, 55 \%)$.

To a solution of $\mathbf{S 8}(3.0 \mathrm{~g}, 9.6 \mathrm{mmol})$ in $\mathrm{CH}_{2} \mathrm{Cl}_{2}(60 \mathrm{~mL})$ was added Grubbs' catalyst I $(0.2 \mathrm{~g}$, $0.24 \mathrm{mmol}$ ), then the mixture was stirred at RT under $\mathrm{N}_{2}$ until complete conversion of $\mathbf{S 8}$ (monitored by NMR). After concentrating, the residue was purified by flash chromatography (silica gel, eluted with petroleum ether/ethyl acetate) to give $1 \mathbf{p}^{3 \mathrm{a}}$ as white solid $(2.4 \mathrm{~g}, 87 \%)$. 
<smiles>O=C1Nc2ccccc2C12CC=CC2</smiles>

White solid, mp $78-79^{\circ} \mathrm{C}$.

${ }^{1} \mathbf{H}$ NMR $\left(400 \mathrm{MHz}, \mathrm{CDCl}_{3}\right) \delta$ 7.82-7.80 (m, 1H), 7.29-7.26 (m, 2H), $7.13(\mathrm{t}, J=7.1 \mathrm{~Hz}, 1 \mathrm{H})$, $5.81(\mathrm{~s}, 2 \mathrm{H}), 3.11-3.08(\mathrm{~m}, 2 \mathrm{H}), 2.66-2.62(\mathrm{~m}, 2 \mathrm{H}), 1.65(\mathrm{~s}, 9 \mathrm{H}) ;{ }^{13} \mathbf{C}$ NMR $\left(101 \mathrm{MHz}, \mathrm{CDCl}_{3}\right)$ $\delta 180.4,149.5,138.4,136.2,128.7,128.0,125.1,121.8,114.9,84.4,52.6,46.8,28.3$. IR 2982 (w), 2930 (w), 2359 (w), 1793 (w), 1766 (s), 1729 (s), 1608 (w), 1477 (m), 1369 (w), 1351 (m), 1294 (s), 1253 (m), 1199 (w), 1149 (s), 1082 (w), 1001 (w), 843 (w), 756 (m), 685 (w).

HRMS (ESI/QTOF): Calcd for $\mathrm{C}_{17} \mathrm{H}_{19} \mathrm{NNaO}_{3}{ }^{+}[\mathrm{M}+\mathrm{Na}]^{+}$308.1257; Found 308.1261.

To a solution of $\mathbf{1 p}(0.72 \mathrm{~g}, 2.5 \mathrm{mmol})$ in $\mathrm{CH}_{2} \mathrm{Cl}_{2}(20 \mathrm{~mL})$ was added trifluoroacetic acid (0.8 $\mathrm{mL}, 10 \mathrm{mmol}$ ), then the mixture was stirred at RT for $1 \mathrm{~h}$. After concentrating, the residue was dissolved in $\mathrm{CH}_{2} \mathrm{Cl}_{2}$ and the mixture was neutralized at $0{ }^{\circ} \mathrm{C}$ with saturated $\mathrm{NaHCO}_{3}$ solution. Layers were separated and the aqueous layer was extracted with $\mathrm{CH}_{2} \mathrm{Cl}_{2}$. The combined organic layers were dried over $\mathrm{Na}_{2} \mathrm{SO}_{4}$ and concentrated. The residue was purified by flash chromatography (silica gel, eluted with petroleum ether/ethyl acetate) to give $\mathbf{1 n}$ as colorless solid $(0.45 \mathrm{~g}, 96 \%)$.<smiles>O=C1Nc2ccccc2C12CC=CC2</smiles>

Colorless solid, mp $146-147^{\circ} \mathrm{C}$.

${ }^{1} \mathbf{H}$ NMR $\left(400 \mathrm{MHz}, \mathrm{CDCl}_{3}\right) \delta 8.87(\mathrm{~s}, 1 \mathrm{H}), 7.24(\mathrm{~d}, J=7.4 \mathrm{~Hz}, 1 \mathrm{H}), 7.19(\mathrm{dt}, J=7.7,1.1 \mathrm{~Hz}$, 1H), $7.00(\mathrm{dt}, J=7.6,0.8 \mathrm{~Hz}, 1 \mathrm{H}), 6.92(\mathrm{~d}, J=7.7 \mathrm{~Hz}, 1 \mathrm{H}), 5.85(\mathrm{~s}, 2 \mathrm{H}), 3.07-3.03(\mathrm{~m}, 2 \mathrm{H})$, 2.67-2.64 (m, 2H); ${ }^{13} \mathbf{C}$ NMR (101 MHz, $\left.\mathrm{CDCl}_{3}\right) \delta 184.5,140.0,137.9,129.1,127.8,123.0$, 122.2, 109.8, 52.6, 45.2. IR 3171 (w), 3059 (w), 3032 (w), 2903 (w), 2845 (w), 1702 (s), 1619 (m), 1471 (m), 1336 (m), 1230 (m), 1105 (w), 1053 (w), 951 (w), 802 (w), 750 (s), 687 (m).

HRMS (ESI/QTOF): Calcd for $\mathrm{C}_{12} \mathrm{H}_{12} \mathrm{NO}^{+}[\mathrm{M}+\mathrm{H}]^{+} 186.0913$; Found 186.0918. 

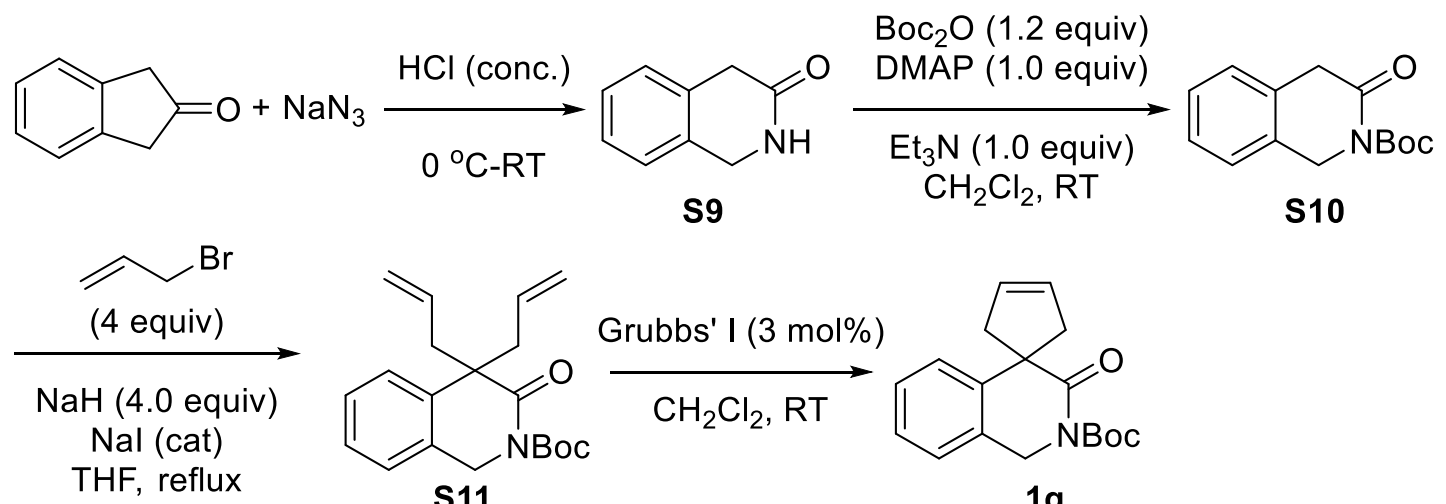

$1 q$

To a solution of 2-indanone $(5.0 \mathrm{~g}, 37.8 \mathrm{mmol})$ in concentrated $\mathrm{HCl}(120 \mathrm{~mL})$ was added $\mathrm{NaN}_{3}$ $(5.0 \mathrm{~g}, 76.9 \mathrm{mmol})$ portionwise at $0{ }^{\circ} \mathrm{C}$. The mixture was stirred at RT overnight. After cooling to $0{ }^{\circ} \mathrm{C}$, dichloromethane was added and the reaction was quenched carefully with solid $\mathrm{K}_{2} \mathrm{CO}_{3}$ and finally adjusted it to $\mathrm{pH}$ 9. Layers were separated and the aqueous layer was extracted with $\mathrm{CH}_{2} \mathrm{Cl}_{2}$. The combined dichloromethane layers were dried over $\mathrm{Na}_{2} \mathrm{SO}_{4}$ and concentrated. The residue was purified by flash chromatography (silica gel, eluted with petroleum ether/ethyl acetate) to give $\mathbf{S 9}$ as pale yellow solid $(3.1 \mathrm{~g}, 56 \%){ }^{4}$

To a solution of $\mathbf{S 9}(1.47 \mathrm{~g}, 10 \mathrm{mmol})$ in $\mathrm{CH}_{2} \mathrm{Cl}_{2}(30 \mathrm{~mL})$ at $\mathrm{RT}$ were added $(\mathrm{Boc})_{2} \mathrm{O}(2.6 \mathrm{~g}, 12$ mmol), DMAP $(1.2 \mathrm{~g}, 10 \mathrm{mmol})$ and $\mathrm{Et}_{3} \mathrm{~N}(1.4 \mathrm{~mL}, 10 \mathrm{mmol})$ sequentially, then the mixture was stirred at RT for $30 \mathrm{hrs}$. After concentrating, the residue was purified by flash chromatography (silica gel, eluted with petroleum ether/ethyl acetate) to give S10 as pale yellow oil $(1.3 \mathrm{~g}, 52 \%)$.

To a suspension of $\mathrm{NaH}(0.85 \mathrm{~g}, 21 \mathrm{mmol})$ in THF $(20 \mathrm{~mL})$ at $0{ }^{\circ} \mathrm{C}$ was added a solution of $\mathbf{S 1 0}$ $(1.3 \mathrm{~g}, 5.2 \mathrm{mmol})$ in THF $(10 \mathrm{~mL})$ slowly, then the mixture was stirred for $1 \mathrm{~h}$ at $0{ }^{\circ} \mathrm{C}$. Allyl bromide $(2.0 \mathrm{~mL}, 21 \mathrm{mmol})$ was added and the mixture was allowed to reflux overnight. After cooling to $0{ }^{\circ} \mathrm{C}$, saturated $\mathrm{NH}_{4} \mathrm{Cl}$ solution was added slowly to quench the reaction. Layers were separated and the aqueous layer was extracted with $\mathrm{CH}_{2} \mathrm{Cl}_{2}$. The combined organic extracts were washed with brine and dried over $\mathrm{Na}_{2} \mathrm{SO}_{4}$. After concentrating, the residue was purified by flash chromatography (silica gel, eluted with petroleum ether/ethyl acetate) to give $\mathbf{S 1 1}$ as colorless oil $(0.89 \mathrm{~g}, 52 \%)$.

To a solution of $\mathbf{S 1 1}(0.89 \mathrm{~g}, 2.7 \mathrm{mmol})$ in $\mathrm{CH}_{2} \mathrm{Cl}_{2}(20 \mathrm{~mL})$ was added Grubbs' catalyst I (0.07 g, $0.085 \mathrm{mmol}$ ), then the mixture was stirred at RT under $\mathrm{N}_{2}$ until NMR shows the complete conversion. After concentrating, the residue was purified by flash chromatography (silica gel, eluted with petroleum ether/ethyl acetate) to give $\mathbf{1 q}$ as brown oil $(0.71 \mathrm{~g}, 87 \%)$. 


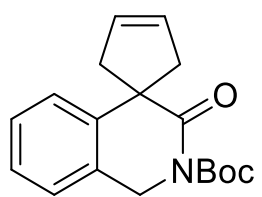

$1 \mathrm{q}$

Brown oil.

${ }^{1} \mathbf{H}$ NMR (400 MHz, $\left.\mathrm{CDCl}_{3}\right)$ 8 7.29-7.24 (m, 3H), 7.21-7.19 (m, 1H), 5.69 (s, 2H), 4.88 (s, 2H), 3.40-3.37 (m, 2H), 2.68-2.64 (m, 2H), 1.57 (s, 9H); ${ }^{13} \mathbf{C ~ N M R ~ ( 1 0 1 ~ M H z , ~ C D C l ~} 3$ ) $\delta ~ 174.1$, 153.0, 141.2, 131.3, 128.2, 127.7, 126.9, 126.2, 124.8, 83.7, 55.5, 47.9, 43.3, 28.2. IR $2980(\mathrm{w})$, 2932 (w), 2163 (w), 1774 (w), 1714 (s), 1459 (w), 1388 (w), 1369 (m), 1301 (s), 1224 (m), 1145 (s), 937 (w), $852(\mathrm{~m}), 756(\mathrm{~m}), 723(\mathrm{~m}), 669(\mathrm{~m})$.

HRMS (ESI/QTOF): Calcd for $\mathrm{C}_{18} \mathrm{H}_{21} \mathrm{NNaO}_{3}{ }^{+}[\mathrm{M}+\mathrm{Na}]^{+}$322.1414; Found 322.1412.<smiles>O=C(NCc1ccccc1)C1(c2ccccc2)CC=CC1</smiles>

1a

White solid, $\mathrm{mp} 87-89^{\circ} \mathrm{C} .{ }^{1 \mathrm{~b}}$

${ }^{1} \mathbf{H}$ NMR (400 MHz, $\left.\mathrm{CDCl}_{3}\right) \delta$ 7.35-7.31 (m, 4H), 7.28-7.22 (m, 4H), 7.11-7.09 (m, 2H), 5.75 (s, 2H), 5.52 (brs, 1H), 4.39 (d, $J=5.8 \mathrm{~Hz}, 2 \mathrm{H}), 3.36-3.29(\mathrm{~m}, 2 \mathrm{H}), 2.88-2.77(\mathrm{~m}, 2 \mathrm{H}) ;{ }^{13} \mathbf{C}$ NMR $\left(101 \mathrm{MHz}, \mathrm{CDCl}_{3}\right) \delta 176.5,145.0,138.6,128.88,128.85,128.7,127.5,127.4,127.1$, 126.8, 58.5, 44.0, 43.9. IR 3340 (w), 3059 (w), 2924 (w), 2853 (w), 2358 (w), 1646 (w), 1525 (w), $1498(w), 1454(w), 1274(w), 1035(w), 949(w), 733(w), 698(w), 673(w)$.

HRMS (ESI/QTOF): Calcd for $\mathrm{C}_{19} \mathrm{H}_{19} \mathrm{NNaO}^{+}[\mathrm{M}+\mathrm{Na}]^{+} 300.1359$; Found 300.1362.

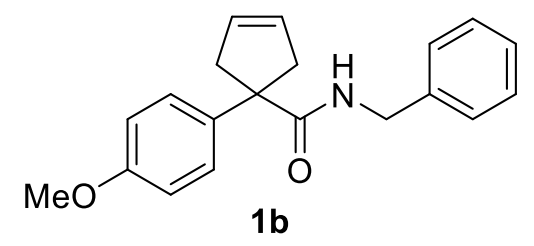

White solid, mp 89-90 ${ }^{\circ} \mathrm{C}$.

${ }^{1} \mathbf{H}$ NMR $\left(400 \mathrm{MHz}, \mathrm{CDCl}_{3}\right) \delta$ 7.32-7.25 (m, 5H), $7.13(\mathrm{~d}, J=6.8 \mathrm{~Hz}, 2 \mathrm{H}), 6.90-6.86(\mathrm{~m}, 2 \mathrm{H})$, $5.76(\mathrm{~s}, 2 \mathrm{H}), 5.59$ (brs, $1 \mathrm{H}), 4.41(\mathrm{~d}, J=6.0 \mathrm{~Hz}, 2 \mathrm{H}), 3.81(\mathrm{~s}, 3 \mathrm{H}), 3.34-3.30(\mathrm{~m}, 2 \mathrm{H}), 2.82-2.79$ $(\mathrm{m}, 2 \mathrm{H}) ;{ }^{13} \mathbf{C ~ N M R}\left(101 \mathrm{MHz}, \mathrm{CDCl}_{3}\right) \delta 176.8,158.6,138.7,137.0,128.9,128.7,128.0,127.5$, 127.4, 114.2, 57.8, 55.4, 44.1, 43.9. IR 3325 (w), 2925 (w), 1647 (m), 1514 (s), 1462 (w), 1242 (s), $1184(\mathrm{~m}), 1042(\mathrm{w}), 824(\mathrm{w}), 697(\mathrm{~s}), 723(\mathrm{~m})$. 
HRMS (nanochip-ESI/LTQ-Orbitrap): Calcd for $\mathrm{C}_{28} \mathrm{H}_{30} \mathrm{NO}_{4}{ }^{+}[\mathrm{M}+\mathrm{H}]^{+}$444.2169; Found 444.2179.<smiles>Cc1cccc(C2(C(=O)NCc3ccccc3)CC=CC2)c1</smiles>

White solid, $\mathrm{mp} 84-86^{\circ} \mathrm{C}$.

${ }^{1} \mathbf{H}$ NMR $\left(400 \mathrm{MHz}, \mathrm{CDCl}_{3}\right) \delta$ 7.28-7.19 (m, 4H), 7.12-7.09 (m, 4H), $7.05(\mathrm{~d}, J=7.4 \mathrm{~Hz}, 1 \mathrm{H})$, $5.74(\mathrm{~s}, 2 \mathrm{H}), 5.54$ (brs, $1 \mathrm{H}), 4.38(\mathrm{~d}, J=5.8 \mathrm{~Hz}, 2 \mathrm{H}), 3.32-3.29(\mathrm{~m}, 2 \mathrm{H}), 2.82-2.78(\mathrm{~m}, 2 \mathrm{H})$, $2.32(\mathrm{~s}, 3 \mathrm{H}) ;{ }^{13} \mathrm{C}$ NMR $\left(101 \mathrm{MHz}, \mathrm{CDCl}_{3}\right) \delta 176.6,145.0,138.7,138.5,128.9,128.70,128.68$, 127.8, 127.7, 127.5, 127.4, 123.8, 58.4, 43.9, 21.7. IR 3319 (w), 2920 (w), 2359 (w), 1646 (s), 1606 (w), 1523 (s), 1454 (m), 1427 (w), 1272 (m), 1215 (w), 1032 (w), 949 (w), 787 (w), 760 (m), $733(\mathrm{~m}), 700(\mathrm{~s})$.

HRMS (ESI/QTOF): Calcd for $\mathrm{C}_{20} \mathrm{H}_{22} \mathrm{NO}^{+}[\mathrm{M}+\mathrm{H}]^{+}$292.1696; Found 292.1702.<smiles>O=C(NCc1ccccc1)C1(c2ccc(F)cc2)CC=CC1</smiles>

White solid, $\mathrm{mp} 88-89^{\circ} \mathrm{C}$.

${ }^{1} \mathbf{H}$ NMR $\left(400 \mathrm{MHz}, \mathrm{CDCl}_{3}\right) \delta$ 7.30-7.22 (m, 5H), 7.12-7.10 (m, 2H), 7.03-6.97 (m, 2H), 5.75 (s, 2H), 5.51 (brs, $1 \mathrm{H}), 4.40(\mathrm{~d}, J=5.7 \mathrm{~Hz}, 2 \mathrm{H}), 3.33-3.28(\mathrm{~m}, 2 \mathrm{H}), 2.79-2.73(\mathrm{~m}, 2 \mathrm{H}) ;{ }^{13} \mathrm{C}$ NMR $\left(101 \mathrm{MHz}, \mathrm{CDCl}_{3}\right) \delta 176.2,161.8(\mathrm{~d}, J=246.0 \mathrm{~Hz}), 140.8(\mathrm{~d}, J=3.3 \mathrm{~Hz}), 138.5,128.9$, 128.8, 128.5 (d, $J=7.9 \mathrm{~Hz}), 127.57,127.55,115.6$ (d, $J=21.3 \mathrm{~Hz}), 58.0,44.1$. IR 3319 (w), 2922 (w), 2363 (w), 1648 (s), 1606 (w), 1509 (s), 1456 (m), 1425 (w), 1292 (m), 1232 (m), $1164(\mathrm{w}), 1018(\mathrm{w}), 951(\mathrm{w}), 837(\mathrm{~m}), 758(\mathrm{~m}), 733(\mathrm{~m}), 700(\mathrm{~m})$.

HRMS (ESI/QTOF): Calcd for $\mathrm{C}_{19} \mathrm{H}_{19} \mathrm{FNO}^{+}[\mathrm{M}+\mathrm{H}]^{+}$296.1445; Found 296.1449.<smiles>O=C(NCc1ccccc1)C1(c2ccc(Cl)cc2)CC=CC1</smiles>

White solid, mp $109-111^{\circ} \mathrm{C}$. 
${ }^{1} \mathbf{H}$ NMR $\left(400 \mathrm{MHz}, \mathrm{CDCl}_{3}\right) \delta$ 7.30-7.23 (m, 7H), $7.11(\mathrm{~d}, J=6.7 \mathrm{~Hz}, 2 \mathrm{H}), 5.74(\mathrm{~s}, 2 \mathrm{H}), 5.53$ (brs, $1 \mathrm{H}), 4.38(\mathrm{~d}, J=5.7 \mathrm{~Hz}, 2 \mathrm{H}), 3.32-3.28(\mathrm{~m}, 2 \mathrm{H}), 2.76-2.73(\mathrm{~m}, 2 \mathrm{H}) ;{ }^{13} \mathbf{C} \mathbf{N M R}(101 \mathrm{MHz}$, $\left.\mathrm{CDCl}_{3}\right) \delta 175.9,143.6,138.4,133.0,128.91,128.89,128.8,128.2,127.59,127.57,58.1,44.1$, 43.9. IR 3327 (w), 3061 (w), 2922 (w), 2359 (w), 1648 (s), 1533 (s), 1494 (s), 1271 (m), 1095 (w), 1016 (m), 1016 (m), $949(\mathrm{w}), 831$ (m), 760 (m), 727 (m).

HRMS (ESI/QTOF): Calcd for $\mathrm{C}_{19} \mathrm{H}_{19} \mathrm{ClNO}^{+}[\mathrm{M}+\mathrm{H}]^{+} 312.1150$; Found 312.1153.

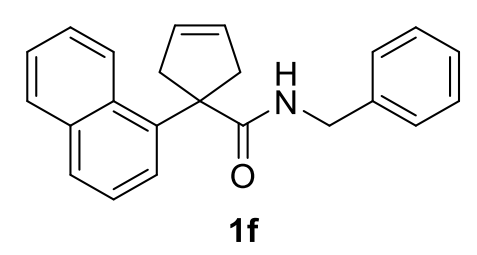

White solid, $\mathrm{mp} 75-77^{\circ} \mathrm{C}$.

${ }^{1} \mathbf{H}$ NMR $\left(400 \mathrm{MHz}, \mathrm{CDCl}_{3}\right) \delta$ 7.96-7.94 (m, 1H), 7.87-7.85 (m, 1H), $7.77(\mathrm{~d}, J=8.2 \mathrm{~Hz}, 1 \mathrm{H})$, 7.52-7.44 (m, 3H), $7.40(\mathrm{t}, J=7.9 \mathrm{~Hz}, 1 \mathrm{H}), 7.14-7.09(\mathrm{~m}, 3 \mathrm{H}), 6.87-6.84(\mathrm{~m}, 2 \mathrm{H}), 5.71(\mathrm{~s}, 2 \mathrm{H})$, $5.37(\mathrm{t}, J=5.0 \mathrm{~Hz}, 1 \mathrm{H}), 4.30(\mathrm{~d}, J=5.8 \mathrm{~Hz}, 2 \mathrm{H}), 3.51(\mathrm{~d}, J=15.5 \mathrm{~Hz}, 2 \mathrm{H}), 2.99$ (d, $J=15.7$ $\mathrm{Hz}, 2 \mathrm{H}) ;{ }^{13} \mathbf{C}$ NMR $\left(101 \mathrm{MHz}, \mathrm{CDCl}_{3}\right) \delta 177.5,139.0,138.4,134.9,131.4,129.3,128.8,128.52$, 128.45, 127.4, 127.3, 126.6, 125.8, 125.4, 125.1, 124.4, 58.1, 44.4, 43.9. IR 3317 (w), 3055 (w), $2926(\mathrm{w}), 2851(\mathrm{w}), 2369(\mathrm{w}), 2356(\mathrm{w}), 2329(\mathrm{w}), 1658(\mathrm{~s}), 1509(\mathrm{~s}), 1456(\mathrm{~m}), 1271(\mathrm{~m})$, $1249(\mathrm{~m}), 1028(\mathrm{w}), 951$ (w), $806(\mathrm{~m}), 781$ (s), 760 (s), 735 (m), 702 (s), $663(\mathrm{~m})$.

HRMS (ESI/QTOF): Calcd for $\mathrm{C}_{23} \mathrm{H}_{22} \mathrm{NO}^{+}[\mathrm{M}+\mathrm{H}]^{+}$328.1696; Found 328.1691.

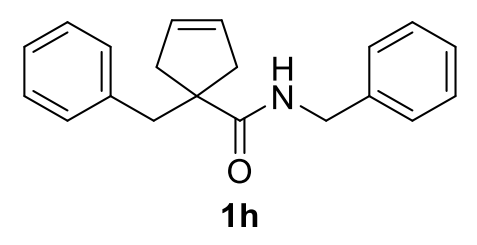

White solid, mp $87-89{ }^{\circ} \mathrm{C}$.

${ }^{1} \mathbf{H}$ NMR $\left(400 \mathrm{MHz}, \mathrm{CDCl}_{3}\right) \delta$ 7.31-7.19 (m, 6H), 7.15-7.10 (m, 4H), 5.70 (s, 2H), 5.48 (brs, $1 \mathrm{H}), 4.37(\mathrm{~d}, J=5.5 \mathrm{~Hz}, 2 \mathrm{H}), 2.98(\mathrm{~s}, 2 \mathrm{H}), 2.68-2.63(\mathrm{~m}, 2 \mathrm{H}), 2.50-2.45(\mathrm{~m}, 2 \mathrm{H}) ;{ }^{13} \mathbf{C} \mathbf{N M R}$ $\left(101 \mathrm{MHz} \mathrm{CDCl}_{3}\right) \delta 176.7,138.29,138.27,130.2,129.1,128.8,128.2,128.1,127.6,126.6$, 54.7, 44.6, 44.0, 41.9. IR 3319 (w), 3059 (w), 3030 (w), 2922 (w), 2847 (w), 1641 (s), 1527 (s), 1498 (m), 1454 (m), 1436 (w), $1286(\mathrm{w}), 1236(\mathrm{~m}), 1032$ (w), 951 (w), 740 (m), 725 (m), 700 (s), 669 (s).

HRMS (ESI/QTOF): Calcd for $\mathrm{C}_{20} \mathrm{H}_{22} \mathrm{NO}^{+}[\mathrm{M}+\mathrm{H}]^{+}$292.1696; Found 292.1699. 
<smiles>CC1(C(=O)NCc2ccccc2)CC=CC1</smiles>

Yellow solid, mp $71-73^{\circ} \mathrm{C} . .^{\text {b }}$

${ }^{1}$ H NMR (400 MHz, $\left.\mathrm{CDCl}_{3}\right)$ d 7.34-7.29 (m, 2H), 7.28-7.26 (m, 3H), 5.85 (brs, 1H), 5.66 (s, 2H), $4.46(\mathrm{~d}, J=5.6 \mathrm{~Hz}, 2 \mathrm{H}), 2.87(\mathrm{~d}, J=14.4 \mathrm{~Hz}, 2 \mathrm{H}), 2.26(\mathrm{~d}, J=14.6 \mathrm{~Hz}, 2 \mathrm{H}), 1.32(\mathrm{~s}, 3 \mathrm{H})$; ${ }^{13}$ C NMR (101 MHz, $\left.\mathrm{CDCl}_{3}\right) \delta 178.4,138.8,129.0,128.8,127.7,127.5,48.3,45.2,43.8,26.4$. IR 3328 (w), 3048 (w), 2923 (w), 2363 (w), 1648 (w), 1532 (w), 1459 (w), 1427 (w), 1294 (w), $1241(\mathrm{w}), 1010(\mathrm{w}), 760(\mathrm{w}), 734(\mathrm{w}), 700(\mathrm{w}), 673(\mathrm{w})$.

HRMS (ESI/QTOF): Calcd for $\mathrm{C}_{14} \mathrm{H}_{18} \mathrm{NO}^{+}[\mathrm{M}+\mathrm{H}]^{+}$216.1383; Found 216.1387.<smiles>O=C(NCc1ccccc1)C1CC=CC1</smiles>

Pale yellow solid, mp $111-112^{\circ} \mathrm{C} .{ }^{1 \mathrm{~b}}$

${ }^{1} \mathbf{H}$ NMR $\left(400 \mathrm{MHz}, \mathrm{CDCl}_{3}\right) \delta$ 7.36-7.32 (m, 2H), 7.28-7.26 (m, 3H), 5.78 (brs, 1H), 5.69 (s, 2H), $4.45(\mathrm{~d}, J=5.6 \mathrm{~Hz}, 2 \mathrm{H}), 3.02-2.94(\mathrm{~m}, 1 \mathrm{H}), 2.69-2.64(\mathrm{~m}, 4 \mathrm{H}) ;{ }^{13} \mathbf{C}$ NMR $(101 \mathrm{MHz}$, $\left.\mathrm{CDCl}_{3}\right) \delta 175.9,138.6,129.4,128.9,127.9,127.6,43.8,43.7,37.1$. IR $3276(\mathrm{w}), 3073(\mathrm{w})$, 2907 (w), 2845 (w), $2371(\mathrm{w}), 2328(\mathrm{w}), 1621(\mathrm{w}), 1565(\mathrm{w}), 1457(\mathrm{w}), 1387(\mathrm{w}), 1350(\mathrm{w})$, $1299(\mathrm{w}), 1241(\mathrm{w}), 1180(\mathrm{w}), 1039(\mathrm{w}), 1073(\mathrm{w}), 749(\mathrm{w}), 691(\mathrm{w})$.

HRMS (ESI/QTOF): Calcd for $\mathrm{C}_{13} \mathrm{H}_{16} \mathrm{NO}^{+}[\mathrm{M}+\mathrm{H}]^{+}$202.1226; Found 202.1229.<smiles>CC(C)CNC(=O)C1(c2ccccc2)CC=CC1</smiles>

Pale yellow solid, $\mathrm{mp} 55-58{ }^{\circ} \mathrm{C}$.

${ }^{1}$ H NMR $\left(400 \mathrm{MHz}, \mathrm{CDCl}_{3}\right) \delta$ 7.35-7.30 (m, 4H), 7.26-7.21 (m, 1H), $5.72(\mathrm{~s}, 2 \mathrm{H}), 5.37$ (brs, $1 \mathrm{H}), 3.30(\mathrm{~d}, J=15.2 \mathrm{~Hz}, 2 \mathrm{H}), 2.99(\mathrm{t}, J=6.4 \mathrm{~Hz}, 2 \mathrm{H}), 2.80(\mathrm{~d}, J=15.2 \mathrm{~Hz}, 2 \mathrm{H}), 1.71-1.61$ $(\mathrm{m}, 1 \mathrm{H}), 0.76(\mathrm{~d}, J=6.8 \mathrm{~Hz}, 6 \mathrm{H}) ;{ }^{13} \mathbf{C} \mathbf{N M R}\left(101 \mathrm{MHz}, \mathrm{CDCl}_{3}\right) \delta 176.3,145.2,128.7,128.6$, 126.8, 126.7, 58.4, 47.1, 43.8, 28.4, 19.9. IR 3339 (w), 2959 (w), 2926 (w), 2365 (w), 2161 (w), 1644 (s), 1525 (s), 1498 (m), 1272 (m), 1159 (w), 1039 (w), 951 (w), 760 (w), 735 (w), $700(\mathrm{~s}), 673(\mathrm{~m})$. 
HRMS (ESI/QTOF): Calcd for $\mathrm{C}_{16} \mathrm{H}_{22} \mathrm{NO}^{+}[\mathrm{M}+\mathrm{H}]^{+}$244.1696; Found 244.1703.

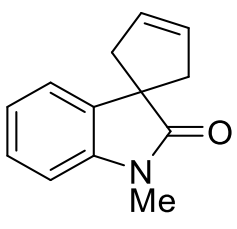

10

Colorless solid, mp $92-94{ }^{\circ} \mathrm{C} .{ }^{3}$

${ }^{1} \mathbf{H}$ NMR $\left(400 \mathrm{MHz}, \mathrm{CDCl}_{3}\right) \delta$ 7.27-7.23 (m, 2H), 7.04-7.00 (m, 1H), 6.83-6.81 (m, 1H), 5.84 (s, 2H), $3.23(\mathrm{~s}, 3 \mathrm{H}), 3.05-2.98(\mathrm{~m}, 2 \mathrm{H}), 2.62-2.56(\mathrm{~m}, 2 \mathrm{H}) ;{ }^{13} \mathbf{C} \mathbf{N M R}\left(101 \mathrm{MHz}, \mathrm{CDCl}_{3}\right) \delta$ 181.6, 142.7, 137.6, 129.1, 127.8, 123.0, 121.7, 107.9, 52.2, 45.1, 26.5. IR 2918 (w), 2897 (w), 2839 (w), 1704 (s), 1612 (m), 1494 (m), 1471 (m), 1421 (w), 1378 (m), 1346 (m), 1313 (w), 1267 (m), 1242 (w), 1188 (w), 1116 (w), 1085 (m), 1030 (m), 985 (w), $970(\mathrm{w}), 952$ (w), 926 $(\mathrm{w}), 875(\mathrm{w}), 812(\mathrm{w}), 750(\mathrm{~s}), 688(\mathrm{~s})$.

HRMS (ESI/QTOF): Calcd for $\mathrm{C}_{13} \mathrm{H}_{14} \mathrm{NO}^{+}[\mathrm{M}+\mathrm{H}]^{+}$200.1070; Found 200.1072. 
3. General procedure for catalytic asymmetric oxidative Heck reaction and characterization data of products 3aa-3qg

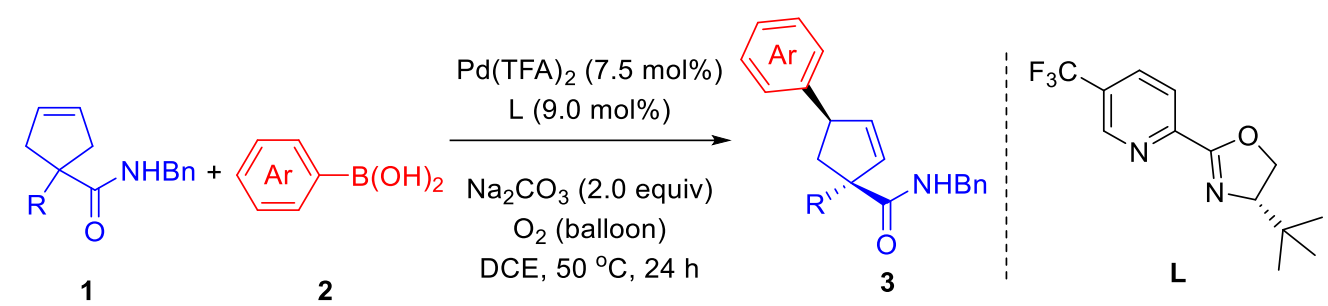

A $10 \mathrm{~mL}$ reaction tube was charged with $\mathrm{Pd}(\mathrm{TFA})_{2}(2.5 \mathrm{mg}, 0.0075 \mathrm{mmol})$, ligand $(2.5 \mathrm{mg}$, $0.009 \mathrm{mmol})$ and DCE $(1.0 \mathrm{~mL})$, then the mixture was stirred at RT for $30 \mathrm{~min} .1(0.1 \mathrm{mmol})$, 2 (0.2 mmol), $\mathrm{Na}_{2} \mathrm{CO}_{3}(21.2 \mathrm{mg}, 0.2 \mathrm{mmol})$ and additional DCE $(1 \mathrm{~mL})$ were added sequentially. After purging with $\mathrm{O}_{2}$, the mixture was stirred on an oil bath at $50{ }^{\circ} \mathrm{C}$ with an $\mathrm{O}_{2}$ balloon for 24 hrs. After cooling to RT, the mixture was filtered through a short pad of celite with acetone washing. The solution was concentrated and subjected to ${ }^{1} \mathrm{H}$ NMR for $\mathrm{dr}$ value analysis. The residue was purified by flash chromatography (silica gel, eluted with petroleum ether/ethyl acetate) to give product 3 .

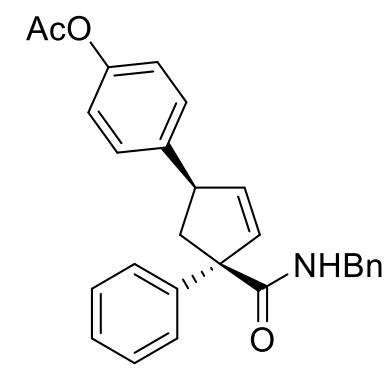

3 aa

According to the general procedure, 3aa was obtained in $89 \%$ yield $(36.6 \mathrm{mg})$ as colorless oil with >19:1 dr and 93\% ee which was measured by SFC on Chiralcel OJ-H $(15 \% \mathrm{MeOH}$ in supercritical $\mathrm{CO}_{2}$ as eluent; flow rate $4.0 \mathrm{~mL} / \mathrm{min}$; $\mathrm{tR}$ (major) $=13.2 \mathrm{~min}$., $\mathrm{tR}$ (minor) $=6.1 \mathrm{~min}$.). $[\alpha]_{D}^{25}+18.1 .\left(c 0.91, \mathrm{CHCl}_{3}\right)$.

${ }^{1} \mathbf{H}$ NMR (400 MHz, $\left.\mathrm{CDCl}_{3}\right) \delta$ 7.29-7.25 (m, 4H), 7.20-7.17 (m, 6H), 7.06 (d, J = 7.6 Hz, 2H), $6.93(\mathrm{~d}, J=7.6 \mathrm{~Hz}, 2 \mathrm{H}), 6.12-6.10(\mathrm{~m}, 1 \mathrm{H}), 5.96-5.95(\mathrm{~m}, 1 \mathrm{H}), 5.57$ (brs, 1H), 4.38 (dd, $J=$ 14.8, $6.3 \mathrm{~Hz}, 1 \mathrm{H}), 4.32(\mathrm{dd}, J=14.8,6.2 \mathrm{~Hz}, 1 \mathrm{H}), 3.86(\mathrm{t}, J=7.8 \mathrm{~Hz}, 1 \mathrm{H}), 2.82(\mathrm{dd}, J=13.2$, $8.0 \mathrm{~Hz}, 1 \mathrm{H}), 2.62(\mathrm{dd}, J=13.2,8.0 \mathrm{~Hz}, 1 \mathrm{H}), 2.22(\mathrm{~s}, 3 \mathrm{H}) ;{ }^{13} \mathbf{C ~ N M R}\left(101 \mathrm{MHz}, \mathrm{CDCl}_{3}\right) \delta 174.9$, $169.8,149.3,143.6,141.9,138.4,137.6,134.1,129.0,128.8,128.7,127.6,127.5,127.4,126.9$, 121.7, 65.6, 50.0, 45.9, 44.0, 21.3. IR 2942 (w), 2940 (w), 2930 (w), 2889 (w), 2365 (m), 1758 (s), 1652 (s), 1509 (s), 1508 (s), 1454 (m), 1369 (m), 1168 (m), 1018 (m), 991 (w), 931 (w), 912 (m), 858 (w), 833 (m), 814 (w), 793 (m), 769 (m), 754 (w), 752 (m), 733 (m), 731 (m), 696 
(s), $677(\mathrm{~s})$.

HRMS (nanochip-ESI/LTQ-Orbitrap): Calcd for $\mathrm{C}_{27} \mathrm{H}_{25} \mathrm{NNaO}_{3}{ }^{+}[\mathrm{M}+\mathrm{Na}]^{+}$434.1727; Found 434.1718 .

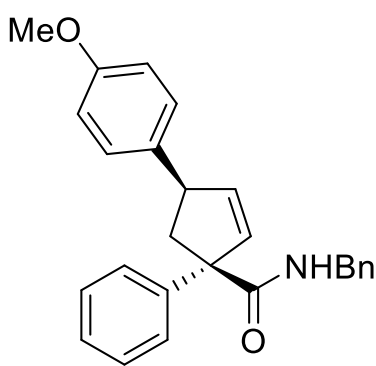

$3 a b$

According to the general procedure, 3ab was obtained in $89 \%$ yield (34.2 $\mathrm{mg}$ ) as colorless oil with $>19: 1 \mathrm{dr}$ and $91 \%$ ee which was measured by SFC on Chiralcel $\mathrm{OJ}-\mathrm{H}(8 \% \mathrm{MeOH}$ in supercritical $\mathrm{CO}_{2}$ as eluent; flow rate $4.0 \mathrm{~mL} / \mathrm{min}$; $\mathrm{tR}$ (major) $=21.3 \mathrm{~min}$., $\mathrm{tR}$ (minor) $=17.3$ $\min .) .[\alpha]_{D}^{25}+63.5 .\left(c 0.77, \mathrm{CHCl}_{3}\right)$.

${ }^{1} \mathbf{H}$ NMR $\left(400 \mathrm{MHz}, \mathrm{CDCl}_{3}\right) \delta$ 7.36-7.32 (m, 4H), 7.27-7.23 (m, 4H), 7.17-7.13 (m, 4H), 6.83 (d, $J=8.0 \mathrm{~Hz}, 2 \mathrm{H}), 6.17-6.16$ (m, 1H), 6.02-6.01 (m, 1H), 5.66 (brs, 1H), 4.45 (dd, $J=14.9$, $5.8 \mathrm{~Hz}, 1 \mathrm{H}), 4.39$ (dd, $J=14.8,5.5 \mathrm{~Hz}, 1 \mathrm{H}), 3.89(\mathrm{t}, J=8.0 \mathrm{~Hz}, 1 \mathrm{H}), 3.78$ (s, 3H), 2.85 (dd, $J$ $=12.0,8.0 \mathrm{~Hz}, 1 \mathrm{H}), 2.69(\mathrm{dd}, J=12.0,8.0 \mathrm{~Hz}, 1 \mathrm{H}) ;{ }^{13} \mathbf{C ~ N M R}\left(101 \mathrm{MHz}, \mathrm{CDCl}_{3}\right) \delta 175.0$, $158.4,143.8,138.5,138.2$, 136.4, 133.7, 128.9, 128.7, 128.6, 127.6, 127.5, 127.3 , 126.8, 114.1, 65.5, 55.4, 49.8, 46.1, 44.0. IR 3339 (w), 3331 (w), 3329 (w), 3057 (w), 3028 (w), 2930 (w), 2365 (w), 1652 (m), 1610 (w), $1513(\mathrm{~s}), 1452$ (w), 1357 (w), $1301(\mathrm{w}), 1249(\mathrm{~s}), 1180(\mathrm{w}), 1107$ (w), $1078(\mathrm{w}), 1035(\mathrm{w}), 833(\mathrm{w}), 796(\mathrm{w}), 760(\mathrm{w}), 735$ (m), $721(\mathrm{w}), 702(\mathrm{~m}), 671(\mathrm{w})$.

HRMS (ESI/QTOF): Calcd for $\mathrm{C}_{26} \mathrm{H}_{26} \mathrm{NO}_{2}{ }^{+}[\mathrm{M}+\mathrm{H}]^{+}$384.1958; Found 384.1960.

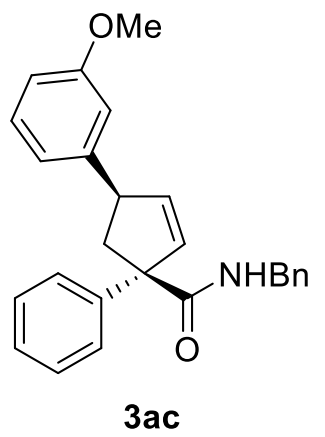

According to the general procedure, 3ac was obtained in $61 \%$ yield $(23.4 \mathrm{mg})$ as colorless oil with $>19: 1 \mathrm{dr}$ and $95 \%$ ee which was measured by SFC on Chiralcel IA (10\% $\mathrm{MeOH}$ in supercritical $\mathrm{CO}_{2}$ as eluent; flow rate $4.0 \mathrm{~mL} / \mathrm{min}$; $\mathrm{tR}$ (major) $=13.6 \mathrm{~min}$., $\mathrm{tR}$ (minor) $=11.2$ 
min.). $[\alpha]_{D}^{25}+30.5\left(c 1.11, \mathrm{CHCl}_{3}\right)$.

${ }^{1} \mathbf{H}$ NMR $\left(400 \mathrm{MHz}, \mathrm{CDCl}_{3}\right) \delta$ 7.31-7.26 (m, 4H), 7.21-7.12 (m, 5H), $7.06(\mathrm{~d}, J=7.2 \mathrm{~Hz}, 2 \mathrm{H})$, $6.76(\mathrm{~d}, J=7.6 \mathrm{~Hz}, 1 \mathrm{H}), 6.73(\mathrm{~s}, 1 \mathrm{H}), 6.69(\mathrm{~d}, J=8.4 \mathrm{~Hz}, 1 \mathrm{H}), 6.12-6.11(\mathrm{~m}, 1 \mathrm{H}), 5.98(\mathrm{~d}, J=$ $5.2 \mathrm{~Hz}, 1 \mathrm{H}), 5.59$ (brs, 1H), 4.37 (dd, $J=15.0,5.7 \mathrm{~Hz}, 1 \mathrm{H}), 4.31$ (dd, $J=14.9,5.6 \mathrm{~Hz}, 1 \mathrm{H})$, $3.83(\mathrm{t}, J=7.8 \mathrm{~Hz}, 1 \mathrm{H}), 3.71(\mathrm{~s}, 3 \mathrm{H}), 2.82(\mathrm{dd}, J=13.2,8.0 \mathrm{~Hz}, 1 \mathrm{H}), 2.64(\mathrm{dd}, J=13.2,8.0$ $\mathrm{Hz}, 1 \mathrm{H}) ;{ }^{13} \mathbf{C}$ NMR $\left(101 \mathrm{MHz}, \mathrm{CDCl}_{3}\right) \delta 174.9,159.9,145.9,143.6,138.4,137.7,134.1,129.6$, $128.9,128.7,127.6,127.5,127.3,126.8,120.0,113.2$, 112.1, 65.5, 55.3, 50.5, 45.8, 44.0. IR $3383(w), 3364(w), 3362$ (w), 3333 (w), 3323 (w), 3308 (w), 3292 (w), $3061(w), 3032(w)$, 2934 (w), 2932 (w), 2834 (w), 2022 (w), 1652 (s), 1602 (m), 1585 (m), 1529 (s), 1494 (s), 1454 (m), 1434 (m), 1326 (m), 1324 (m), 1269 (s), 1161 (m), 1043 (m), 870 (w), 804 (m), 779 (s), $762(\mathrm{~s}), 746(\mathrm{~m}), 733(\mathrm{~m}), 698(\mathrm{~s}), 673(\mathrm{~s})$.

HRMS (ESI/QTOF): Calcd for $\mathrm{C}_{26} \mathrm{H}_{26} \mathrm{NO}_{2}{ }^{+}[\mathrm{M}+\mathrm{H}]^{+}$384.1958; Found 384.1960.

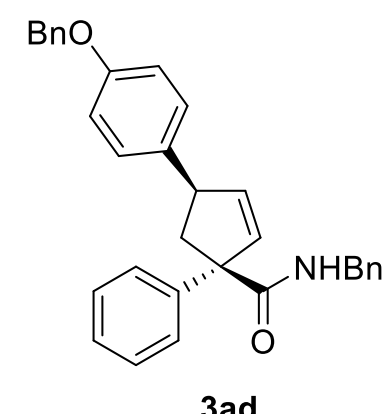

According to the general procedure, 3ad was obtained in $76 \%$ yield (35.0 mg) as colorless oil with >19:1 dr and $89 \%$ ee which was measured by SFC on Chiralcel IA $(20 \% \mathrm{MeOH}$ in supercritical $\mathrm{CO}_{2}$ as eluent; flow rate $4.0 \mathrm{~mL} / \mathrm{min}$; $\mathrm{tR}$ (major) $=16.3 \mathrm{~min} ., \mathrm{tR}$ (minor) $=15.1$ min.). $[\alpha]_{D}^{25}+61.6$. ( $\left.c 0.41, \mathrm{CHCl}_{3}\right)$.

${ }^{1} \mathbf{H}$ NMR $\left(400 \mathrm{MHz}, \mathrm{CDCl}_{3}\right) \delta$ 7.44-7.32 (m, 9H), 7.30-7.23 (m, 4H), 7.17-7.13 (m, 4H), 6.91 $(\mathrm{d}, J=8.0 \mathrm{~Hz}, 2 \mathrm{H}), 6.16(\mathrm{dd}, J=5.2,2.0 \mathrm{~Hz}, 1 \mathrm{H}), 6.02(\mathrm{dd}, J=5.5,1.8 \mathrm{~Hz}, 1 \mathrm{H}), 5.63(\mathrm{t}, J=$ $8.0 \mathrm{~Hz}, 1 \mathrm{H}), 5.05$ (s, 2H), $4.45(\mathrm{dd}, J=14.9,5.8 \mathrm{~Hz}, 1 \mathrm{H}), 4.40(\mathrm{dd}, J=14.9,5.7 \mathrm{~Hz}, 1 \mathrm{H}), 3.89$ $(\mathrm{tt}, J=7.9,3.0 \mathrm{~Hz}, 1 \mathrm{H}), 2.85(\mathrm{dd}, J=13.2,8.0 \mathrm{~Hz}, 1 \mathrm{H}), 2.70(\mathrm{dd}, J=13.2,8.0 \mathrm{~Hz}, 1 \mathrm{H}) ;{ }^{13} \mathrm{C}$ NMR (101 MHz, $\left.\mathrm{CDCl}_{3}\right) \delta 175.0,157.6,143.8,138.5,138.2,137.3,136.7,133.7,128.9,128.8$, 128.72, 128.68, 128.1, 127.6, 127.5, 127.3, 126.9, 115.0, 70.2, 65.5, 49.8, 46.1, 44.0. IR 3366 (w), $3327(\mathrm{w}), 3308(\mathrm{w}), 3061(\mathrm{w}), 3030(\mathrm{w}), 1652(\mathrm{~m}), 1610(\mathrm{w}), 1511(\mathrm{~s}), 1452(\mathrm{w}), 1361$ (w), 1240 (m), 1178 (w), $1074(\mathrm{w}), 1022$ (m), 904 (w), 831 (m), $806(\mathrm{w}), 760$ (s), 742 (m), 727 (m), $696(\mathrm{~s}), 677(\mathrm{~m})$.

HRMS (ESI/QTOF): Calcd for $\mathrm{C}_{32} \mathrm{H}_{30} \mathrm{NO}_{2}{ }^{+}[\mathrm{M}+\mathrm{H}]^{+}$460.2271; Found 460.2276. 


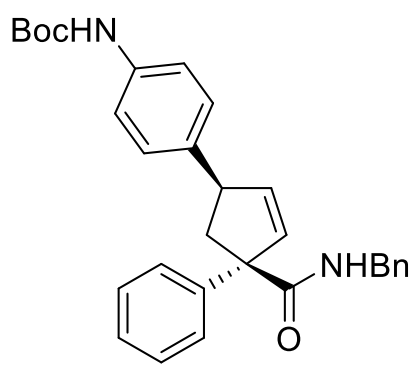

3ae

According to the general procedure, 3ae was obtained in $79 \%$ yield $(37.0 \mathrm{mg}$ ) as white solid (mp $147-150{ }^{\circ} \mathrm{C}$ ) with 5:1 dr and $88 \%$ ee which was measured by SFC on Chiralcel OJ-H (15\% $\mathrm{MeOH}$ in supercritical $\mathrm{CO}_{2}$ as eluent; flow rate $4.0 \mathrm{~mL} / \mathrm{min}$; $\mathrm{tR}$ (major) $=9.5 \mathrm{~min}$., $\mathrm{tR}$ (minor) $=7.5 \min .) .[\alpha]_{D}^{25}-158.9 .\left(c 1.35, \mathrm{CHCl}_{3}\right)$.

${ }^{1}$ H NMR $\left(400 \mathrm{MHz}, \mathrm{CDCl}_{3}\right) \delta$ 7.36-7.32 (m, 4H), 7.27-7.25 (m, 6H), 7.20-7.11 (m, 4H), 6.50 (brs, 1H), 6.18-6.17 (m, 1H), 6.02-6.00 (m, 1H), 5.66 (brs, 1H), 4.46-4.40 (m, 2H), 3.88 (t, $J=$ $8.0 \mathrm{~Hz}, 1 \mathrm{H}), 2.83(\mathrm{dd}, J=12.0,8.0 \mathrm{~Hz}, 1 \mathrm{H}), 2.69(\mathrm{dd}, J=12.0,8.0 \mathrm{~Hz}, 1 \mathrm{H}), 1.51(\mathrm{~s}, 9 \mathrm{H}) ;{ }^{13} \mathrm{C}$ NMR $\left(101 \mathrm{MHz}, \mathrm{CDCl}_{3}\right) \delta 175.0,153.0,143.6,138.9,138.4,137.9,136.9,133.9,128.9,128.8$, 128.2, 127.6, 127.5, 127.3, 126.8, 119.0, 80.5, 65.4, 49.9, 45.9, 44.0, 28.5. IR $3348(w), 3325$ (w), 3312 (w), $3310(\mathrm{w}), 3296(\mathrm{w}), 3057$ (w), $2361(\mathrm{w}), 2331(\mathrm{w}), 2159(\mathrm{w}), 2022(\mathrm{w}), 1801$ (w), 1702 (m), 1652 (m), 1598 (w), 1573 (w), 1523 (s), 1454 (w), 1415 (w), 1367 (w), 1317 (w), 1244 (m), 1163 (s), 1055 (w), 1014 (w), 870 (w), 837 (w), 796 (w), 771 (w), 754 (w), 723 (m), $700(\mathrm{w}), 681(\mathrm{w}), 661(\mathrm{w})$.

HRMS (ESI/QTOF): Calcd for $\mathrm{C}_{30} \mathrm{H}_{32} \mathrm{~N}_{2} \mathrm{NaO}_{3}{ }^{+}[\mathrm{M}+\mathrm{Na}]^{+} 491.2305$; Found 491.2307.

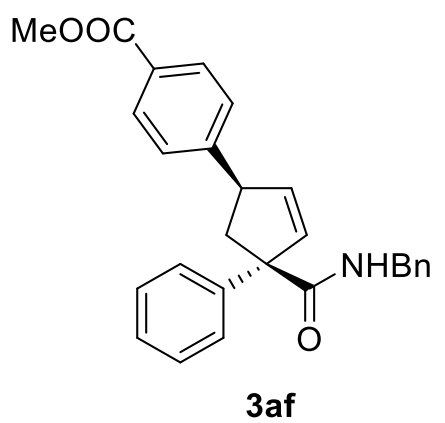

According to the general procedure, 3af was obtained in $75 \%$ yield (30.9 mg) as colorless oil with >19:1 dr and 96\% ee which was measured by SFC on Chiralcel $\mathrm{OJ}-\mathrm{H}(8 \% \mathrm{MeOH}$ in supercritical $\mathrm{CO}_{2}$ as eluent; flow rate $4.0 \mathrm{~mL} / \mathrm{min}$; $\mathrm{tR}$ (major) $=16.7 \mathrm{~min} ., \mathrm{tR}$ (minor) $=21.3$ $\min .) .[\alpha]_{D}^{25}+57.4\left(c 0.73, \mathrm{CHCl}_{3}\right)$.

${ }^{1} \mathbf{H}$ NMR $\left(400 \mathrm{MHz}, \mathrm{CDCl}_{3}\right) \delta 7.89(\mathrm{~d}, J=8.4 \mathrm{~Hz}, 2 \mathrm{H}), 7.29-7.28(\mathrm{~m}, 4 \mathrm{H}), 7.25-7.16(\mathrm{~m}, 6 \mathrm{H})$, 7.07-7.05 (m, 2H), $6.15(\mathrm{dd}, J=5.6,2.4 \mathrm{~Hz}, 1 \mathrm{H}), 5.97(\mathrm{dd}, J=5.6,2.0 \mathrm{~Hz}, 1 \mathrm{H}), 5.59(\mathrm{t}, J=$ 
$5.8 \mathrm{~Hz}, 1 \mathrm{H}), 4.37(\mathrm{dd}, J=14.9,5.9 \mathrm{~Hz}, 1 \mathrm{H}), 4.31(\mathrm{dd}, J=14.9,5.8 \mathrm{~Hz}, 1 \mathrm{H}), 3.91(\mathrm{tt}, J=8.0$, $2.3 \mathrm{~Hz}, 1 \mathrm{H}), 3.82(\mathrm{~s}, 3 \mathrm{H}), 2.87(\mathrm{dd}, J=13.2,7.9 \mathrm{~Hz}, 1 \mathrm{H}), 2.63(\mathrm{dd}, J=13.2,8.0 \mathrm{~Hz}, 1 \mathrm{H}) ;{ }^{13} \mathrm{C}$ NMR $\left(101 \mathrm{MHz}, \mathrm{CDCl}_{3}\right) \delta 174.7,167.2,149.7,143.5,138.3,137.1,134.6,130.0,129.0,128.8$, 128.5, 127.7, 127.59, 127.55, 127.47, 126.8, 65.6, 52.2, 50.5, 45.6, 44.0. IR 3356 (w), 3339 (w), 3337 (w), 3335 (w), 3333 (w), 3331 (w), 2947 (w), 2359 (w), 2157 (w), 1720 (s), 1654 (m), 1610 (w), 1521 (m), 1519 (m), 1498 (w), 1438 (w), 1436 (w), 1313 (w), 1282 (s), 1182 (w), $1112(w), 1020(w), 976(w), 968(w), 939(w), 937(w), 914(w), 891(w), 852(w), 816$ (w), $789(\mathrm{w}), 773(\mathrm{~m}), 754(\mathrm{w}), 725(\mathrm{~m}), 696(\mathrm{~m}), 665(\mathrm{w}), 656(\mathrm{w})$.

HRMS (ESI/QTOF): Calcd for $\mathrm{C}_{27} \mathrm{H}_{26} \mathrm{NO}_{3}{ }^{+}[\mathrm{M}+\mathrm{H}]^{+}$412.1907; Found 412.1910.

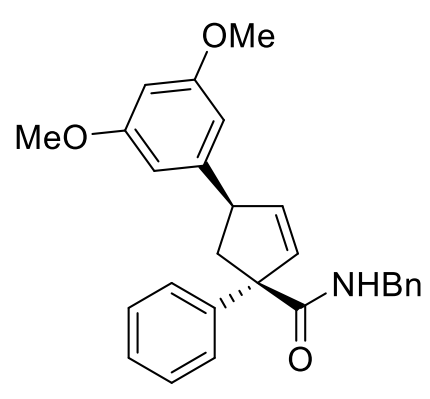

3ag

According to the general procedure, 3ag was obtained in $71 \%$ yield $(29.2 \mathrm{mg})$ as colorless oil with 18:1 dr and $97 \%$ ee which was measured by SFC on Chiralcel $\mathrm{OJ}-\mathrm{H}(10 \% \mathrm{MeOH}$ in supercritical $\mathrm{CO}_{2}$ as eluent; flow rate $4.0 \mathrm{~mL} / \mathrm{min}$; $\mathrm{tR}$ (major) $=8.1 \mathrm{~min} ., \mathrm{tR}$ (minor) $=10.3$ $\min$.). $[\alpha]_{D}^{25}+62.2\left(c 1.32, \mathrm{CHCl}_{3}\right)$.

${ }^{1} \mathbf{H}$ NMR (400 MHz, $\left.\mathrm{CDCl}_{3}\right) \delta$ 7.38-7.32 (m, 4H), 7.28-7.22 (m, 4H), 7.14-7.12 (m, 2H), 6.41 $(\mathrm{d}, J=2.3 \mathrm{~Hz}, 2 \mathrm{H}), 6.32(\mathrm{t}, J=2.3 \mathrm{~Hz}, 1 \mathrm{H}), 6.20(\mathrm{dd}, J=5.6,2.5 \mathrm{~Hz}, 1 \mathrm{H}), 6.05(\mathrm{dd}, J=5.6$, $1.9 \mathrm{~Hz}, 1 \mathrm{H}), 5.66(\mathrm{t}, J=5.8 \mathrm{~Hz}, 1 \mathrm{H}), 4.44(\mathrm{dd}, J=15.0,5.8 \mathrm{~Hz}, 1 \mathrm{H}), 4.31(\mathrm{dd}, J=15.0,5.8 \mathrm{~Hz}$, $1 \mathrm{H}), 3.87$ (tt, $J=8.0,2.3 \mathrm{~Hz}, 1 \mathrm{H}), 3.76(\mathrm{~s}, 6 \mathrm{H}), 2.89$ (dd, $J=13.3,8.0 \mathrm{~Hz}, 1 \mathrm{H}), 2.71$ (dd, $J=$ 13.3, 8.0 Hz, 1H); $\left.{ }^{13} \mathbf{C ~ N M R ~ ( 1 0 1 ~} \mathrm{MHz}, \mathrm{CDCl}_{3}\right) \delta 174.9,161.0,146.7,143.6,138.4,137.5$, 134.1, 128.9, 128.7, 127.6, 127.5, 127.3, 126.8, 105.6, 98.7, 65.4, 55.4, 50.7, 45.6, 44.0. IR 3360 (w), 3331 (w), 3317 (w), 3306 (w), 3288 (w), 2997 (w), 2942 (w), 2903 (w), 2837 (w), 2361 (w), 2161 (s), 2051 (w), 1802 (w), 1650 (m), 1598 (s), 1525 (m), 1457 (m), 1429 (m), 1355 (w), 1324 (w), 1296 (w), 1274 (w), 1255 (w), 1205 (m), 1155 (s), 1082 (w), 1066 (w), $839(\mathrm{w}), 773(\mathrm{w}), 771(\mathrm{w}), 740(\mathrm{w}), 725(\mathrm{~m}), 698(\mathrm{~m}), 661(\mathrm{w})$.

HRMS (ESI/QTOF): Calcd for $\mathrm{C}_{27} \mathrm{H}_{28} \mathrm{NO}_{3}{ }^{+}[\mathrm{M}+\mathrm{H}]^{+}$414.2064; Found 414.2058. 
<smiles>COc1cc(F)cc(C2C=CC(C(=O)NCc3ccccc3)(c3ccccc3)C2)c1</smiles>

3ah

According to the general procedure, 3ah was obtained in $78 \%$ yield (31.3 $\mathrm{mg})$ as colorless oil with >19:1 dr and 95\% ee which was measured by SFC on Chiralcel IA (10\% $\mathrm{MeOH}$ in supercritical $\mathrm{CO}_{2}$ as eluent; flow rate $4.0 \mathrm{~mL} / \mathrm{min}$; $\mathrm{tR}$ (major) $=9.6 \mathrm{~min} ., \mathrm{tR}$ (minor) $=8.9 \mathrm{~min}$.). $[\alpha]_{D}^{25}+24.6\left(c 0.89, \mathrm{CHCl}_{3}\right)$.

${ }^{1} \mathbf{H}$ NMR (400 MHz, $\left.\mathrm{CDCl}_{3}\right) \delta$ 7.31-7.24 (m, 4H), 7.21-7.13 (m, 4H), $7.05(\mathrm{~d}, J=7.2 \mathrm{~Hz}, 2 \mathrm{H})$, $6.51(\mathrm{~s}, 1 \mathrm{H}), 6.48(\mathrm{~d}, J=9.5 \mathrm{~Hz}, 1 \mathrm{H}), 6.39(\mathrm{~d}, J=10.6 \mathrm{~Hz}, 1 \mathrm{H}), 6.12(\mathrm{dd}, J=5.7,2.5 \mathrm{~Hz}, 1 \mathrm{H})$, $5.94(\mathrm{~d}, J=5.3 \mathrm{~Hz}, 1 \mathrm{H}), 5.58$ (brs, $1 \mathrm{H}), 4.35$ (dd, $J=14.9,5.8 \mathrm{~Hz}, 1 \mathrm{H}), 4.30$ (dd, $J=14.9,5.8$ $\mathrm{Hz}, 1 \mathrm{H}), 3.80(\mathrm{t}, J=8.0 \mathrm{~Hz}, 1 \mathrm{H}), 3.68(\mathrm{~s}, 3 \mathrm{H}), 2.82(\mathrm{dd}, J=13.2,8.0 \mathrm{~Hz}, 1 \mathrm{H}), 2.60(\mathrm{dd}, J=$ 13.2, 8.0 Hz, 1H); ${ }^{13} \mathbf{C ~ N M R ~}\left(100 \mathrm{MHz}, \mathrm{CDCl}_{3}\right) \delta 174.7,163.8(\mathrm{~d}, J=244.0 \mathrm{~Hz}), 161.1(\mathrm{~d}, J=$ $11.0 \mathrm{~Hz}), 147.5$ (d, $J=8.0 \mathrm{~Hz}), 143.5,138.4,137.0,134.5,129.0,128.7,127.6,127.5,127.4$, 126.8, $109.1(\mathrm{~d}, J=2.6 \mathrm{~Hz}), 106.8(\mathrm{~d}, J=22.0 \mathrm{~Hz}), 99.8(\mathrm{~d}, J=25.0 \mathrm{~Hz}), 85.4,55.6,50.4$ (d, $J=2.0 \mathrm{~Hz}$ ), 45.5, 44.0. IR 3379 (w), 3360 (w), 3323 (w), 3059 (w), 3034 (w), 3032 (w), 2367 (w), 2161 (w), 2024 (w), 1995 (w), 1953 (w), 1916 (w), 1652 (s), 1617 (s), 1594 (s), 1529 (s), 1511 (m), 1496 (m), 1456 (m), 1454 (m), 1434 (m), 1336 (m), 1299 (m), 1193 (m), 1136 (s), $1057(\mathrm{w}), 1020(\mathrm{w}), 958$ (m), 846 (m), 829 (m), 806 (m), 779 (m), 760 (s), 744 (m), 733 (m), $696(\mathrm{~s}), 673(\mathrm{~m})$.

HRMS (ESI/QTOF): Calcd for $\mathrm{C}_{26} \mathrm{H}_{24} \mathrm{FNNaO}_{2}{ }^{+}[\mathrm{M}+\mathrm{Na}]^{+} 424.1683$; Found 424.1689.

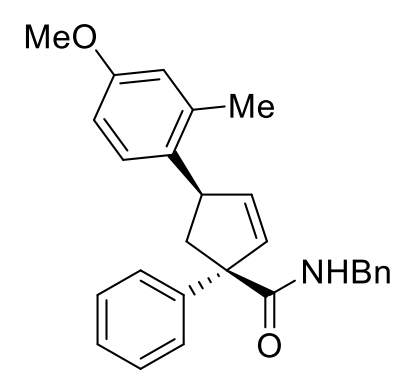

3ai

According to the general procedure, 3ai was obtained in $92 \%$ yield (36.5 mg) as colorless oil with >19:1 dr and $89 \%$ ee which was measured by SFC on Chiralcel IA $(10 \% \mathrm{MeOH}$ in supercritical $\mathrm{CO}_{2}$ as eluent; flow rate $4.0 \mathrm{~mL} / \mathrm{min}$; $\mathrm{tR}$ (major) $=13.2 \mathrm{~min} ., \mathrm{tR}$ (minor) $=12.1$ 
min.). $[\alpha]_{D}^{25}+37.3\left(c 1.75, \mathrm{CHCl}_{3}\right)$.

${ }^{1} \mathbf{H}$ NMR (400 MHz, $\left.\mathrm{CDCl}_{3}\right) \delta$ 7.33-7.26 (m, 4H), 7.23-7.17 (m, 4H), 7.07-7.05 (m, 3H), 6.65$6.62(\mathrm{~m}, 2 \mathrm{H}), 6.14(\mathrm{dd}, J=5.6,2.5 \mathrm{~Hz}, 1 \mathrm{H}), 5.98(\mathrm{dd}, J=5.6,2.0 \mathrm{~Hz}, 1 \mathrm{H}), 5.56(\mathrm{t}, J=5.7 \mathrm{~Hz}$, $1 \mathrm{H}), 4.37(\mathrm{dd}, J=14.9,5.7 \mathrm{~Hz}, 1 \mathrm{H}), 4.31(\mathrm{dd}, J=14.9,5.7 \mathrm{~Hz}, 1 \mathrm{H}), 4.03$ (tt, $J=7.9,2.3 \mathrm{~Hz}$, $1 \mathrm{H}), 3.71(\mathrm{~s}, 3 \mathrm{H}), 2.68(\mathrm{dd}, J=13.3,8.0 \mathrm{~Hz}, 1 \mathrm{H}), 2.64(\mathrm{dd}, J=13.2,7.8 \mathrm{~Hz}, 1 \mathrm{H}), 2.22(\mathrm{~s}, 3 \mathrm{H})$; ${ }^{13} \mathbf{C}$ NMR $\left(101 \mathrm{MHz}, \mathrm{CDCl}_{3}\right) \delta 175.0,158.0,143.9,138.5,137.8,136.9,134.8,133.7,128.9$, $128.7,128.0,127.6,127.5,127.2,126.7,116.0,111.5,65.4,55.3,46.0,44.9,43.9,20.0$. IR 3356 (w), 3333 (w), 3319 (w), 3317 (w), 3055 (w), 3053 (w), 3030 (w), 2945 (w), 2943 (w), 2926 (w), 2361 (w), 2161 (w), 2051 (w), 2014 (w), 1945 (w), 1801 (w), 1650 (s), 1610 (m), 1527 (s), 1525 (s), 1502 (s), 1452 (m), 1290 (m), 1255 (m), 1205 (w), 1163 (w), 1082 (w), 1049 (w), $816(\mathrm{w}), 800(\mathrm{w}), 798(\mathrm{w}), 758(\mathrm{w}), 740(\mathrm{w}), 723(\mathrm{~m}), 698(\mathrm{~m}), 660(\mathrm{w})$.

HRMS (nanochip-ESI/LTQ-Orbitrap): Calcd for $\mathrm{C}_{27} \mathrm{H}_{28} \mathrm{NO}_{2}{ }^{+}[\mathrm{M}+\mathrm{H}]^{+}$398.2115; Found 398.2127 .

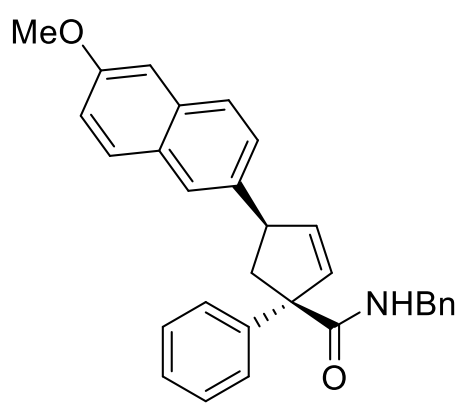

3aj

According to the general procedure, 3aj was obtained in $86 \%$ yield $(37.3 \mathrm{mg}$ ) as colorless oil with $>19: 1 \mathrm{dr}$ and $88 \%$ ee which was measured by SFC on Chiralcel IA $(20 \% \mathrm{MeOH}$ in supercritical $\mathrm{CO}_{2}$ as eluent; flow rate $4.0 \mathrm{~mL} / \mathrm{min}$; $\mathrm{tR}$ (major) $=13.1 \mathrm{~min}$., $\mathrm{tR}$ (minor) $=10.5$ $\min$.). $[\alpha]_{D}^{25}+107.4\left(c 0.94, \mathrm{CHCl}_{3}\right)$.

${ }^{1} \mathbf{H}$ NMR $\left(400 \mathrm{MHz}, \mathrm{CDCl}_{3}\right) \delta$ 7.61-7.53 (m, 2H), $7.50(\mathrm{~d}, J=2.0 \mathrm{~Hz}, 1 \mathrm{H}), 7.33-7.25(\mathrm{~m}, 5 \mathrm{H})$, 7.22-7.12 (m, 4H), 7.08-7.02 (m, 4H), $6.15(\mathrm{dd}, J=5.6,2.8 \mathrm{~Hz}, 1 \mathrm{H}), 6.03(\mathrm{dd}, J=5.6,1.6 \mathrm{~Hz}$, $1 \mathrm{H}), 5.62(\mathrm{t}, J=5.7 \mathrm{~Hz}, 1 \mathrm{H}), 4.36(\mathrm{dd}, J=14.9,5.8 \mathrm{~Hz}, 1 \mathrm{H}), 4.31(\mathrm{dd}, J=14.9,5.8 \mathrm{~Hz}, 1 \mathrm{H})$, $3.98(\mathrm{tt}, J=8.0,2.3 \mathrm{~Hz}, 1 \mathrm{H}), 3.82(\mathrm{~s}, 3 \mathrm{H}), 2.89$ (dd, $J=13.2,8.0 \mathrm{~Hz}, 1 \mathrm{H}), 2.69$ (dd, $J=13.2$, $8.0 \mathrm{~Hz}, 1 \mathrm{H}) ;{ }^{13} \mathrm{C}$ NMR $\left(101 \mathrm{MHz}, \mathrm{CDCl}_{3}\right) \delta$ 175.0, 157.5, 143.7, 139.3, 138.4, 137.9, 134.1, 133.5, 129.2, 129.1, 128.9, 128.7, 127.6, 127.5, 127.33, 127.27, 126.9, 126.8, 125.7, 118.9, 105.8, 65.5, 55.4, 50.5, 45.9, 44.0. IR 3352 (w), 3323 (w), 3055 (w), 3024 (w), 3022 (w), 2947 (w), 2936 (w), 2159 (w), 1652 (s), 1606 (s), 1508 (s), 1454 (m), 1392 (w), 1269 (s), 1236 (m), $1215(\mathrm{~m}), 1163(\mathrm{~m}), 1078(\mathrm{w}), 1033(\mathrm{~m}), 962$ (w), $962(\mathrm{w}), 899(\mathrm{w}), 854(\mathrm{~m}), 835(\mathrm{w}), 806(\mathrm{w})$, 
$785(\mathrm{w}), 758(\mathrm{~s}), 740(\mathrm{~m}), 712(\mathrm{~m}), 696(\mathrm{~s})$.

HRMS (ESI/QTOF): Calcd for $\mathrm{C}_{30} \mathrm{H}_{28} \mathrm{NO}_{2}{ }^{+}[\mathrm{M}+\mathrm{H}]^{+} 434.2115$; Found 434.2109.

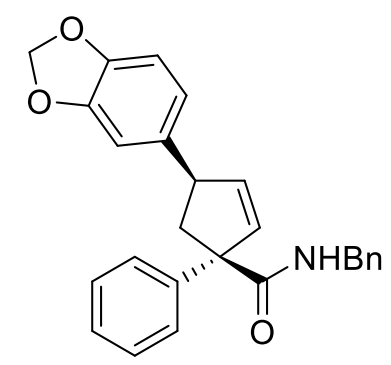

3ak

According to the general procedure, 3ak was obtained in $75 \%$ yield $(29.8 \mathrm{mg})$ as colorless oil with >19:1 dr and $88 \%$ ee which was measured by SFC on Chiralcel $\mathrm{OJ}-\mathrm{H}(10 \% \mathrm{MeOH}$ in supercritical $\mathrm{CO}_{2}$ as eluent; flow rate $4.0 \mathrm{~mL} / \mathrm{min}$; $\mathrm{tR}$ (major) $=10.8 \mathrm{~min}$., $\mathrm{tR}$ (minor) $=12.8$ min.). $[\alpha]_{D}^{25}+49.8\left(c 0.92, \mathrm{CHCl}_{3}\right)$.

${ }^{1} \mathbf{H}$ NMR (400 MHz, $\left.\mathrm{CDCl}_{3}\right) \delta$ 7.30-7.24 (m, 4H), 7.22-7.13 (m, 4H), 7.07-7.05 (m, 2H), 6.67 $(\mathrm{d}, J=1.7 \mathrm{~Hz}, 1 \mathrm{H}), 6.64(\mathrm{~d}, J=7.9 \mathrm{~Hz}, 1 \mathrm{H}), 6.60(\mathrm{dd}, J=8.0,1.7 \mathrm{~Hz}, 1 \mathrm{H}), 6.09(\mathrm{dd}, J=5.6$, $2.6 \mathrm{~Hz}, 1 \mathrm{H}), 5.92(\mathrm{dd}, J=5.5,2.0 \mathrm{~Hz}, 1 \mathrm{H}), 5.83(\mathrm{~s}, 2 \mathrm{H}), 5.58(\mathrm{t}, J=5.7 \mathrm{~Hz}, 1 \mathrm{H}), 4.36$ (dd, $J=$ 14.9, $5.8 \mathrm{~Hz}, 1 \mathrm{H}), 4.30$ (dd, $J=14.9,5.8 \mathrm{H} \mathrm{z}, 1 \mathrm{H}), 3.78$ (tt, $J=8.0,2.3 \mathrm{~Hz}, 1 \mathrm{H}), 2.77$ (dd, $J=$ $13.2,7.9 \mathrm{~Hz}, 1 \mathrm{H}), 2.60(\mathrm{dd}, J=13.2,8.0 \mathrm{~Hz}, 1 \mathrm{H}) ;{ }^{13} \mathbf{C ~ N M R}\left(101 \mathrm{MHz}, \mathrm{CDCl}_{3}\right) \delta 174.9,147.9$, $146.2,143.7,138.4,138.3,137.9,133.9,128.9,128.7,127.6,127.5,127.3,126.8,120.5,108.3$, 108.2, 101.0, 65.4, 50.2, 46.1, 44.0. IR 3356 (w), 3354 (w), 3325 (w), 3323 (w), 3063 (w), 3022 (w), $2886(\mathrm{w}), 2155(\mathrm{w}), 1953$ (w), 1951 (w), 1652 (s), 1504 (s), 1490 (s), 1446 (m), 1247 (s), 1041 (m), $935(\mathrm{w}), 798(\mathrm{~m}), 769(\mathrm{w}), 750(\mathrm{w}), 733(\mathrm{~m}), 698(\mathrm{~s})$.

HRMS (ESI/QTOF): Calcd for $\mathrm{C}_{26} \mathrm{H}_{24} \mathrm{NO}_{3}{ }^{+}[\mathrm{M}+\mathrm{H}]^{+}$398.1751; Found 398.1752.

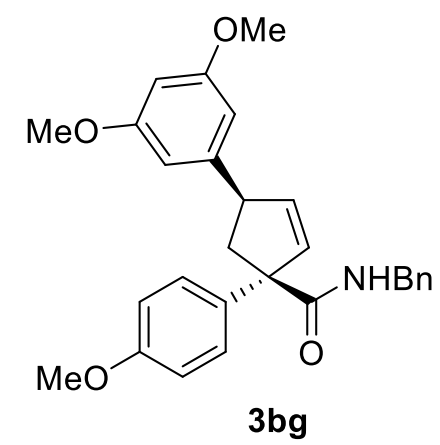

According to the general procedure, $\mathbf{3 b g}$ was obtained in 66\% yield (29.2 $\mathrm{mg})$ as colorless oil with >19:1 dr and 97\% ee which was measured by SFC on Chiralcel IA (10\% $\mathrm{MeOH}$ in supercritical $\mathrm{CO}_{2}$ as eluent; flow rate $4.0 \mathrm{~mL} / \mathrm{min}$; $\mathrm{tR}$ (major) $=17.1 \mathrm{~min}$., $\mathrm{tR}$ (minor) $=16.0$ 
min.). $[\alpha]_{D}^{25}+94.0\left(c 1.37, \mathrm{CHCl}_{3}\right)$.

${ }^{1} \mathbf{H}$ NMR $\left(400 \mathrm{MHz}, \mathrm{CDCl}_{3}\right) \delta$ 7.29-7.23 (m, 5H), $7.13(\mathrm{~d}, J=6.8 \mathrm{~Hz}, 2 \mathrm{H}), 6.89-6.84(\mathrm{~m}, 2 \mathrm{H})$, $6.42(\mathrm{~d}, J=2.3 \mathrm{~Hz}, 2 \mathrm{H}), 6.32(\mathrm{t}, J=2.3 \mathrm{~Hz}, 1 \mathrm{H}), 6.18(\mathrm{dd}, J=5.8,2.5 \mathrm{~Hz}, 1 \mathrm{H}), 6.03(\mathrm{dd}, J=$ $5.5,1.9 \mathrm{~Hz}, 1 \mathrm{H}), 5.70$ (brs, 1H), $4.43(\mathrm{dd}, J=15.0,5.7 \mathrm{~Hz}, 1 \mathrm{H}), 4.38(\mathrm{dd}, J=14.9,5.8 \mathrm{~Hz}, 1 \mathrm{H})$, 3.87-3.82 (m, 1H), $3.79(\mathrm{~s}, 3 \mathrm{H}), 3.76(\mathrm{~s}, 6 \mathrm{H}), 2.89(\mathrm{dd}, J=13.1,8.2 \mathrm{~Hz}, 1 \mathrm{H}), 2.66(\mathrm{dd}, J=13.1$, $7.9 \mathrm{~Hz}, 1 \mathrm{H}) ;{ }^{13} \mathbf{C}$ NMR $\left(101 \mathrm{MHz}, \mathrm{CDCl}_{3}\right) \delta 175.2,161.0,158.8,146.8,138.5,137.3,135.5$, 134.5, 128.8, 128.0, 127.6, 127.5, 114.3, 105.6, 98.7, 64.7, 55.4, 50.7, 45.8, 44.0. IR 3364 (w), 3344 (w), 3325 (w), 2990 (w), 2988 (w), 2957 (m), 2905 (w), 2837 (w), 2363 (w), 2040 (w), 1928 (w), 1652 (m), 1602 (s), 1600 (s), 1511 (s), 1459 (m), 1430 (m), 1355 (w), 1330 (w), 1296 (m), 1251 (s), 1205 (m), 1184 (m), 1155 (s), 1064 (m), 931 (w), 833 (m), 804 (w), 791 (w), 769 (w), $756(\mathrm{w}), 733(\mathrm{w}), 694(\mathrm{~m}), 692(\mathrm{~m}), 673(\mathrm{w})$.

HRMS (nanochip-ESI/LTQ-Orbitrap): Calcd for $\mathrm{C}_{28} \mathrm{H}_{30} \mathrm{NO}_{4}{ }^{+}[\mathrm{M}+\mathrm{H}]^{+}$444.2169; Found 444.2179.

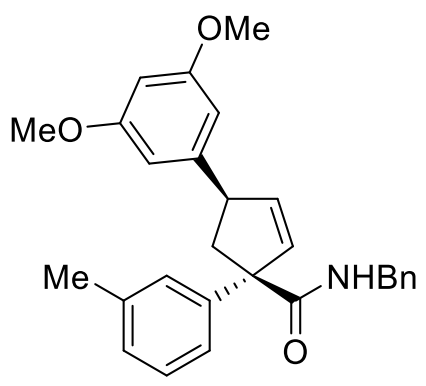

$3 \mathrm{cg}$

According to the general procedure, 3cg was obtained in $63 \%$ yield $(27.0 \mathrm{mg})$ as colorless oil with $10: 1 \mathrm{dr}$ and $89 \%$ ee which was measured by SFC on Chiralcel IA (10\% $\mathrm{MeOH}$ in supercritical $\mathrm{CO}_{2}$ as eluent; flow rate $4.0 \mathrm{~mL} / \mathrm{min}$; $\mathrm{tR}$ (major) $=10.1 \mathrm{~min}$., $\mathrm{tR}$ (minor) $=9.2$ min.). $[\alpha]_{D}^{25}+76.8\left(c 1.28, \mathrm{CHCl}_{3}\right)$.

${ }^{1} \mathbf{H}$ NMR (400 MHz, $\left.\mathrm{CDCl}_{3}\right) \delta$ 7.29-7.21 (m, 4H), 7.17-7.12 (m, 4H), $7.08(\mathrm{~d}, J=7.4 \mathrm{~Hz}, 1 \mathrm{H})$, $6.43(\mathrm{~d}, J=2.2 \mathrm{~Hz}, 2 \mathrm{H}), 6.33(\mathrm{t}, J=2.2 \mathrm{~Hz}, 1 \mathrm{H}), 6.18(\mathrm{dd}, J=5.5,2.5 \mathrm{~Hz}, 1 \mathrm{H}), 6.04(\mathrm{dd}, J=$ $5.5,1.7 \mathrm{~Hz}, 1 \mathrm{H}), 5.63$ (brs, $1 \mathrm{H}), 4.42(\mathrm{~d}, J=5.7 \mathrm{~Hz}, 2 \mathrm{H}), 3.87(\mathrm{tt}, J=8.0,2.1 \mathrm{~Hz}, 1 \mathrm{H}), 3.77$ (s, $6 \mathrm{H}), 2.89(\mathrm{dd}, J=13.2,8.0 \mathrm{~Hz}, 1 \mathrm{H}), 2.68(\mathrm{dd}, J=13.2,8.0 \mathrm{~Hz}, 1 \mathrm{H}), 2.34(\mathrm{~s}, 3 \mathrm{H}) ;{ }^{13} \mathbf{C ~ N M R}$ $\left(101 \mathrm{MHz}, \mathrm{CDCl}_{3}\right) \delta 174.9,161.1,146.9,143.6,138.6,138.5,137.4,134.3,128.8,128.75$, 128.1, 127.6, 127.5, 123.7, 105.6, 98.8, 65.4, 55.5, 50.7, 45.5, 44.0, 21.7. IR 3358 (m), 3333 (m), 3034 (m), 2947 (m), 2945 (m), 2361 (m), 2340 (m), 2022 (w), 1772 (w), 1652 (s), 1600 (s), 1598 (s), 1519 (s), 1459 (m), 1429 (m), 1353 (w), 1351 (w), 1328 (w), 1326 (w), 1294 (w), 1292 (w), 1265 (w), 1205 (m), 1155 (s), 1066 (w), 1066 (w), 1028 (w), $1012(\mathrm{w}), 839$ (w), 839 
(w), $793(\mathrm{w}), 791(\mathrm{w}), 775(\mathrm{w}), 760(\mathrm{w}), 740(\mathrm{w}), 723(\mathrm{w}), 700(\mathrm{w})$.

HRMS (ESI/QTOF): Calcd for $\mathrm{C}_{28} \mathrm{H}_{29} \mathrm{NNaO}_{3}{ }^{+}[\mathrm{M}+\mathrm{Na}]^{+} 450.2040$; Found 450.2041.

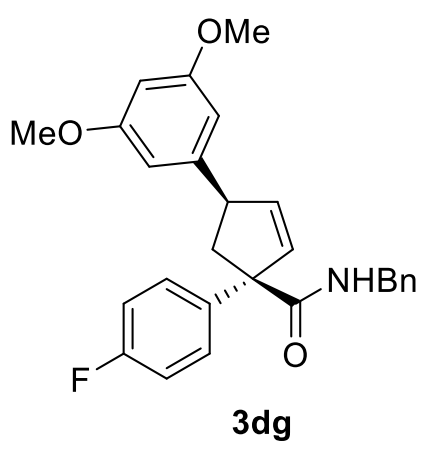

According to the general procedure, $\mathbf{3 d g}$ was obtained in $67 \%$ yield $(29.1 \mathrm{mg})$ as colorless oil with 17:1 dr and 91\% ee which was measured by SFC on Chiralcel IC (10\% $\mathrm{MeOH}$ in supercritical $\mathrm{CO}_{2}$ as eluent; flow rate $4.0 \mathrm{~mL} / \mathrm{min}$; $\mathrm{tR}$ (major) $=10.8 \mathrm{~min}$., $\mathrm{tR}$ (minor) $=14.1$ $\min$.). $[\alpha]_{D}^{25}+75.9\left(c 1.33, \mathrm{CHCl}_{3}\right)$.

${ }^{1} \mathbf{H}$ NMR $\left(400 \mathrm{MHz}, \mathrm{CDCl}_{3}\right) \delta$ 7.35-7.22 (m, 5H), $7.13(\mathrm{~d}, J=6.7 \mathrm{~Hz}, 2 \mathrm{H}), 7.02(\mathrm{t}, J=8.6 \mathrm{~Hz}$, 2H), $6.40(\mathrm{~d}, J=2.0 \mathrm{~Hz}, 2 \mathrm{H}), 6.33(\mathrm{t}, J=1.9 \mathrm{~Hz}, 1 \mathrm{H}), 6.17(\mathrm{dd}, J=5.5,2.4 \mathrm{~Hz}, 1 \mathrm{H}), 6.07$ (dd, $J=5.5,1.9 \mathrm{~Hz}, 1 \mathrm{H}), 5.70(\mathrm{t}, J=5.7 \mathrm{~Hz}, 1 \mathrm{H}), 4.42(\mathrm{dd}, J=14.9,5.7 \mathrm{~Hz}, 1 \mathrm{H}), 4.39(\mathrm{dd}, J=14.9$, $5.7 \mathrm{~Hz}, 1 \mathrm{H}), 3.86-3.84(\mathrm{~m}, 1 \mathrm{H}), 3.76$ (s, 6H), 2.85 (dd, $J=13.3,7.9 \mathrm{~Hz}, 1 \mathrm{H}), 2.66$ (dd, $J=13.3$, $8.0 \mathrm{~Hz}, 1 \mathrm{H}), 2.34(\mathrm{~s}, 3 \mathrm{H}) ;{ }^{13} \mathrm{C}$ NMR $\left(101 \mathrm{MHz}, \mathrm{CDCl}_{3}\right) \delta 174.6,162.0$ (d, $\left.J=246.2 \mathrm{~Hz}\right), 161.0$, $146.5,139.3(\mathrm{~d}, J=3.2 \mathrm{~Hz}), 138.28,137.9,133.9,128.8,128.4$ (d, $J=8.0 \mathrm{~Hz}), 127.61,127.57$, $115.6(\mathrm{~d}, J=21.3 \mathrm{~Hz}), 105.5,98.7,64.7,55.4,50.6,45.9,44.0$. IR 3354 (w), $3352(\mathrm{w}), 3327$ (w), 3308 (w), 3306 (w), 2940 (w), 2938 (w), 2837 (w), 2022 (w), 1650 (m), 1598 (s), 1508 (s), 1459 (m), 1430 (m), 1353 (w), 1328 (w), 1326 (w), 1294 (w), 1269 (w), 1230 (m), 1205 (m), 1155 (s), 1066 (m), 1001 (w), 929 (w), 887 (w), 837 (m), 791 (w), 779 (w), 760 (m), 733 (w), $702(\mathrm{~m}), 690(\mathrm{~m}), 673(\mathrm{w}), 660(\mathrm{w})$.

HRMS (ESI/QTOF): Calcd for $\mathrm{C}_{27} \mathrm{H}_{26} \mathrm{FNNaO}_{3}{ }^{+}[\mathrm{M}+\mathrm{Na}]^{+} 454.1789$; Found 454.1789.

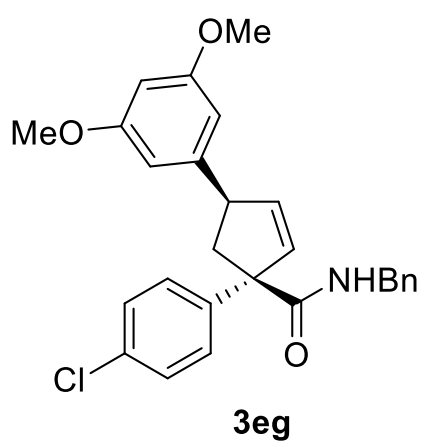

According to the general procedure, $3 \mathbf{e g}$ was obtained in $63 \%$ yield $(28.4 \mathrm{mg})$ as colorless oil 
with >19:1 dr and $84 \%$ ee which was measured by SFC on Chiralcel IA (15\% $\mathrm{MeOH}$ in supercritical $\mathrm{CO}_{2}$ as eluent; flow rate $4.0 \mathrm{~mL} / \mathrm{min}$; $\mathrm{tR}$ (major) $=10.4 \mathrm{~min} ., \mathrm{tR}$ (minor) $=8.0$ $\min$.). $[\alpha]_{D}^{25}+103.1\left(\right.$ c $\left.1.30, \mathrm{CHCl}_{3}\right)$.

${ }^{1} \mathbf{H}$ NMR (400 MHz, $\left.\mathrm{CDCl}_{3}\right) \delta$ 7.31-7.25 (m, 7H), 7.16-7.14 (m, 2H), 6.39 (d, J=2.2 Hz, 2H), $6.33(\mathrm{t}, J=2.2 \mathrm{~Hz}, 1 \mathrm{H}), 6.15(\mathrm{dd}, J=5.6,2.5 \mathrm{~Hz}, 1 \mathrm{H}), 6.08(\mathrm{dd}, J=5.5,1.8 \mathrm{~Hz}, 1 \mathrm{H}), 5.65(\mathrm{t}, J$ $=5.7 \mathrm{~Hz}, 1 \mathrm{H}), 4.44(\mathrm{dd}, J=14.8,5.7 \mathrm{~Hz}, 1 \mathrm{H}), 4.39(\mathrm{dd}, J=14.8,5.7 \mathrm{~Hz}, 1 \mathrm{H}), 3.86(\mathrm{tt}, J=8.0$, $2.2 \mathrm{~Hz}, 1 \mathrm{H}), 3.76(\mathrm{~s}, 6 \mathrm{H}), 2.85(\mathrm{dd}, J=13.3,7.9 \mathrm{~Hz}, 1 \mathrm{H}), 2.66(\mathrm{dd}, J=13.3,8.0 \mathrm{~Hz}, 1 \mathrm{H}) ;{ }^{13} \mathrm{C}$ NMR $\left(101 \mathrm{MHz}, \mathrm{CDCl}_{3}\right) \delta 174.3,161.1,146.4,142.1,138.3,138.2,133.6,133.2,129.0,128.8$, 128.2, 127.68, 127.65, 105.6, 98.7, 64.9, 55.5, 50.7, 45.9, 44.1. IR 3350 (w), 3337 (w), 2955 (w), 2940 (w), 2837 (w), 2161 (w), 1650 (m), 1596 (s), 1523 (m), 1492 (m), 1459 (m), 1429 (m), 1353 (w), $1328(\mathrm{w}), 1294(\mathrm{w}), 1205$ (m), 1155 (s), 1095 (w), $1066(\mathrm{~m}), 1014(\mathrm{w}), 929(\mathrm{w})$, $831(\mathrm{~m}), 783(\mathrm{w}), 760(\mathrm{~m}), 733(\mathrm{w}), 700(\mathrm{~m}), 669(\mathrm{w})$.

HRMS (ESI/QTOF): Calcd for $\mathrm{C}_{27} \mathrm{H}_{26} \mathrm{ClNNaO}_{3}{ }^{+}[\mathrm{M}+\mathrm{Na}]^{+}$470.1493; Found 470.1492.

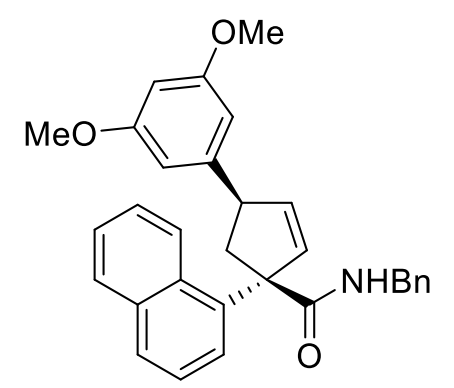

$3 f g$

According to the general procedure, $\mathbf{3 f g}$ was obtained in $65 \%$ yield (30.3 $\mathrm{mg}$ ) as colorless oil with $>19: 1 \mathrm{dr}$ and $92 \%$ ee which was measured by SFC on Chiralcel AS-H $(10 \% \mathrm{MeOH}$ in supercritical $\mathrm{CO}_{2}$ as eluent; flow rate $4.0 \mathrm{~mL} / \mathrm{min}$; $\mathrm{tR}$ (major) $=7.5 \mathrm{~min}$., $\mathrm{tR}$ (minor) $=5.5 \mathrm{~min}$.). $[\alpha]_{D}^{25}-72.6\left(c 1.40, \mathrm{CHCl}_{3}\right)$.

${ }^{1} \mathbf{H}$ NMR $\left(400 \mathrm{MHz}, \mathrm{CDCl}_{3}\right) \delta$ 7.89-7.86 (m, 2H), $7.80(\mathrm{~d}, J=8.2 \mathrm{~Hz}, 1 \mathrm{H}), 7.55-7.38(\mathrm{~m}, 4 \mathrm{H})$, 7.13-7.09 (m, 3H), 6.87-6.84 (m, 2H), $6.53(\mathrm{~d}, J=2.3 \mathrm{~Hz}, 2 \mathrm{H}), 6.33(\mathrm{t}, J=2.2 \mathrm{~Hz}, 1 \mathrm{H}), 6.18$ $(\mathrm{dd}, J=5.5,2.6 \mathrm{~Hz}, 1 \mathrm{H}), 6.14(\mathrm{dd}, J=5.5,1.6 \mathrm{~Hz}, 1 \mathrm{H}), 5.51(\mathrm{t}, J=5.9 \mathrm{~Hz}, 1 \mathrm{H}), 4.36(\mathrm{dd}, J=$ 15.1, 5.7 Hz, 1H), $4.30(\mathrm{dd}, J=15.1,6.0 \mathrm{~Hz}, 1 \mathrm{H}), 3.86-3.82(\mathrm{~m}, 1 \mathrm{H}), 3.78(\mathrm{~s}, 6 \mathrm{H}), 3.55(\mathrm{dd}, J$ $=13.2,8.0 \mathrm{~Hz}, 1 \mathrm{H}), 2.68(\mathrm{dd}, J=13.2,8.4 \mathrm{~Hz}, 1 \mathrm{H}) ;{ }^{13} \mathbf{C ~ N M R}\left(101 \mathrm{MHz}, \mathrm{CDCl}_{3}\right) \delta 175.0$, 161.0, 147.1, 140.0, 138.6, 138.3, 134.7, 134.3, 131.2, 129.2, 128.9, 128.5, 127.4, 127.3, 126.7, 126.1, 125.4, 125.2, 124.9, 105.8, 98.9, 65.7, 55.5, 51.3, 44.0, 43.8. IR 2947 (m), 2361 (m), 1669 (m), 1654 (m), 1598 (s), 1509 (s), 1459 (m), 1430 (m), 1351 (w), 1205 (m), 1153 (s), 1064 $(\mathrm{w}), 827(\mathrm{w}), 810(\mathrm{w}), 783(\mathrm{w}), 756(\mathrm{w}), 700(\mathrm{w}), 658(\mathrm{w})$. 
HRMS (ESI/QTOF): Calcd for $\mathrm{C}_{31} \mathrm{H}_{29} \mathrm{NNaO}_{3}{ }^{+}[\mathrm{M}+\mathrm{Na}]^{+}$486.2040; Found 486.2048.

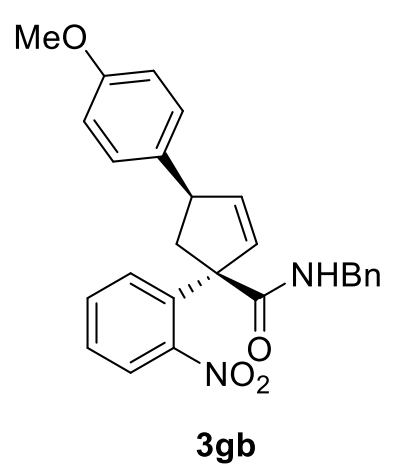

According to the general procedure, 3gb was obtained in $55 \%$ yield $(23.5 \mathrm{mg})$ as pale yellow oil with $10: 1 \mathrm{dr}$ and $68 \%$ ee which was measured by SFC on Chiralcel AS-H $(10 \% \mathrm{MeOH}$ in supercritical $\mathrm{CO}_{2}$ as eluent; flow rate $4.0 \mathrm{~mL} / \mathrm{min}$; $\mathrm{tR}$ (major) $=5.8 \mathrm{~min}$., $\mathrm{tR}$ (minor) $=4.8 \mathrm{~min}$.). $[\alpha]_{D}^{25}+29.3\left(c 1.15, \mathrm{CHCl}_{3}\right)$.

${ }^{1} \mathbf{H}$ NMR $\left(400 \mathrm{MHz}, \mathrm{CDCl}_{3}\right) \delta 7.97(\mathrm{~d}, J=7.8 \mathrm{~Hz}, 1 \mathrm{H}), 7.62-7.59(\mathrm{~m}, 2 \mathrm{H}), 7.46-7.42(\mathrm{~m}, 1 \mathrm{H})$, 7.33-7.21 (m, 5H), $7.12(\mathrm{~d}, J=8.6 \mathrm{~Hz}, 2 \mathrm{H}), 6.79(\mathrm{~d}, J=8.6 \mathrm{~Hz}, 2 \mathrm{H}), 6.17-6.13(\mathrm{~m}, 2 \mathrm{H}), 5.69$ (t, $J=5.6 \mathrm{~Hz}, 1 \mathrm{H}), 4.41(\mathrm{dd}, J=14.7,5.6 \mathrm{~Hz}, 1 \mathrm{H}), 4.36(\mathrm{dd}, J=14.7,5.4 \mathrm{~Hz}, 1 \mathrm{H}), 4.09-4.05$ $(\mathrm{m}, 1 \mathrm{H}), 3.78(\mathrm{~s}, 3 \mathrm{H}), 2.86(\mathrm{~d}, J=7.9 \mathrm{~Hz}, 2 \mathrm{H}) ;{ }^{13} \mathbf{C ~ N M R}\left(101 \mathrm{MHz}, \mathrm{CDCl}_{3}\right) \delta 172.8,158.6$, 148.8, 139.6, 138.6, 138.3, 136.0, 134.1, 133.3, 129.3, 128.8, 128.4, 128.3, 128.1, 127.6, 125.8, 114.3, 65.5, 55.4, 49.8, 46.6, 44.2. IR 1668 (m), 1610 (w), 1527 (s), 1513 (s), 1456 (w), 1357 (w), $1355(\mathrm{w}), 1303(\mathrm{w}), 1247(\mathrm{~m}), 1180(\mathrm{w}), 1037(\mathrm{w}), 1037(\mathrm{w}), 1012(\mathrm{w}), 1012(\mathrm{w}), 835(\mathrm{w})$, $816(\mathrm{w}), 814(\mathrm{w}), 793(\mathrm{w}), 754(\mathrm{w}), 737(\mathrm{w}), 710(\mathrm{w}), 700(\mathrm{w}), 658(\mathrm{w})$.

HRMS (ESI/QTOF): Calcd for $\mathrm{C}_{26} \mathrm{H}_{25} \mathrm{~N}_{2} \mathrm{O}_{4}{ }^{+}[\mathrm{M}+\mathrm{H}]^{+} 429.1809$; Found 429.1801.<smiles>COc1cc(OC)cc(C2C=CC(Cc3ccccc3)(C(=O)Nc3ccccc3)C2)c1</smiles>

3hg

According to the general procedure, $3 \mathbf{h g}$ was obtained in $50 \%$ yield $(21.5 \mathrm{mg})$ as colorless oil with >19:1 dr and 95\% ee which was measured by SFC on Chiralcel IC $(10 \% \mathrm{MeOH}$ in supercritical $\mathrm{CO}_{2}$ as eluent; flow rate $4.0 \mathrm{~mL} / \mathrm{min}$; $\mathrm{tR}$ (major) $=15.2 \mathrm{~min}$., $\mathrm{tR}$ (minor) $=13.6$ min.). $[\alpha]_{D}^{25}+68.3\left(c 1.05, \mathrm{CHCl}_{3}\right)$. 
${ }^{1} \mathbf{H}$ NMR $\left(400 \mathrm{MHz}, \mathrm{CDCl}_{3}\right) \delta$ 7.26-7.19 (m, 8H), 7.09-7.07 (m, 2H), $6.30(\mathrm{t}, J=2.0 \mathrm{~Hz}, 1 \mathrm{H})$, $6.25(\mathrm{~d}, J=2.1 \mathrm{~Hz}, 2 \mathrm{H}), 5.97(\mathrm{dd}, J=5.6,1.8 \mathrm{~Hz}, 1 \mathrm{H}), 5.79(\mathrm{dd}, J=5.6,2.1 \mathrm{~Hz}, 1 \mathrm{H}), 5.70$ (t, $J=5.6 \mathrm{~Hz}, 1 \mathrm{H}), 4.41(\mathrm{dd}, J=14.8,5.7 \mathrm{~Hz}, 1 \mathrm{H}), 4.33(\mathrm{dd}, J=14.8,5.5 \mathrm{~Hz}, 1 \mathrm{H}), 3.77-3.74(\mathrm{~m}$, 1H), $3.73(\mathrm{~s}, 6 \mathrm{H}), 3.40(\mathrm{~d}, J=12.9 \mathrm{~Hz}, 1 \mathrm{H}), 2.86(\mathrm{~d}, J=12.9 \mathrm{~Hz}, 1 \mathrm{H}), 2.62(\mathrm{dd}, J=13.5,8.6$ $\mathrm{Hz}, 1 \mathrm{H}), 2.08(\mathrm{dd}, J=13.6,7.4 \mathrm{~Hz}, 1 \mathrm{H}) ;{ }^{13} \mathrm{C}$ NMR $\left(101 \mathrm{MHz}, \mathrm{CDCl}_{3}\right) \delta 175.7,161.1,146.8$, 138.6, 138.3, 138.2, 133.4, 130.4, 128.8, 128.2, 127.8, 127.5, 126.5, 105.4, 98.3, 61.8, 55.4, 50.4, 45.0, 43.8, 43.7. IR 3339 (w), 3325 (w), 3323 (w), 3030 (w), 2957 (w), 2938 (w), 2936 (w), 2837 (w), 1648 (m), 1596 (s), 1519 (m), 1498 (m), 1457 (m), 1429 (m), 1348 (w), 1330 (w), $1330(\mathrm{w}), 1294$ (w), 1205 (s), 1155 (s), 1066 (m), 837 (m), 775 (w), 760 (s), 746 (m), 702 (s), $677(\mathrm{w}), 661(\mathrm{w})$.

HRMS (ESI/QTOF): Calcd for $\mathrm{C}_{28} \mathrm{H}_{29} \mathrm{NNaO}_{3}{ }^{+}[\mathrm{M}+\mathrm{Na}]^{+} 450.2040$; Found 450.2036.

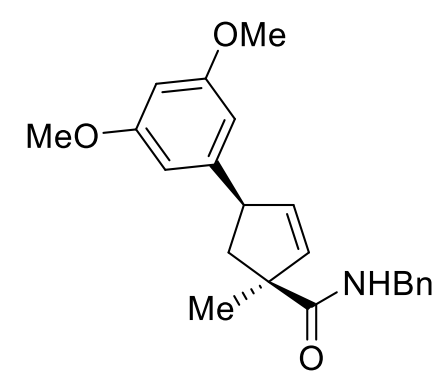

$3 i g$

According to the general procedure, 3ig was obtained in $61 \%$ yield $(21.5 \mathrm{mg})$ as colorless oil with >19:1 dr and 95\% ee which was measured by SFC on Chiralcel IA (5\% $\mathrm{MeOH}$ in supercritical $\mathrm{CO}_{2}$ as eluent; flow rate $4.0 \mathrm{~mL} / \mathrm{min}$; $\mathrm{tR}$ (major) $=11.5 \mathrm{~min}$., $\mathrm{tR}$ (minor) $=13.4$ $\min$.). $[\alpha]_{D}^{25}-5.2\left(c 1.10, \mathrm{CHCl}_{3}\right)$.

${ }^{1} \mathbf{H}$ NMR $\left(400 \mathrm{MHz}, \mathrm{CDCl}_{3}\right) \delta$ 7.32-7.25 (m, 3H), 7.20-7.19 (m, 2H), 6.30 (s, 3H), 5.93-5.88 $(\mathrm{m}, 3 \mathrm{H}), 4.42(\mathrm{dd}, J=14.8,5.7 \mathrm{~Hz}, 1 \mathrm{H}), 4.38(\mathrm{dd}, J=14.8,5.6 \mathrm{~Hz}, 1 \mathrm{H}), 4.03-3.98(\mathrm{~m}, 1 \mathrm{H})$, $3.72(\mathrm{~s}, 6 \mathrm{H}), 2.41(\mathrm{dd}, J=13.3,8.6 \mathrm{~Hz}, 1 \mathrm{H}), 2.13(\mathrm{dd}, J=13.3,7.3 \mathrm{~Hz}, 1 \mathrm{H}), 1.40(\mathrm{~s}, 3 \mathrm{H}) ;{ }^{13} \mathrm{C}$ NMR $\left(101 \mathrm{MHz}, \mathrm{CDCl}_{3}\right) \delta 176.8,161.1,147.1,138.5,136.5,136.3,128.8,127.7,127.5,105.3$, 98.4, 56.1, 55.4, 50.4, 46.5, 43.8, 24.0. IR 3354 (m), 3329 (m), 2363 (m), 2340 (m), 1683 (m), 1650 (s), 1596 (s), 1538 (s), 1523 (s), 1459 (s), 1457 (s), 1429 (m), 1353 (m), 1324 (m), 1205 (m), 1157 (s), $1010(\mathrm{w}), 991(\mathrm{w}), 976(\mathrm{w}), 939$ (w), 937 (w), $870(\mathrm{w}), 835(\mathrm{w}), 798(\mathrm{w}), 771$ (w), $756(\mathrm{w}), 723(\mathrm{~m}), 708(\mathrm{w}), 681(\mathrm{w}), 661(\mathrm{w})$.

HRMS (ESI/QTOF): Calcd for $\mathrm{C}_{22} \mathrm{H}_{26} \mathrm{NO}_{3}{ }^{+}[\mathrm{M}+\mathrm{H}]^{+} 352.1907$; Found 352.1907. 
<smiles>COc1cc(OC)cc(C2C=CC(CC(=O)OC(C)(C)C)(C(=O)NCc3ccccc3)C2)c1</smiles>

According to the general procedure, $\mathbf{3 j g}$ was obtained in $70 \%$ yield ( $94.3 \mathrm{mg}, 0.3 \mathrm{mmol} \mathrm{scale})$ as colorless oil with >19:1 dr and 97\% ee which was measured by SFC on Chiralcel AD-H (7\% $\mathrm{MeOH}$ in supercritical $\mathrm{CO}_{2}$ as eluent; flow rate $4.0 \mathrm{~mL} / \mathrm{min}$; $\mathrm{tR}$ (major) $=9.3 \mathrm{~min}$., $\mathrm{tR}$ (minor) $=14.7$ min. $)$. $[\alpha]_{D}^{25}+64.1\left(c 0.34, \mathrm{CHCl}_{3}\right)$.

${ }^{1} \mathbf{H}$ NMR $\left(400 \mathrm{MHz}, \mathrm{CDCl}_{3}\right) \delta$ 7.30-7.21 (m, 5H), 6.30-6.28 (m, 3H), $6.15(\mathrm{dd}, J=5.5,2.2 \mathrm{~Hz}$, $1 \mathrm{H}), 6.08(\mathrm{t}, J=5.5 \mathrm{~Hz}, 1 \mathrm{H}), 6.03(\mathrm{dd}, J=5.5,1.8 \mathrm{~Hz}, 1 \mathrm{H}), 4.46(\mathrm{dd}, J=14.9,6.0 \mathrm{~Hz}, 1 \mathrm{H})$, $4.38(\mathrm{dd}, J=14.9,5.4 \mathrm{~Hz}, 1 \mathrm{H}), 3.96(\mathrm{t}, J=7.8 \mathrm{~Hz}, 1 \mathrm{H}), 3.71(\mathrm{~s}, 6 \mathrm{H}), 3.11(\mathrm{~d}, J=16.2 \mathrm{~Hz}, 1 \mathrm{H})$, $2.54(\mathrm{dd}, J=13.3,8.7 \mathrm{~Hz}, 1 \mathrm{H}), 2.51(\mathrm{~d}, J=16.0 \mathrm{~Hz}, 1 \mathrm{H}), 2.08(\mathrm{dd}, J=13.8,7.3 \mathrm{~Hz}, 1 \mathrm{H}), 1.44$ (s, 9H); ${ }^{13}$ C NMR (101 MHz, $\left.\mathrm{CDCl}_{3}\right) \delta 175.4,171.1,161.1,146.7,138.8,138.5,134.1,128.7$, 127.7, 127.4, 105.3, 98.4, 80.8, 58.0, 55.4, 50.1, 45.3, 43.8, 43.0, 28.2. IR 3344 (w), $2974(w)$, 2936 (w), 1727 (m), 1654 (m), 1596 (m), 1519 (m), 1457 (m), 1429 (w), 1394 (w), 1365 (w), 1350 (m), 1294 (w), 1205 (m), 1153 (s), 1064 (m), 960 (w), 839 (w), 781 (w), 754 (w), 735 (w), $700(\mathrm{~m})$.

HRMS (nanochip-ESI/LTQ-Orbitrap): Calcd for $\mathrm{C}_{27} \mathrm{H}_{34} \mathrm{NO}_{5}{ }^{+}[\mathrm{M}+\mathrm{H}]^{+} 452.2431$; Found 452.2441 .

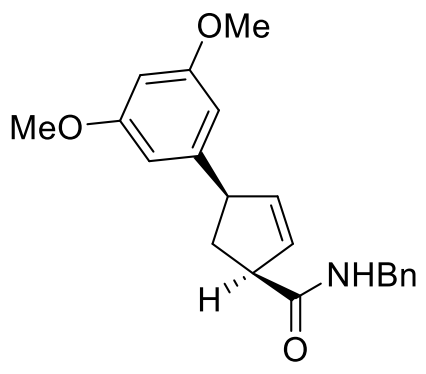

$3 \mathbf{k g}$

According to the general procedure, $3 \mathbf{k g}$ was obtained in $63 \%$ yield $(21.3 \mathrm{mg})$ as colorless oil with 5:1 dr and 96\% ee which was measured by SFC on Chiralcel IB (5\% MeOH in supercritical $\mathrm{CO}_{2}$ as eluent; flow rate $4.0 \mathrm{~mL} / \mathrm{min}$; $\mathrm{tR}$ (major) $=20.3 \mathrm{~min} ., \mathrm{tR}($ minor $)=17.1 \mathrm{~min}$.). $[\alpha]_{D}^{25}$ $+300.8\left(\right.$ c 0.97, $\left.\mathrm{CHCl}_{3}\right)$.

${ }^{1} \mathbf{H}$ NMR $\left(400 \mathrm{MHz}, \mathrm{CDCl}_{3}\right) \delta$ 7.34-7.22 (m, 5H), $6.38(\mathrm{~d}, J=2.3 \mathrm{~Hz}, 2 \mathrm{H}), 6.31(\mathrm{t}, J=2.3 \mathrm{~Hz}$, 
$1 \mathrm{H}), 5.98(\mathrm{dt}, J=5.7,2.2 \mathrm{~Hz}, 1 \mathrm{H}), 5.91(\mathrm{dt}, J=5.7,2.2 \mathrm{~Hz}, 1 \mathrm{H}), 5.78(\mathrm{t}, J=8.0 \mathrm{~Hz}, 1 \mathrm{H}), 4.44$ $(\mathrm{d}, J=5.6 \mathrm{~Hz}, 2 \mathrm{H}), 3.93-3.87(\mathrm{~m}, 1 \mathrm{H}), 3.75(\mathrm{~s}, 6 \mathrm{H}), 3.58-3.52(\mathrm{~m}, 1 \mathrm{H}), 2.78(\mathrm{dt}, J=13.1,8.8$ $\mathrm{Hz}, 1 \mathrm{H}), 1.96$ (dt, $J=13.1,7.6 \mathrm{~Hz}, 1 \mathrm{H}) ;{ }^{13} \mathbf{C}$ NMR (101 MHz, $\left.\mathrm{CDCl}_{3}\right) \delta 174.1,161.1,147.4$, 138.34, 138.25, 130.3, 128.9, 127.9, 127.7, 105.5, 98.5, 55.4, 52.8, 51.4, 43.8, 38.1. IR 3327 (m), 3312 (m), 3296 (m), 3269 (m), 3059 (m), 2837 (w), 2361 (m), 2331 (m), 2159 (s), 1947 (w), 1801 (m), 1772 (w), 1650 (s), 1598 (s), 1540 (m), 1523 (m), 1459 (m), 1430 (m), 1348 (m), $1326(\mathrm{w}), 1294(\mathrm{w}), 1205(\mathrm{~m}), 1155(\mathrm{~s}), 1010(\mathrm{w}), 991(\mathrm{w}), 976(\mathrm{w}), 937(\mathrm{w}), 868(\mathrm{w}), 835(\mathrm{w})$, $796(\mathrm{w}), 771(\mathrm{w}), 754(\mathrm{w}), 723(\mathrm{~m}), 710(\mathrm{w}), 681(\mathrm{w}), 661(\mathrm{w})$.

HRMS (ESI/QTOF): Calcd for $\mathrm{C}_{21} \mathrm{H}_{23} \mathrm{NNaO}_{3}{ }^{+}[\mathrm{M}+\mathrm{Na}]^{+}$360.1570; Found 360.1572.

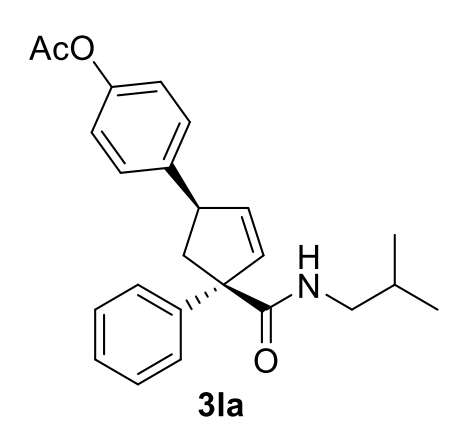

According to the general procedure, 3la was obtained in $66 \%$ yield $(25.0 \mathrm{mg})$ as colorless oil with 10:1 dr and $82 \%$ ee which was measured by SFC on Chiralcel AD-H (10\% $\mathrm{MeOH}$ in supercritical $\mathrm{CO}_{2}$ as eluent; flow rate $4.0 \mathrm{~mL} / \mathrm{min}$; $\mathrm{tR}$ (major) $=7.9 \mathrm{~min}$., $\mathrm{tR}$ (minor) $=5.6 \mathrm{~min}$.). $[\alpha]_{D}^{25}+70.5\left(c 0.76, \mathrm{CHCl}_{3}\right)$.

${ }^{1} \mathbf{H}$ NMR $\left(400 \mathrm{MHz}, \mathrm{CDCl}_{3}\right) \delta$ 7.37-7.36 (m, 4H), 7.31-7.25 (m, 3H), 7.02-6.99 (m, 2H), 6.17 $(\mathrm{dd}, J=5.5,2.6 \mathrm{~Hz}, 1 \mathrm{H}), 6.01(\mathrm{dd}, J=5.5,1.9 \mathrm{~Hz}, 1 \mathrm{H}), 5.34(\mathrm{t}, J=5.5 \mathrm{~Hz}, 1 \mathrm{H}), 3.91(\mathrm{tt}, J=$ 8.0, 2.2 Hz, 1H), 3.10-2.98 (m, 2H), 2.89 (dd, $J=13.3,8.1 \mathrm{~Hz}, 1 \mathrm{H}), 2.68(\mathrm{dd}, J=13.3,8.0 \mathrm{~Hz}$, $1 \mathrm{H}), 2.29(\mathrm{~s}, 3 \mathrm{H}), 1.74-1.64(\mathrm{~m}, 1 \mathrm{H}), 0.80(\mathrm{~d}, J=6.7 \mathrm{~Hz}, 3 \mathrm{H}), 0.79(\mathrm{~d}, J=6.7 \mathrm{~Hz}, 3 \mathrm{H}) ;{ }^{13} \mathrm{C}$ NMR $\left(101 \mathrm{MHz}, \mathrm{CDCl}_{3}\right) \delta 174.9,169.8,149.3,143.9,142.0,137.5,134.4,128.9,128.7,127.3$, 126.9, 121.6, 65.7, 50.0, 47.4, 45.8, 28.6, 21.3, 20.1. IR 2959 (s), 2527 (s), 2358 (s), 1752 (s), 1668 (s), 1650 (s), 1540 (s), 1523 (s), 1509 (s), 1371 (m), 1197 (s), 1039 (w), 1014 (w), 908 (w), $760(\mathrm{w}), 702(\mathrm{w}), 660(\mathrm{w})$.

HRMS (ESI/QTOF) : Calcd for $\mathrm{C}_{24} \mathrm{H}_{28} \mathrm{NO}_{3}{ }^{+}[\mathrm{M}+\mathrm{H}]^{+}$378.2064; Found 378.2061. 


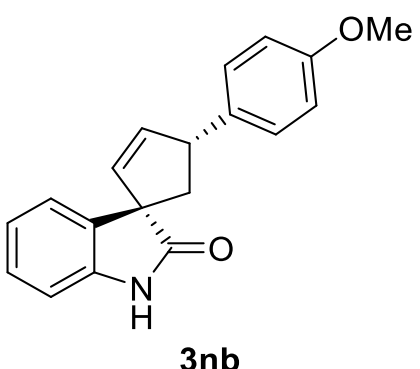

According to the general procedure, 3nb was obtained in $88 \%$ yield $(25.5 \mathrm{mg})$ as colorless solid (mp 126-128 ${ }^{\circ} \mathrm{C}$ ) with >19:1 dr and 79\% ee which was measured by SFC on Chiralcel IC $(10 \%$ $\mathrm{MeOH}$ in supercritical $\mathrm{CO}_{2}$ as eluent; flow rate $4.0 \mathrm{~mL} / \mathrm{min}$; $\mathrm{tR}$ (major) $=9.3 \mathrm{~min}$., $\mathrm{tR}$ (minor) $=7.9$ min. $) .[\alpha]_{D}^{25}+278.8\left(c 1.10, \mathrm{CHCl}_{3}\right)$.

${ }^{1} \mathbf{H}$ NMR $\left(400 \mathrm{MHz}, \mathrm{CDCl}_{3}\right) \delta 8.11$ (brs, 1H), 7.35-7.31 (m, 2H), 7.24-7.19 (m, 2H), 7.04 (dt, $J=7.5,0.9 \mathrm{~Hz}, 1 \mathrm{H}), 6.91-6.88(\mathrm{~m}, 3 \mathrm{H}), 6.12(\mathrm{dd}, J=5.4,1.9 \mathrm{~Hz}, 1 \mathrm{H}), 5.65(\mathrm{dd}, J=5.4,2.5$ $\mathrm{Hz}, 1 \mathrm{H}), 4.33(\mathrm{tt}, J=8.1,2.1 \mathrm{~Hz}, 1 \mathrm{H}), 3.81(\mathrm{~s}, 3 \mathrm{H}), 2.60(\mathrm{dd}, J=13.2,8.2 \mathrm{~Hz}, 1 \mathrm{H}), 2.43(\mathrm{dd}, J$ $=13.2,8.1 \mathrm{~Hz}, 1 \mathrm{H}) ;{ }^{13} \mathbf{C}$ NMR $\left(101 \mathrm{MHz}, \mathrm{CDCl}_{3}\right) \delta 181.7,158.6,140.0,139.5,136.5,134.4$, 132.0, 128.8, 128.3, 123.6, 122.9, 114.1, 109.8, 61.8, 55.5, 51.1, 45.9. IR $3287(\mathrm{w}), 3265$ (w), 3233 (w), 3217 (w), 3215 (w), 3190 (w), 3179 (w), 2361 (m), 2340 (m), $2022(\mathrm{w}), 1770(\mathrm{w})$, 1710 (s), 1654 (w), 1617 (m), 1513 (m), 1471 (m), 1446 (w), 1332 (w), 1303 (w), 1109 (w), $1035(\mathrm{w}), 1010(\mathrm{w}), 1008(\mathrm{w}), 831(\mathrm{w}), 829(\mathrm{w}), 756(\mathrm{~m}), 708(\mathrm{w}), 687(\mathrm{w}), 660(\mathrm{w})$.

HRMS (ESI/QTOF) : Calcd for $\mathrm{C}_{19} \mathrm{H}_{17} \mathrm{NNaO}_{2}{ }^{+}[\mathrm{M}+\mathrm{Na}]^{+}$314.1151; Found 314.1155.

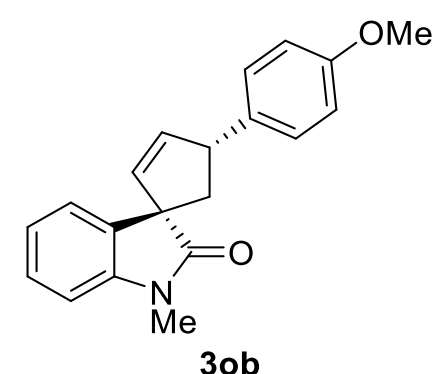

According to the general procedure, $\mathbf{3 o b}^{3}$ was obtained in $85 \%$ yield $(25.9 \mathrm{mg})$ as colorless oil with >19:1 dr and 79\% ee which was measured by SFC on Chiralcel IA (10\% $\mathrm{MeOH}$ in supercritical $\mathrm{CO}_{2}$ as eluent; flow rate $4.0 \mathrm{~mL} / \mathrm{min}$; $\mathrm{tR}$ (major) $=5.1 \mathrm{~min}$., $\mathrm{tR}$ (minor) = $4.6 \mathrm{~min}$.). $[\alpha]_{D}^{25}+175.1\left(c 1.30, \mathrm{CHCl}_{3}\right)$.

${ }^{1} \mathbf{H}$ NMR $\left(400 \mathrm{MHz}, \mathrm{CDCl}_{3}\right) \delta 7.36(\mathrm{~d}, J=8.3 \mathrm{~Hz}, 2 \mathrm{H}), 7.28(\mathrm{t}, J=7.6 \mathrm{~Hz}, 1 \mathrm{H}), 7.21(\mathrm{~d}, J=$ $7.2 \mathrm{~Hz}, 1 \mathrm{H}), 7.06$ (t, $J=7.4 \mathrm{~Hz}, 1 \mathrm{H}), 6.89$ (d, $J=8.3 \mathrm{~Hz}, 2 \mathrm{H}), 6.84(\mathrm{~d}, J=7.7 \mathrm{~Hz}, 1 \mathrm{H}), 6.09$ (d, $J=4.3 \mathrm{~Hz}, 1 \mathrm{H}), 5.57(\mathrm{~d}, J=4.7 \mathrm{~Hz}, 1 \mathrm{H}), 4.32(\mathrm{t}, J=7.7 \mathrm{~Hz}, 1 \mathrm{H}), 3.80(\mathrm{~s}, 3 \mathrm{H}), 3.24(\mathrm{~s}, 3 \mathrm{H})$, $2.56(\mathrm{dd}, J=13.2,8.4 \mathrm{~Hz}, 1 \mathrm{H}), 2.42(\mathrm{dd}, J=13.2,7.8 \mathrm{~Hz}, 1 \mathrm{H}) ;{ }^{13} \mathbf{C} \mathbf{N M R}\left(101 \mathrm{MHz}, \mathrm{CDCl}_{3}\right) \delta$ 
179.7, 158.5, 143.0, 139.3, 136.6, 133.9, 132.1, 128.9, 128.3, 123.2, 122.9, 114.1, 108.1, 61.5, 55.4, 51.2, 45.7, 26.6. IR 2936 (m), 2361 (m), 2338 (m), 1710 (s), 1612 (s), 1513 (s), 1494 (m), 1471 (m), 1423 (w), 1375 (m), 1350 (m), 1305 (w), 1253 (m), 1180 (w), 1134 (w), 1124 (w), $1091(\mathrm{w}), 1035(\mathrm{w}), 1012(\mathrm{w}), 935(\mathrm{w}), 831(\mathrm{w}), 769(\mathrm{w}), 752(\mathrm{w}), 723(\mathrm{w}), 690(\mathrm{w})$.

HRMS (ESI/QTOF) : Calcd for $\mathrm{C}_{20} \mathrm{H}_{19} \mathrm{NNaO}_{2}{ }^{+}[\mathrm{M}+\mathrm{Na}]^{+}$328.1308; Found 328.1307.

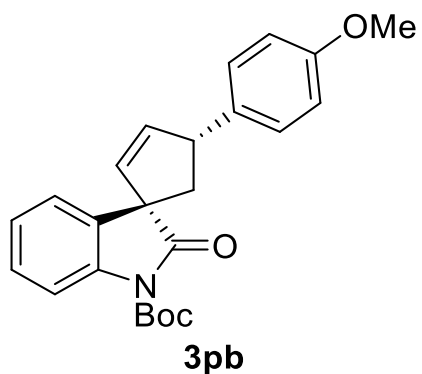

According to the general procedure, $\mathbf{3} \mathbf{p b}^{3}$ was obtained in $77 \%$ yield $(30.0 \mathrm{mg})$ as white solid (mp 107-108 ${ }^{\circ} \mathrm{C}$ ) with $15: 1 \mathrm{dr}$ and $75 \%$ ee which was measured by SFC on Chiralcel IC (5\% $\mathrm{MeOH}$ in supercritical $\mathrm{CO}_{2}$ as eluent; flow rate $4.0 \mathrm{~mL} / \mathrm{min}$; $\mathrm{tR}$ (major) $=8.9 \mathrm{~min}$., $\mathrm{tR}$ (minor) $=7.7$ min. $)$. $[\alpha]_{D}^{25}+153.4\left(\right.$ c $\left.1.43, \mathrm{CHCl}_{3}\right)$.

${ }^{1} \mathbf{H}$ NMR $\left(400 \mathrm{MHz}, \mathrm{CDCl}_{3}\right) \delta 7.86(\mathrm{~d}, J=8.1 \mathrm{~Hz}, 1 \mathrm{H}), 7.33-7.28(\mathrm{~m}, 3 \mathrm{H}), 7.22-7.15(\mathrm{~m}, 2 \mathrm{H})$, 6.89-6.87 (m, 2H), $6.08(\mathrm{dd}, J=5.3,1.9 \mathrm{~Hz}, 1 \mathrm{H}), 5.63(\mathrm{dd}, J=5.3,2.5 \mathrm{~Hz}, 1 \mathrm{H}), 4.31$ (tt, $J=$ 8.0, 2.0 Hz, 1H), 3.80 (s, 3H), 2.61 (dd, $J=13.4,8.4 \mathrm{~Hz}, 1 \mathrm{H}), 2.47$ (dd, $J=13.4,7.8 \mathrm{~Hz}, 1 \mathrm{H})$, 1.65 (s, 9H); ${ }^{13} \mathrm{C}$ NMR (101 MHz, $\left.\mathrm{CDCl}_{3}\right) \delta$ 178.3, 158.6, 149.5, 139.4, 138.7, 136.2, 132.7, 132.1, 128.8, 128.4, 124.8, 123.2, 115.1, 114.1, 84.4, 61.9, 55.4, 51.1, 47.0, 28.2. IR 2978 (w), 2938 (w), 2361 (w), 2161 (w), 1793 (m), 1766 (s), 1731 (s), 1610 (w), 1513 (m), 1477 (m), 1465 (m), 1371 (w), 1350 (m), 1294 (s), 1251 (s), 1151 (s), 1084 (w), 1037 (w), 1008 (w), 837 (w), $769(\mathrm{w}), 754(\mathrm{w}), 727(\mathrm{w}), 698(\mathrm{w}), 661(\mathrm{w})$.

HRMS (nanochip-ESI/LTQ-Orbitrap): Calcd for $\mathrm{C}_{24} \mathrm{H}_{25} \mathrm{NNaO}_{4}{ }^{+}[\mathrm{M}+\mathrm{Na}]^{+}$414.1676; Found 414.1688 . 


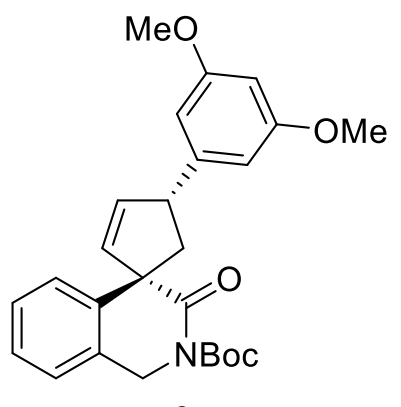

$3 q g$

According to the general procedure, 3qg was obtained in $61 \%$ yield $(26.5 \mathrm{mg}$ ) as colorless oil with $15: 1 \mathrm{dr}$ and $92 \%$ ee which was measured by SFC on Chiralcel AD-H $(10 \% \mathrm{MeOH}$ in supercritical $\mathrm{CO}_{2}$ as eluent; flow rate $4.0 \mathrm{~mL} / \mathrm{min}$; $\mathrm{tR}$ (major) $=3.3 \mathrm{~min}$., $\mathrm{tR}$ (minor) $=5.8 \mathrm{~min}$.). $[\alpha]_{D}^{25}+103.3\left(c 0.75, \mathrm{CHCl}_{3}\right)$.

${ }^{1} \mathbf{H}$ NMR $\left(400 \mathrm{MHz}, \mathrm{CDCl}_{3}\right) \delta$ 7.32-7.30 (m, 3H), 7.26-7.24 (m, 1H), $6.40(\mathrm{~d}, J=2.2 \mathrm{~Hz}, 2 \mathrm{H})$, $6.32(\mathrm{t}, J=2.2 \mathrm{~Hz}, 1 \mathrm{H}), 6.23(\mathrm{dd}, J=5.6,1.7 \mathrm{~Hz}, 1 \mathrm{H}), 6.10(\mathrm{dd}, J=5.6,2.6 \mathrm{~Hz}, 1 \mathrm{H}), 5.12(\mathrm{~d}$, $J=15.8 \mathrm{~Hz}, 1 \mathrm{H}), 4.65(\mathrm{~d}, J=15.8 \mathrm{~Hz}, 1 \mathrm{H}), 3.90(\mathrm{t}, J=7.8 \mathrm{~Hz}, 1 \mathrm{H}), 3.77(\mathrm{~s}, 6 \mathrm{H}), 1 \mathrm{H}), 2.51$ $(\mathrm{dd}, J=12.6,7.8 \mathrm{~Hz}, 1 \mathrm{H}), 2.35(\mathrm{dd}, J=12.6,8.0 \mathrm{~Hz}, 1 \mathrm{H}), 1.55(\mathrm{~s}, 9 \mathrm{H}) ;{ }^{13} \mathrm{C}$ NMR $(101 \mathrm{MHz}$, $\left.\mathrm{CDCl}_{3}\right) \delta 173.6,161.1,152.7,146.4,139.3,137.8,132.6,131.9,128.3,127.4,126.5,124.6$, 105.5, 99.0, 83.7, 63.3, 55.5, 50.7, 47.7, 46.1, 28.2. IR 2976 (w), 2940 (w), 2897 (w), $2882(w)$, 2872 (w), 2839 (w), 2159 (w), 1772 (w), 1718 (m), 1598 (m), 1463 (w), 1430 (w), 1388 (w), 1371 (w), 1353 (w), 1299 (m), 1263 (w), 1224 (w), 1205 (m), 1153 (s), 1066 (w), 997 (w), 926 (w), $856(\mathrm{w}), 854(\mathrm{w}), 771(\mathrm{w}), 752(\mathrm{w}), 696(\mathrm{w})$.

HRMS (ESI/QTOF): Calcd for $\mathrm{C}_{26} \mathrm{H}_{29} \mathrm{NNaO}_{5}{ }^{+}[\mathrm{M}+\mathrm{Na}]^{+} 458.1938$; Found 458.1948.

\section{Scale up reactions and post-transformations}

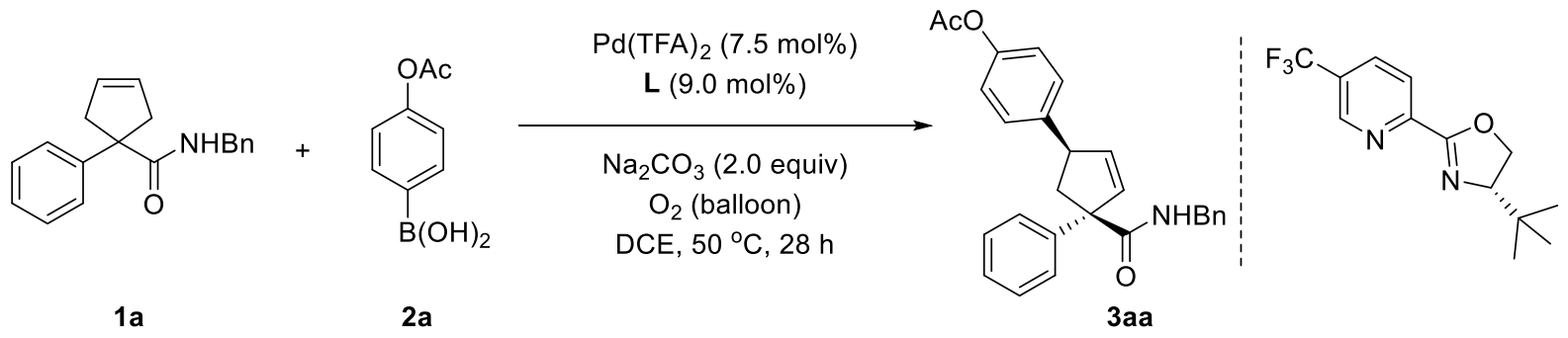

A $100 \mathrm{~mL}$ round-bottom flask were charged with $\mathrm{Pd}(\mathrm{TFA})_{2}(25.0 \mathrm{mg}, 0.075 \mathrm{mmol})$, ligand (25.0 $\mathrm{mg}, 0.09 \mathrm{mmol})$ and DCE $(8 \mathrm{~mL})$, then the mixture was stirred at RT for about $1 \mathrm{~h}$. 1a (277.4 mg, $1.0 \mathrm{mmol}), \mathbf{2 a}(360.0 \mathrm{mg}, 2.0 \mathrm{mmol}), \mathrm{Na}_{2} \mathrm{CO}_{3}(212.0 \mathrm{mg}, 2.0 \mathrm{mmol})$ and additional DCE $(12 \mathrm{~mL})$ were added sequentially. After purging with $\mathrm{O}_{2}$, the mixture was stirred at $50{ }^{\circ} \mathrm{C}$ with an $\mathrm{O}_{2}$ balloon for $28 \mathrm{hrs}$. After cooling to RT, the mixture was diluted with $\mathrm{CH}_{2} \mathrm{Cl}_{2}$ and washed with water, $1 \mathrm{M} \mathrm{HCl}$ solution, brine and dried over $\mathrm{Na}_{2} \mathrm{SO}_{4}$. After concentrating, the 
residue was subjected to ${ }^{1} \mathrm{H}$ NMR for $\mathrm{dr}$ value analysis and then was purified by flash chromatography (silica gel, eluted with petroleum ether/ethyl acetate) to give product 3aa (284.8 mg, 69\% yield) with >19:1 dr and 93\% ee. $89.3 \mathrm{mg}$ 1a was recovered.
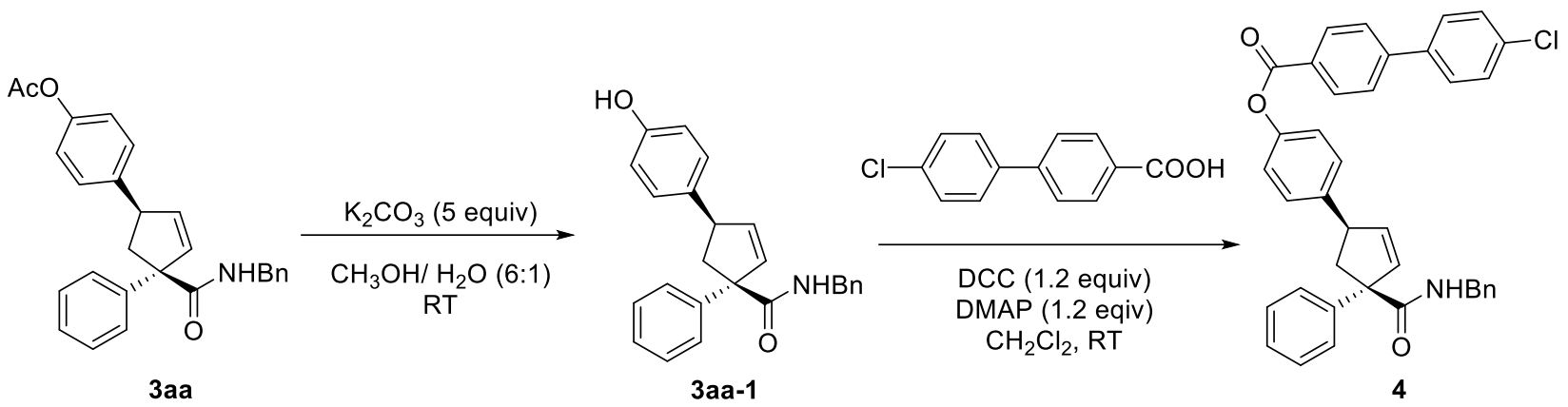

To a solution of 3aa $(33.6 \mathrm{mg}, 0.08 \mathrm{mmol})$ in $\mathrm{CH}_{3} \mathrm{OH}-\mathrm{H}_{2} \mathrm{O}(6: 1,3.5 \mathrm{~mL})$ was added $\mathrm{K}_{2} \mathrm{CO}_{3}(57$ $\mathrm{mg}, 0.41 \mathrm{mmol}$ ), then the mixture was stirred at RT for $30 \mathrm{~min}$. After cooling to $0{ }^{\circ} \mathrm{C}, 0.5 \mathrm{M}$ $\mathrm{HCl}$ solution was added dropwise to quench the reaction, then the mixture was extracted with $\mathrm{CH}_{2} \mathrm{Cl}_{2}$. The combined organic extracts were dried over $\mathrm{Na}_{2} \mathrm{SO}_{4}$. After concentrating, the residue was purified by flash chromatography (silica gel, eluted with petroleum ether/ethyl acetate) to give product 3aa-1 $(29.7 \mathrm{mg}, 98 \%$ yield $)$ as colorless oil. $[\alpha]_{D}^{25}+160.3(c 1.93$, $\left.\mathrm{CHCl}_{3}\right)$.

${ }^{1} \mathbf{H}$ NMR $\left(400 \mathrm{MHz}, \mathrm{CDCl}_{3}\right) \delta 7.60(\mathrm{~s}, 1 \mathrm{H}), 7.37-7.32(\mathrm{~m}, 4 \mathrm{H}), 7.28-7.19(\mathrm{~m}, 4 \mathrm{H}), 7.11(\mathrm{~d}, J$ $=6.6 \mathrm{~Hz}, 2 \mathrm{H}), 7.04(\mathrm{~d}, J=8.5 \mathrm{~Hz}, 2 \mathrm{H}), 6.79(\mathrm{~d}, J=8.5 \mathrm{~Hz}, 2 \mathrm{H}), 6.15(\mathrm{dd}, J=5.5,2.5 \mathrm{~Hz}, 1 \mathrm{H})$, $6.04(\mathrm{dd}, J=5.5,1.8 \mathrm{~Hz}, 1 \mathrm{H}), 5.79$ (t, $J=5.4 \mathrm{~Hz}, 1 \mathrm{H}), 4.43(\mathrm{dd}, J=15.0,5.8 \mathrm{~Hz}, 1 \mathrm{H}), 4.38$ $(\mathrm{dd}, J=15.0,5.7 \mathrm{~Hz}, 1 \mathrm{H}) 3.85(\mathrm{tt}, J=7.8,2.4 \mathrm{~Hz}, 1 \mathrm{H}), 2.88(\mathrm{dd}, J=13.3,7.8 \mathrm{~Hz}, 1 \mathrm{H}), 2.69$ $(\mathrm{dd}, J=13.3,8.0 \mathrm{~Hz}, 1 \mathrm{H}) ;{ }^{13} \mathbf{C}$ NMR $\left(101 \mathrm{MHz}, \mathrm{CDCl}_{3}\right) \delta 176.1,155.5,143.4,138.6,137.9$, 135.2, 133.3, 129.0, 128.8, 128.6, 127.6, 127.5, 126.9, 115.7, 65.4, 49.7, 46.2, 44.2. IR 3356 (w), $3340(\mathrm{w}), 3314$ (w), 3267 (w), 3024 (w), 2165 (w), 1646 (m), 1615 (w), $1596(\mathrm{w}), 1513$ (s), $1450(\mathrm{~m}), 1359(\mathrm{w}), 1224(\mathrm{~m}), 1172(\mathrm{w}), 1101(\mathrm{w}), 1078(\mathrm{w}), 1060(\mathrm{w}), 1008(\mathrm{w}), 970(\mathrm{w})$, $954(\mathrm{w}), 937$ (w), 914 (w), $900(\mathrm{w}), 835$ (m), 798 (w), 754 (s), 698 (s), 677 (m), 663 (m).

HRMS (ESI/QTOF): Calcd for $\mathrm{C}_{25} \mathrm{H}_{23} \mathrm{NNaO}_{2}{ }^{+}[\mathrm{M}+\mathrm{Na}]^{+} 392.1621$; Found 392.1615.

The solution of 3aa-1 (22.0 mg, $0.06 \mathrm{mmg}$ ), 4'-chloro-[1,1'-biphenyl]-4-carboxylic acid (16.8 $\mathrm{mg}, 0.072 \mathrm{mmol}$ ), DCC (15.0 mg, $0.072 \mathrm{mmol})$ and DMAP (9.0 mg, $0.072 \mathrm{mmol})$ in $\mathrm{CH}_{2} \mathrm{Cl}_{2}$ (2 $\mathrm{mL})$ was stirred overnight. After concentrating, the residue was purified via flash chromatography (silica gel, eluted with petroleum ether/ethyl acetate) to give product 4 (34.5 
mg, 99\% yield) as white solid. $[\alpha]_{D}^{25}+160.0$ (c 2.05, $\left.\mathrm{CHCl}_{3}\right), \mathrm{mp} 251-253{ }^{\circ} \mathrm{C}$ (decomposed). ${ }^{1}$ H NMR (400 MHz, $\left.\mathrm{CDCl}_{3}\right) \delta$ 8.28-8.25 (m, 2H), 7.71-7.68 (m, 2H), 7.61-7.57 (m, 2H), 7.48$7.44(\mathrm{~m}, 2 \mathrm{H}), 7.39-7.24(\mathrm{~m}, 10 \mathrm{H}), 7.18-7.14(\mathrm{~m}, 4 \mathrm{H}), 6.21(\mathrm{dd}, J=5.6,2.6 \mathrm{~Hz}, 1 \mathrm{H}), 6.07$ (dd, $J=5.5,1.9 \mathrm{~Hz}, 1 \mathrm{H}), 5.64(\mathrm{t}, J=5.3 \mathrm{~Hz}, 1 \mathrm{H}), 4.47(\mathrm{dd}, J=14.9,5.8 \mathrm{~Hz}, 1 \mathrm{H}), 4.41(\mathrm{dd}, J=14.9$, $5.8 \mathrm{~Hz}, 1 \mathrm{H}), 3.97(\mathrm{tt}, J=7.9,2.1 \mathrm{~Hz}, 1 \mathrm{H}), 2.95(\mathrm{dd}, J=13.3,8.0 \mathrm{~Hz}, 1 \mathrm{H}), 2.74(\mathrm{dd}, J=13.3$, $8.0 \mathrm{~Hz}, 1 \mathrm{H}) ;{ }^{13} \mathrm{C}$ NMR $\left(101 \mathrm{MHz}, \mathrm{CDCl}_{3}\right) \delta$ 174.9, 165.2, 149.6, 145.1, 143.6, 142.0, 138.5, $138.4,137.7,134.7,134.2$, 131.0, 129.3, 129.0, 128.8, 128.7, 127.63, 127.55, 127.4, 127.2, 126.9, 121.8, 65.6, 50.1, 46.0, 44.1. IR 3071 (w), 3069 (w), 2928 (w), 2363 (m), 2167 (m), 2049 (w), 1978 (w), 1735 (s), 1652 (m), 1608 (w), 1529 (m), 1527 (m), 1506 (m), 1271 (s), 1269 (s), 1197 (s), 1172 (m), 1170 (m), 1099 (w), 1078 (m), 1018 (w), 1016 (w), 1006 (w), 899 (w), $858(\mathrm{w}), 846(\mathrm{w}), 821(\mathrm{~m}), 798(\mathrm{w}), 767$ (m), $758(\mathrm{~m}), 727$ (s), $694(\mathrm{~m}), 673(\mathrm{~m}), 660(\mathrm{~s})$. HRMS (ESI/QTOF): Calcd for $\mathrm{C}_{38} \mathrm{H}_{30} \mathrm{ClNNaO}_{3}{ }^{+}[\mathrm{M}+\mathrm{Na}]^{+}$606.1806; Found 606.1810.
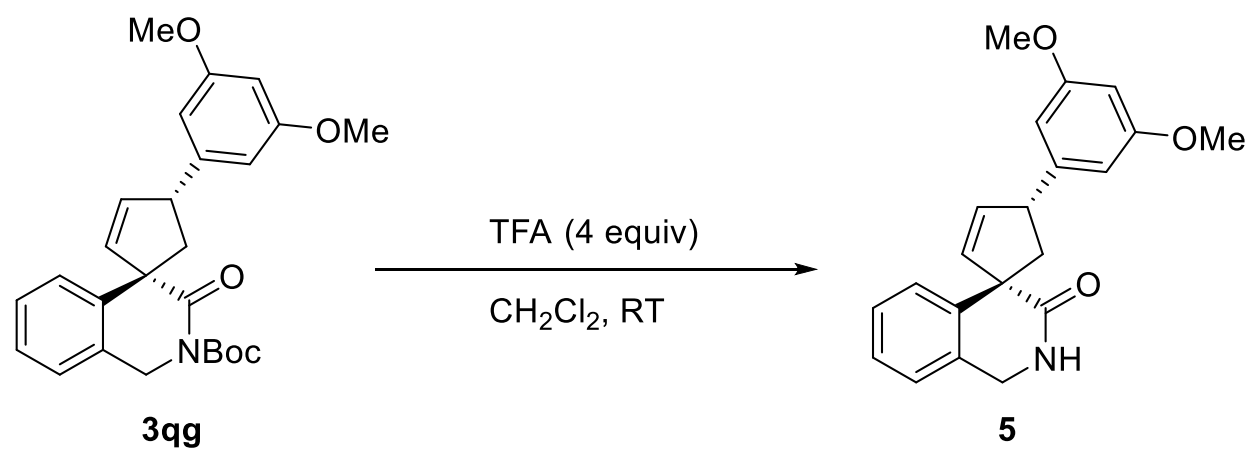

To a solution of $\mathbf{3 q g}(55.0 \mathrm{mg}, 0.126 \mathrm{mmol})$ in $\mathrm{CH}_{2} \mathrm{Cl}_{2}(10 \mathrm{~mL})$ was added trifluoroacetic acid (40 $\mathrm{uL}, 0.51 \mathrm{mmol}$ ), then the mixture was stirred at RT for $1.5 \mathrm{hrs}$. After cooling to $0{ }^{\circ} \mathrm{C}$, saturated $\mathrm{NaHCO}_{3}$ solution was added to quench the reaction. Layers were separated and the aqueous layer was extracted with $\mathrm{CH}_{2} \mathrm{Cl}_{2}$. The combined organic layers weer dried over $\mathrm{Na}_{2} \mathrm{SO}_{4}$. After concentrating, the residue was purified by flash chromatography (silica gel, eluted with petroleum ether/ethyl acetate) to give product 5 (33.4 mg, 79\% yield) as colorless solid. $[\alpha]_{D}^{25}$ $+261.2\left(\right.$ c $\left.1.09, \mathrm{CHCl}_{3}\right), \mathrm{mp} 156-157^{\circ} \mathrm{C}$.

${ }^{1} \mathbf{H}$ NMR $\left(400 \mathrm{MHz}, \mathrm{CDCl}_{3}\right) \delta$ 7.32-7.28 (m, 4H), $7.20(\mathrm{~d}, J=6.8 \mathrm{~Hz}, 1 \mathrm{H}), 6.48(\mathrm{~d}, J=2.1 \mathrm{~Hz}$, $2 \mathrm{H}), 6.32(\mathrm{t}, J=4.2 \mathrm{~Hz}, 1 \mathrm{H}), 6.21(\mathrm{dd}, J=5.5,1.3 \mathrm{~Hz}, 1 \mathrm{H}), 6.02(\mathrm{dd}, J=5.4,2.5 \mathrm{~Hz}, 1 \mathrm{H}), 4.60$ (d, $J=15.7 \mathrm{~Hz}, 1 \mathrm{H}), 4.40$ (dd, $J=15.8,4.3 \mathrm{~Hz}, 1 \mathrm{H}), 3.98-3.94(\mathrm{~m}, 1 \mathrm{H}), 3.77$ (s, 6H), 2.51 (dd, $J=12.7,7.9 \mathrm{~Hz}, 1 \mathrm{H}), 2.40(\mathrm{dd}, J=12.7,8.2 \mathrm{~Hz}, 1 \mathrm{H}) ;{ }^{13} \mathbf{C ~ N M R}\left(101 \mathrm{MHz}, \mathrm{CDCl}_{3}\right) \delta 176.6$, 161.0, 146.7, 139.3, 137.9, 133.2, 131.6, 127.8, 126.9, 125.9, 125.3, 105.7, 99.0, 59.9, 55.5, 50.9, 46.9, 44.8. IR 3206 (w), 3204 (w), 2959 (w), 2957 (w), 2938 (w), 2157 (w), 1669 (s), 1598 (s), 1463 (m), 1461 (m), 1430 (w), 1332 (w), 1330 (w), 1296 (w), 1205 (m), 1153 (s), 
$1062(\mathrm{w}), 1003(\mathrm{w}), 827(\mathrm{w}), 760(\mathrm{~m}), 723(\mathrm{w}), 694(\mathrm{w}), 675(\mathrm{w}), 661(\mathrm{w})$.

HRMS (nanochip-ESI/LTQ-Orbitrap): Calcd for $\mathrm{C}_{21} \mathrm{H}_{22} \mathrm{NO}_{3}{ }^{+}[\mathrm{M}+\mathrm{H}]^{+} 336.1594$; Found 336.1590 .

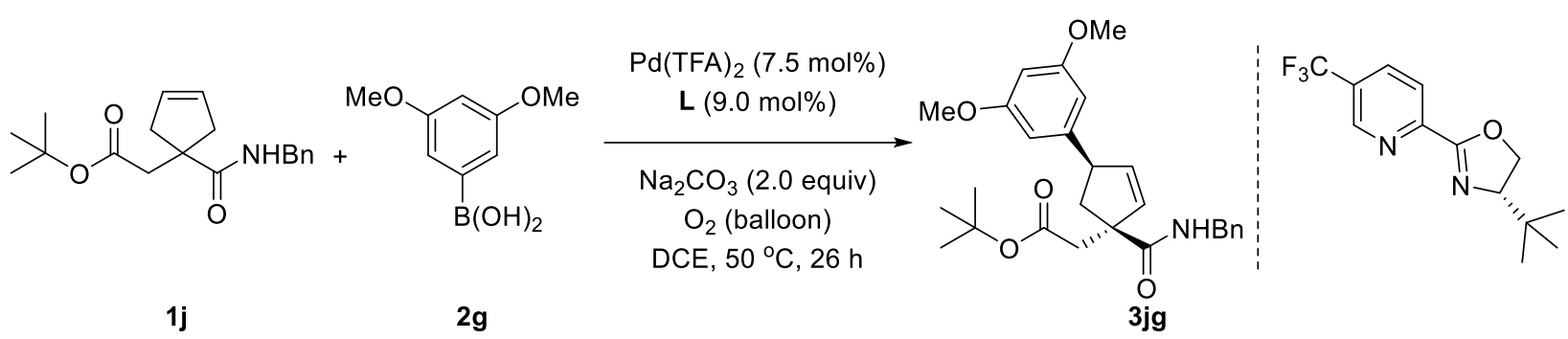

A mixture of Pd(TFA) $2(7.5 \mathrm{mg}, 0.0225 \mathrm{mmol})$ and the ligand $(7.5 \mathrm{mg}, 0.027 \mathrm{mmol})$ in DCE ( $2 \mathrm{~mL}$ ) was stirred at RT for $1 \mathrm{~h} . \mathbf{1 j}$ (94.7 mg, $0.3 \mathrm{mmol}), \mathbf{2 g}(112.0 \mathrm{mg}, 0.6 \mathrm{mmol}), \mathrm{Na}_{2} \mathrm{CO}_{3}$ $(63.6 \mathrm{mg}, 0.6 \mathrm{mmol})$ and additional DCE $(4 \mathrm{~mL})$ were added sequentially. After purging with $\mathrm{O}_{2}$, the mixture was stirred at $50{ }^{\circ} \mathrm{C}$ with an $\mathrm{O}_{2}$ balloon for $26 \mathrm{hrs}$. After cooling to RT, the mixture was diluted with $\mathrm{CH}_{2} \mathrm{Cl}_{2}$ and washed with water, $1 \mathrm{M} \mathrm{HCl}$ solution, brine and dried over anhydrous $\mathrm{Na}_{2} \mathrm{SO}_{4}$. After concentrating, the residue was subjected to ${ }^{1} \mathrm{H}$ NMR for dr value analysis and then was purified by flash chromatography (silica gel, eluted with petroleum ether/ethyl acetate) to give product $\mathbf{3 j g}$ (94.3 mg, 70\% yield) with >19:1 dr and 97\% ee.

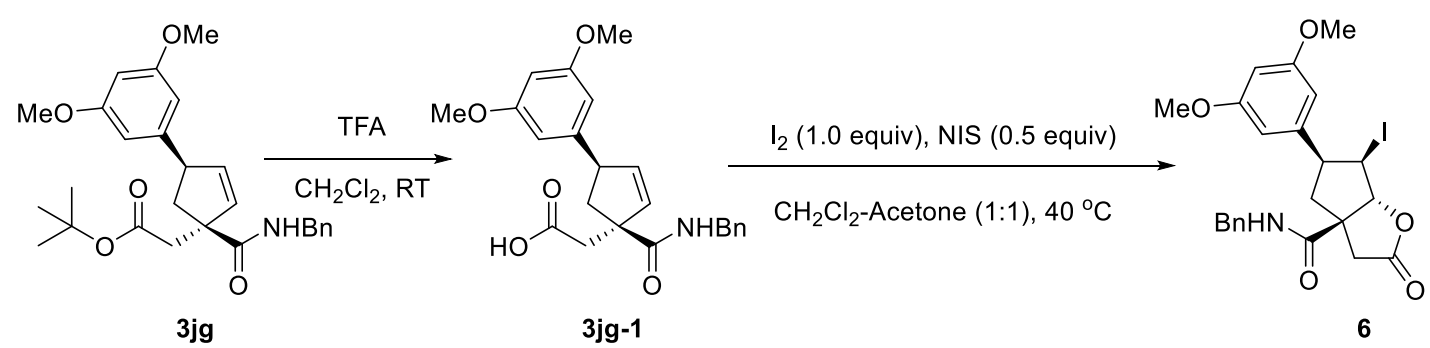

A solution of $3 \mathbf{j g}$ (21.0 mg, $0.046 \mathrm{mmol})$ in TFA $/ \mathrm{CH}_{2} \mathrm{Cl}_{2}(1.0 \mathrm{M}, 3.0 \mathrm{~mL})$ was stirred at RT for $2 \mathrm{hrs}$. Then the reaction mixture was concentrated and the residue was purified by flash chromatography (silica gel, eluted with petroleum ether/ethyl acetate) to give product $\mathbf{3 j g}-\mathbf{1}$ (18.0 mg, 98\% yield) as colorless oil. $[\alpha]_{D}^{25}+299.8\left(\right.$ c 1.01, $\left.\mathrm{CHCl}_{3}\right)$.

${ }^{1} \mathbf{H}$ NMR $\left(400 \mathrm{MHz}, \mathrm{CDCl}_{3}\right) \delta$ 7.31-7.25 (m, 4H), 7.18-7.16 (m, 2H), $6.30(\mathrm{t}, J=2.2 \mathrm{~Hz}, 1 \mathrm{H})$, $6.26(\mathrm{~d}, J=2.2 \mathrm{~Hz}, 2 \mathrm{H}), 6.16(\mathrm{t}, J=5.5 \mathrm{~Hz}, 1 \mathrm{H}), 6.12(\mathrm{dd}, J=5.5,1.9 \mathrm{~Hz}, 1 \mathrm{H}), 6.03(\mathrm{dd}, J=$ $5.3,1.7 \mathrm{~Hz}, 1 \mathrm{H}), 4.43(\mathrm{dd}, J=15.4,5.6 \mathrm{~Hz}, 1 \mathrm{H}), 4.39(\mathrm{dd}, J=15.2,5.8 \mathrm{~Hz}, 1 \mathrm{H}), 4.04(\mathrm{t}, J=$ $8.2 \mathrm{~Hz}, 1 \mathrm{H}), 3.72(\mathrm{~s}, 6 \mathrm{H}), 3.05(\mathrm{~d}, J=16.0 \mathrm{~Hz}, 1 \mathrm{H}), 2.77$ (d, $J=16.0 \mathrm{~Hz}, 1 \mathrm{H}), 2.62$ (dd, $J=$ 13.9, 8.7 Hz, 1H), 2.09 (dd, $J=13.9,7.0 \mathrm{~Hz}, 1 \mathrm{H}) ;{ }^{13} \mathbf{C ~ N M R}\left(101 \mathrm{MHz}, \mathrm{CDCl}_{3}\right) \delta 176.9,174.8$, 161.2, 146.2, 139.6, 137.6, 133.5, 128.9, 127.71, 127.65, 105.2, 98.5, 57.4, 55.4, 50.1, 45.0, 44.1, 42.4. IR 3063 (w), 3061 (w), 2934 (w), 2365 (w), 2363 (w), 2165 (w), 1974 (w), 1725 
(m), 1654 (m), 1652 (m), 1598 (s), 1531 (m), 1529 (m), 1502 (w), 1459 (m), 1430 (m), 1357 (w), $1344(\mathrm{w}), 1328(\mathrm{w}), 1205(\mathrm{~s}), 1155$ (s), 1062 (w), 993 (w), $933(\mathrm{w}), 845(\mathrm{w}), 829(\mathrm{w}), 798$ (w), $771(\mathrm{w}), 758(\mathrm{w}), 731(\mathrm{w}), 692(\mathrm{~m}), 677(\mathrm{w}), 660(\mathrm{~m})$.

HRMS (ESI/QTOF): Calcd for $\mathrm{C}_{23} \mathrm{H}_{25} \mathrm{NNaO}_{5}^{+}[\mathrm{M}+\mathrm{Na}]^{+} 418.1625$; Found 418.1617.

To a solution of 3jg-1 $(8.4 \mathrm{mg}, 0.021 \mathrm{mmol})$ in acetone- $\mathrm{CH}_{2} \mathrm{Cl}_{2}(1: 1,1.0 \mathrm{~mL})$ were added NIS (4.7 $\mathrm{mg}, 0.021 \mathrm{mmol})$ and iodine $(2.6 \mathrm{mg}, 0.01 \mathrm{mmol})$ sequentially. Then the mixture was stirred in dark at $40{ }^{\circ} \mathrm{C}$ for $15 \mathrm{hrs}$. After cooling to RT, saturated $\mathrm{Na}_{2} \mathrm{~S}_{2} \mathrm{O}_{3}$ solution was added. The reaction mixture was concentrated and the residue was purified by flash chromatography (silica gel, eluted with petroleum ether/ethyl acetate) to give product $6(7.3 \mathrm{mg}, 66 \%$ yield) as white wax. $[\alpha]_{D}^{25}+721.2\left(c 0.32, \mathrm{CHCl}_{3}\right)$.

${ }^{1}$ H NMR (400 MHz, $\left.\mathrm{CDCl}_{3}\right) \delta$ 7.39-7.29 (m, 5H), 6.44-6.41 (m, 1H), 6.39 (t, $\left.J=2.1 \mathrm{~Hz}, 1 \mathrm{H}\right)$, $6.25(\mathrm{~d}, J=1.9 \mathrm{~Hz}, 2 \mathrm{H}), 5.68(\mathrm{~s}, 1 \mathrm{H}), 4.69$ (dd, $J=4.5,1.2 \mathrm{~Hz}, 1 \mathrm{H}), 4.61-4.49$ (m, 2H), 3.78 (s, 6H), $3.60(\mathrm{~d}, J=19.0 \mathrm{~Hz}, 1 \mathrm{H}), 2.95-2.90(\mathrm{~m}, 1 \mathrm{H}), 2.75(\mathrm{t}, J=12.7 \mathrm{~Hz}, 1 \mathrm{H}), 2.63(\mathrm{~d}, J=$ $19.0 \mathrm{~Hz}, 1 \mathrm{H}), 2.22-2.17(\mathrm{~m}, 1 \mathrm{H}) ;{ }^{13} \mathrm{C}$ NMR $\left(101 \mathrm{MHz}, \mathrm{CDCl}_{3}\right) \delta$ 174.3, 171.9, 161.0, 141.0, 137.5, 129.1, 128.1, 128.0, 105.8, 99.0, 93.6, 55.5, 54.7, 46.4, 44.7, 41.0, 40.7, 40.3. IR 3325 (w), 2930 (w), 2839 (w), 2163 (w), 2059 (w), 1781 (s), 1658 (m), 1598 (s), 1538 (m), 1536 (m), 1457 (m), 1429 (m), 1361 (w), 1332 (w), 1330 (w), 1297 (w), 1296 (w), 1265 (m), 1205 (s), 1182 (m), 1157 (s), 1112 (w), 1111 (w), 1099 (w), 1062 (m), 1014 (s), 947 (w), 875 (w), 873 (w), $858(\mathrm{w}), 833(\mathrm{~m}), 814(\mathrm{~m}), 812(\mathrm{~m}), 804(\mathrm{~m}), 769(\mathrm{w}), 767$ (w), $750(\mathrm{~m}), 731(\mathrm{~m}), 688(\mathrm{~m})$, $658(\mathrm{~s})$.

HRMS (ESI/QTOF): Calcd for $\mathrm{C}_{23} \mathrm{H}_{24} \mathrm{INNaO}_{5}{ }^{+}[\mathrm{M}+\mathrm{Na}]^{+}$544.0591; Found 544.0597.
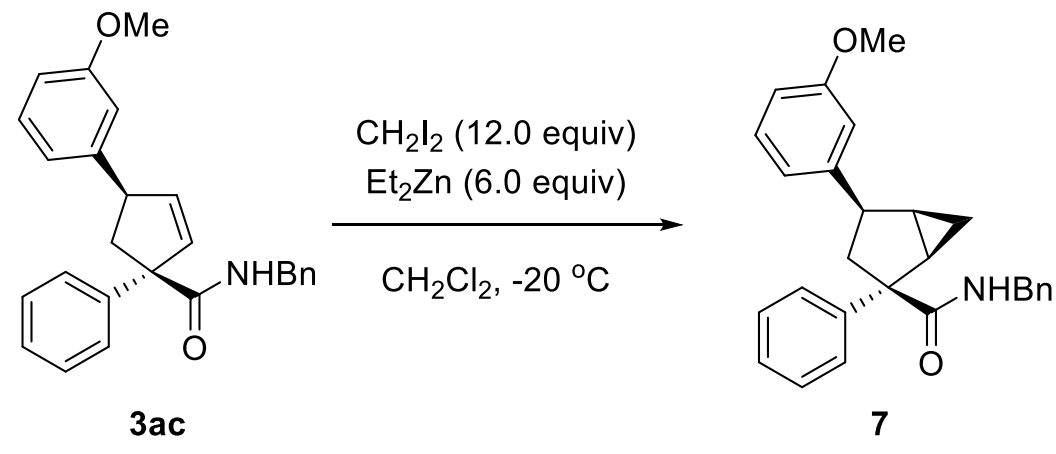

To a solution of $3 \mathrm{ac}(26.8 \mathrm{mg}, 0.07 \mathrm{mmol})$ in $\mathrm{CH}_{2} \mathrm{Cl}_{2}(2 \mathrm{~mL})$ at $-20{ }^{\circ} \mathrm{C}$ was added a solution of $\mathrm{Et}_{2} \mathrm{Zn}$ in hexane $(1.0 \mathrm{M}, 0.42 \mathrm{~mL}, 0.42 \mathrm{mmol})$. After stirring for $10 \mathrm{~min}, \mathrm{CH}_{2} \mathrm{I}_{2}(0.068 \mathrm{~mL}, 0.84$ mmol) was added. Then the mixture was allowed to stir at this temperature for $48 \mathrm{hrs}$. Saturated $\mathrm{NH}_{4} \mathrm{Cl}$ solution was added to quench the reaction and the reaction mixture was extracted with 
$\mathrm{CH}_{2} \mathrm{Cl}_{2}$. The organic layers were dried over anhydrous $\mathrm{Na}_{2} \mathrm{SO}_{4}$ and concentrated. The residue was purified by flash chromatography (silica gel, eluted with petroleum ether/ethyl acetate) to give product 7 (15.6 mg, 56\% yield) as colorless oil. $[\alpha]_{D}^{25}-25.7\left(c 0.78, \mathrm{CHCl}_{3}\right)$.

${ }^{1} \mathbf{H}$ NMR $\left(400 \mathrm{MHz}, \mathrm{CDCl}_{3}\right) \delta$ 7.56-7.54 (m, 2H), $7.39(\mathrm{t}, J=7.3 \mathrm{~Hz}, 2 \mathrm{H}), 7.32-7.23(\mathrm{~m}, 4 \mathrm{H})$, 7.22-7.17 (m, 3H), $6.90(\mathrm{~d}, J=7.7 \mathrm{~Hz}, 1 \mathrm{H}), 6.87(\mathrm{t}, J=5.0 \mathrm{~Hz}, 1 \mathrm{H}), 6.74(\mathrm{dd}, J=8.1,2.1 \mathrm{~Hz}$, $1 \mathrm{H}), 5.74(\mathrm{t}, J=4.7 \mathrm{~Hz}, 1 \mathrm{H}), 4.53(\mathrm{dd}, J=15.0,6.0 \mathrm{~Hz}, 1 \mathrm{H}), 4.38(\mathrm{dd}, J=15.0,5.5 \mathrm{~Hz}, 1 \mathrm{H})$, $3.78(\mathrm{~s}, 3 \mathrm{H}), 3.32(\mathrm{ddd}, J=11.7,7.4,4.1 \mathrm{~Hz}, 1 \mathrm{H}), 2.36(\mathrm{dd}, J=13.5,12.3 \mathrm{~Hz}, 1 \mathrm{H}), 2.13-2.05$ $(\mathrm{m}, 1 \mathrm{H}), 1.90-1.81(\mathrm{~m}, 2 \mathrm{H}), 0.97-0.94(\mathrm{~m}, 1 \mathrm{H}), 0.76(\mathrm{dt}, J=7.9,5.8 \mathrm{~Hz}, 1 \mathrm{H}) ;{ }^{13} \mathrm{C}$ NMR $(101$ $\left.\mathrm{MHz}_{\mathrm{CDCl}}\right) \delta 175.1,159.7,144.7,144.2,138.7,129.3,128.9,128.7,127.6,127.5,127.3$, 126.9, 119.9, 113.3, 111.6, 58.9, 55.3, 43.8, 42.2, 39.1, 23.6, 22.5, 5.3. IR $3791(\mathrm{w}), 3649(\mathrm{w})$, 3364 (w), 3362 (w), 3344 (w), 3319 (w), 3061 (w), 3022 (w), 3001 (w), 2961 (w), 2959 (w), 2924 (w), 2922 (w), 2525 (w), 2307 (w), 2161 (s), 1801 (w), 1650 (s), 1604 (m), 1585 (m), 1517 (s), 1494 (s), 1469 (w), 1454 (m), 1361 (w), 1359 (w), 1342 (w), 1340 (w), 1317 (w), 1315 (w), 1263 (m), 1191 (w), 1159 (w), 1120 (w), 1051 (m), 1049 (m), 989 (w), 987 (w), 866 (w), $833(\mathrm{w}), 831(\mathrm{w}), 791(\mathrm{w}), 777(\mathrm{w}), 756(\mathrm{~m}), 735(\mathrm{~m}), 719(\mathrm{~s}), 698(\mathrm{~s}), 679(\mathrm{w}), 658(\mathrm{w})$. HRMS (ESI/QTOF): Calcd for $\mathrm{C}_{27} \mathrm{H}_{27} \mathrm{NNaO}_{2}{ }^{+}[\mathrm{M}+\mathrm{Na}]^{+} 420.1934$; Found 420.1934.

\section{References}

1. a) Wu, Y.-J.; He, H.; Bertekap, R.; Westphal, R.; Lelas, S.; Newton, A.; Wallace, T.; Taber, M.; Davis, C.; Macor, J. E.; Bronson, J. Bioorg. Med. Chem. 2013, 21, 2217.

b) Hoang, G. L.; Yang, Z.-D.; Smith, S. M.; Pal, R.; Miska, J. L.; Pérez, D. E.; Pelter, L. S. W.; Zeng, X. C.; Takacs, J. M. Org. Lett. 2015, 17, 940.

2. a) Liu, B.; Hu, L. Bioorg. Med. Chem. 2003, 11, 3889.

b) Xu, Z.; Wang, Q.; Zhu, J. Angew. Chem. Int. Ed. 2013, 52, 3272.

3. Kattela, S.; Heerdt, G.; Correia, C. R. D. Adv. Synth. Catal. 2017, 359, 260.

4. Shi, S.-L.; Wei, X.-F.; Shimizu, Y.; Kanai, M. J. Am. Chem. Soc. 2012, 134, 17019. 


\section{Copies of NMR spectra}
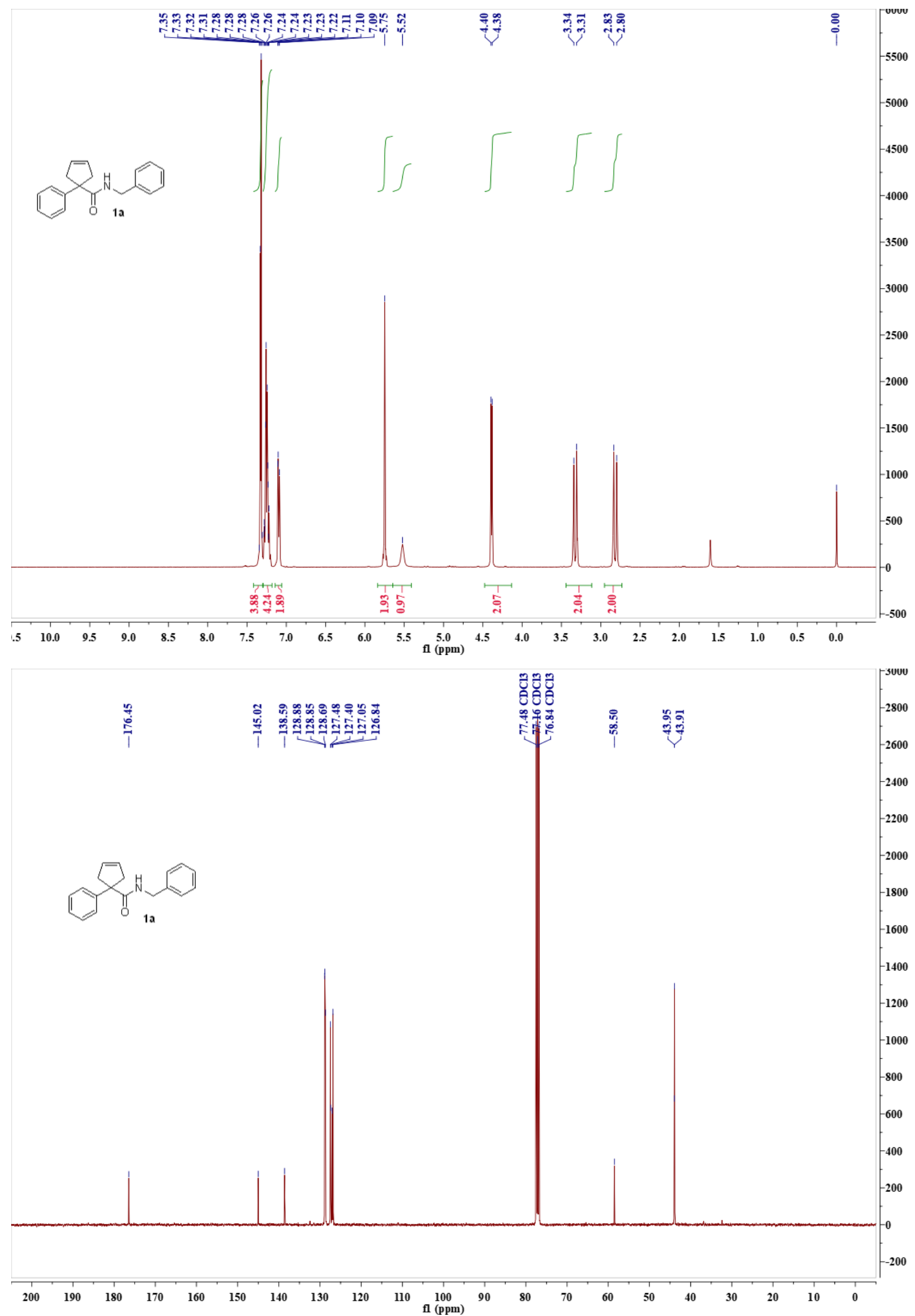

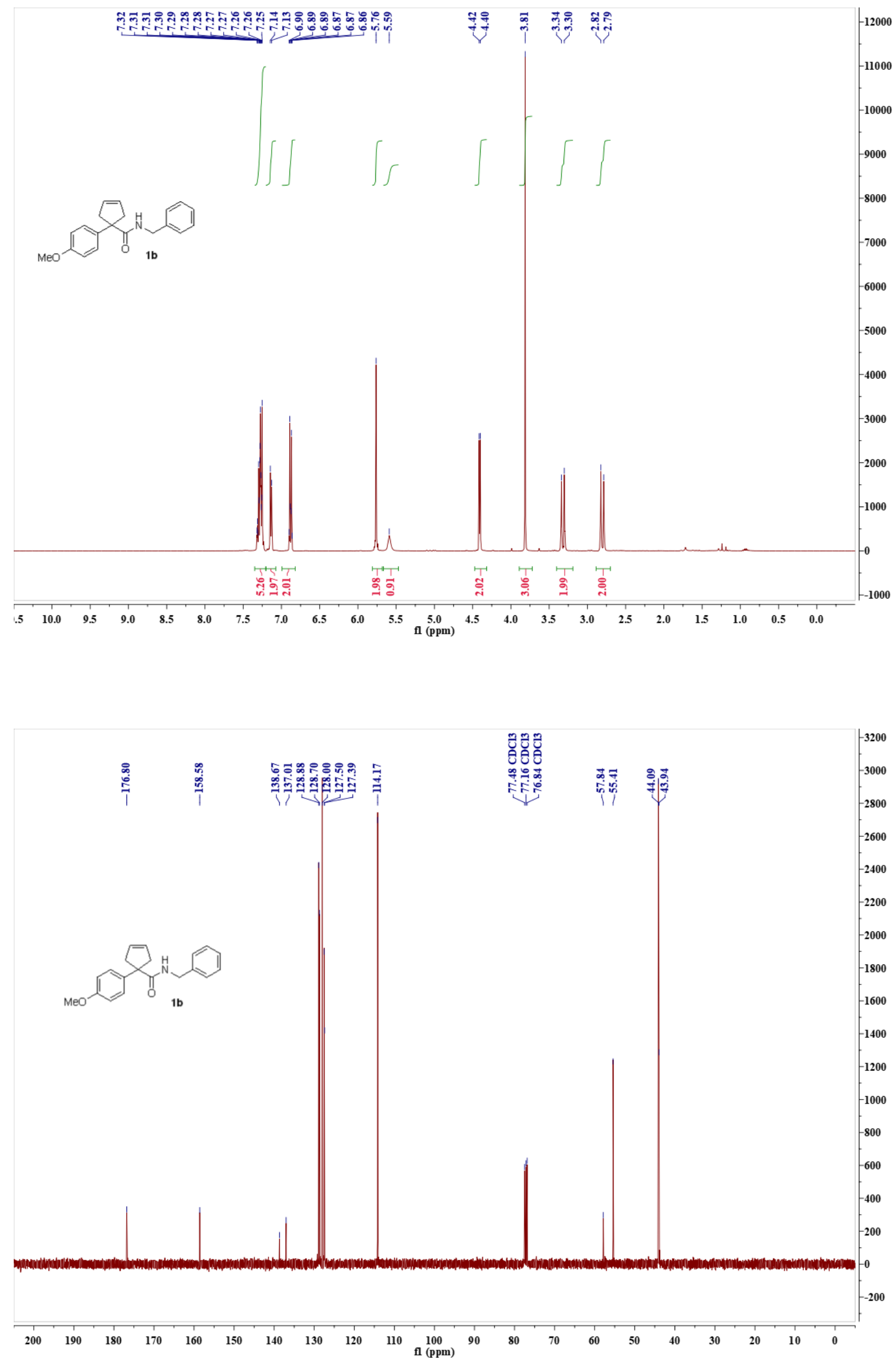

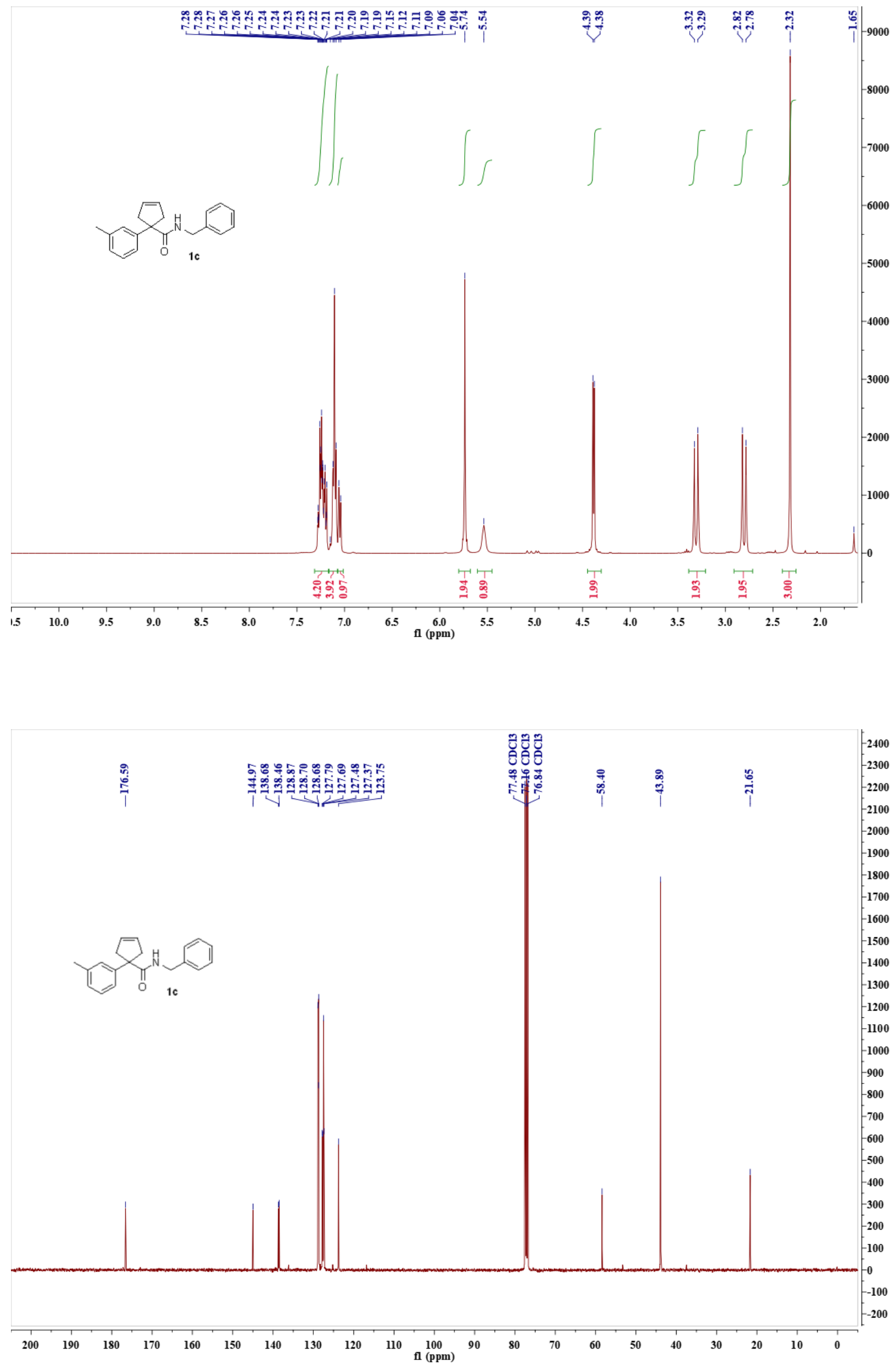

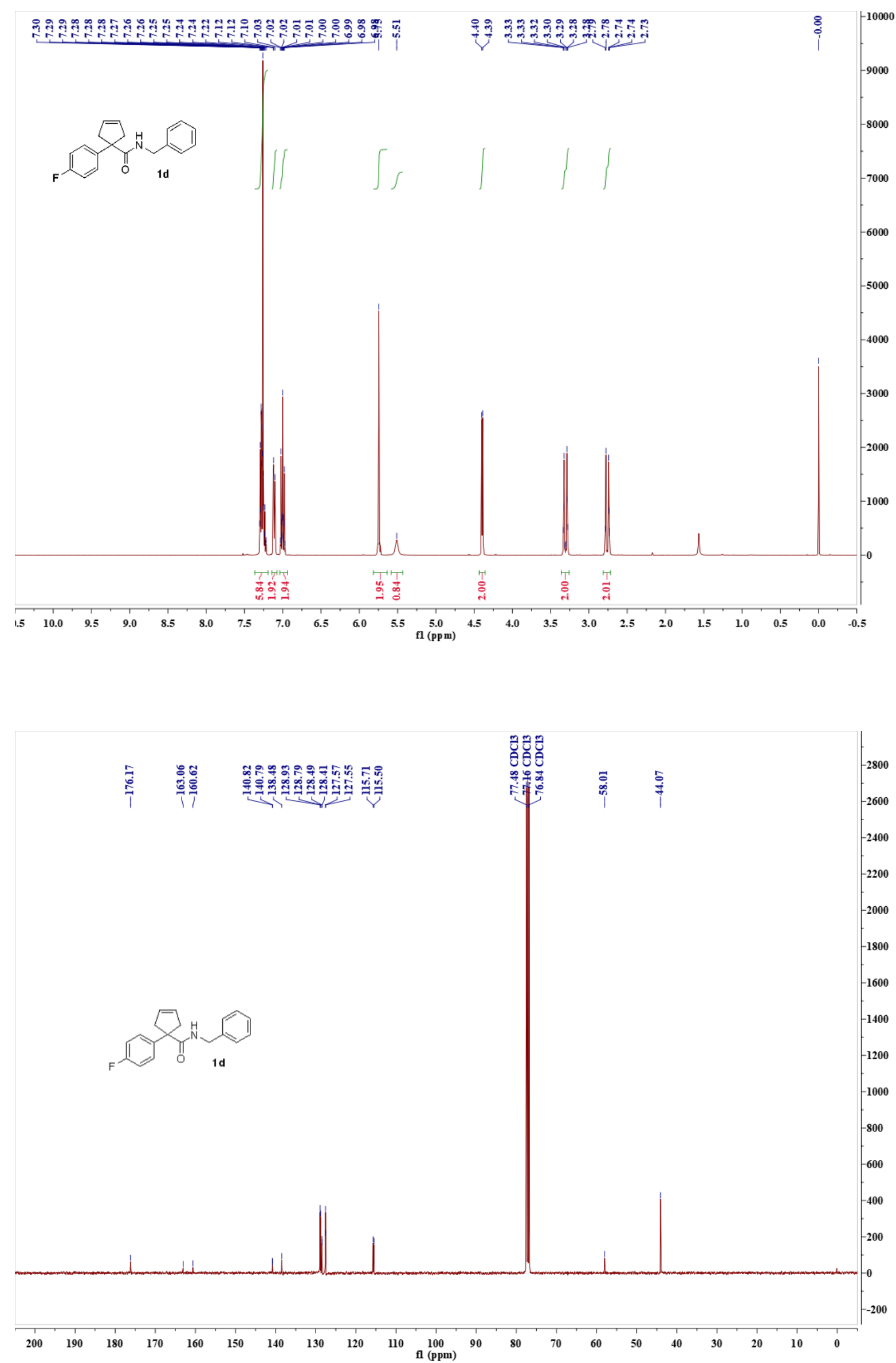

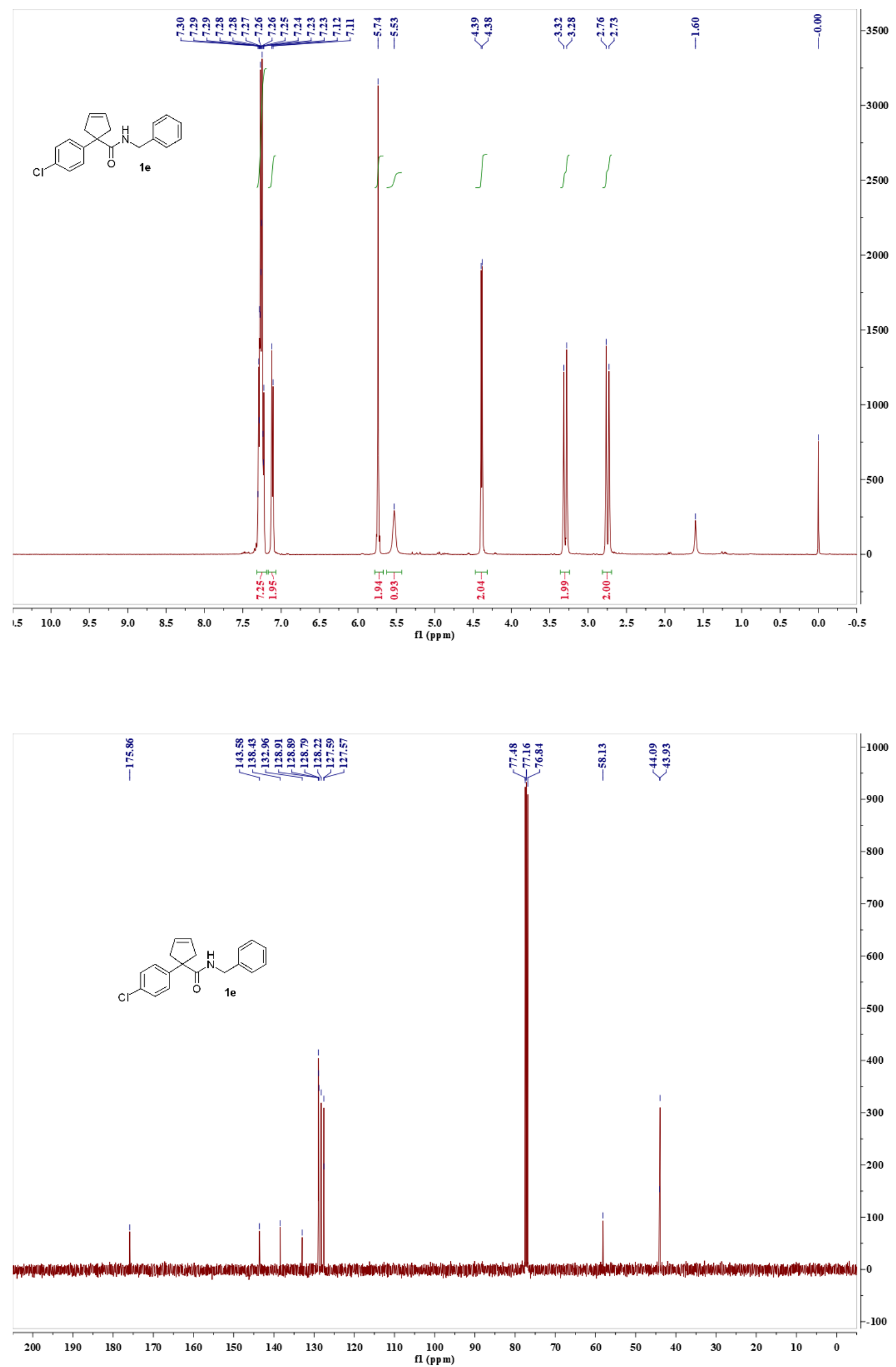

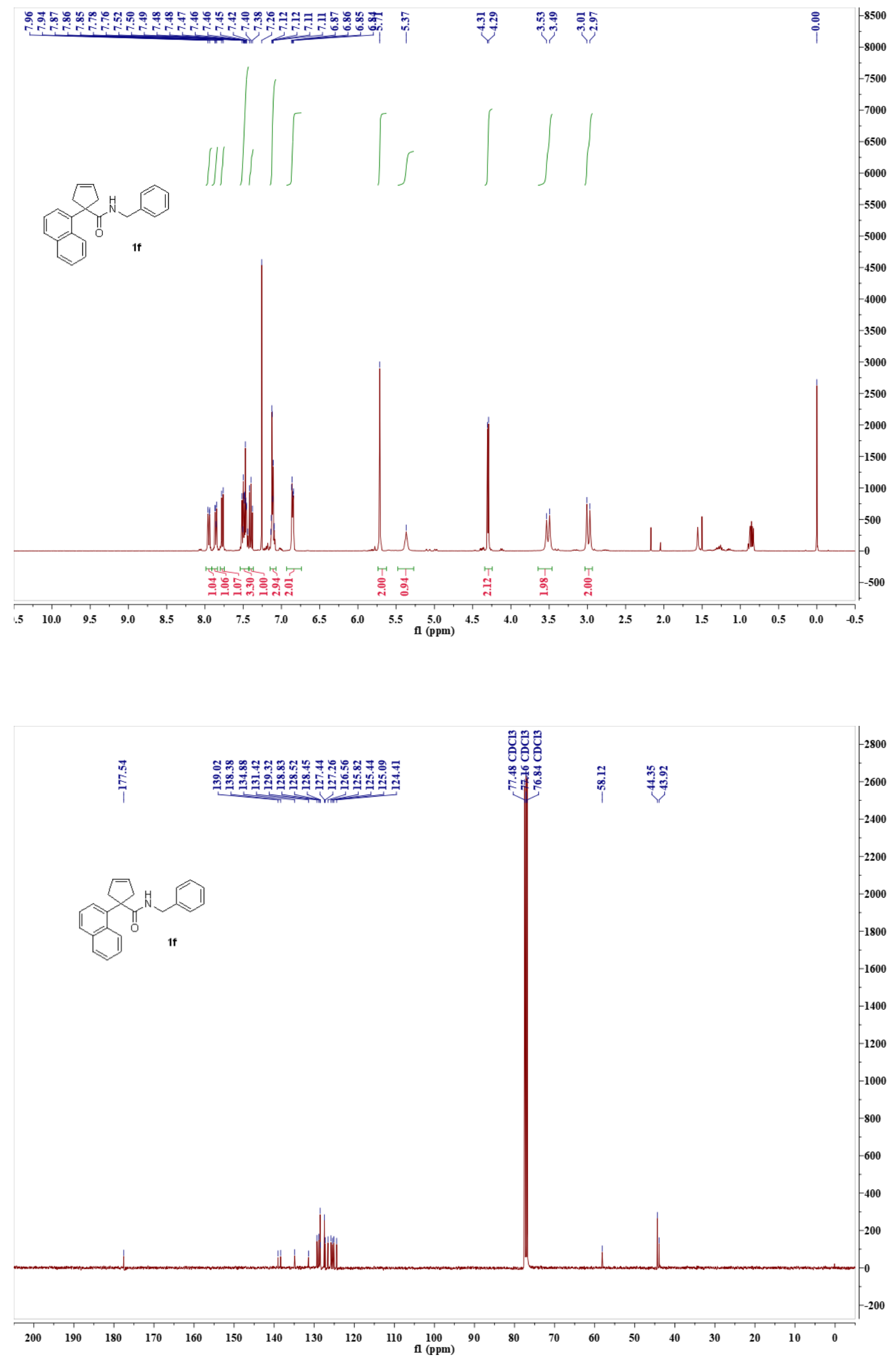

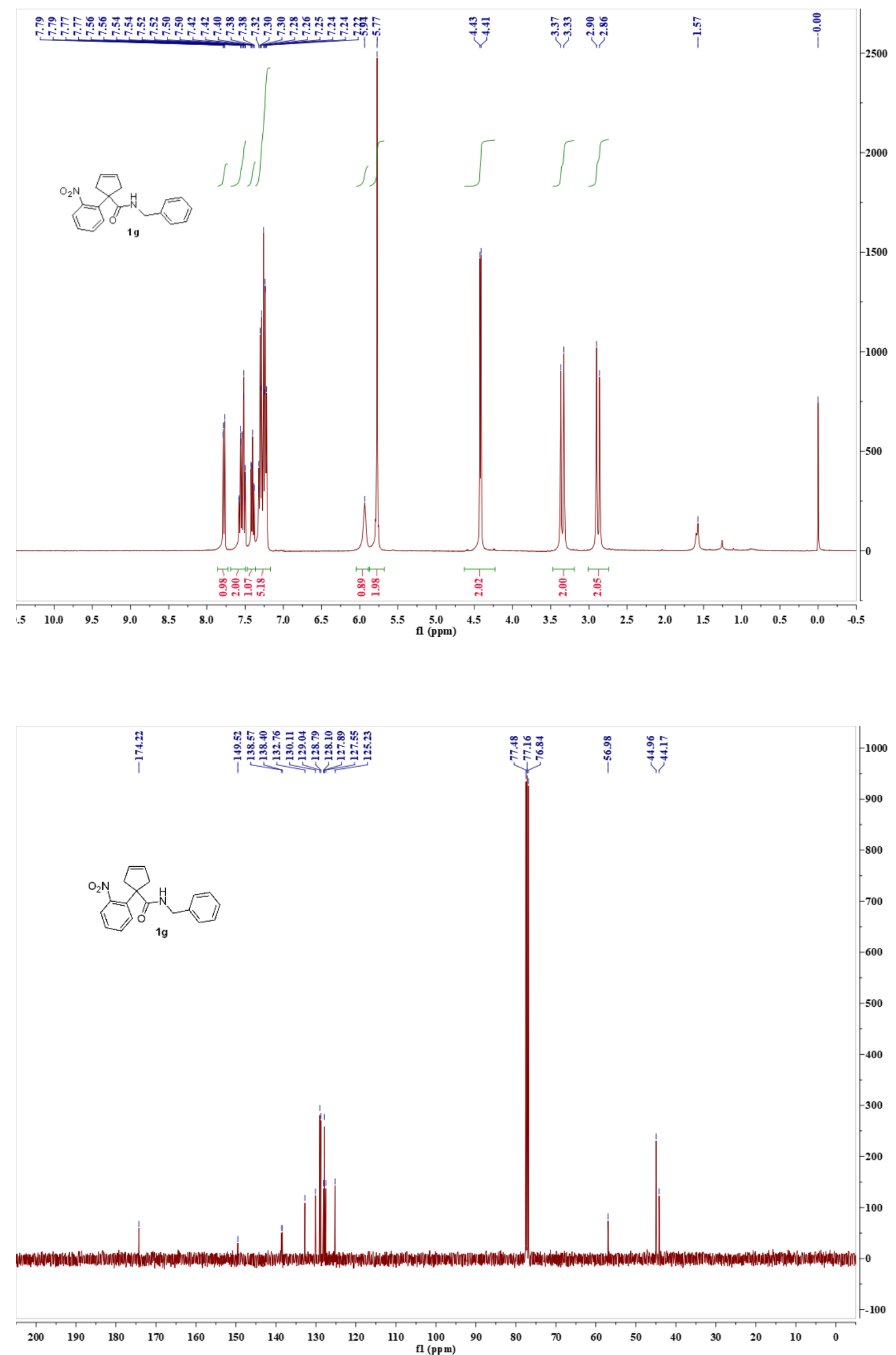

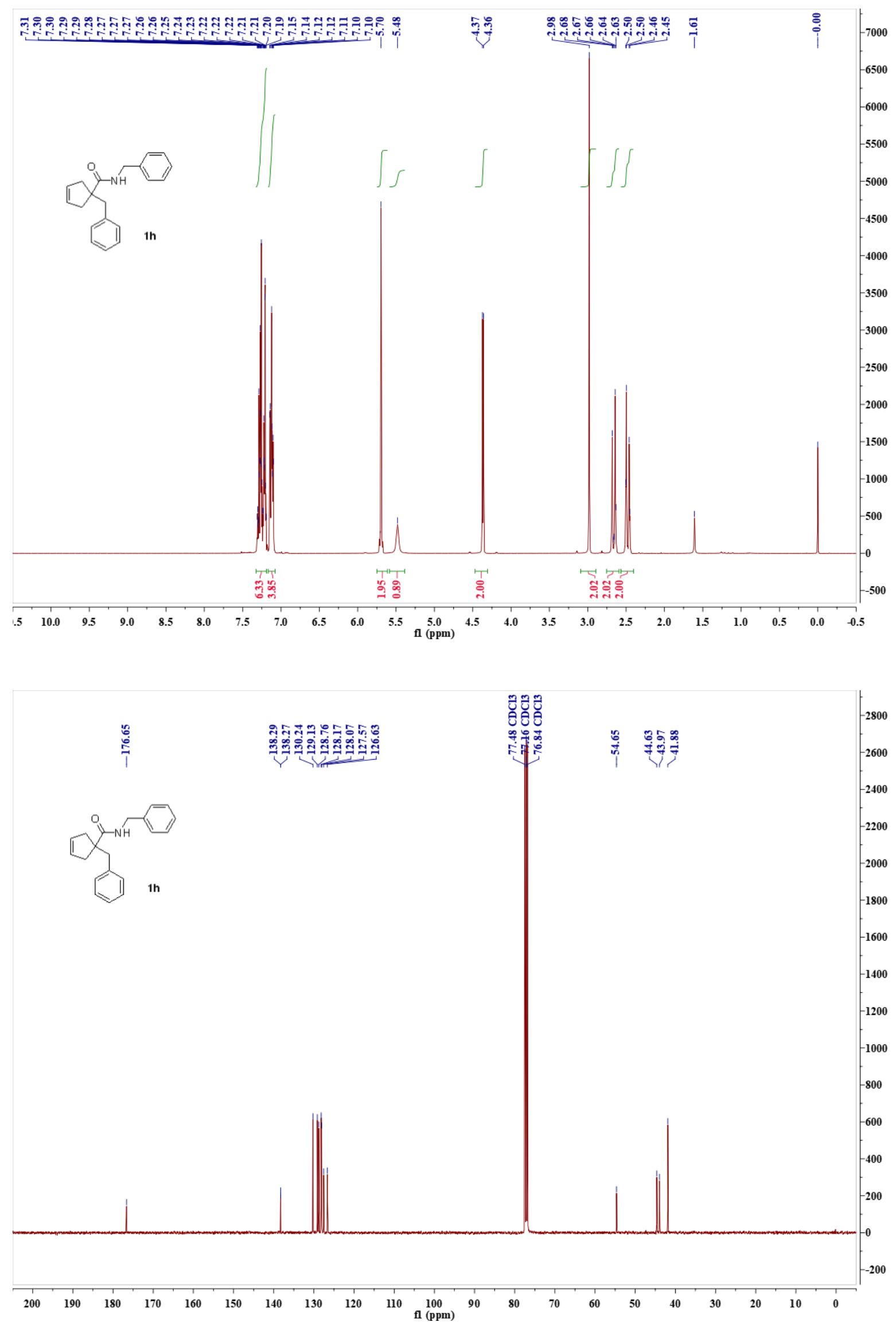

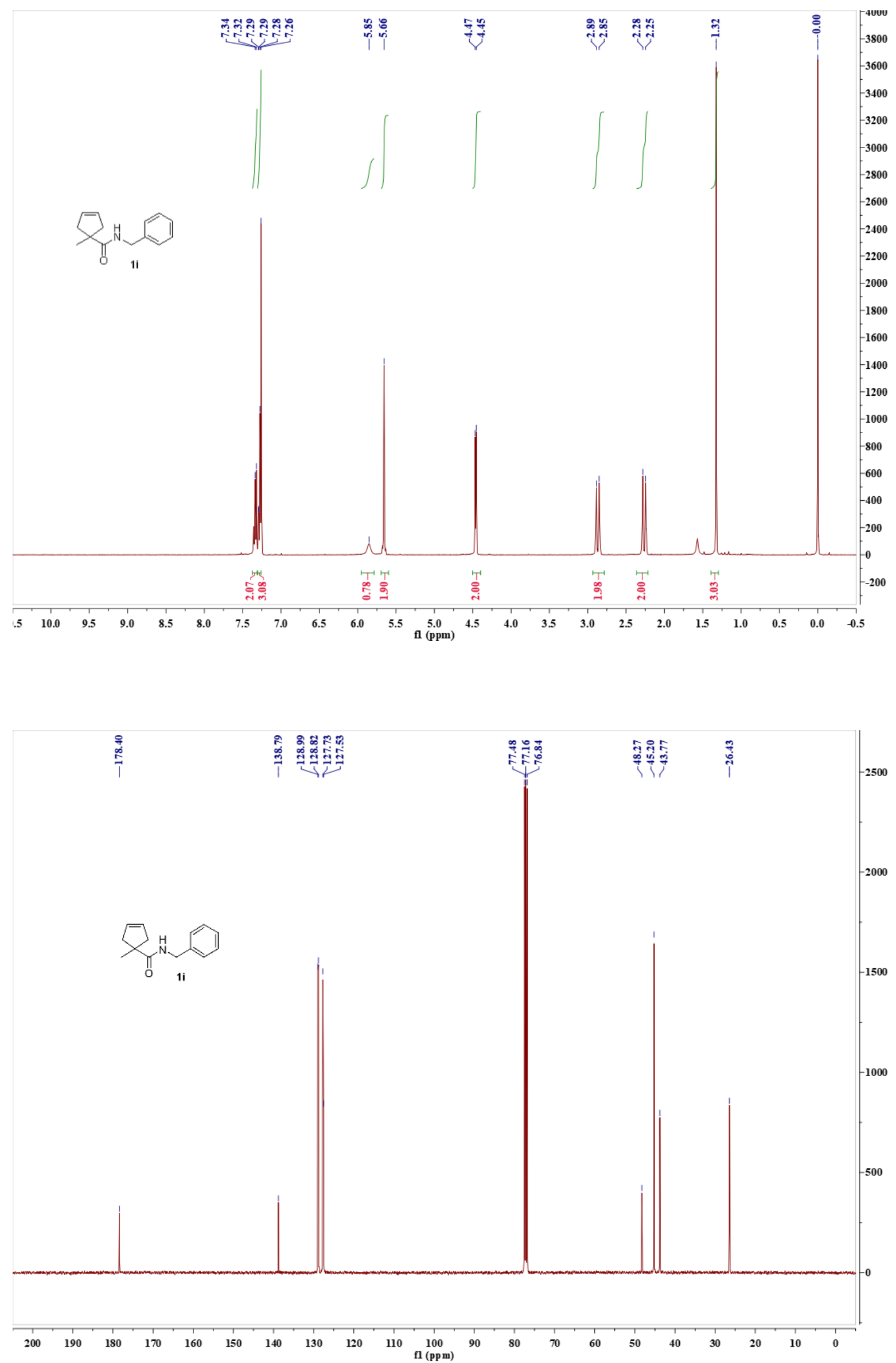

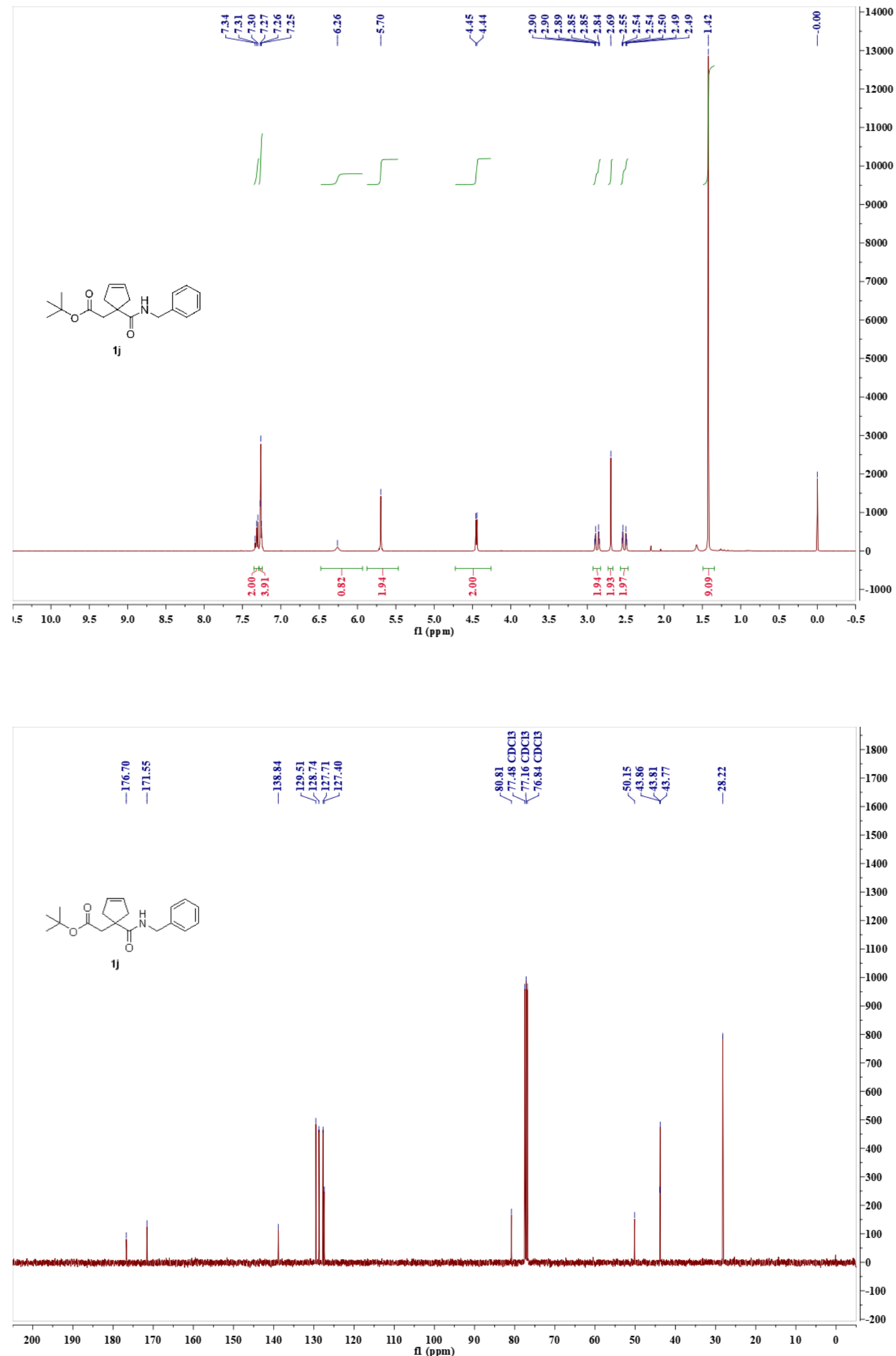

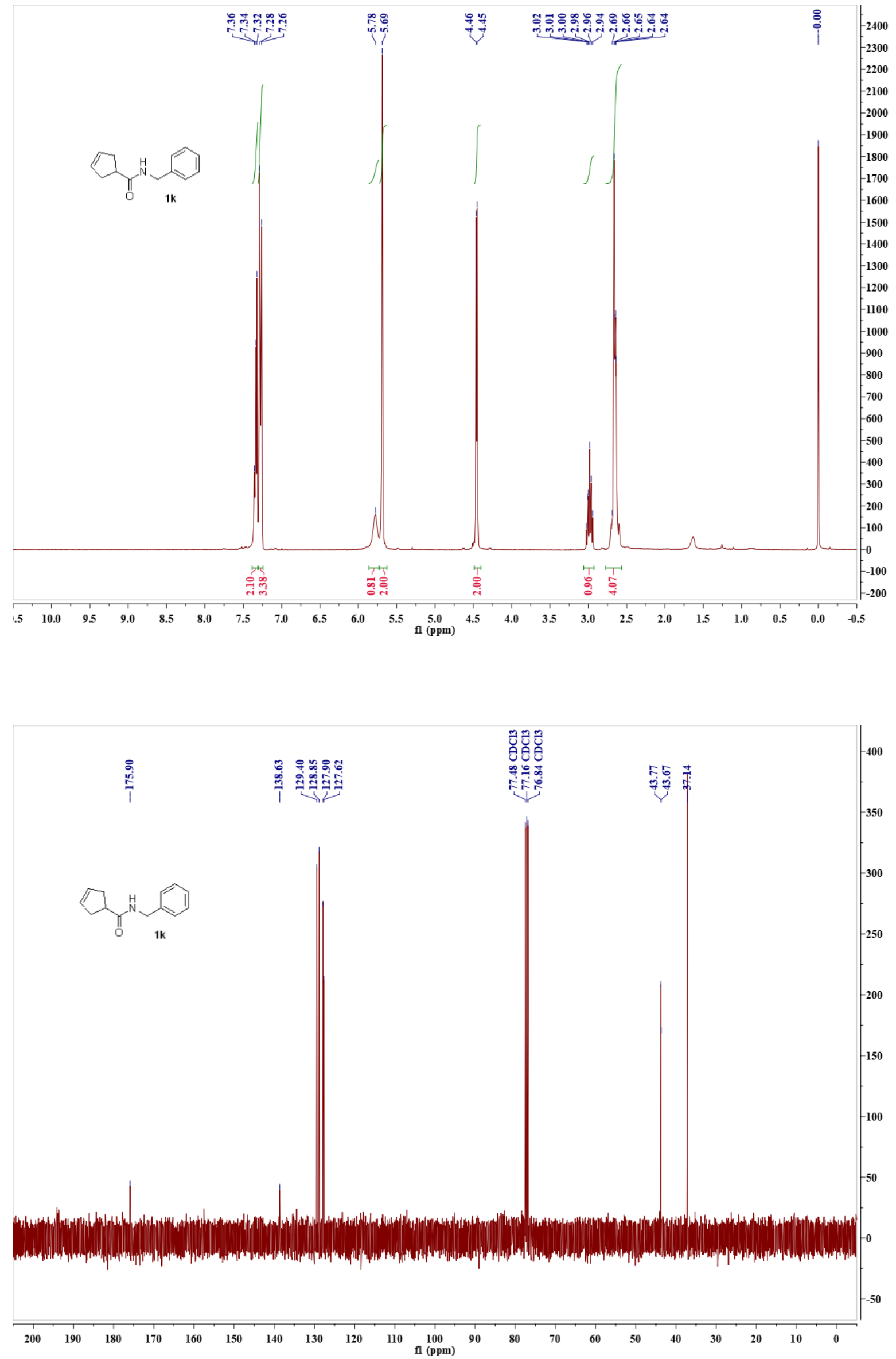

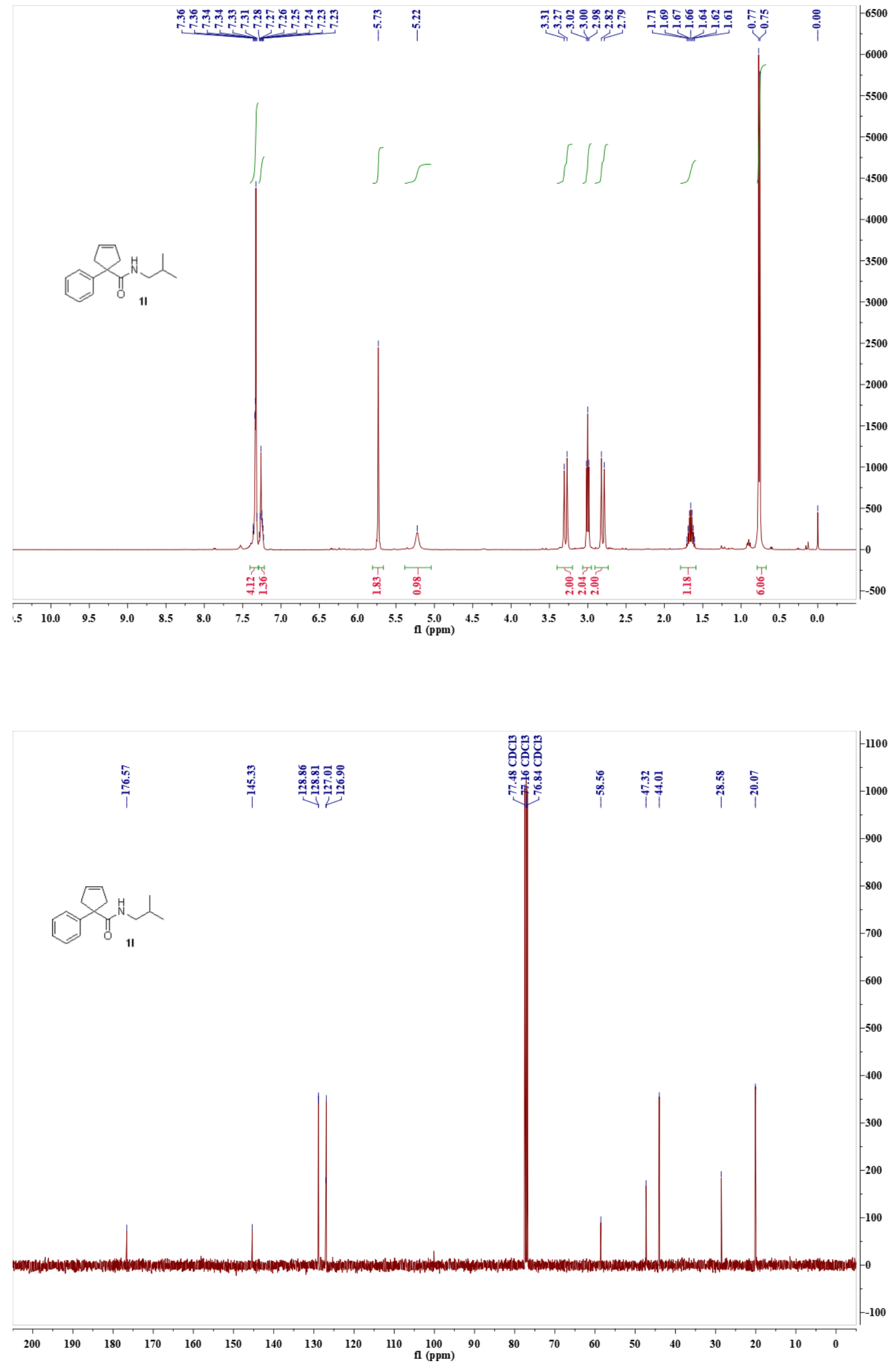

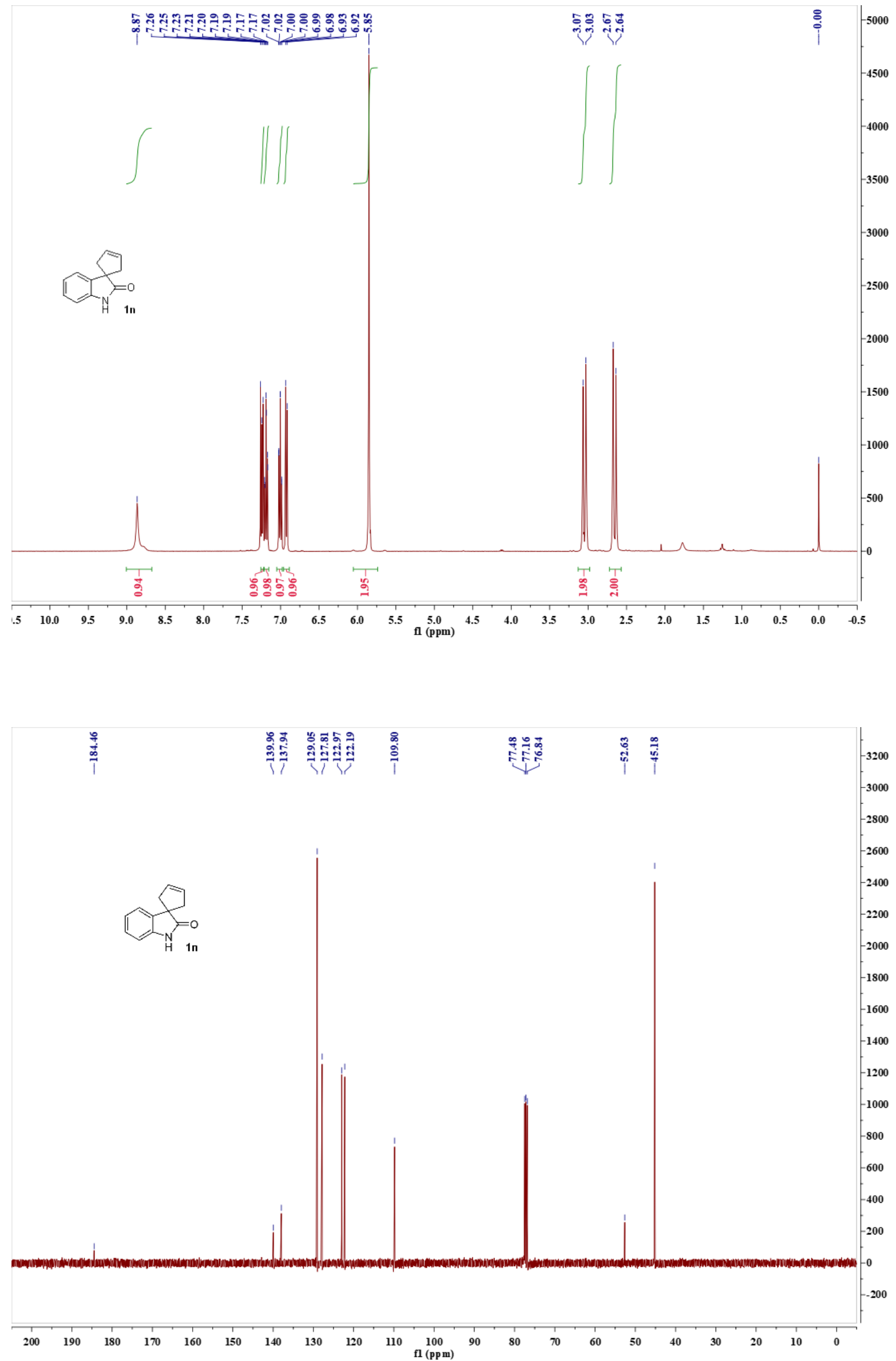

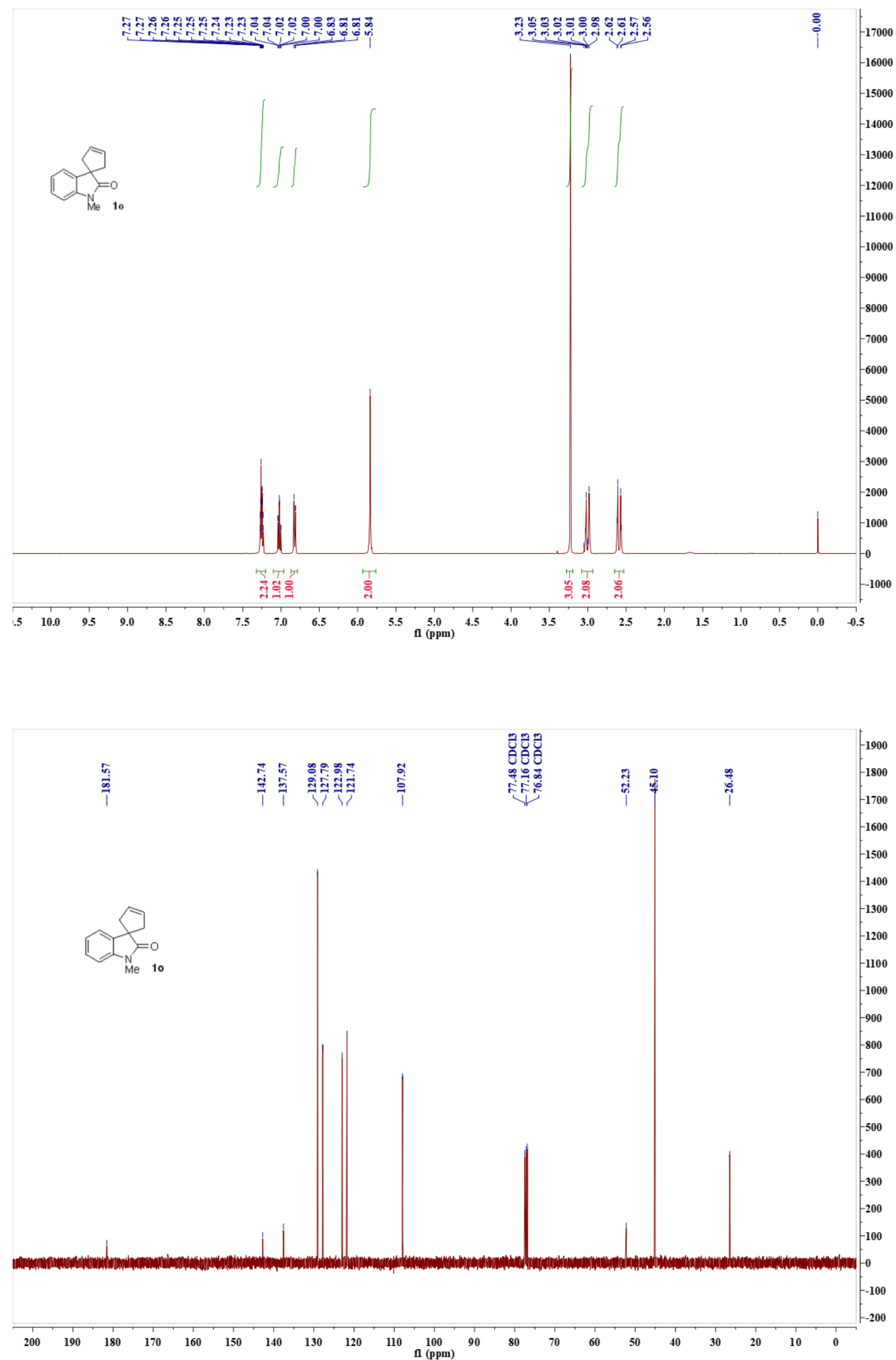

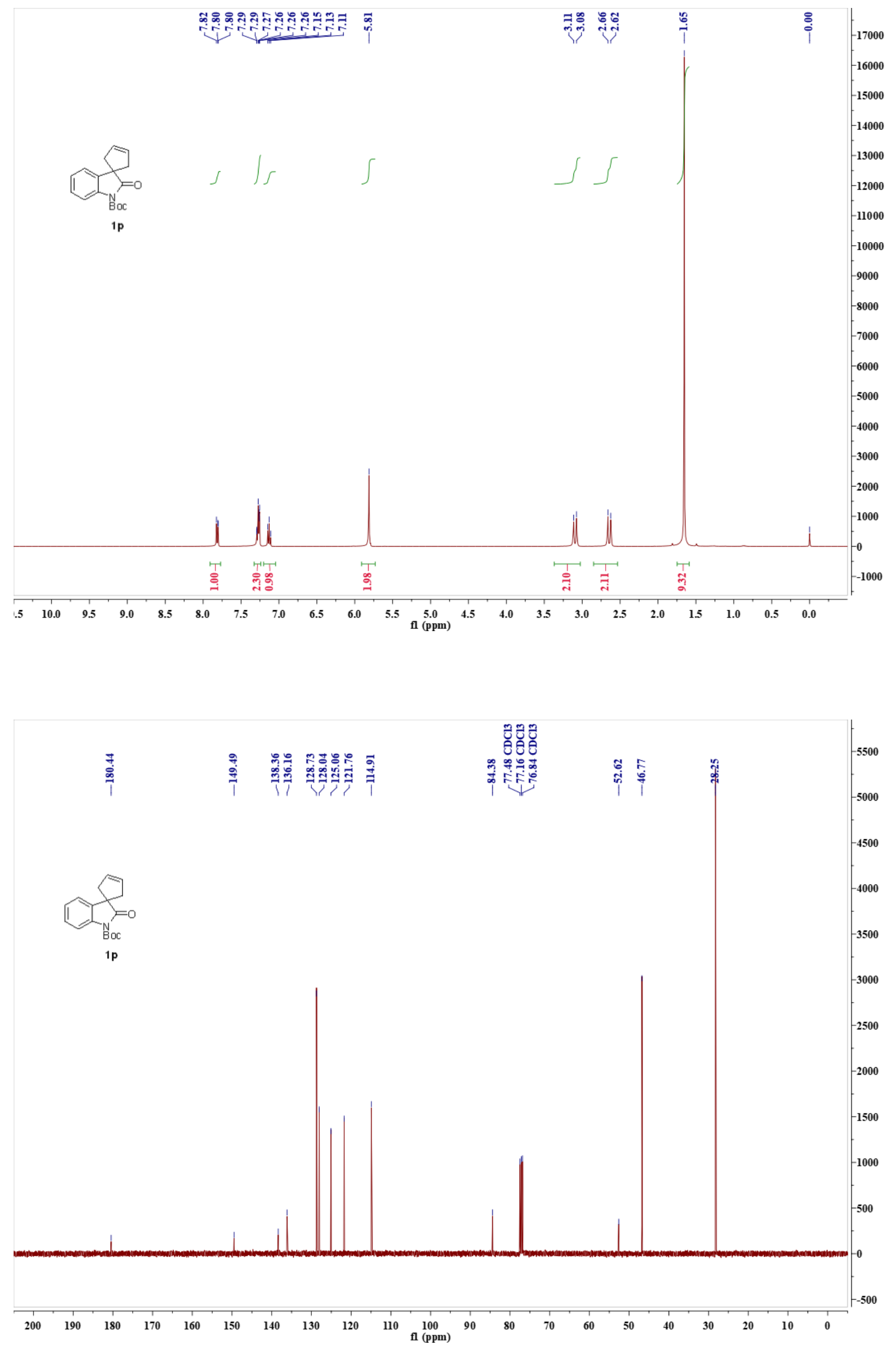

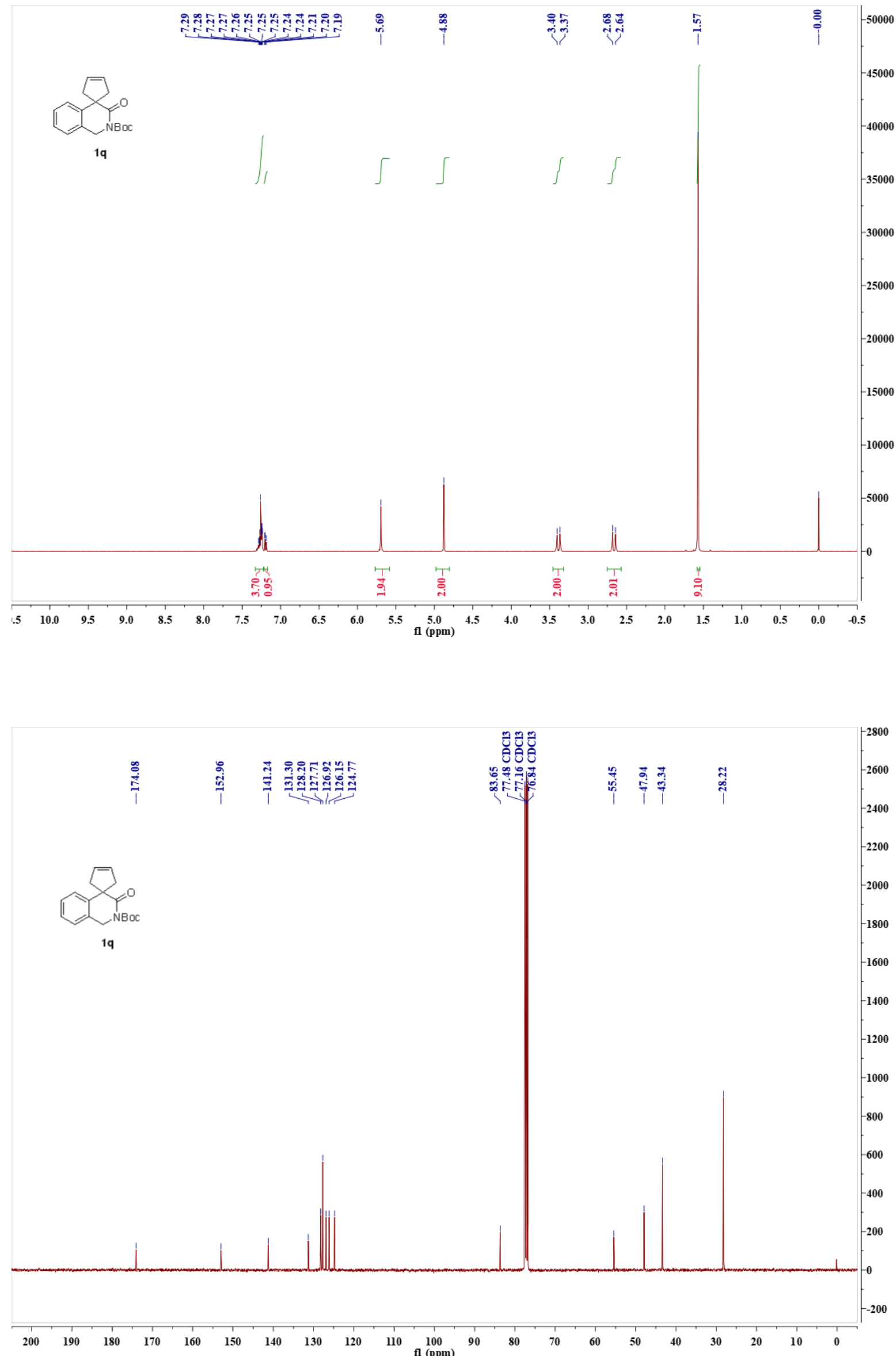

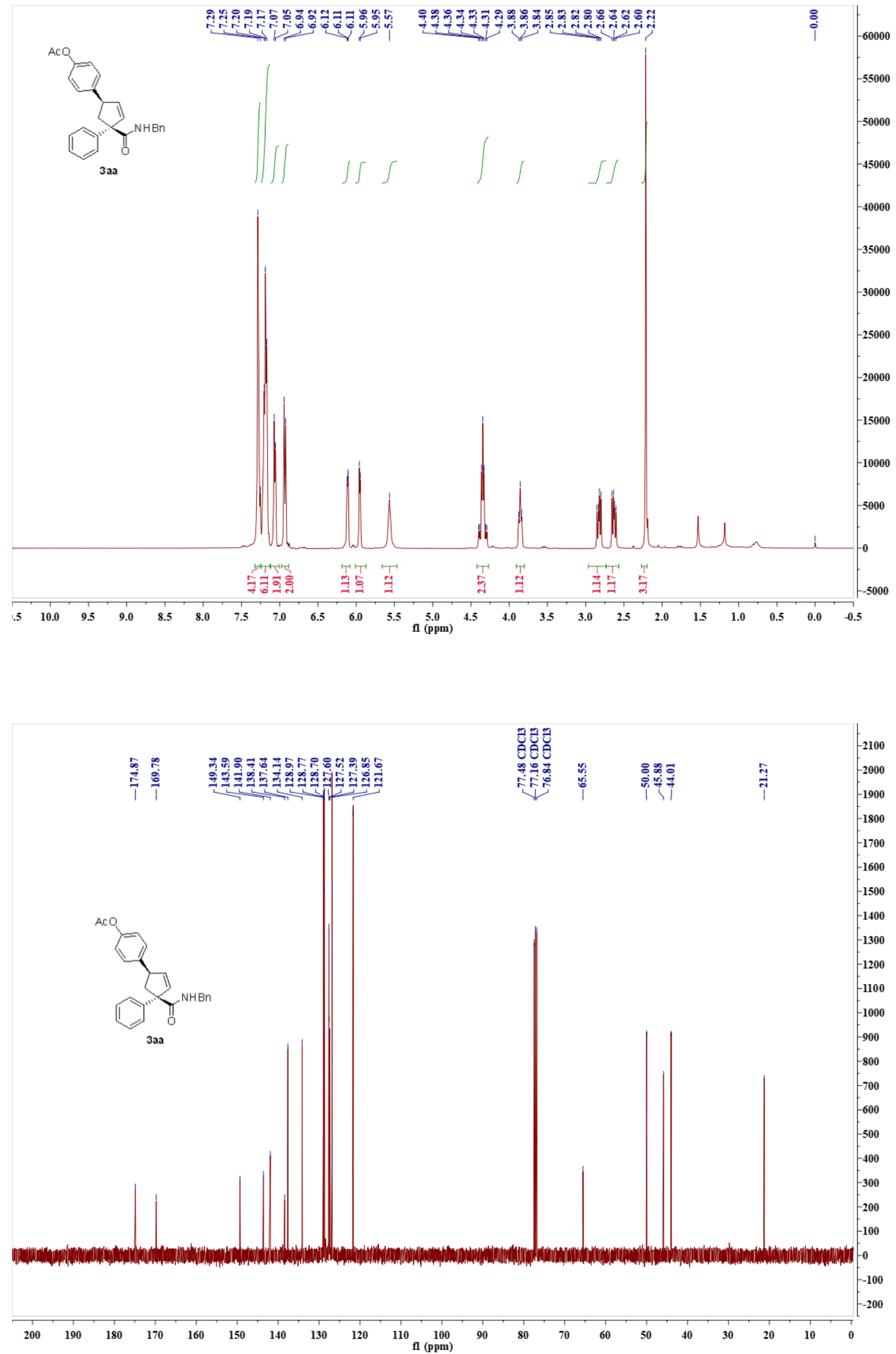

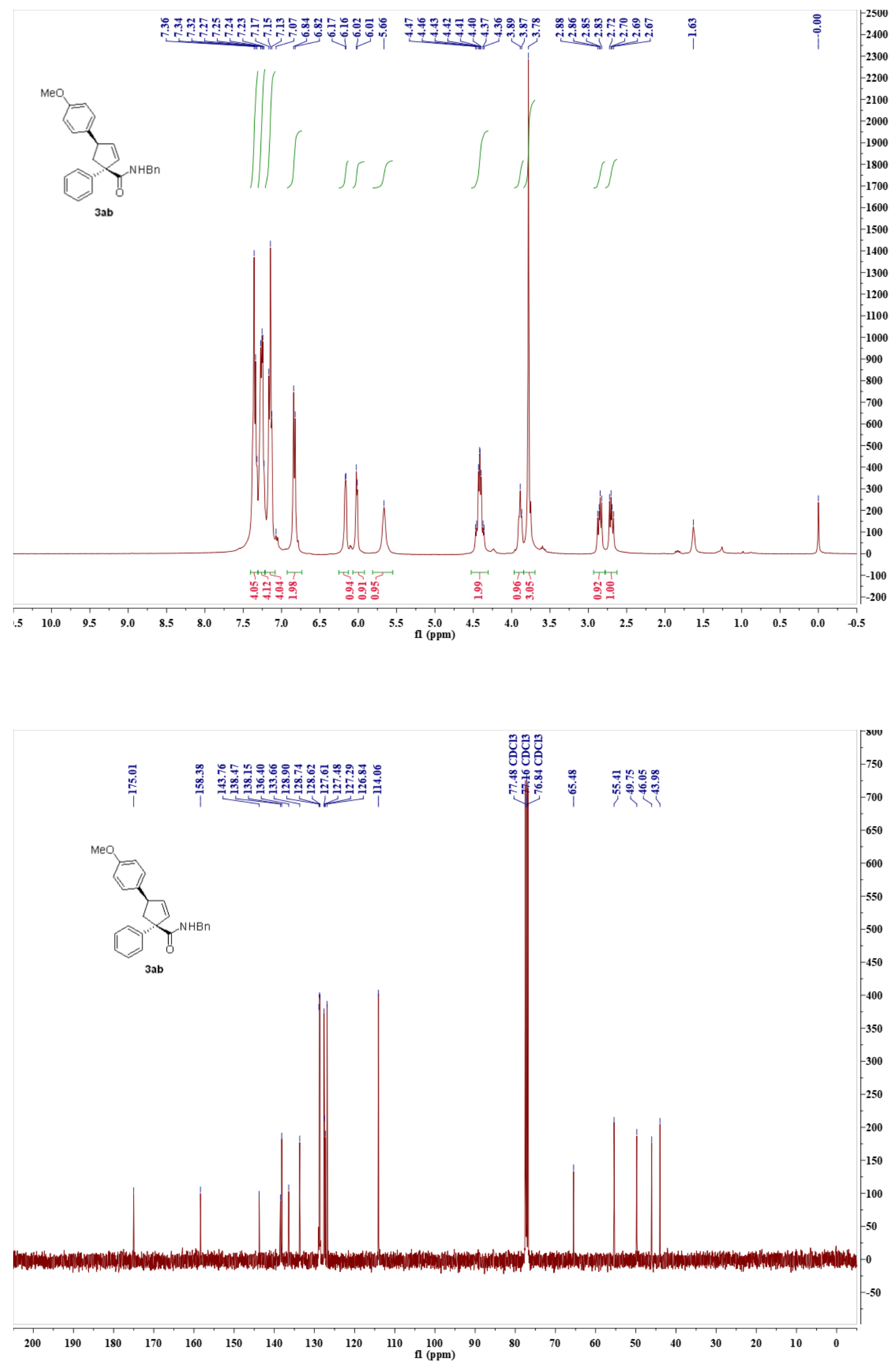

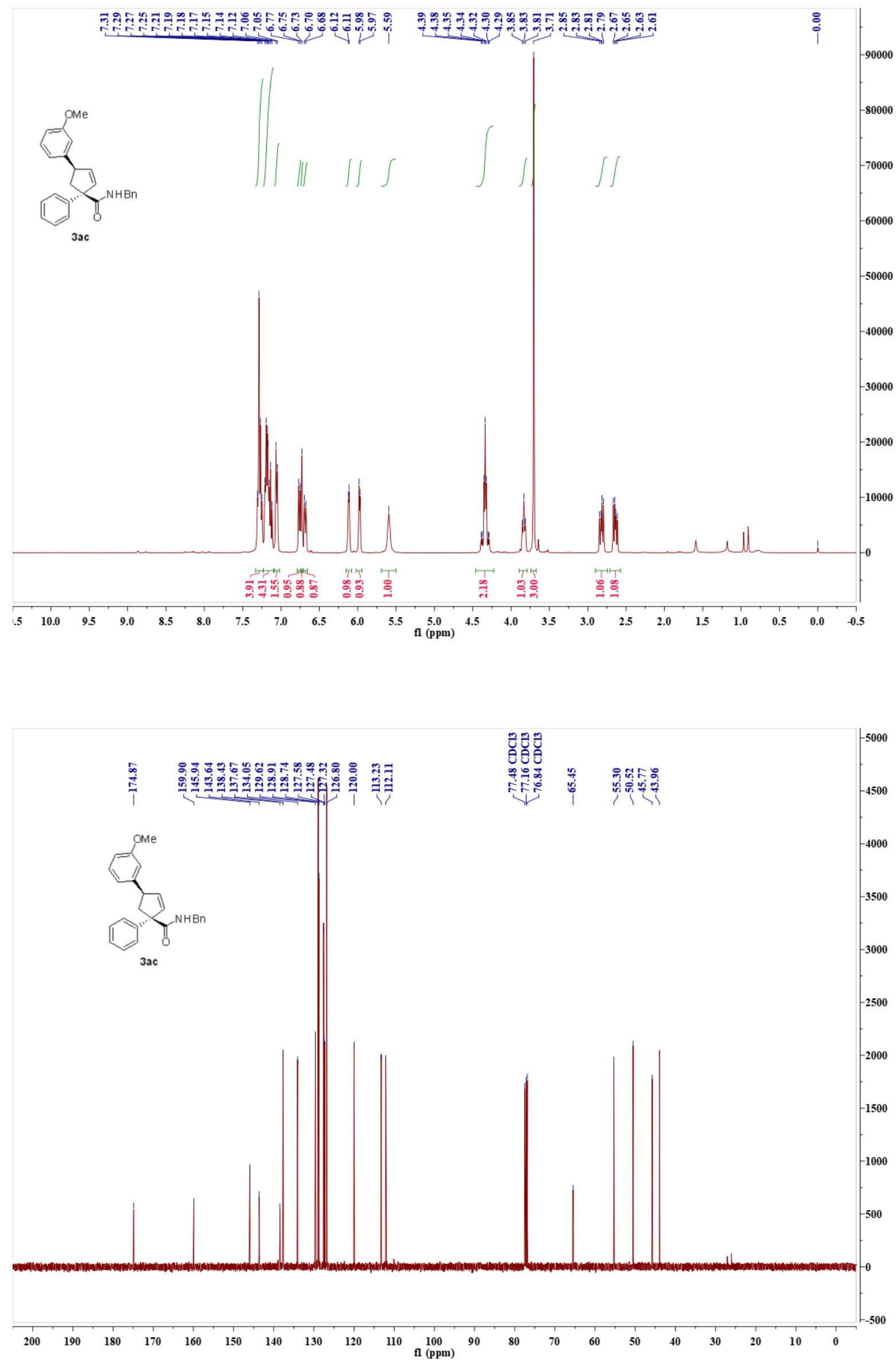

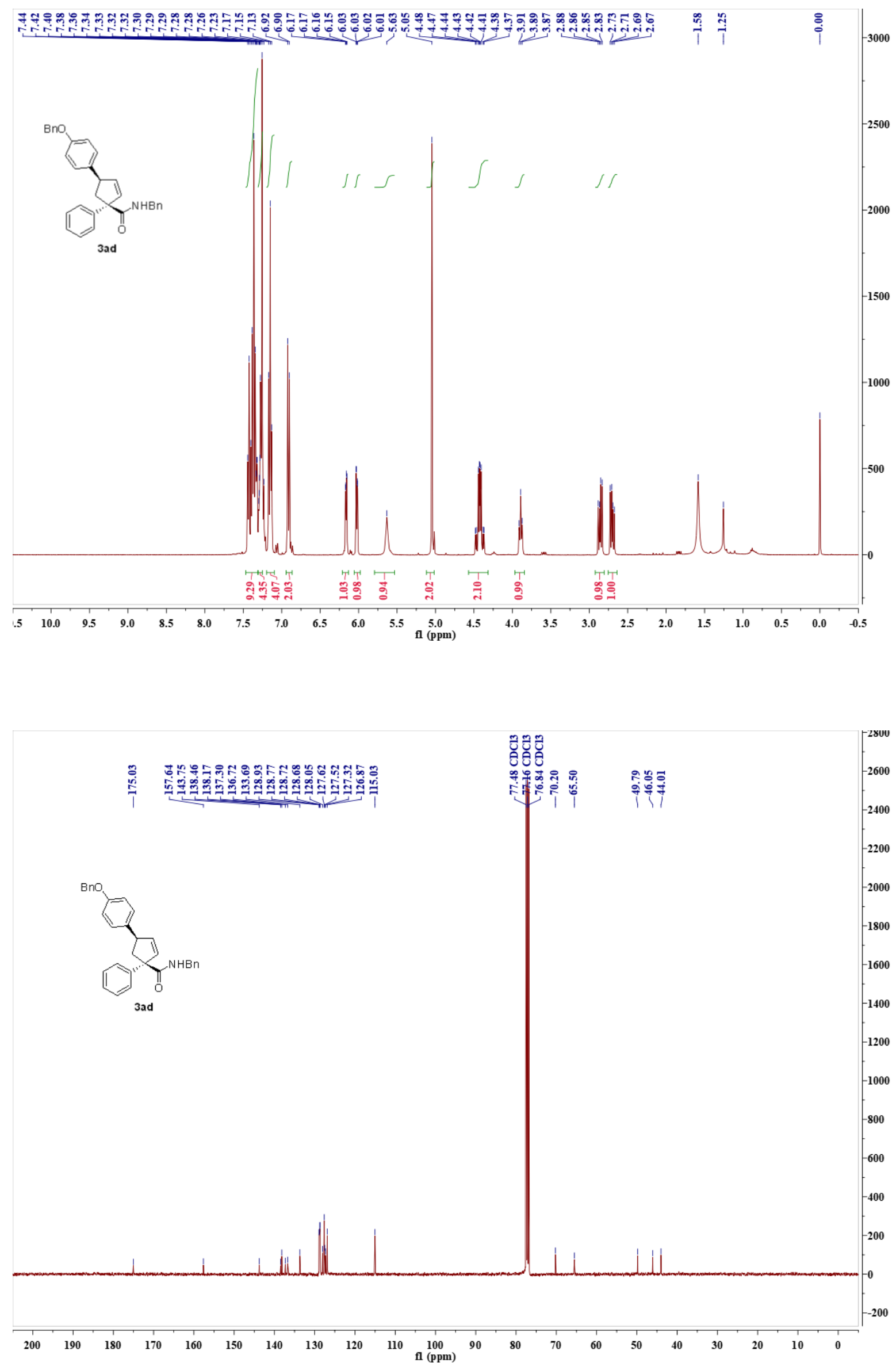

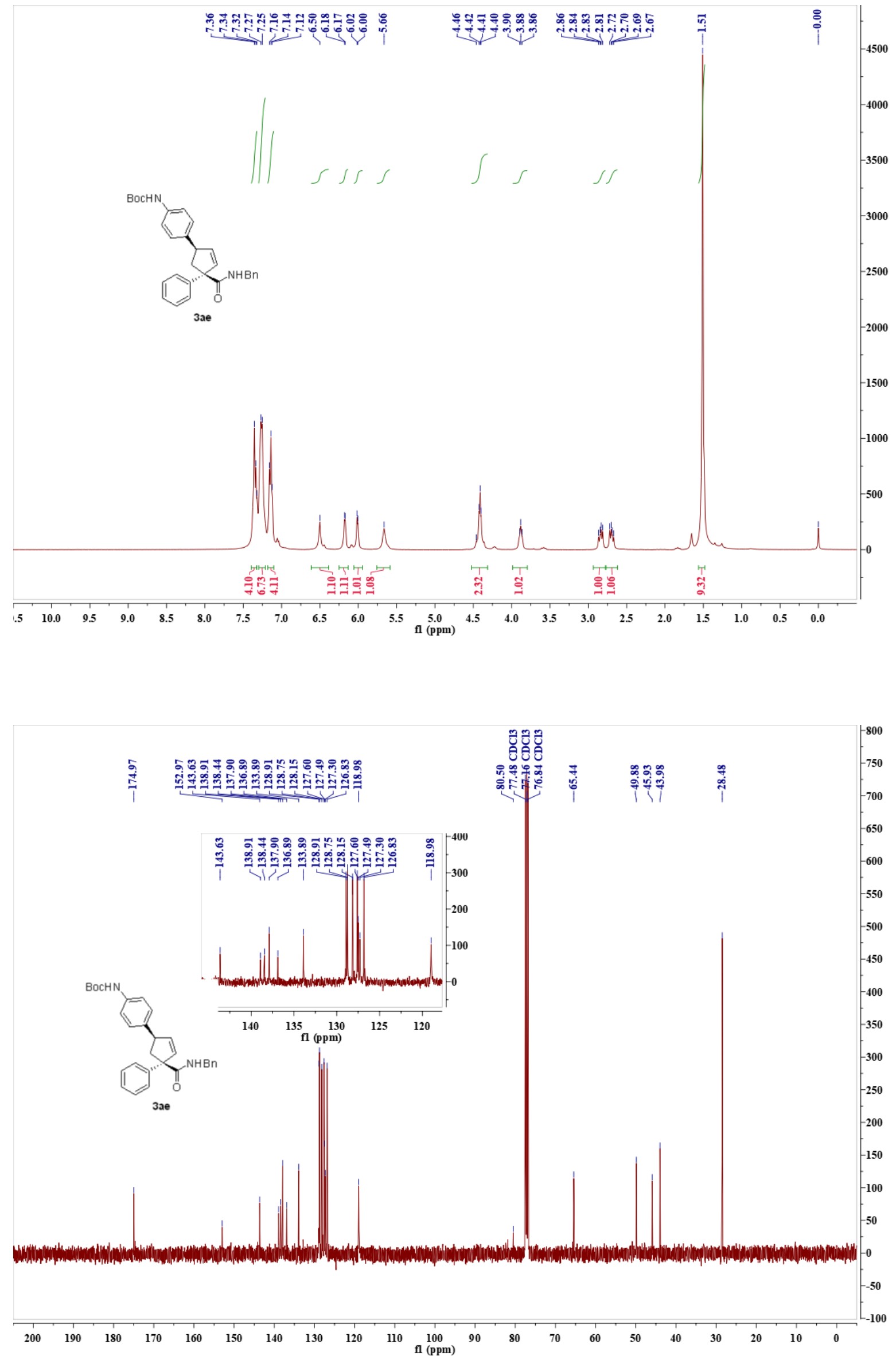

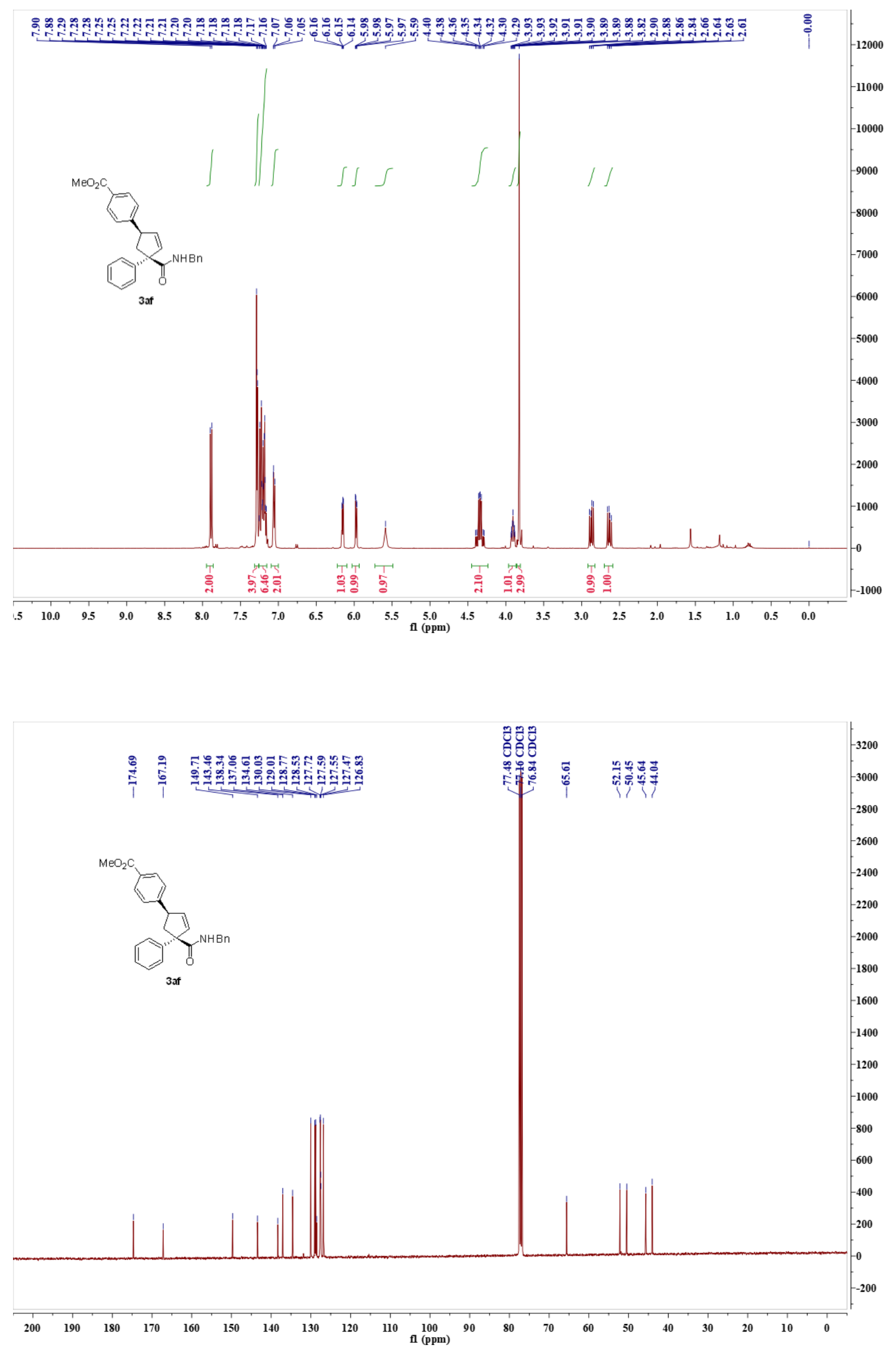

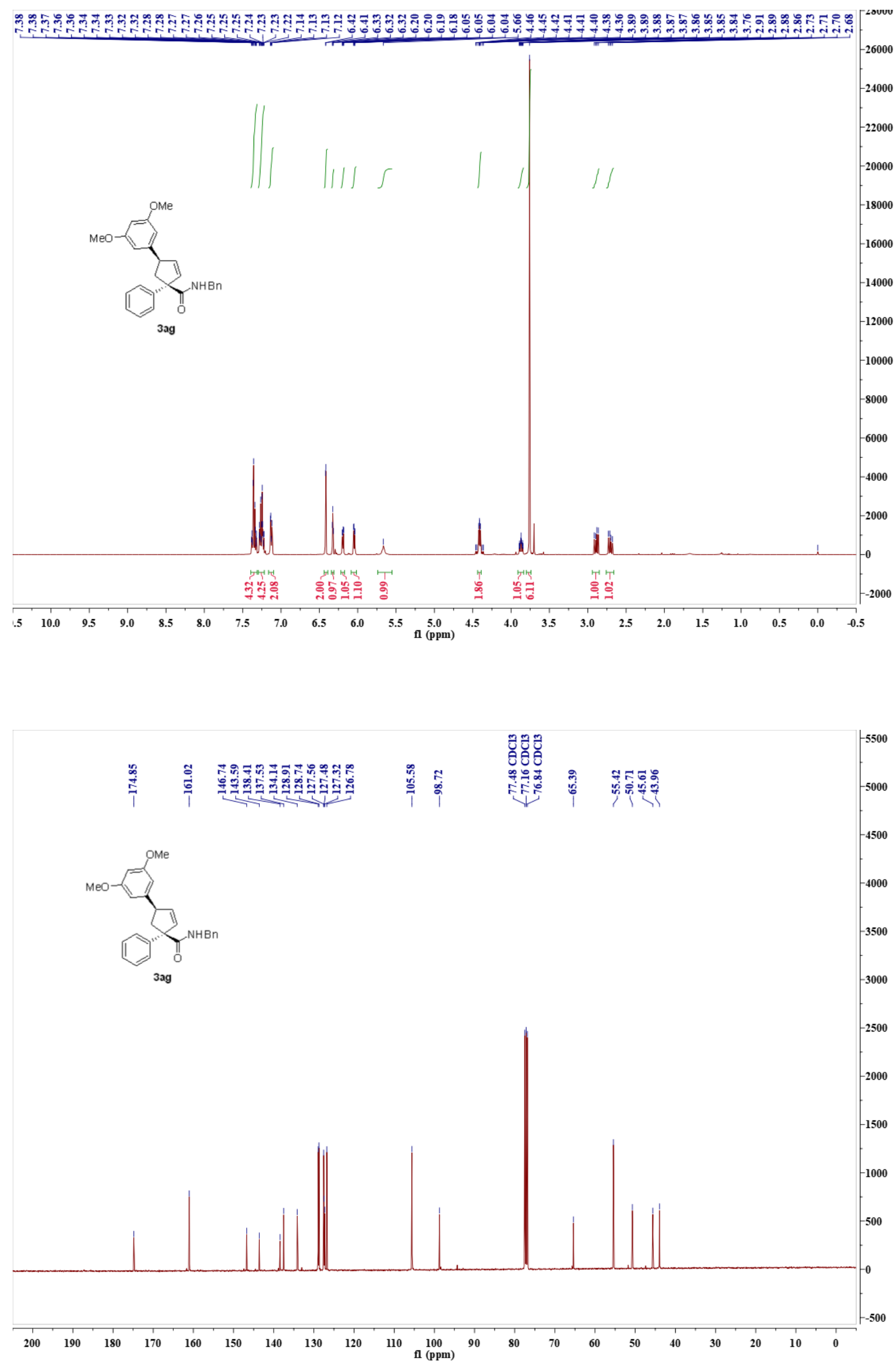

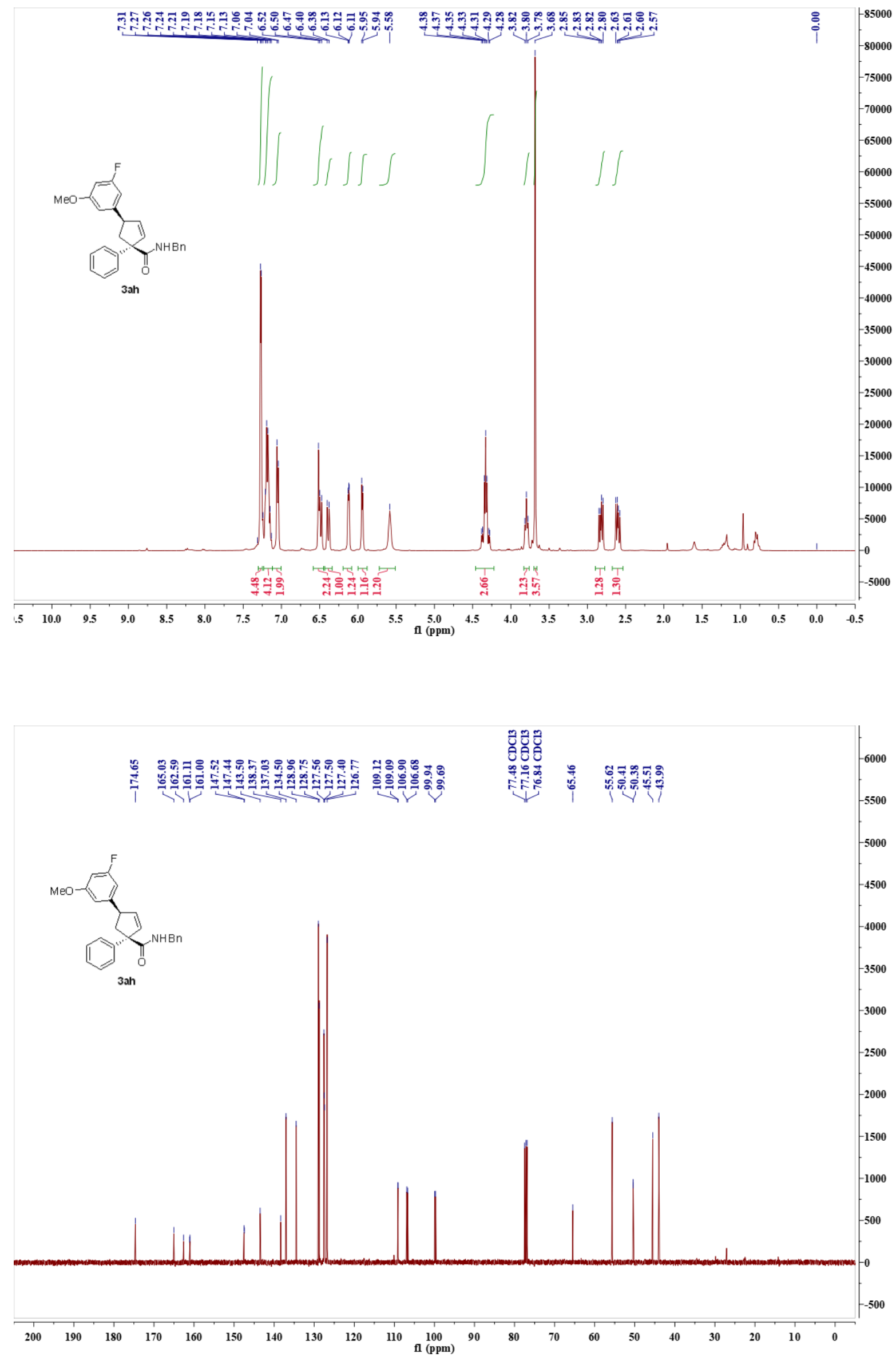

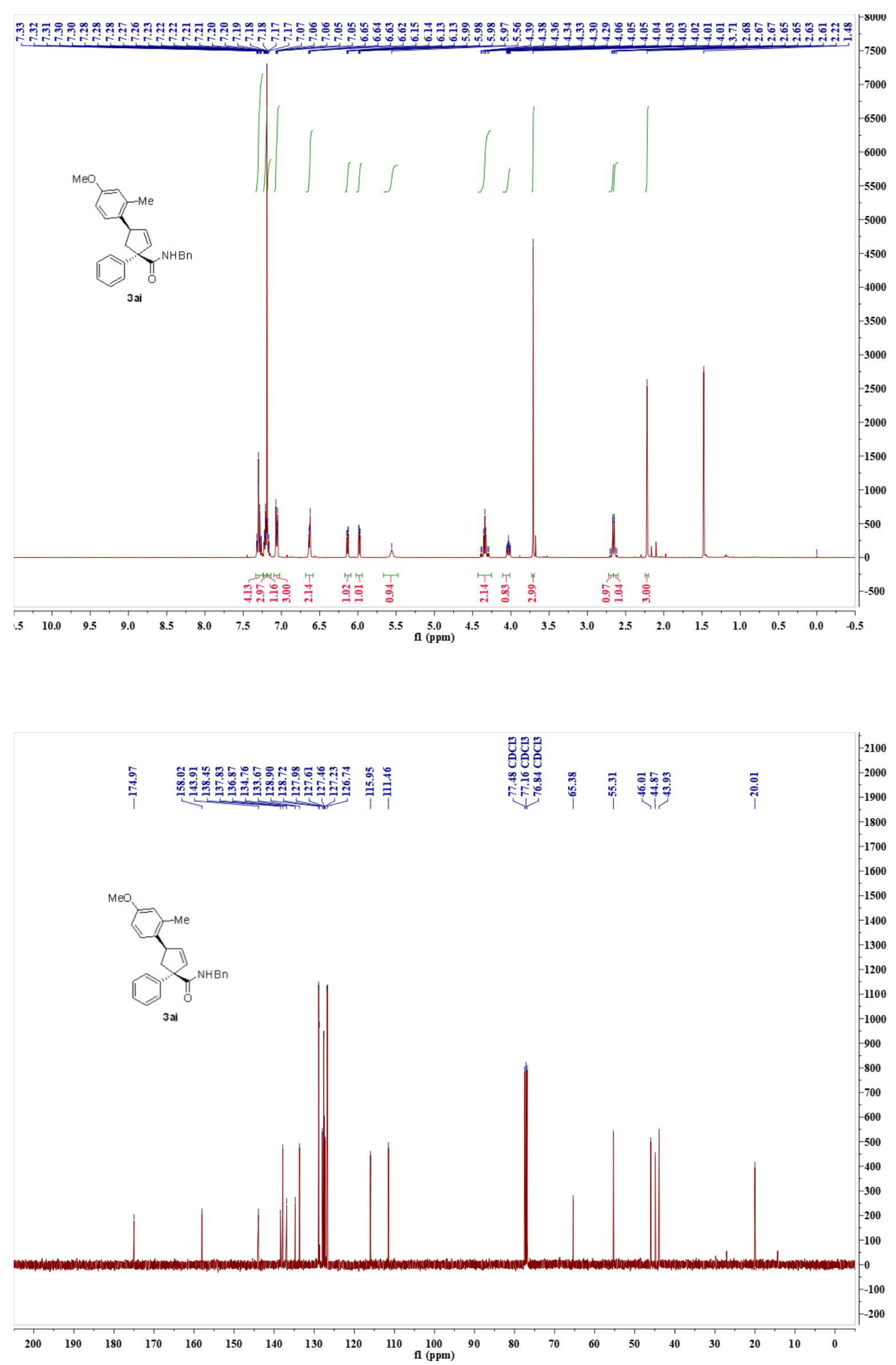

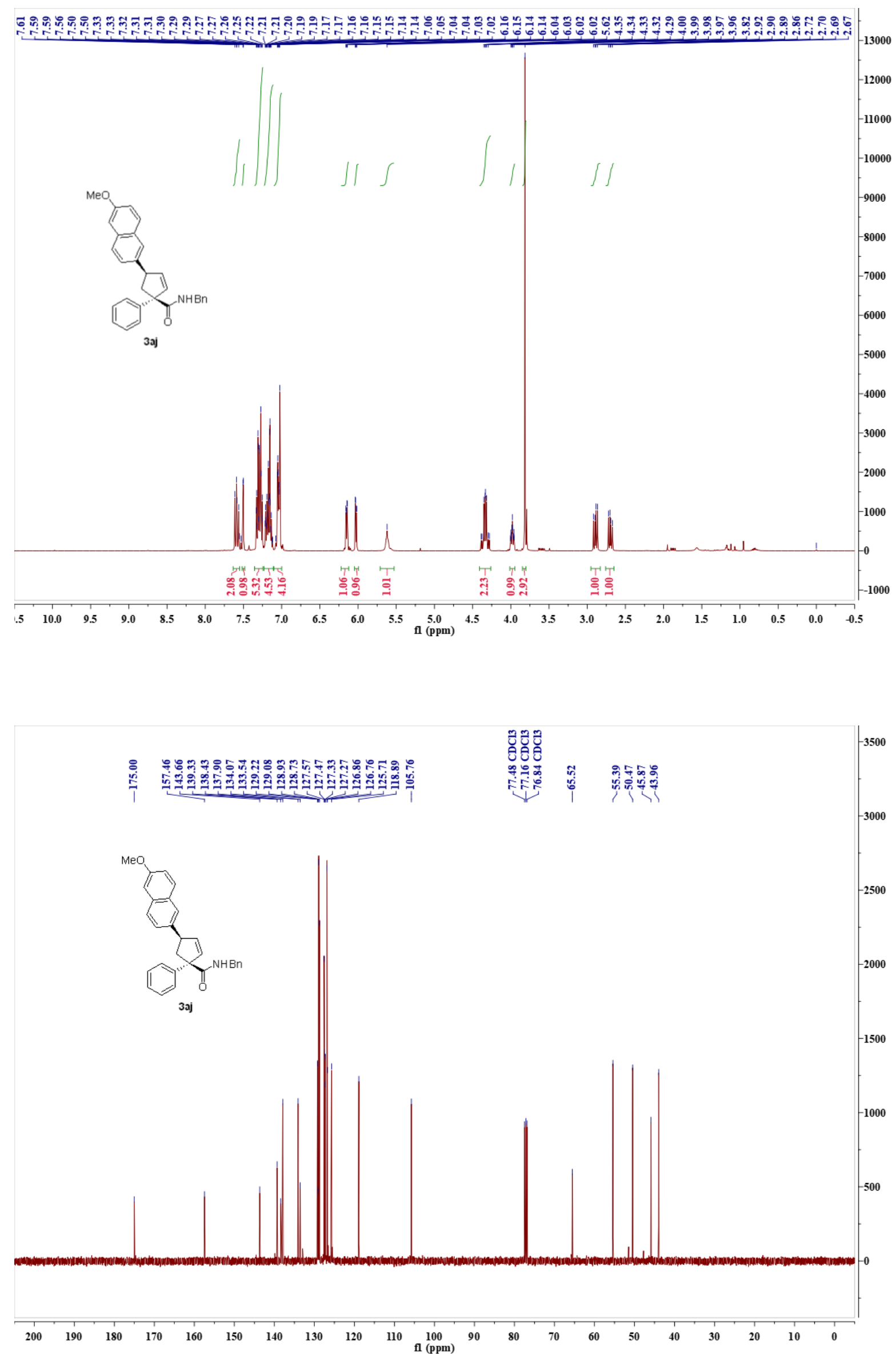

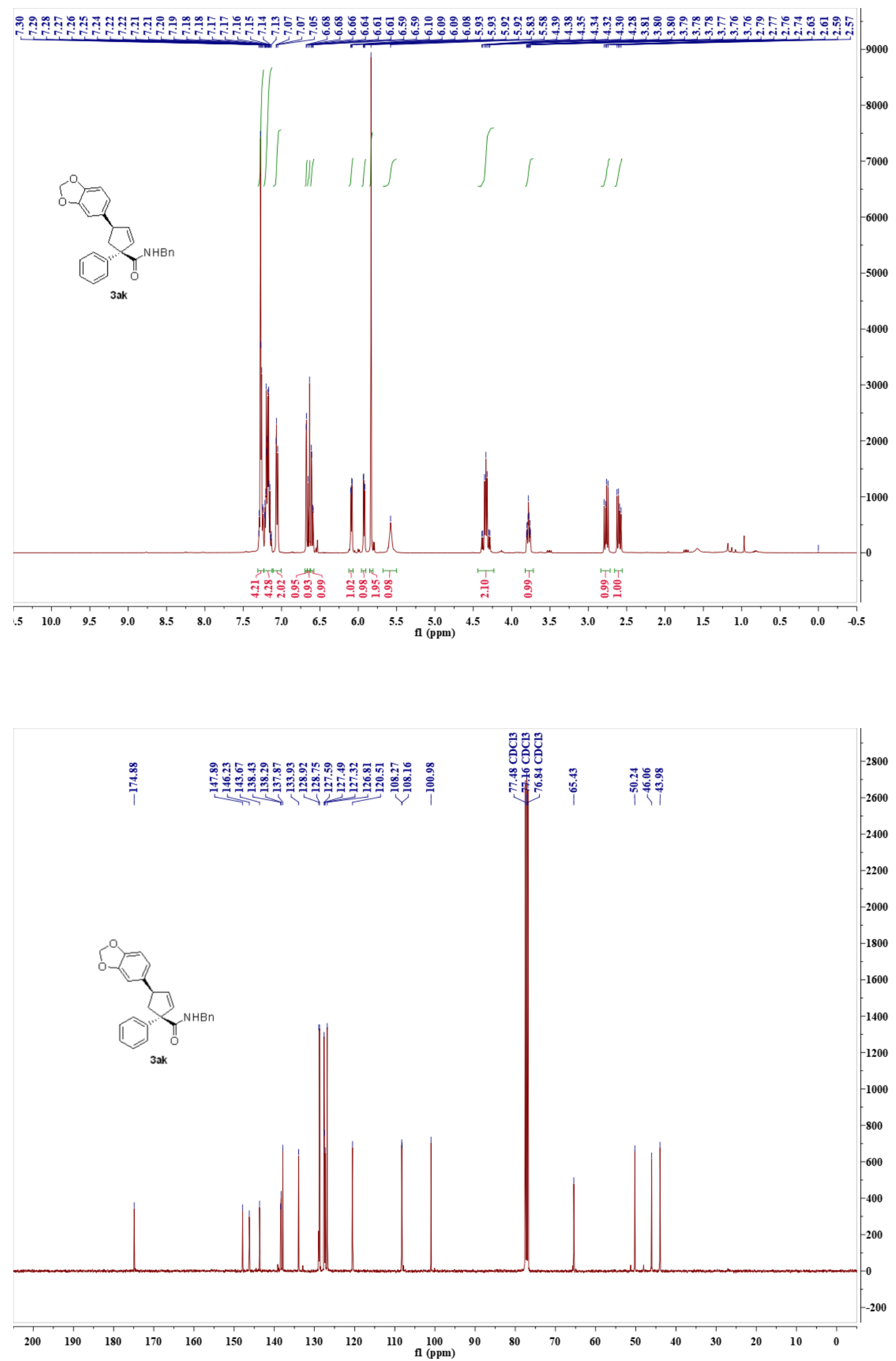

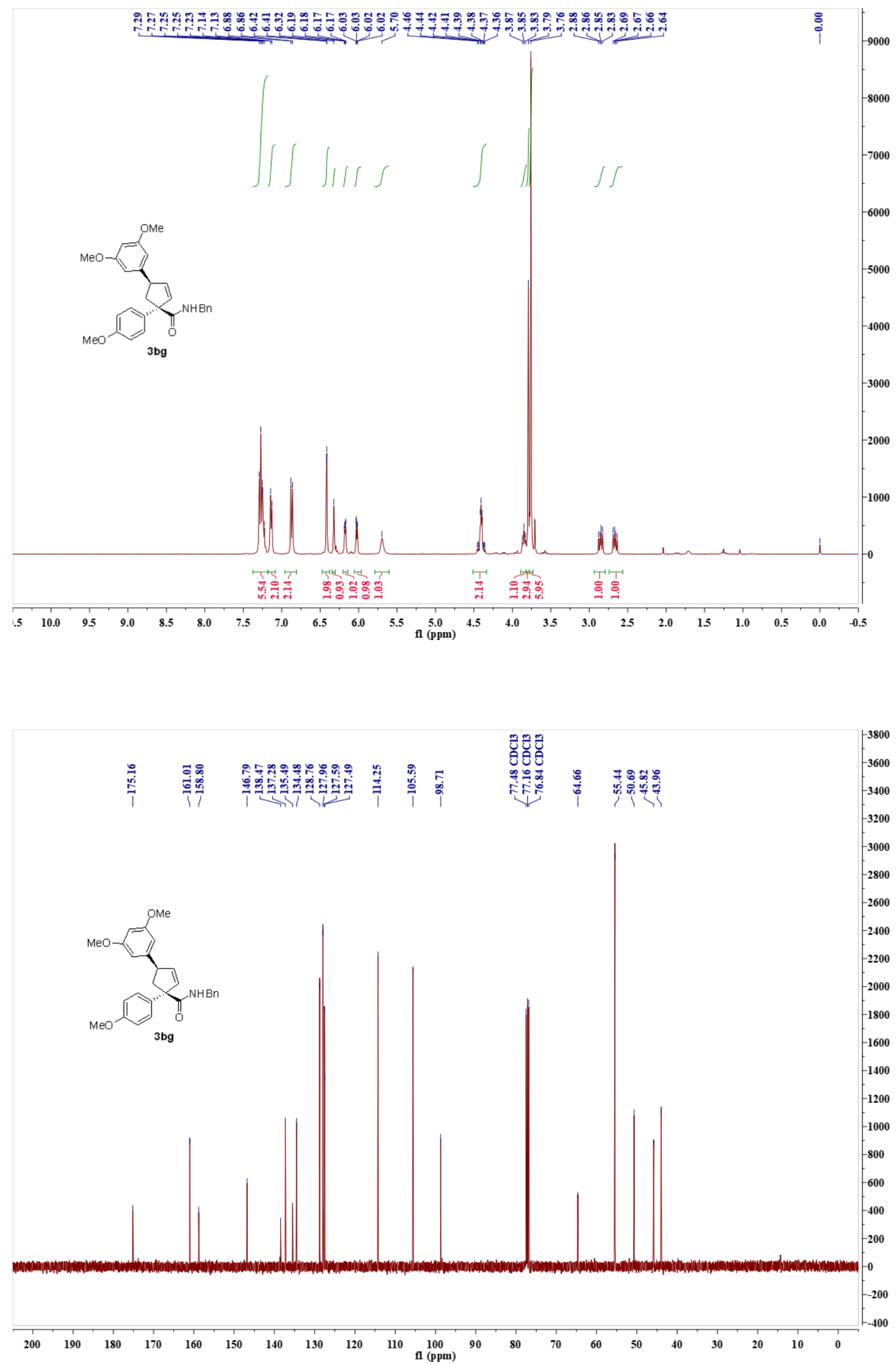

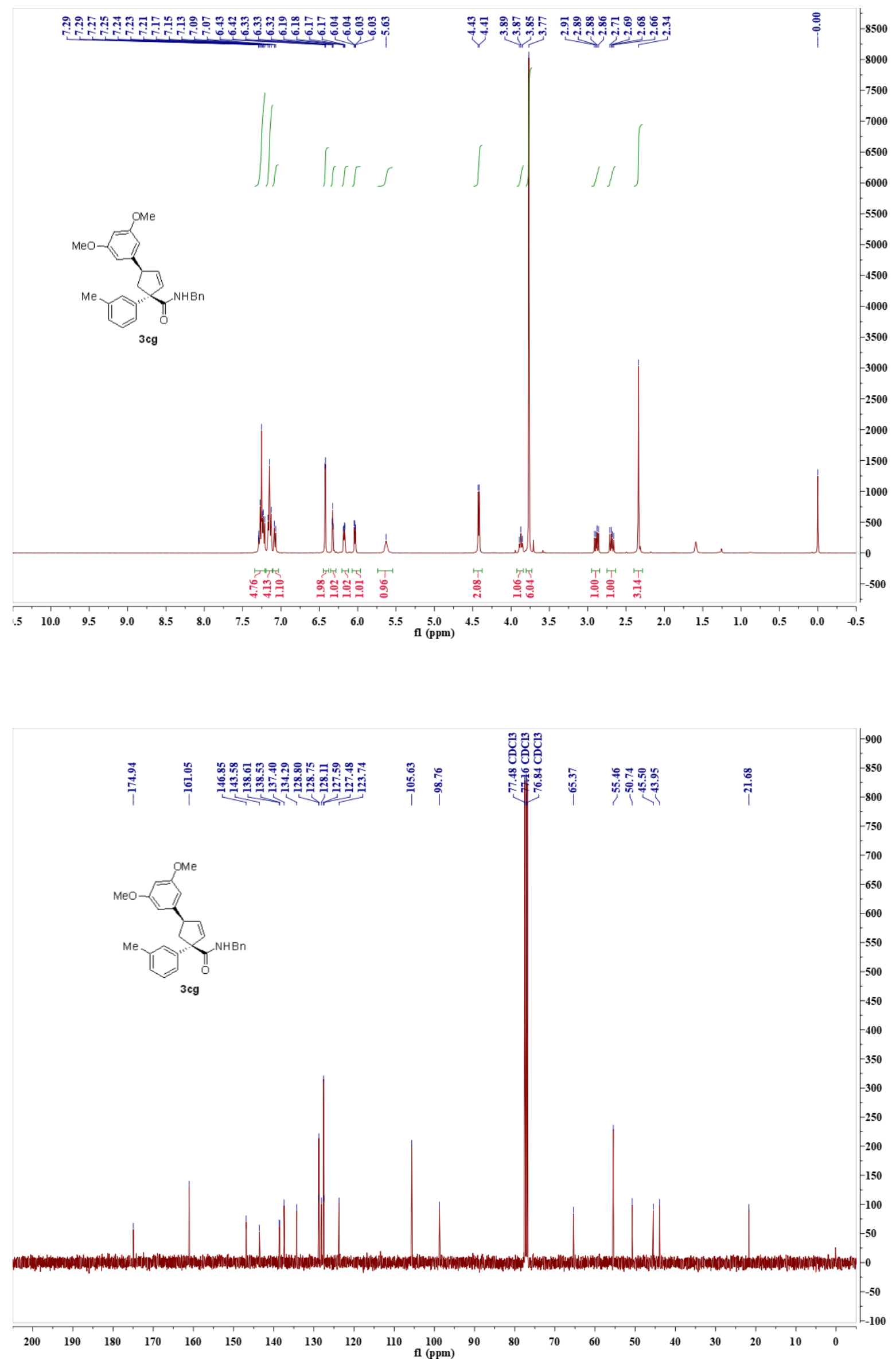

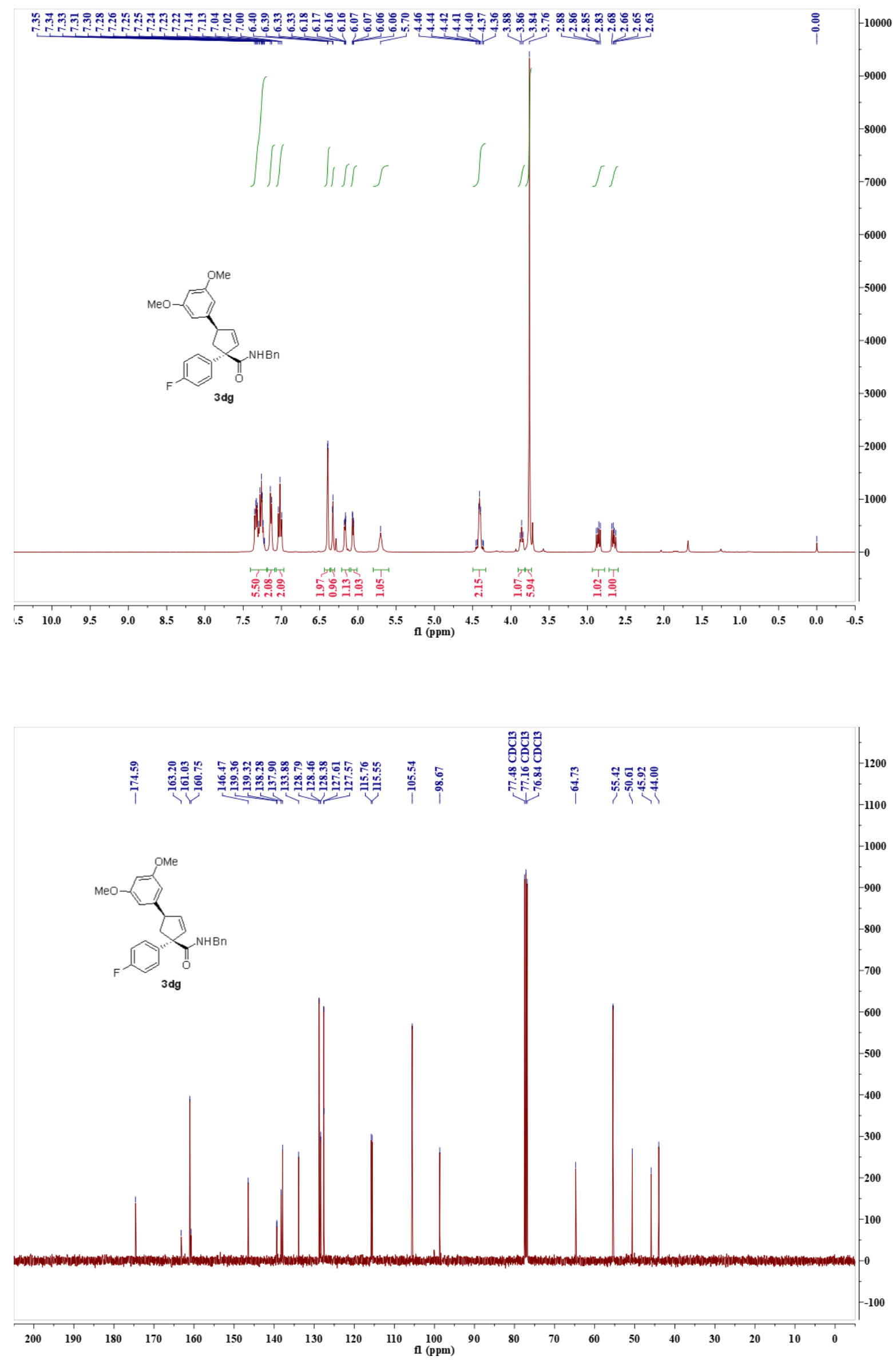

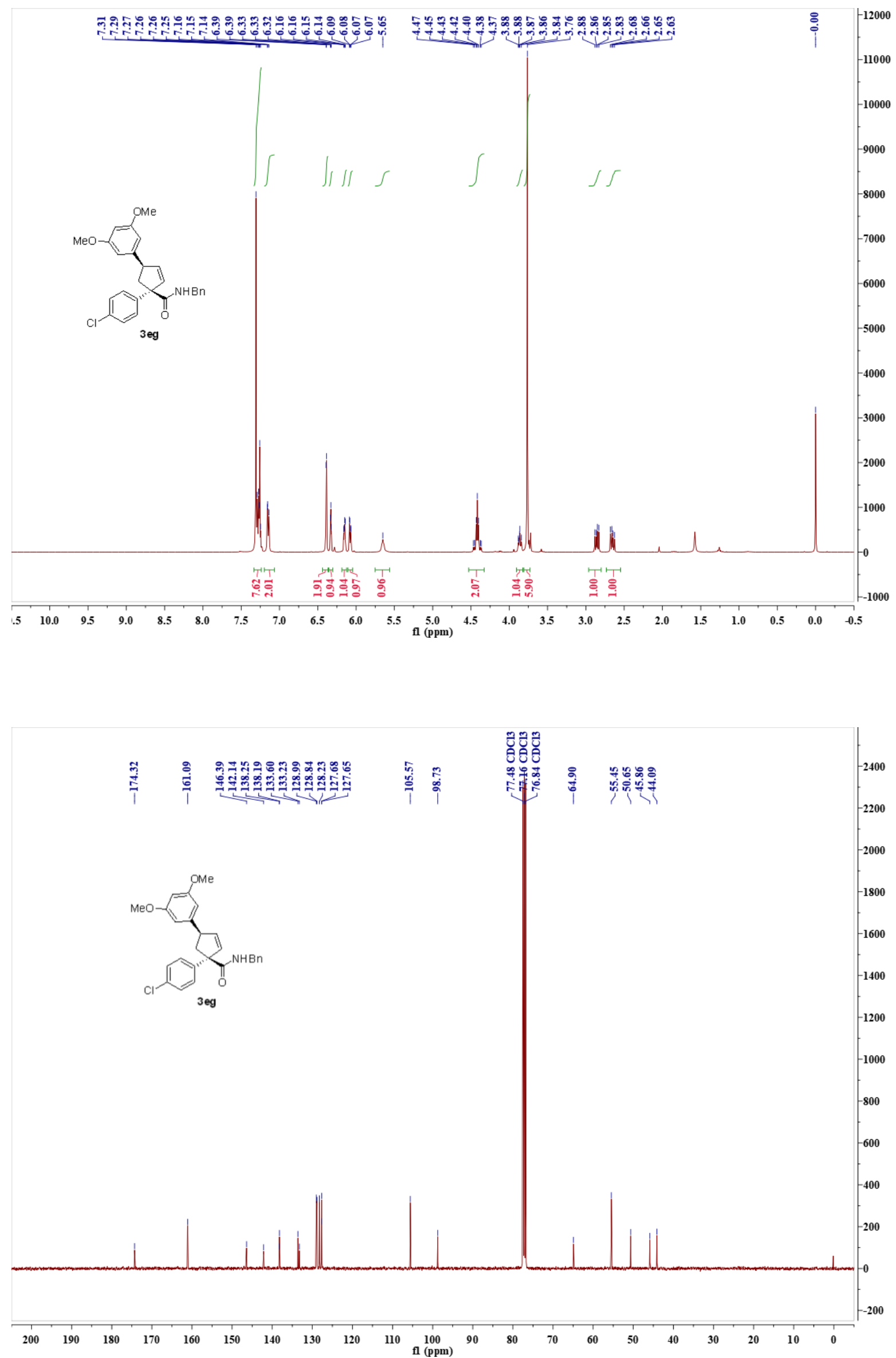

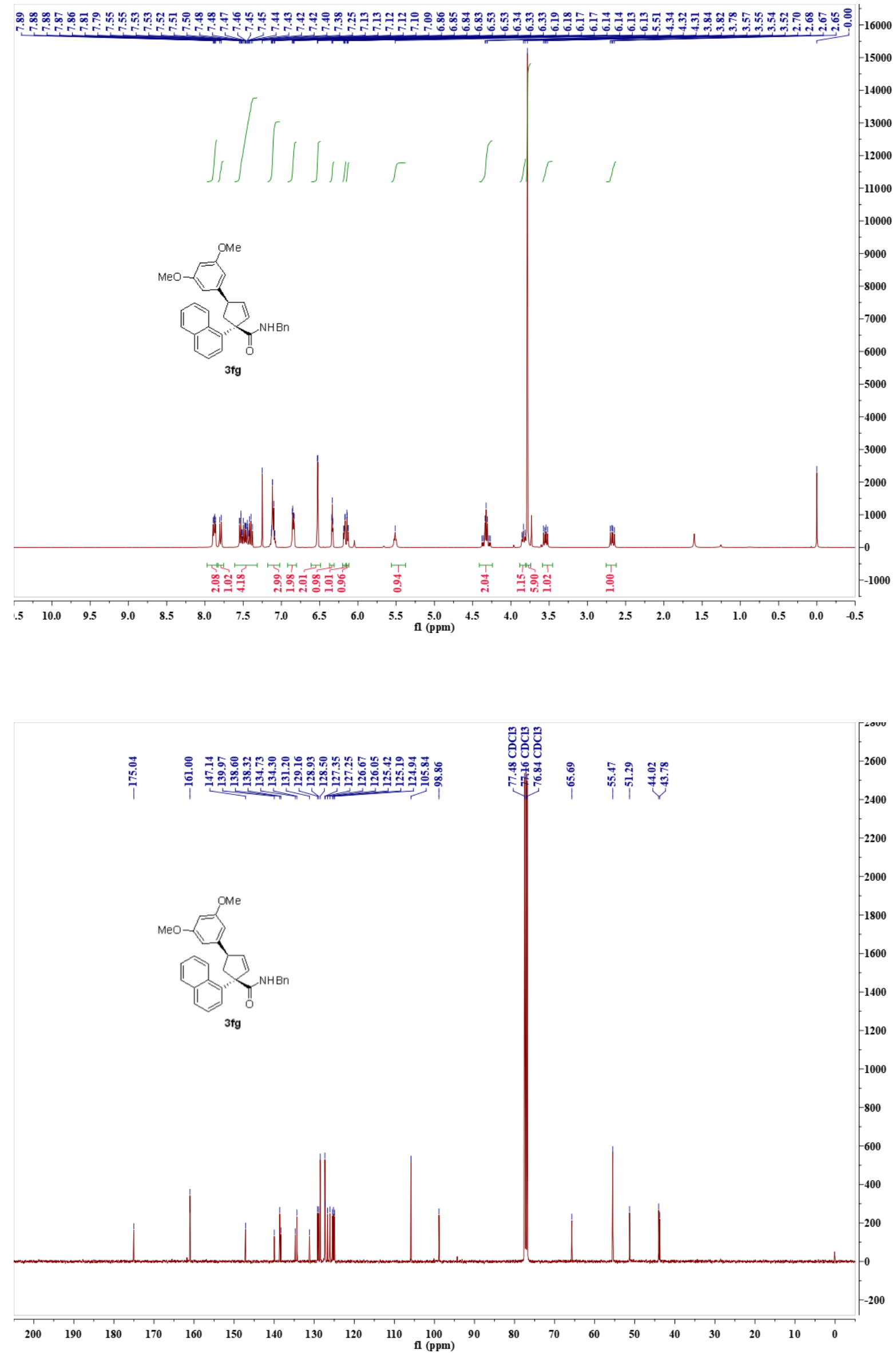

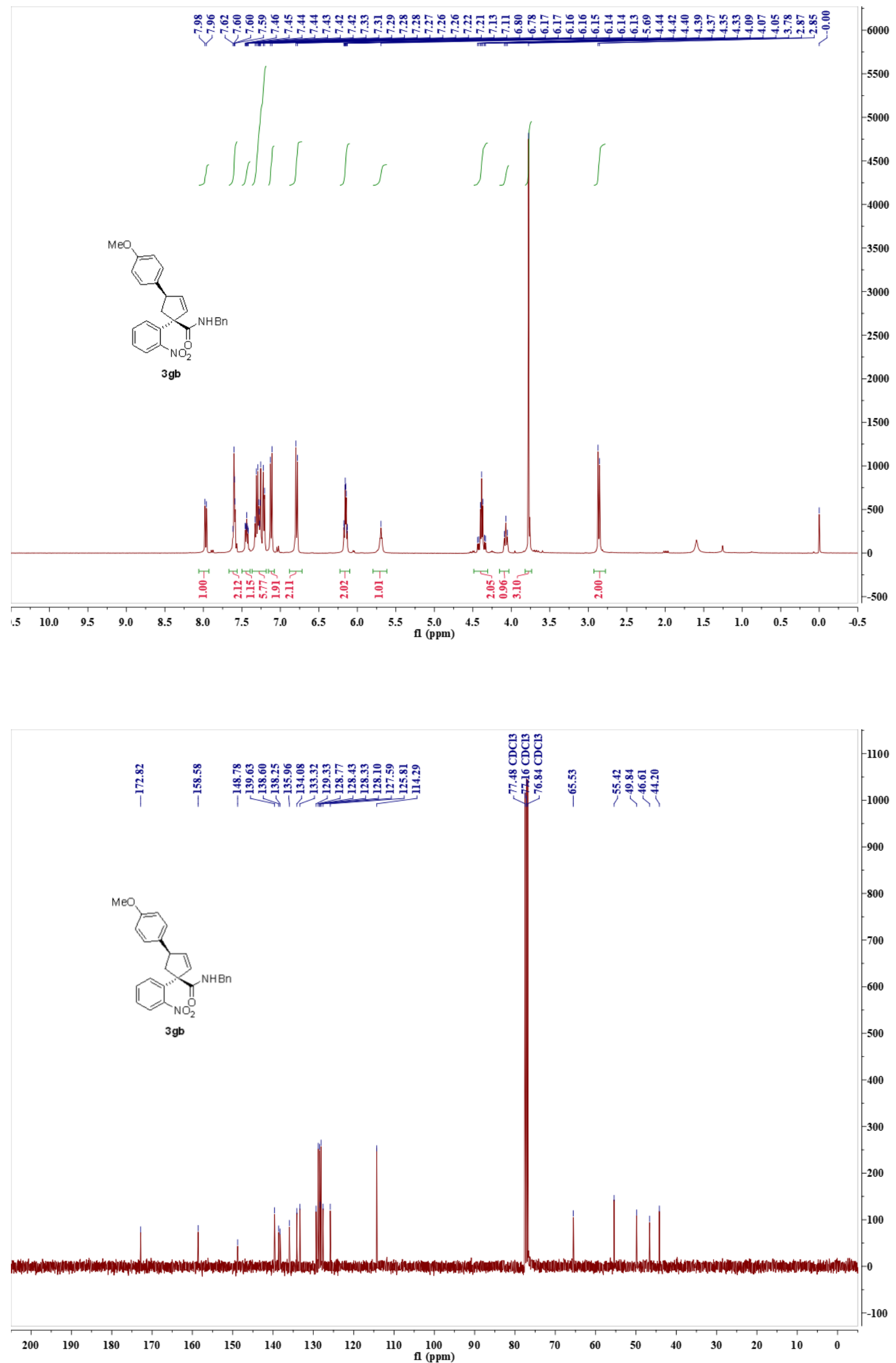

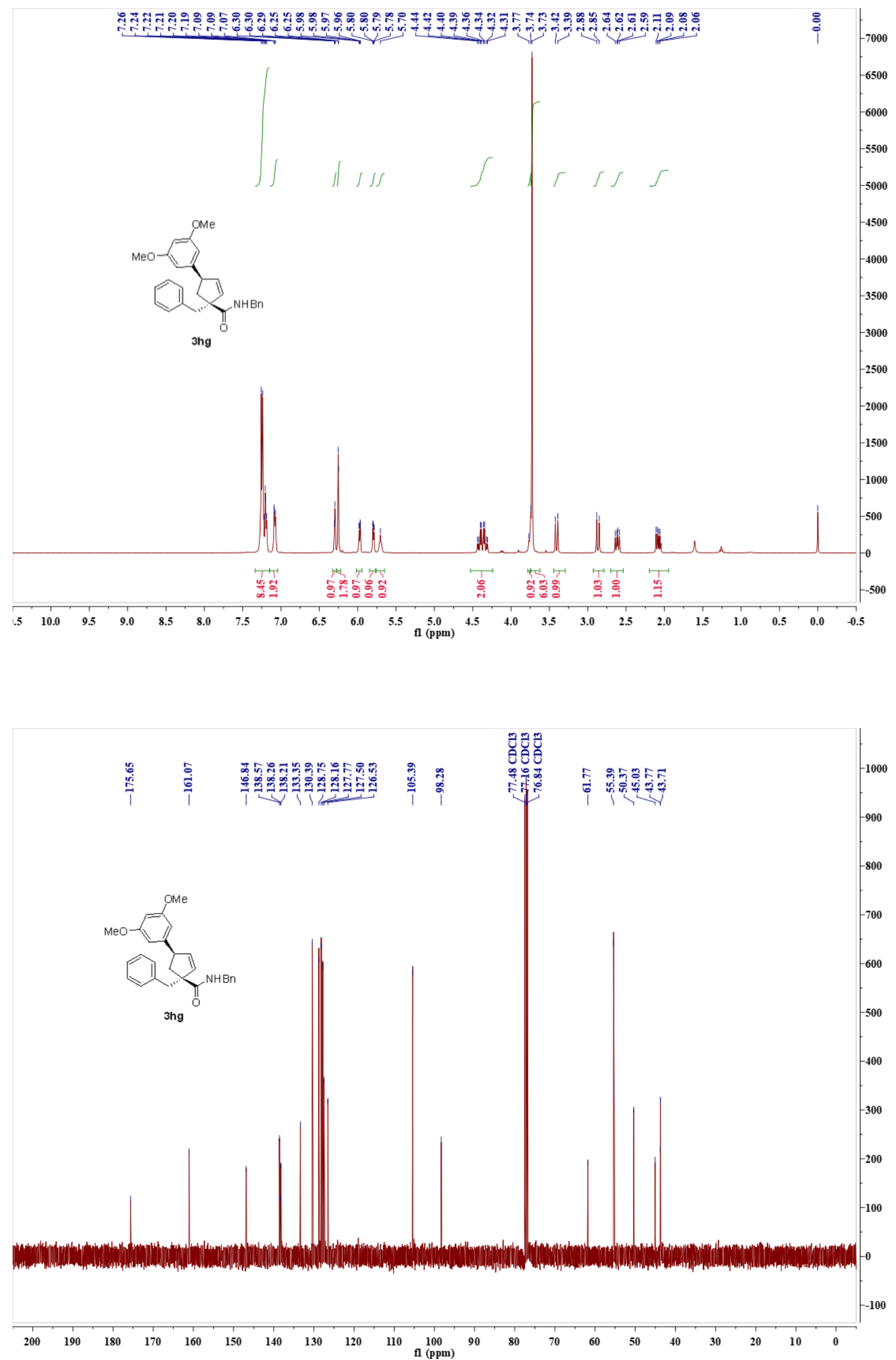

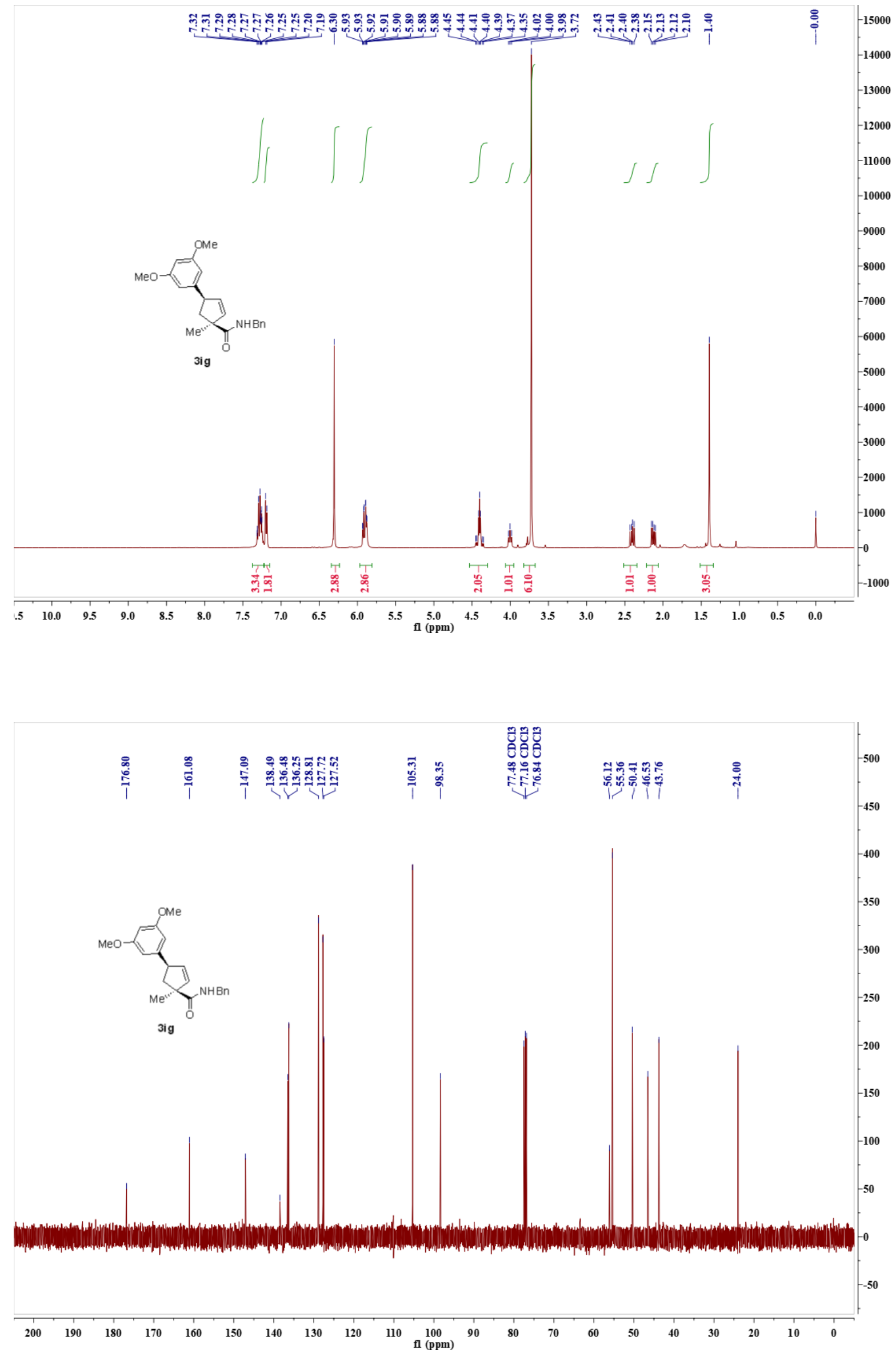

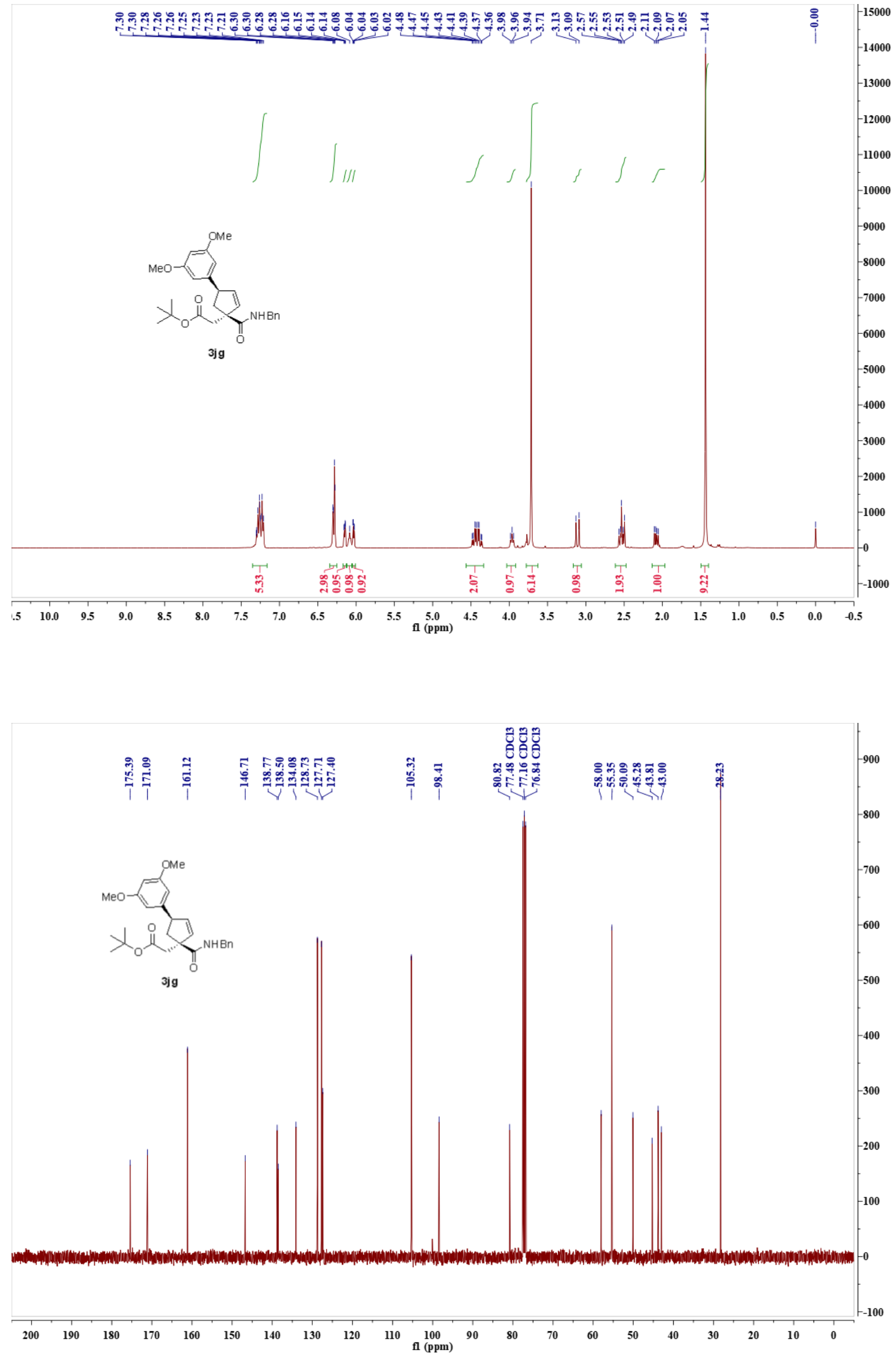

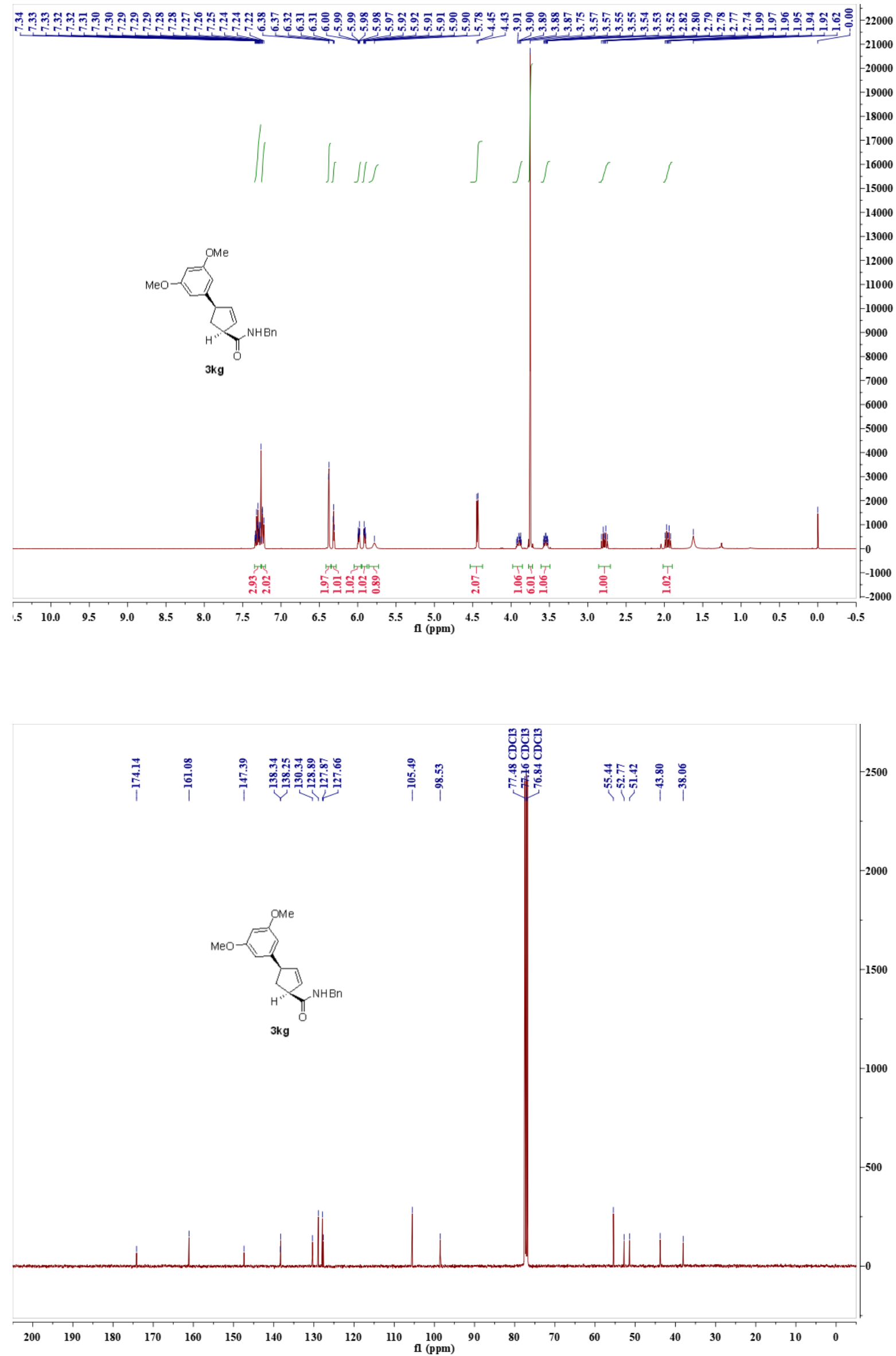

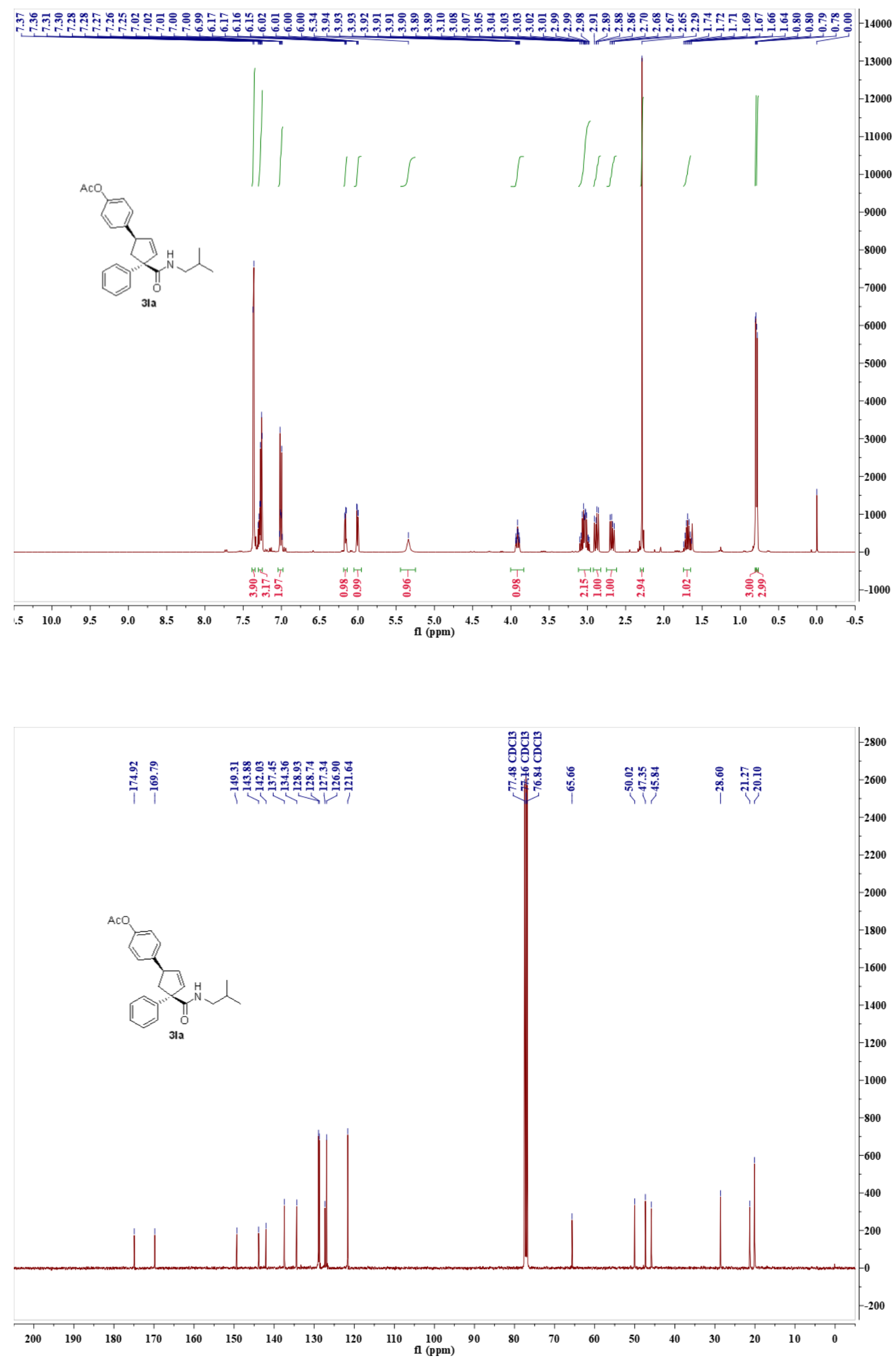

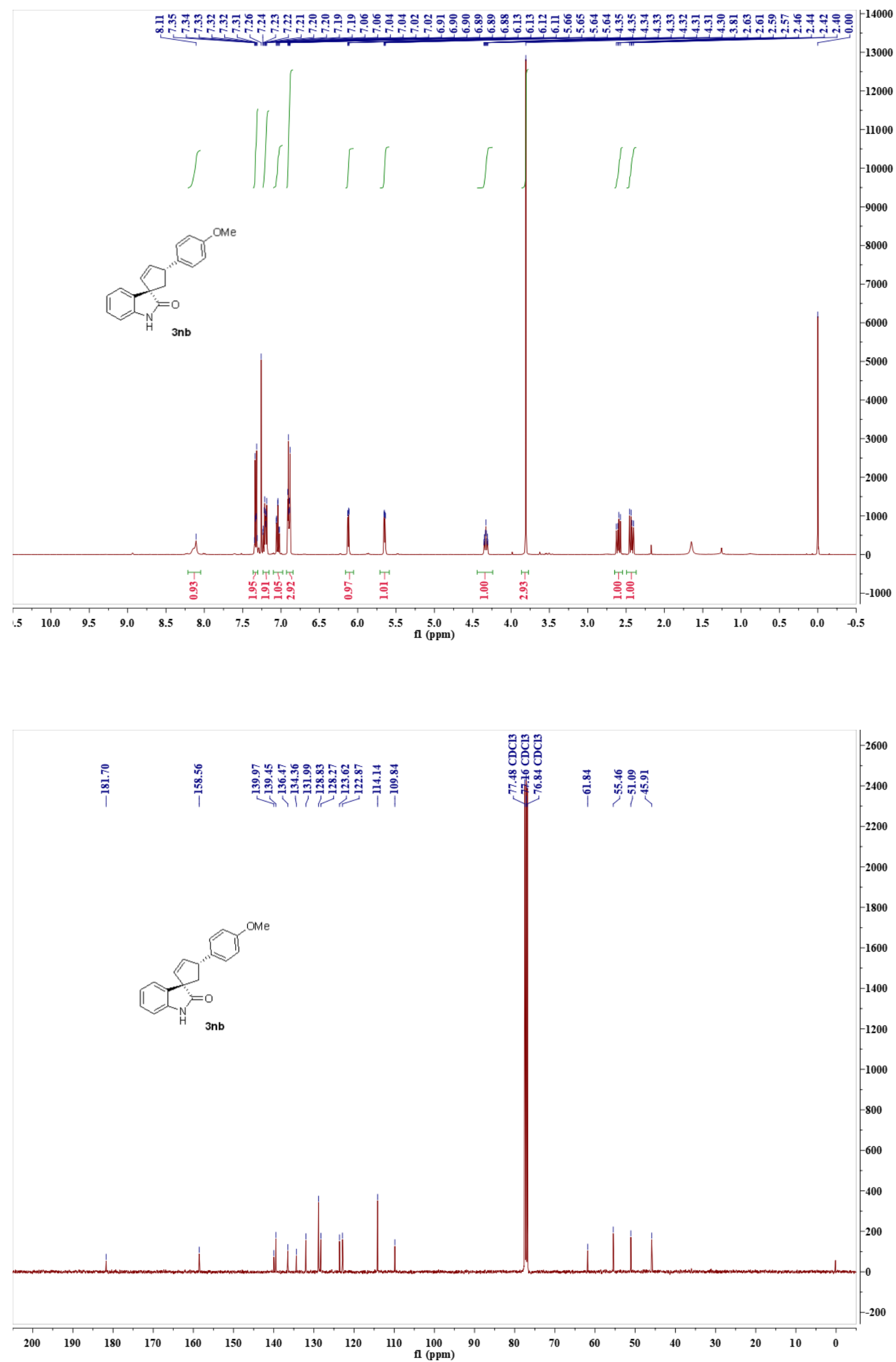

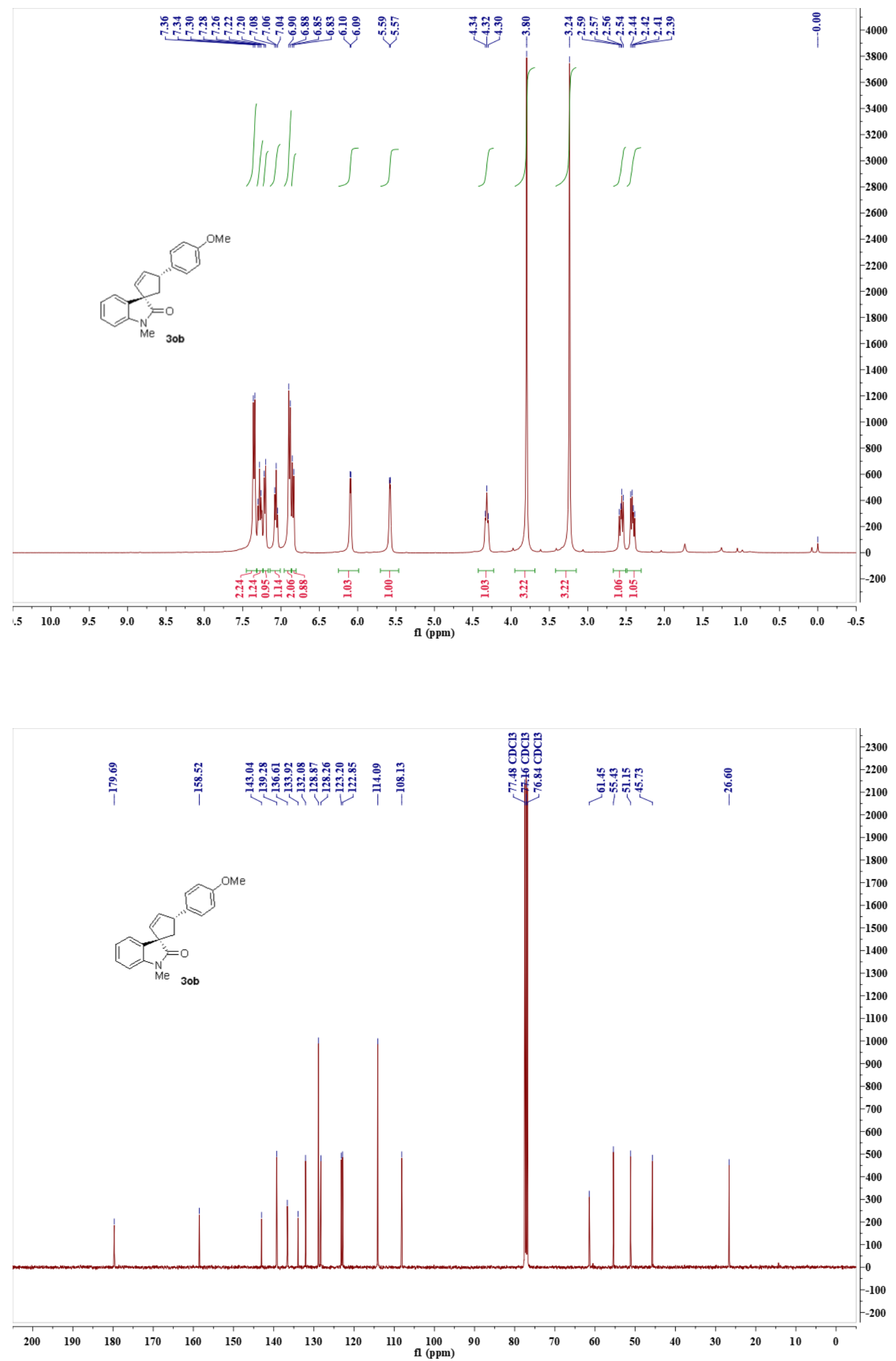

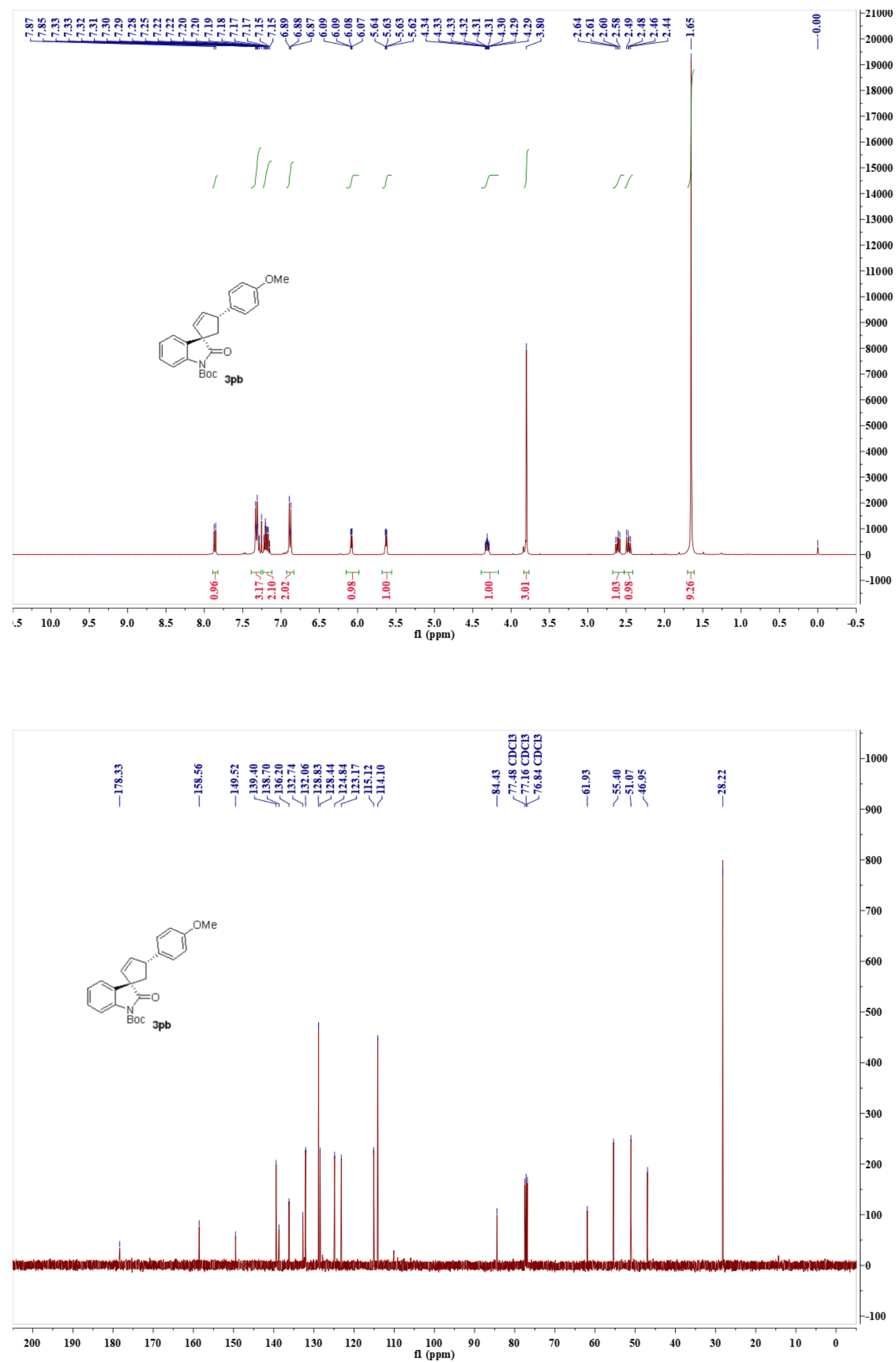

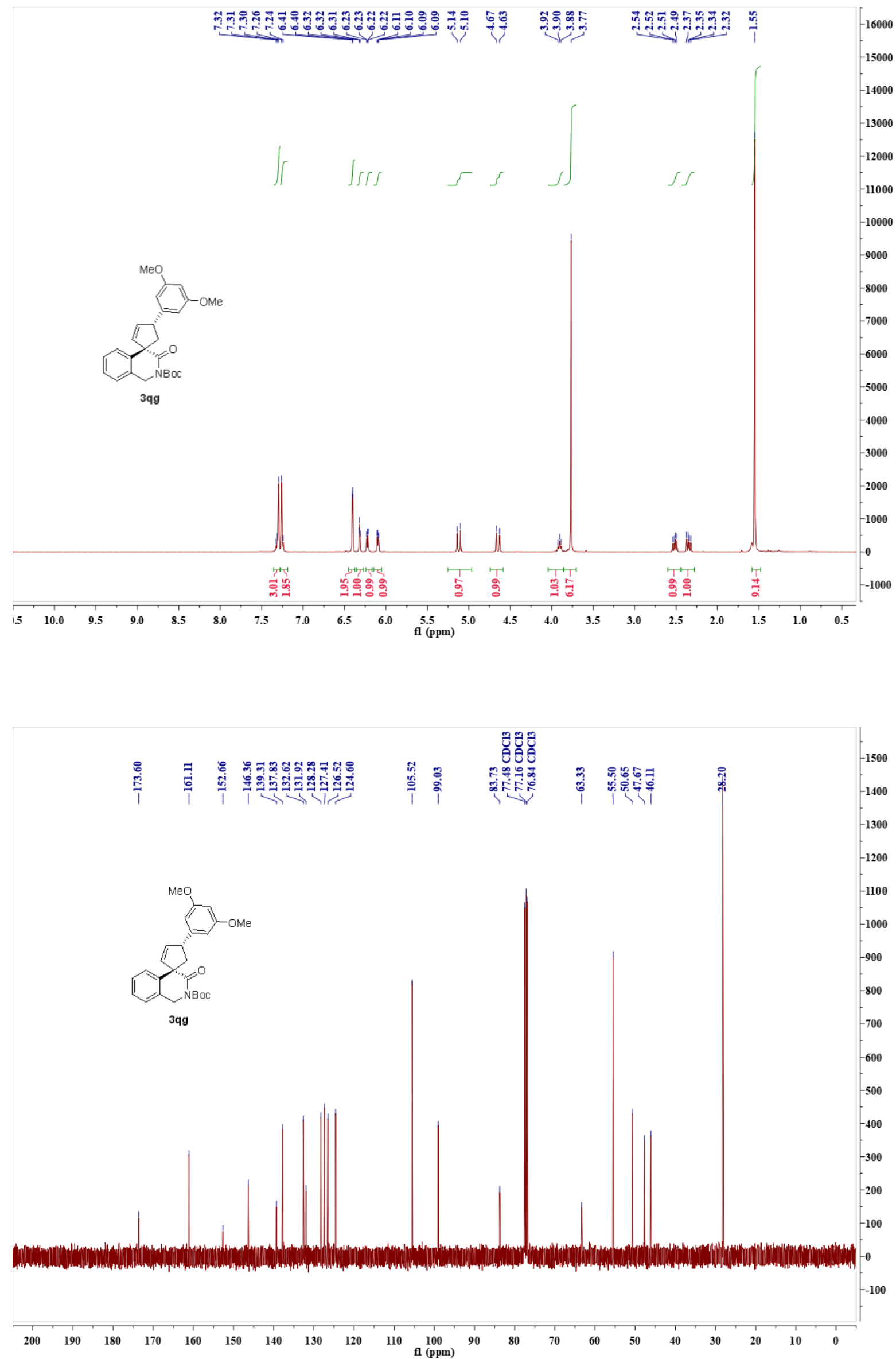

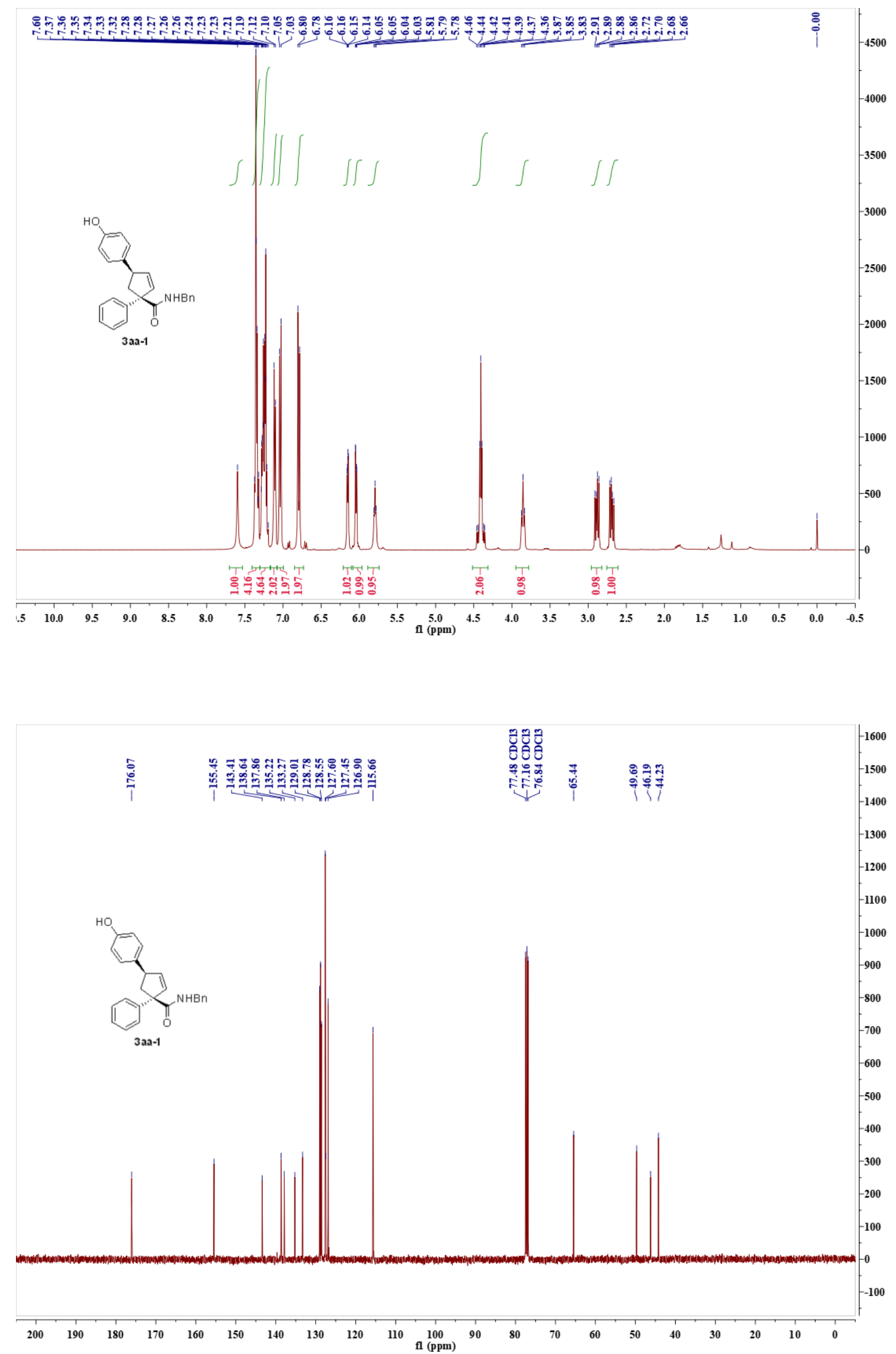

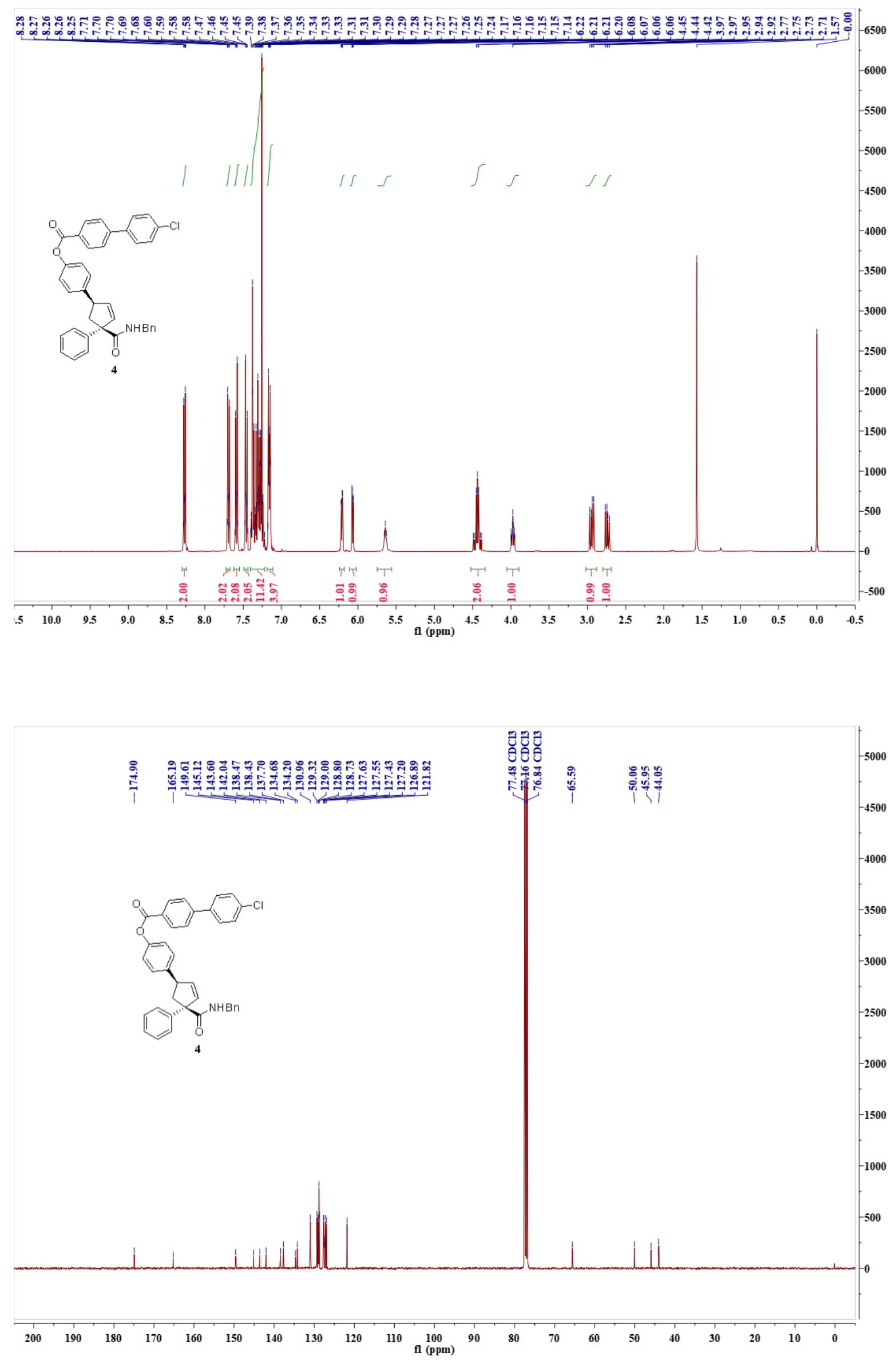

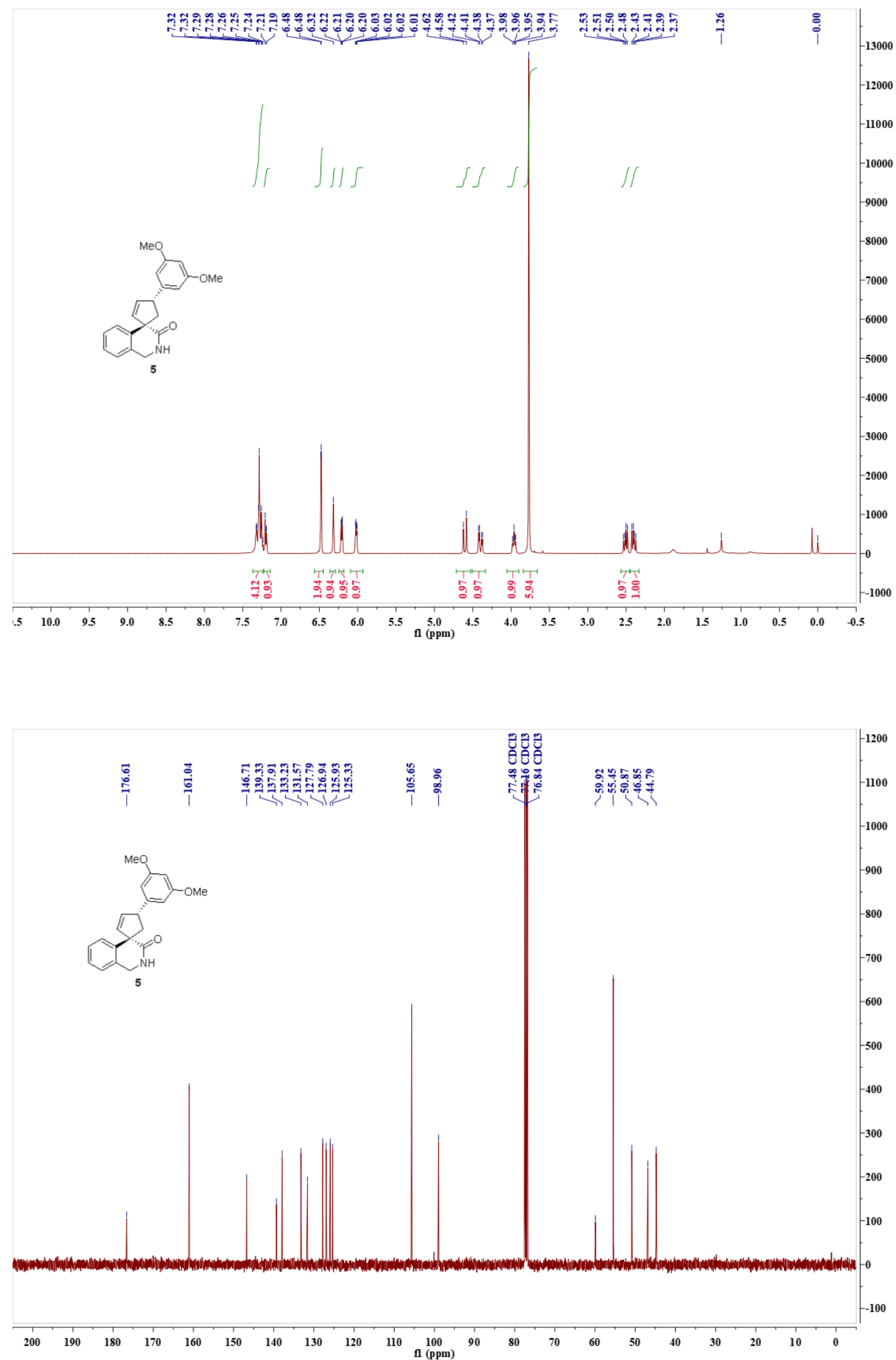

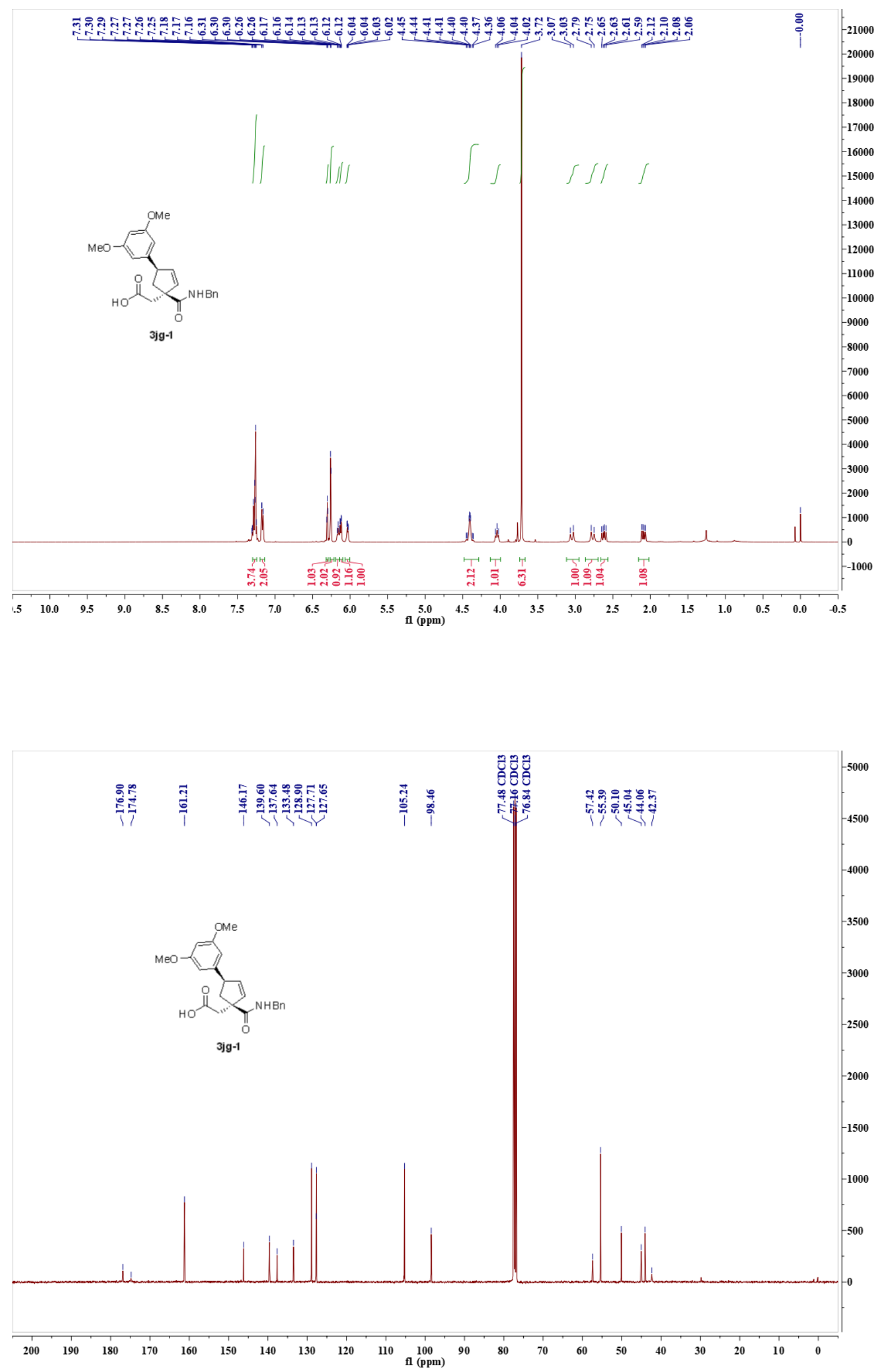

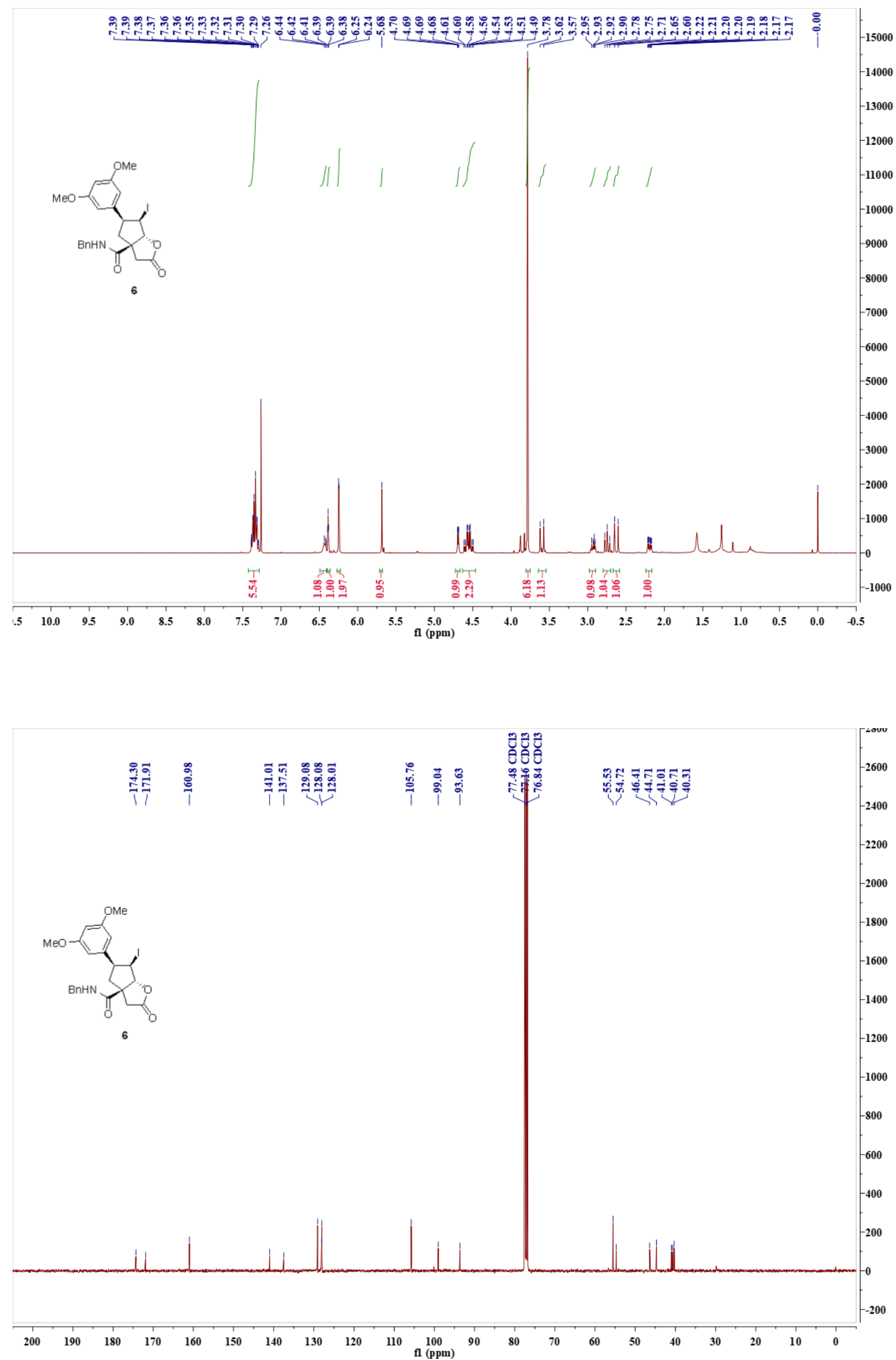

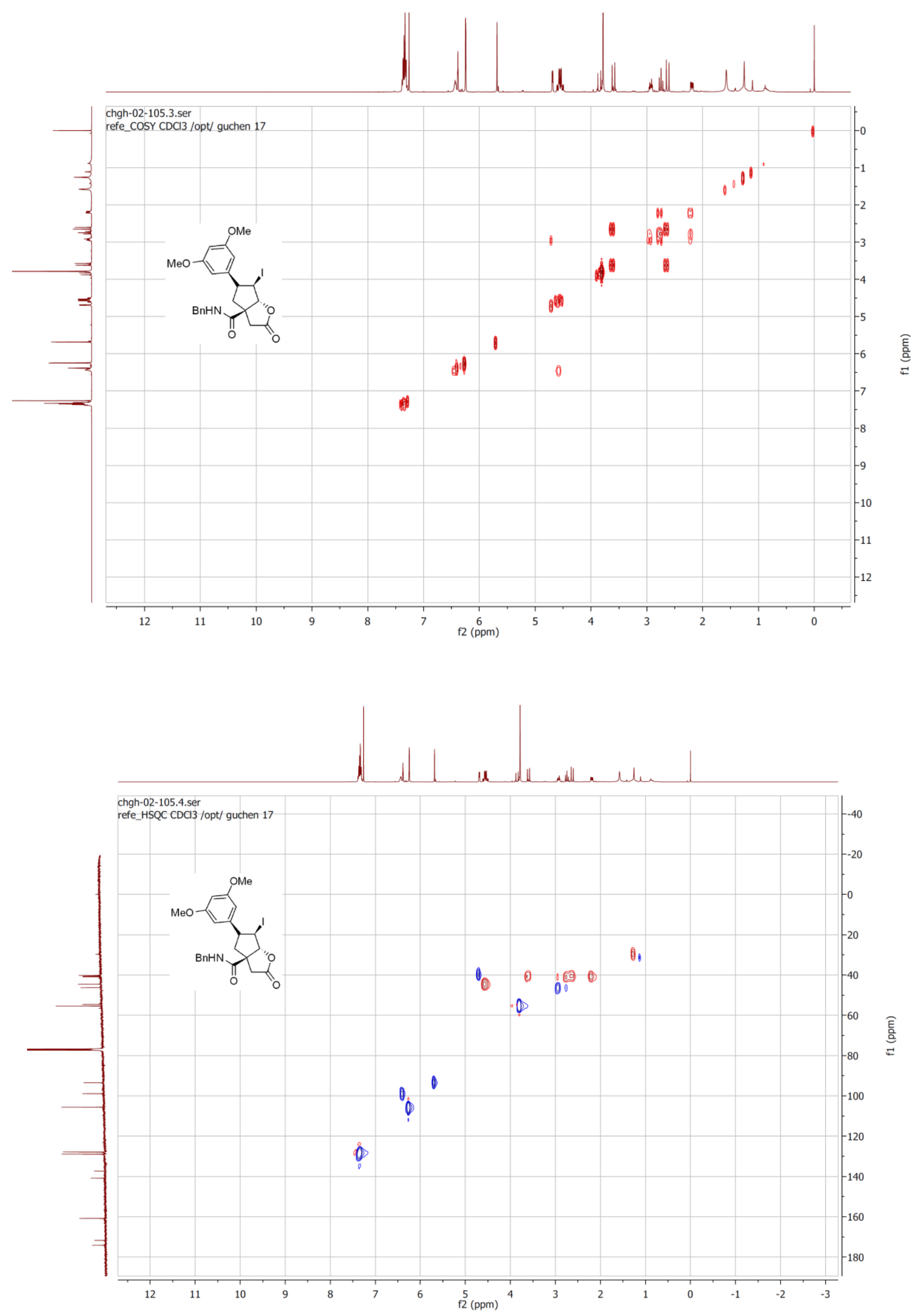

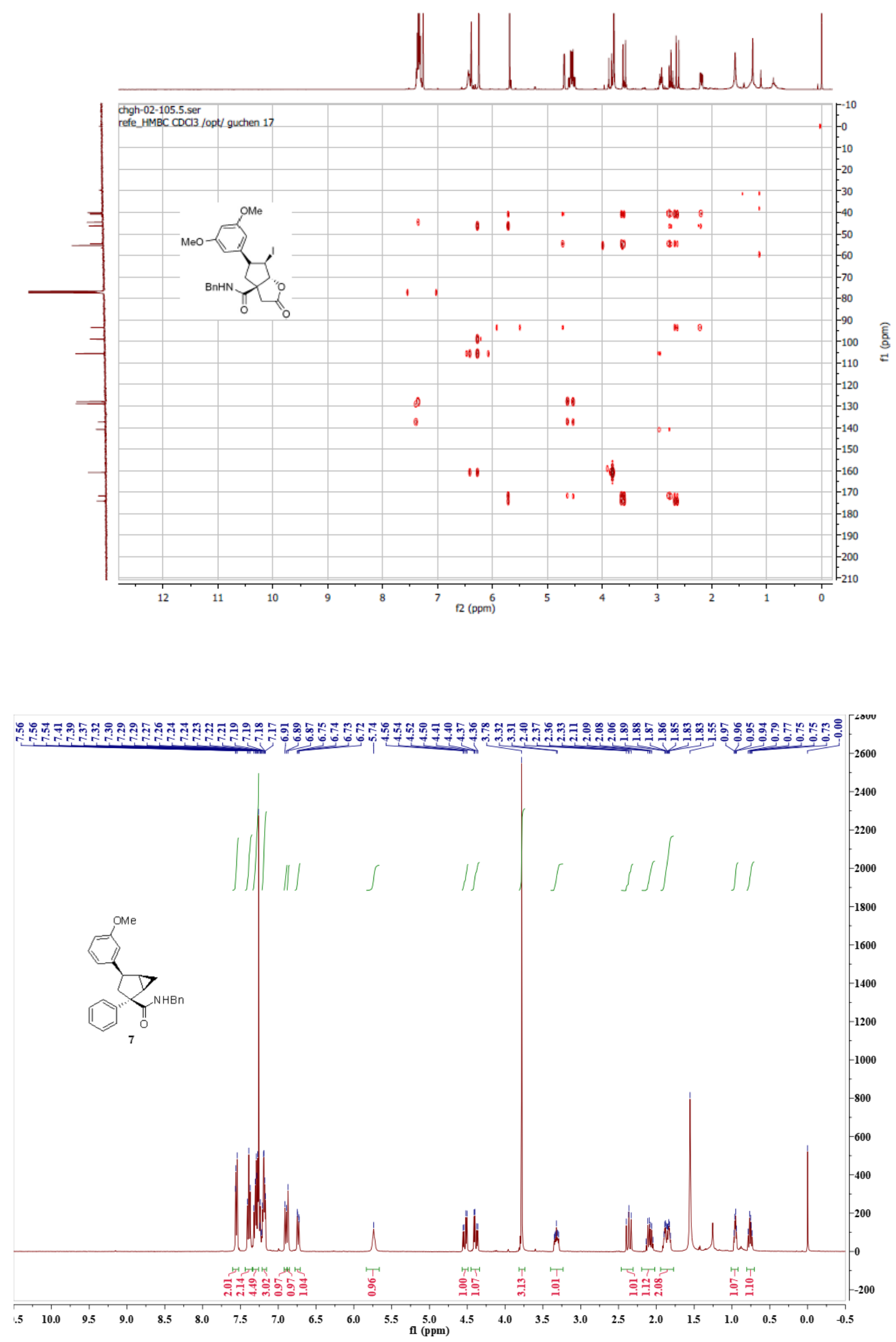

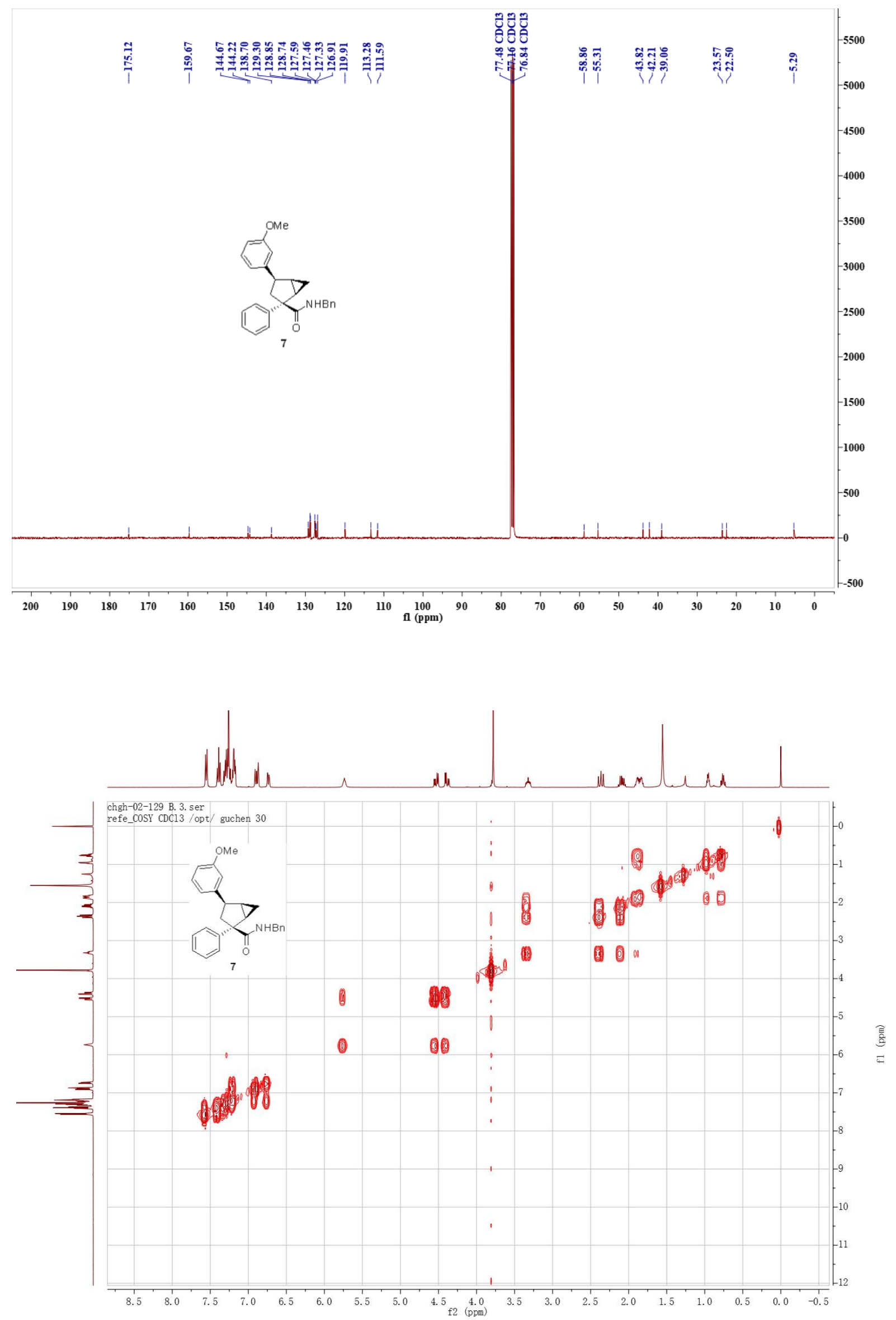

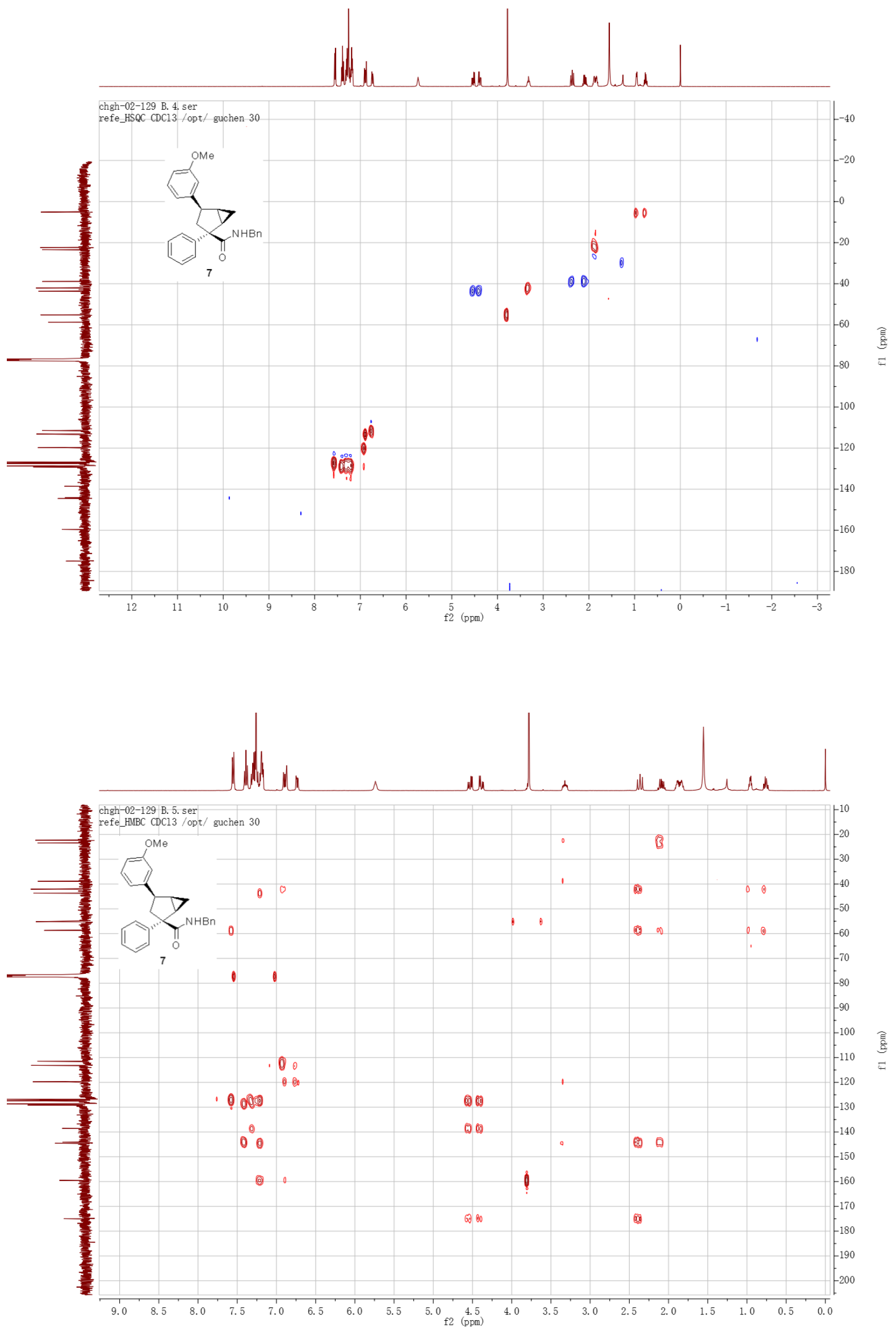


\section{Copies of SFC traces}

\begin{tabular}{|l|l|l|l|l|}
\hline Co-Solvent \% & Total Flow & Column & Co-Solvent & Back Pressure \\
\hline 15 & 4 & OJ-H & MeOH & 150 \\
\hline
\end{tabular}
\begin{tabular}{|l|l|l|l|}
\hline Peak \# & Ret. Time & Area & Area $\%$ \\
\hline 1 & 6.07 min & 11010.1228 & 50.0226 \\
\hline 2 & 13.17 min & 11000.1622 & 49.9774 \\
\hline
\end{tabular}
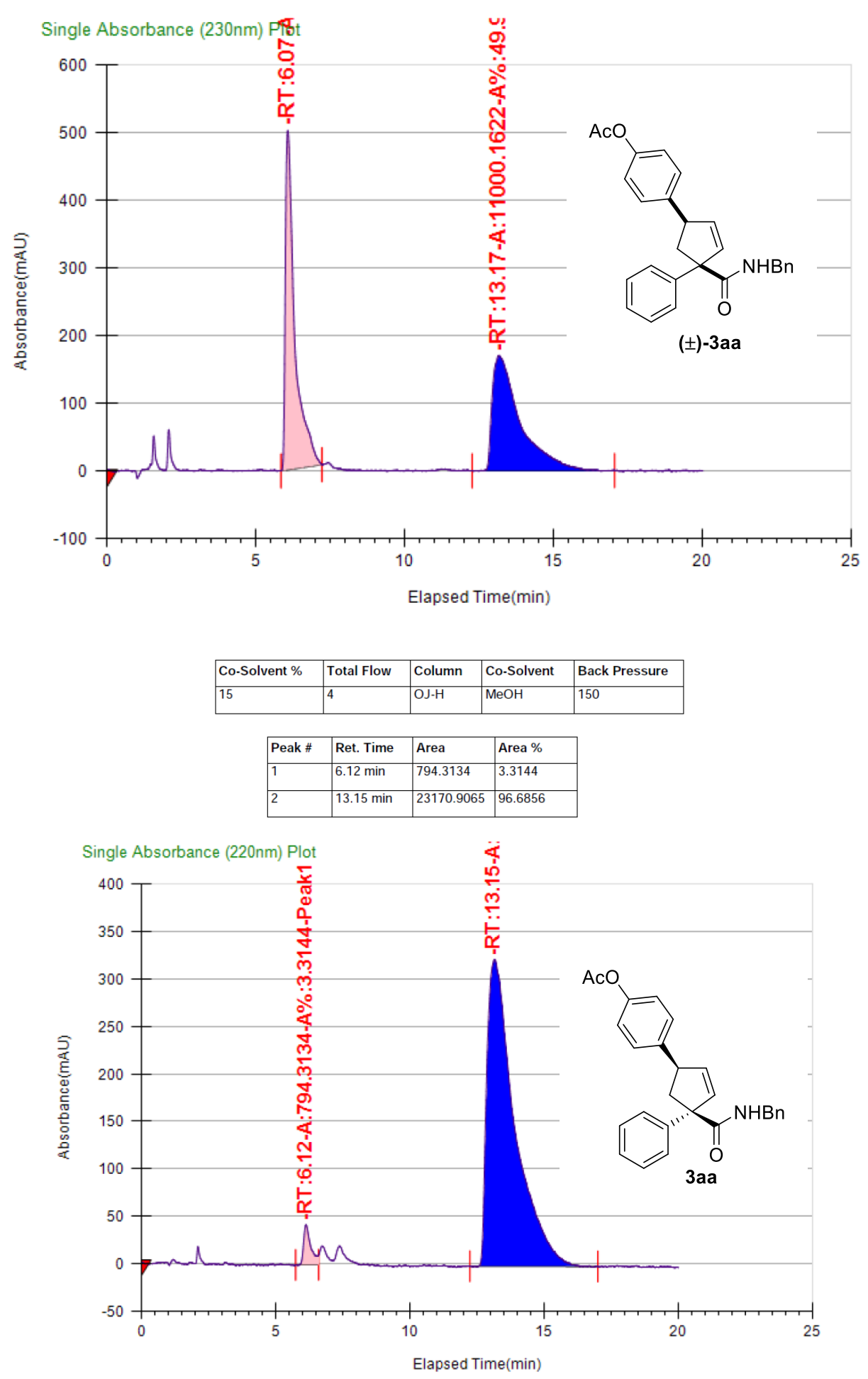


\begin{tabular}{|c|c|c|c|c|}
\hline Co-Solvent \% & Total Flow & Column & Co-Solvent & Back Pressure \\
\hline 8 & 4 & $\mathrm{OJ}-\mathrm{H}$ & $\mathrm{MeOH}$ & 150 \\
\hline Peak \# & Ret. Time & Area & Area \% & \\
\hline 1 & $16.84 \mathrm{~min}$ & 18706.2845 & \begin{tabular}{|l|l|}
5 & 49.4303
\end{tabular} & \\
\hline 2 & $20.82 \mathrm{~min}$ & 19137.4956 & \begin{tabular}{l|l}
3 & 50.5697
\end{tabular} & \\
\hline
\end{tabular}
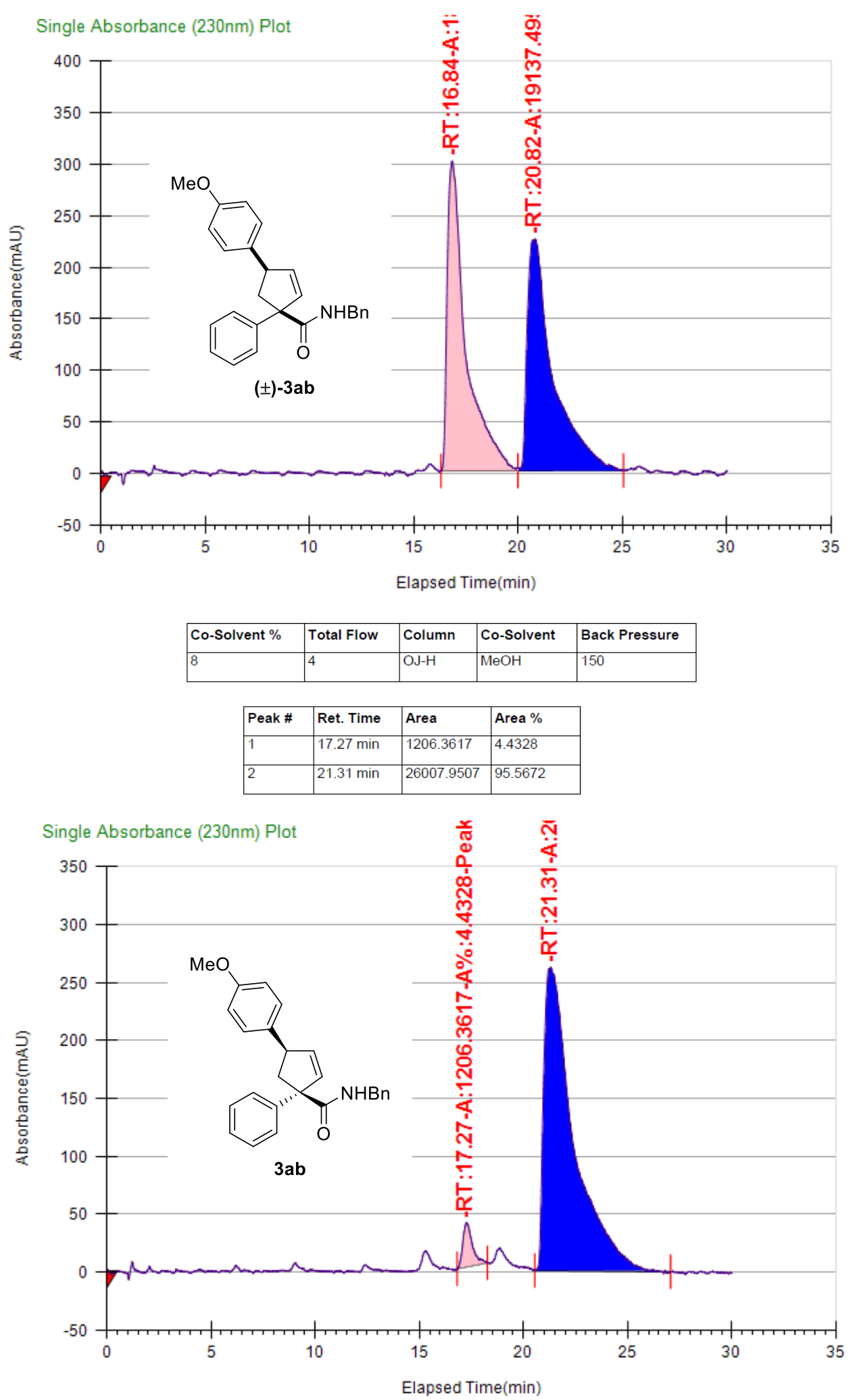


\begin{tabular}{|c|c|c|c|c|}
\hline Co-Solvent \% & Total Flow & Column & Co-Solvent & Back Pressure \\
\hline \begin{tabular}{|l}
10 \\
\end{tabular} & 4 & IA & $\mathrm{MeOH}$ & 150 \\
\hline Peak \# & Ret. Time & Area & Area $\%$ & \\
\hline \begin{tabular}{|l|}
1 \\
\end{tabular} & $10.98 \mathrm{~min}$ & 16214.0734 & \begin{tabular}{|l|l|}
4 & 50.0913
\end{tabular} & \\
\hline 2 & $14.22 \mathrm{~min}$ & 16154.9873 & \begin{tabular}{l|l}
3 & 49.9087
\end{tabular} & \\
\hline
\end{tabular}
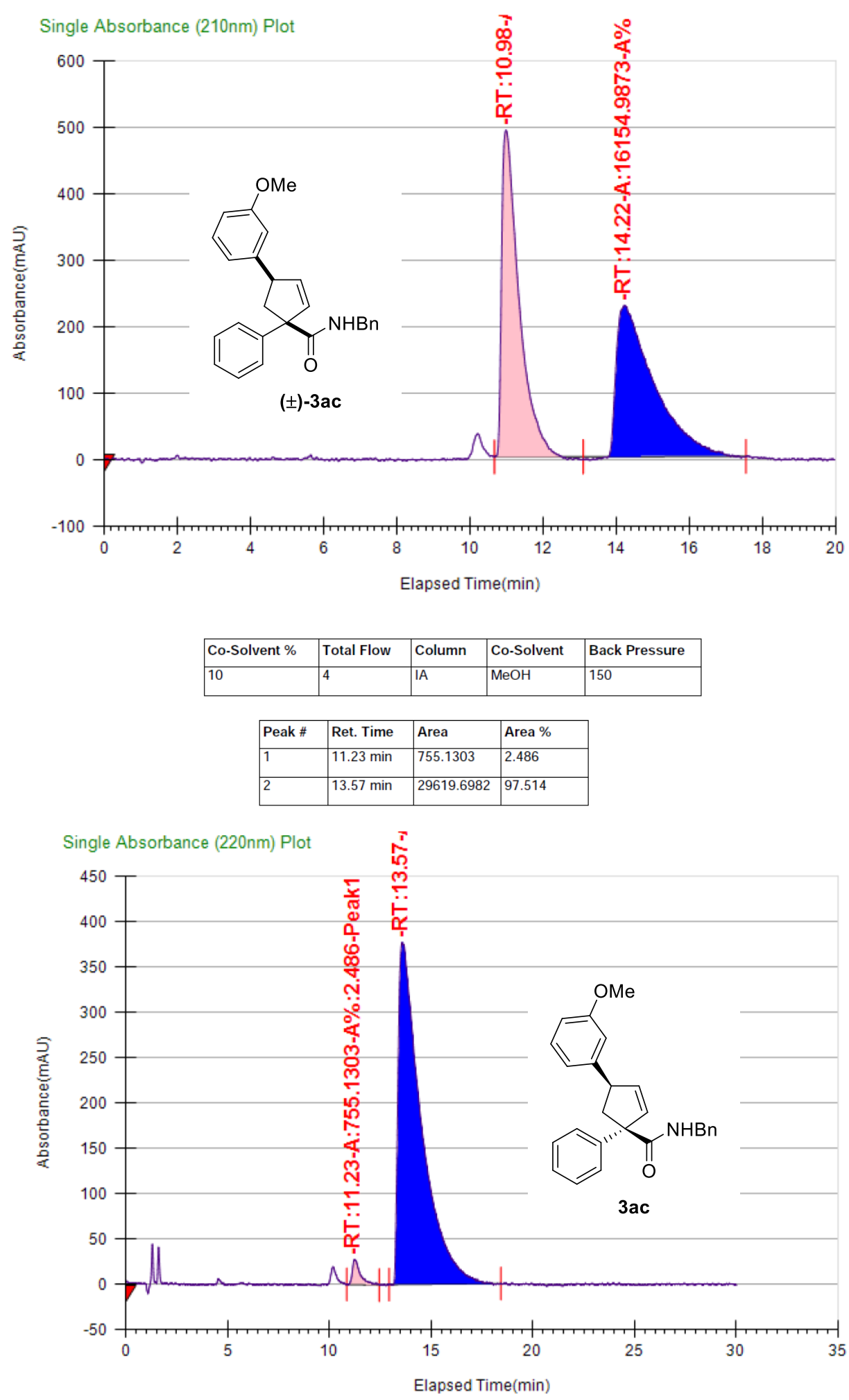


\begin{tabular}{|c|c|c|c|c|}
\hline Co-Solvent \% & Total Flow & Column & Co-Solvent & Back Pressure \\
\hline 20 & 4 & IA & $\mathrm{MeOH}$ & 150 \\
\hline Peak \# & Ret. Time & Area & Area $\%$ & \\
\hline 1 & $11.57 \mathrm{~min}$ & \begin{tabular}{|l|}
1472.8239 \\
\end{tabular} & 2.3251 & \\
\hline 2 & $12.58 \mathrm{~min}$ & 1616.9997 & 2.5527 & \\
\hline 3 & $14.59 \mathrm{~min}$ & 30041.163 & 47.4252 & \\
\hline 4 & $17.14 \mathrm{~min}$ & 30213.292 & 47.697 & \\
\hline
\end{tabular}
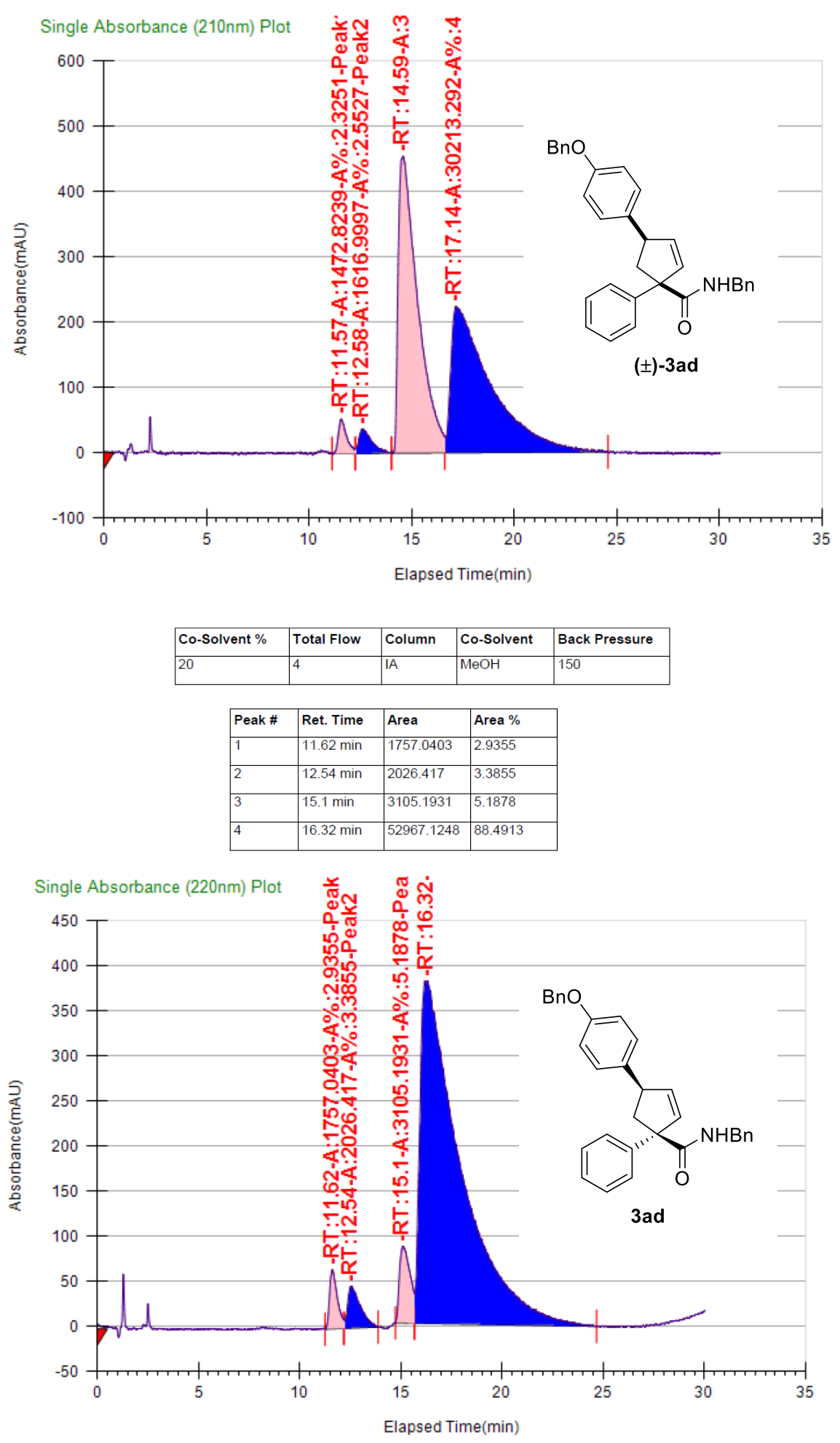


\begin{tabular}{|c|c|c|c|c|}
\hline Co-Solvent \% & Total Flow & Column & Co-Solvent & Back Pressure \\
\hline 15 & 4 & OJ-H & $\mathrm{MeOH}$ & 150 \\
\hline Peak \# & Ret. Time & Area & Area $\%$ & \\
\hline 1 & $7.68 \mathrm{~min}$ & 12327.655 & 50.1321 & \\
\hline 2 & $9.81 \mathrm{~min}$ & 12262.6734 & \begin{tabular}{|l|l|}
4 & 49.8679
\end{tabular} & \\
\hline
\end{tabular}

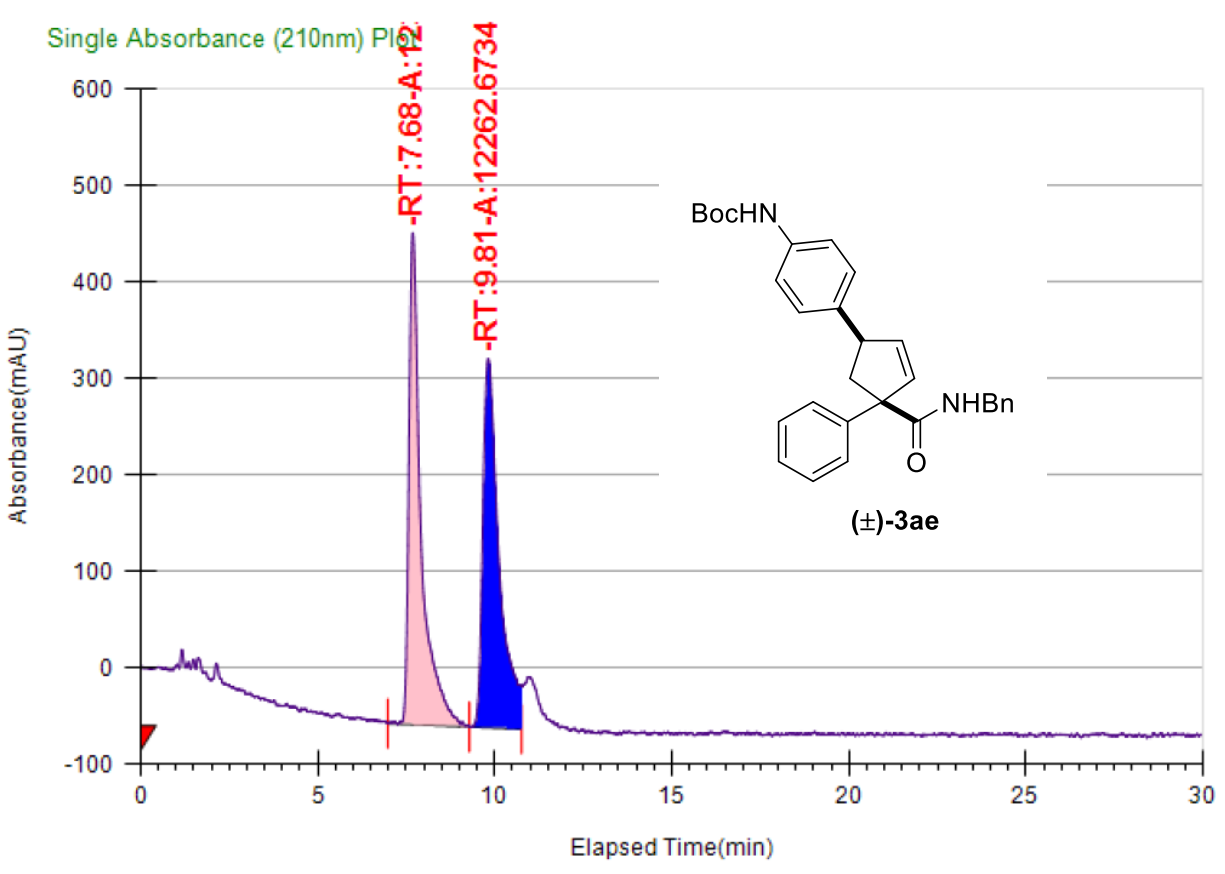

\begin{tabular}{|c|c|c|c|c|}
\hline Co-Solvent \% & Total Flow & Column & Co-Solvent & Back Pressure \\
\hline 15 & 4 & $\mathrm{OJ}-\mathrm{H}$ & $\mathrm{MeOH}$ & 150 \\
\hline Peak \# & Ret. Time & Area & Area $\%$ & \\
\hline 1 & $7.5 \mathrm{~min}$ & 487.485 & 6.128 & \\
\hline 2 & $9.49 \mathrm{~min}$ & 7467.5467 & 93.872 & \\
\hline
\end{tabular}

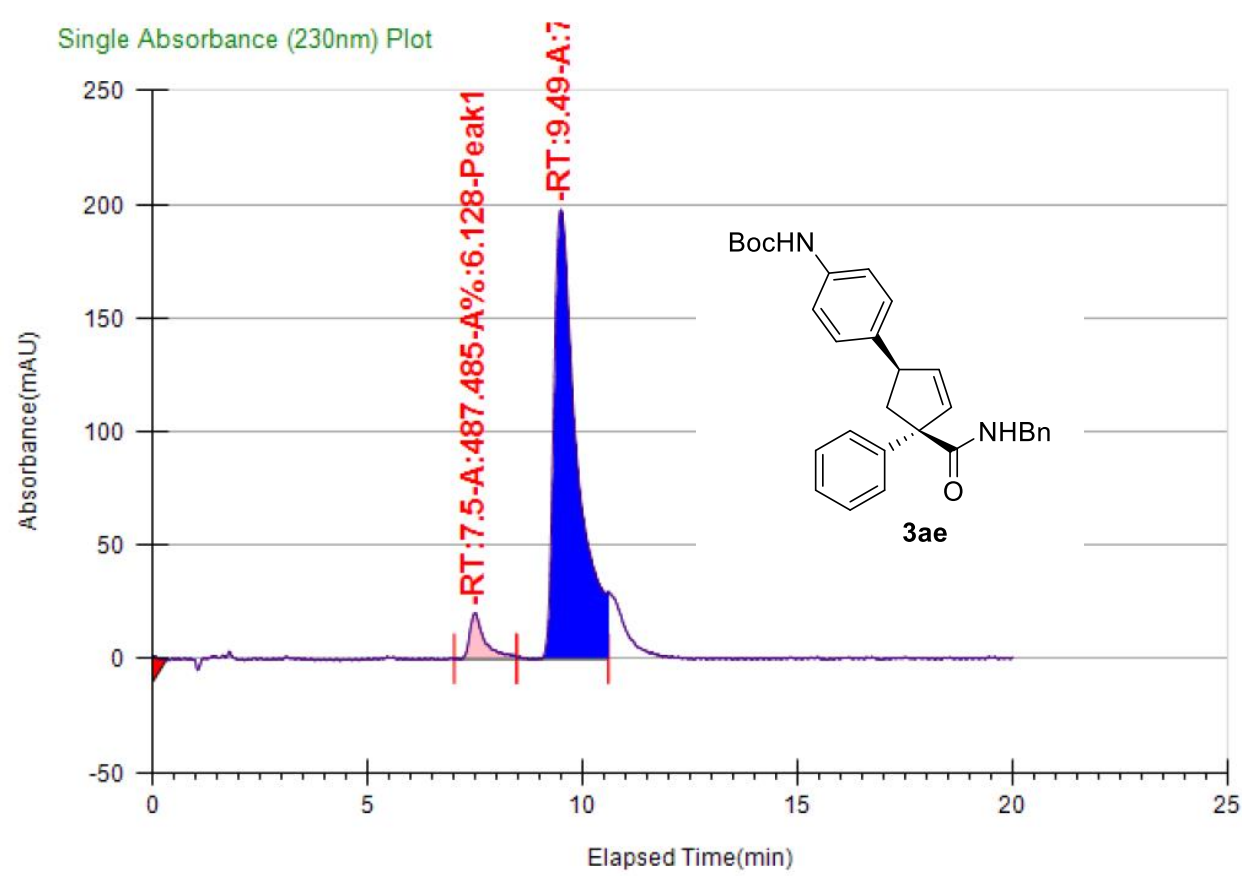




\begin{tabular}{|c|c|c|c|c|}
\hline Co-Solvent \% & Total Flow & Column & Co-Solvent & Back Pressure \\
\hline & 4 & $\mathrm{OJ}-\mathrm{H}$ & $\mathrm{MeOH}$ & 150 \\
\hline Peak \# & Ret. Time & Area & Area \% & \\
\hline 1 & $16.84 \mathrm{~min}$ & 18706.2845 & \begin{tabular}{|l|l}
5 & 49.4303
\end{tabular} & \\
\hline 2 & $20.82 \mathrm{~min}$ & 19137.4956 & \begin{tabular}{|l|l|}
50.5697 \\
\end{tabular} & \\
\hline
\end{tabular}

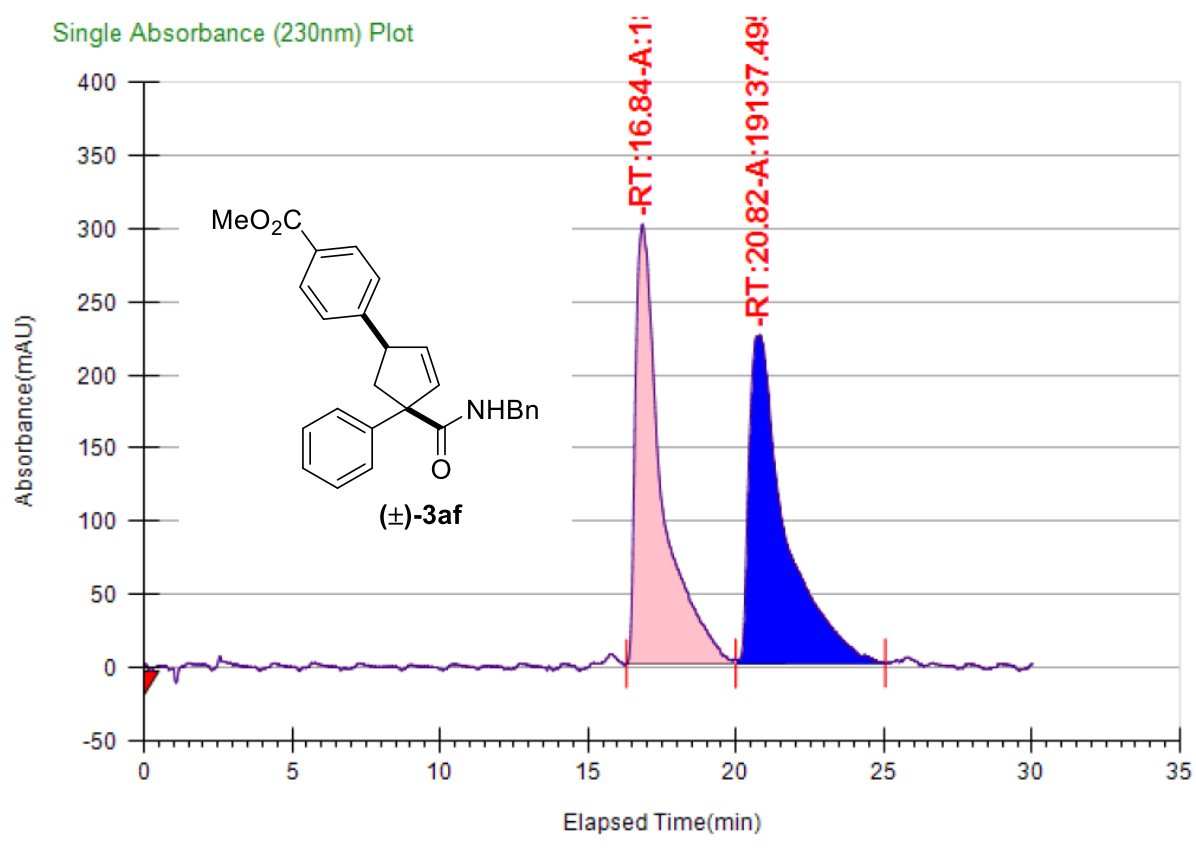

\begin{tabular}{|l|l|l|l|l|}
\hline Co-Solvent \% & Total Flow & Column & Co-Solvent & Back Pressure \\
\hline 8 & 4 & OJ-H & MeOH & 150 \\
\hline
\end{tabular}
\begin{tabular}{|l|l|l|l|}
\hline Peak \# & Ret. Time & Area & Area \% \\
\hline 1 & $16.72 \mathrm{~min}$ & 52351.8823 & 97.8977 \\
\hline 2 & 21.27 min & 1124.2504 & 2.1023 \\
\hline
\end{tabular}

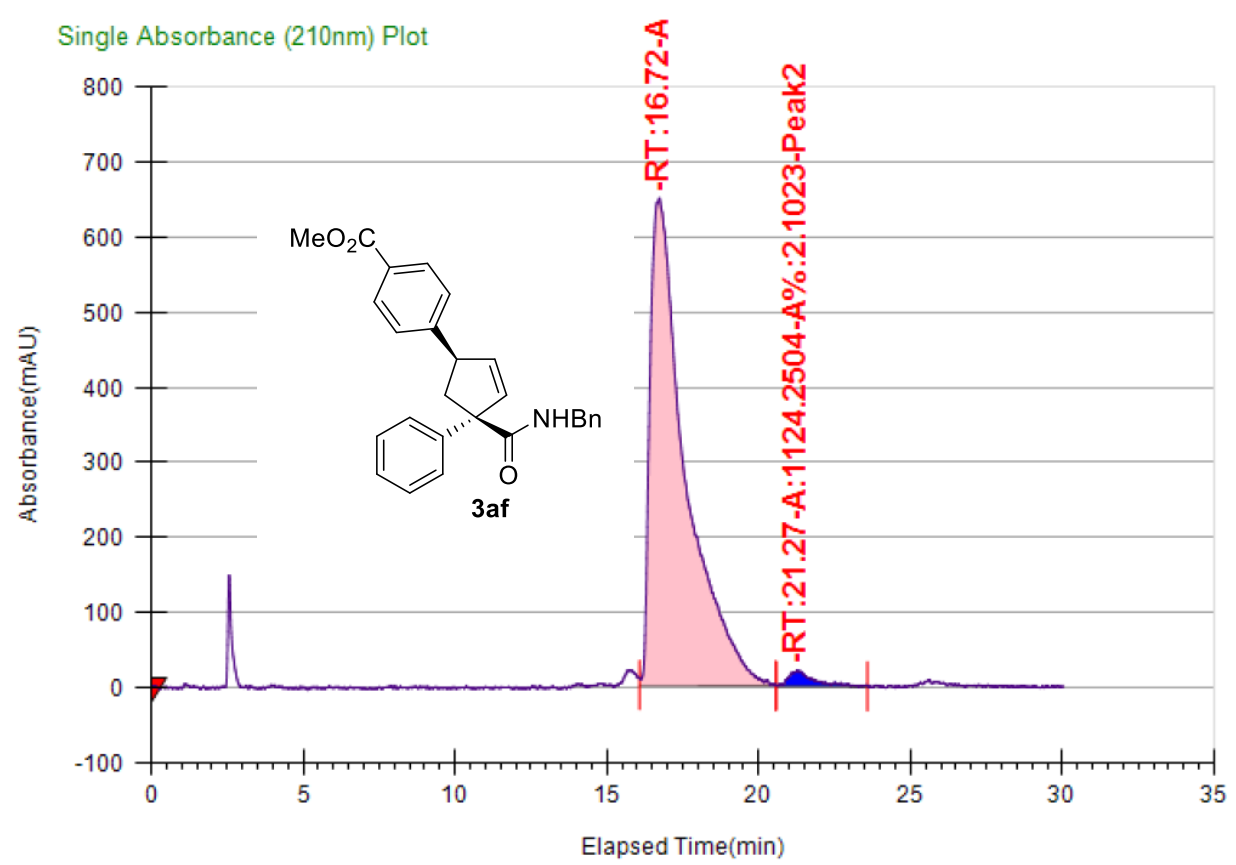




\begin{tabular}{|c|c|c|c|c|}
\hline Co-Solvent \% & Total Flow & Column & Co-Solvent & Back Pressure \\
\hline 10 & 4 & $\mathrm{OJ}-\mathrm{H}$ & $\mathrm{MeOH}$ & 150 \\
\hline Peak \# & Ret. Time & Area & Area \% & \\
\hline 1 & $8.43 \mathrm{~min}$ & 33581.627 & \begin{tabular}{l|l}
8 & 49.2479
\end{tabular} & \\
\hline 2 & $10.17 \mathrm{~min}$ & 34607.321 & \begin{tabular}{l|l|}
6 & 50.7521 \\
\end{tabular} & \\
\hline
\end{tabular}

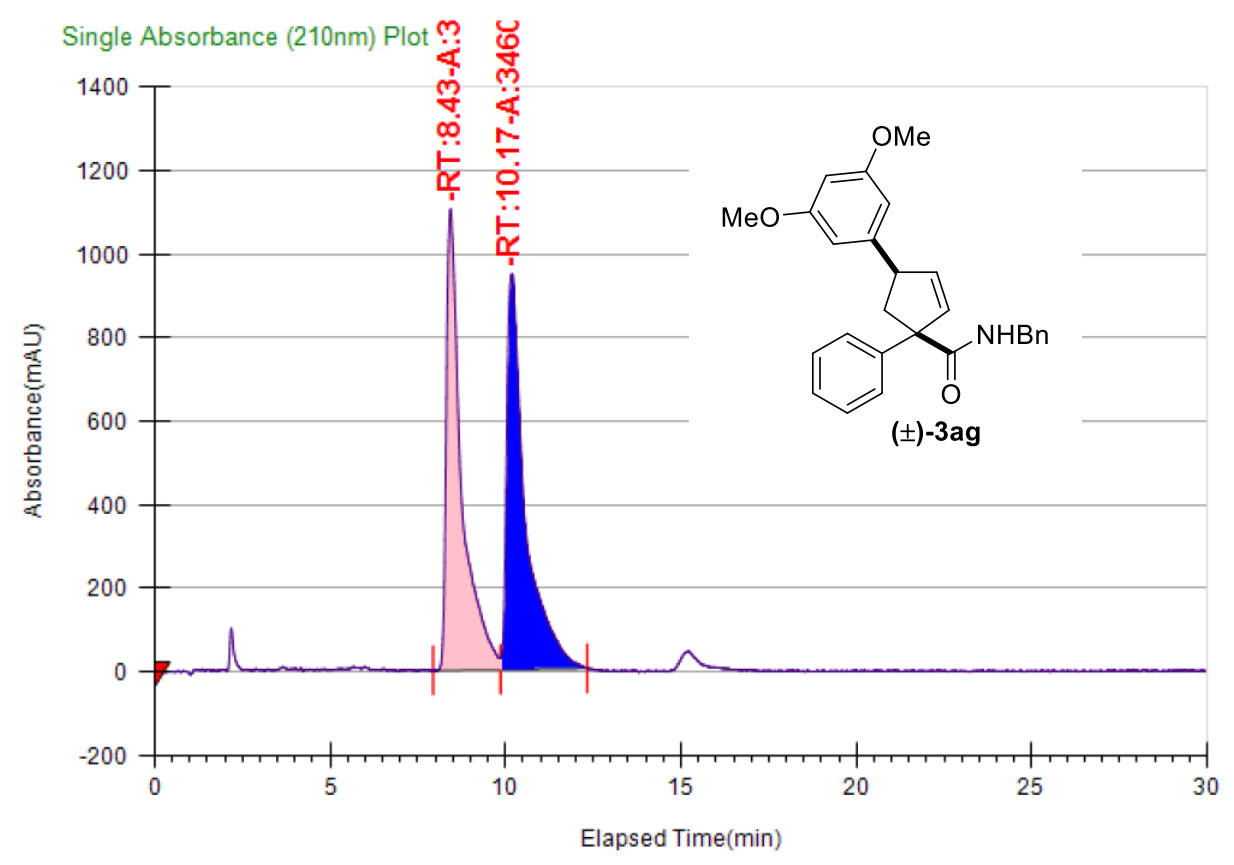

\begin{tabular}{|c|c|c|c|c|}
\hline Co-Solvent \% & Total Flow & Column & Co-Solvent & Back Pressure \\
\hline 10 & 4 & $\mathrm{OJ}-\mathrm{H}$ & $\mathrm{MeOH}$ & 150 \\
\hline Peak \# & Ret. Time & Area & Area \% & \\
\hline 1 & $8.09 \mathrm{~min}$ & 23028.1048 & \begin{tabular}{l|l|}
8 & 98.8305
\end{tabular} & \\
\hline 2 & $10.27 \mathrm{~min}$ & 272.5029 & 1.1695 & \\
\hline
\end{tabular}

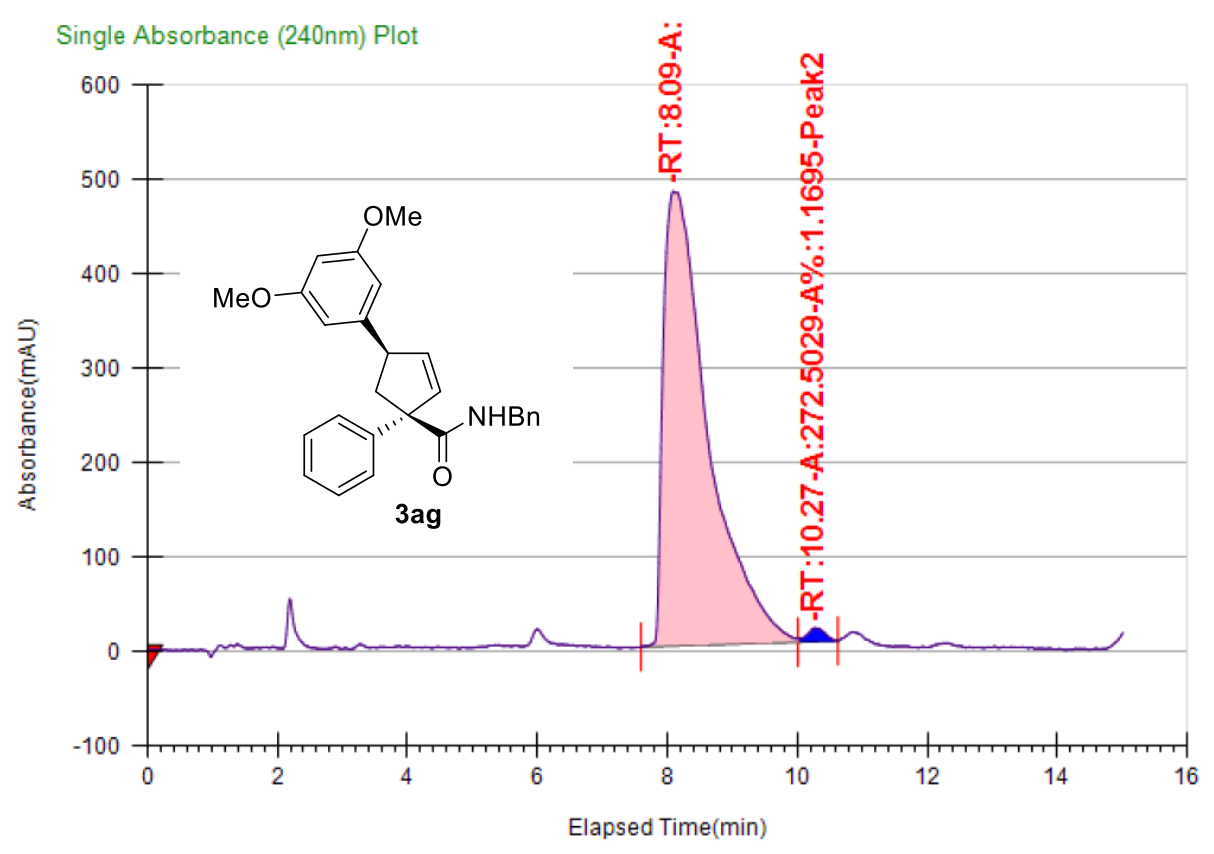




\begin{tabular}{|c|c|c|c|c|}
\hline Co-Solvent \% & Total Flow & Column & Co-Solvent & Back Pressure \\
\hline 10 & 4 & IA & $\mathrm{MeOH}$ & 150 \\
\hline Peak \# & Ret. Time & Area & Area $\%$ & \\
\hline 1 & $8.89 \mathrm{~min}$ & 10176.7868 & \begin{tabular}{|l|l}
8 & 50.7503
\end{tabular} & \\
\hline 2 & $10.7 \mathrm{~min}$ & 9875.8815 & 49.2497 & \\
\hline
\end{tabular}

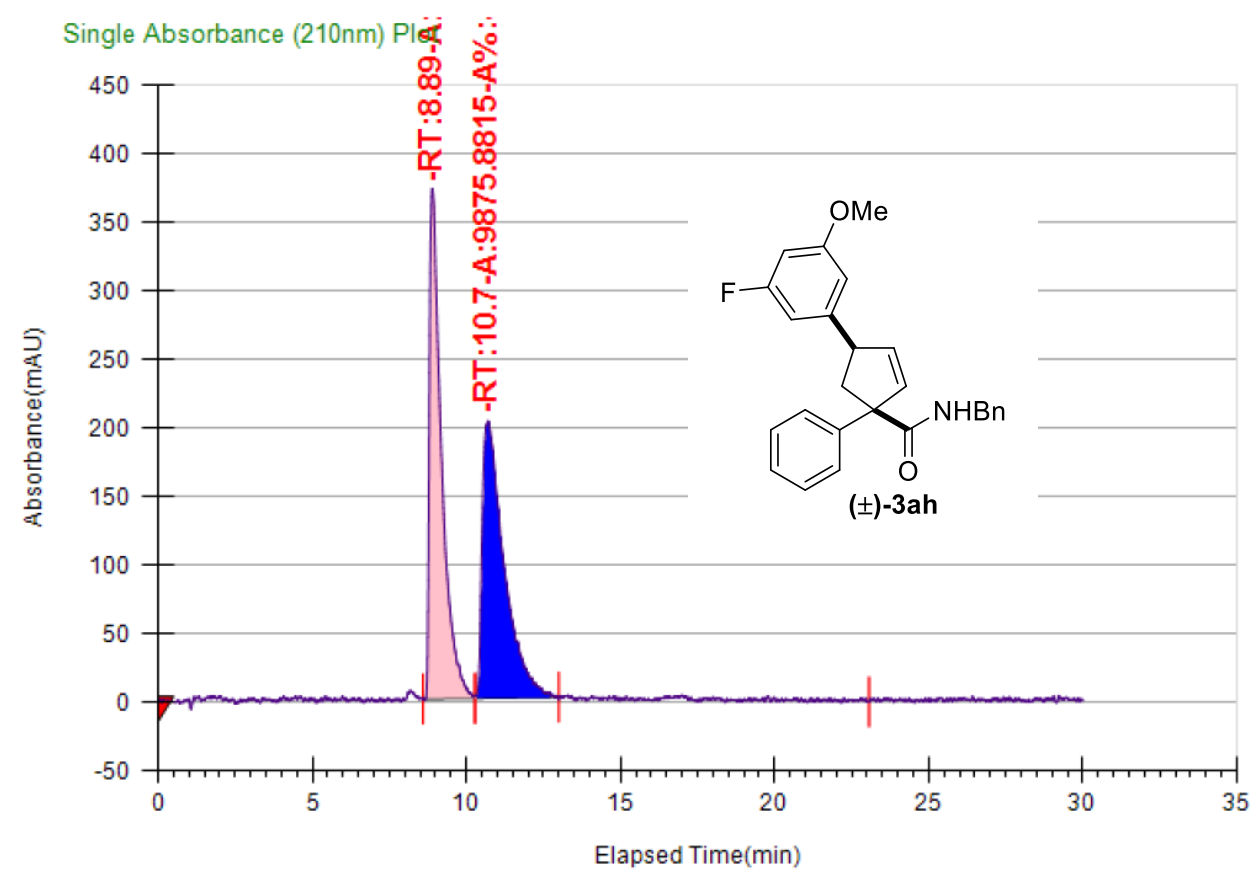

\begin{tabular}{|l|l|l|l|l|}
\hline Co-Solvent \% & Total Flow & Column & Co-Solvent & Back Pressure \\
\hline 10 & 4 & IA & MeOH & 150 \\
\hline
\end{tabular}
\begin{tabular}{ll|l|l|}
\hline Peak \# & Ret. Time & Area & Area \% \\
\hline 1 & 8.88 min & 2414.599 & 2.4359 \\
\hline 2 & 9.64 min & 96711.5841 & 97.5641 \\
\hline
\end{tabular}

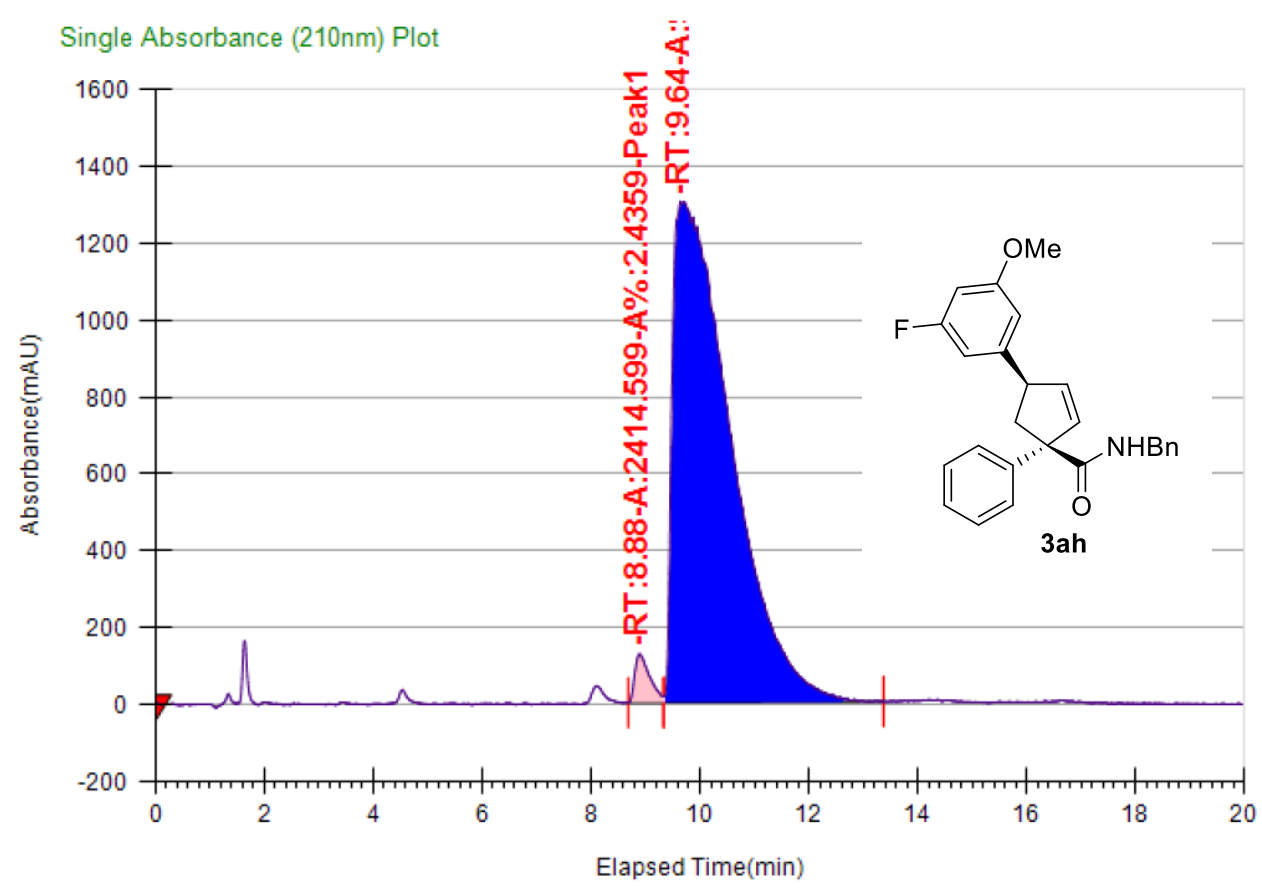




\begin{tabular}{|l|l|l|l|l|}
\hline Co-Solvent $\%$ & Total Flow & Column & Co-Solvent & Back Pressure \\
\hline 10 & 4 & IA & MeOH & 150 \\
\hline
\end{tabular}
\begin{tabular}{|l|l|l|l|}
\hline Peak \# & Ret. Time & Area & Area \% \\
\hline 1 & 11.11 min & 9917.7664 & 50.5232 \\
\cline { 1 - 4 } & 12.4 min & 9712.3572 & 49.4768 \\
\hline
\end{tabular}
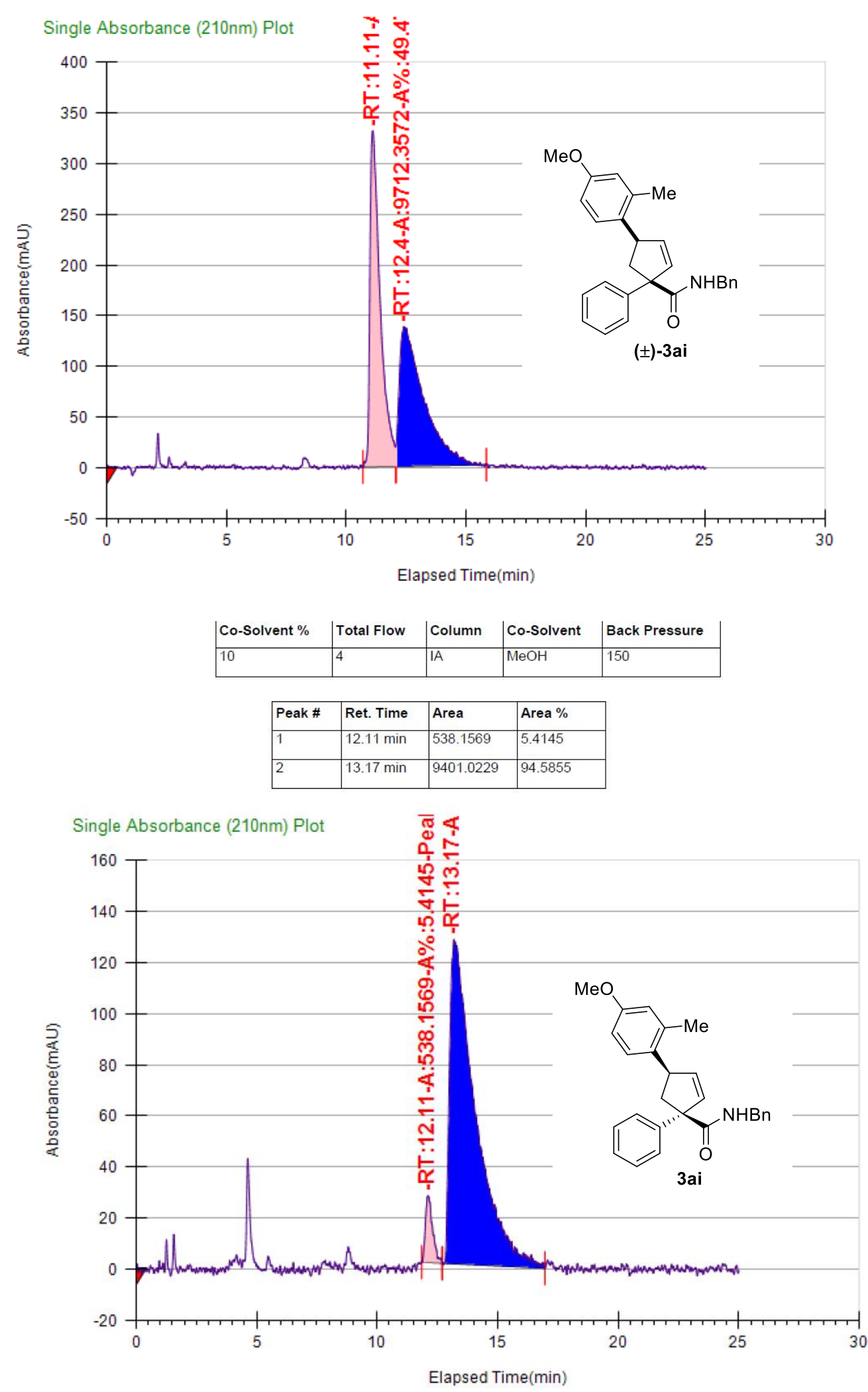


\begin{tabular}{|l|l|l|l|l|}
\hline Co-Solvent $\%$ & Total Flow & Column & Co-Solvent & Back Pressure \\
\hline 20 & 4 & IA & MeOH & 150 \\
\hline
\end{tabular}
\begin{tabular}{|l|l|l|l|}
\hline Peak \# & Ret. Time & Area & Area \% \\
\hline 1 & 9.48 min & 2022.1402 & 3.4091 \\
\hline 2 & 10.02 min & 27672.0601 & 46.6522 \\
\hline 3 & 11.8 min & 2291.2801 & 3.8629 \\
\hline 4 & 12.83 min & 27330.1376 & 46.0758 \\
\hline
\end{tabular}
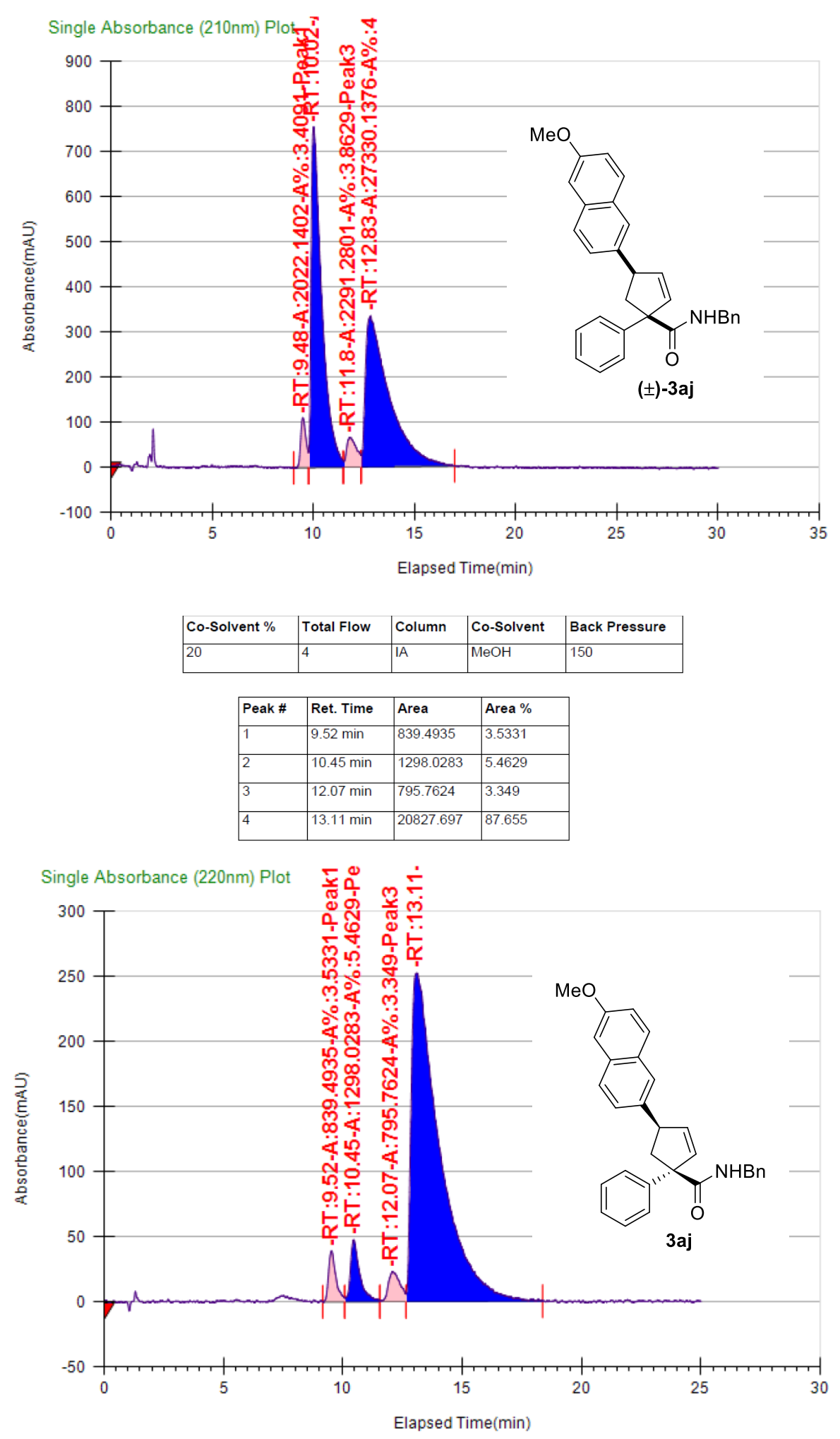


\begin{tabular}{|c|c|c|c|c|}
\hline Co-Solvent \% & Total Flow & Column & Co-Solvent & Back Pressure \\
\hline 10 & 4 & $\mathrm{OJ}-\mathrm{H}$ & $\mathrm{MeOH}$ & 150 \\
\hline Peak \# & Ret. Time & Area & Area \% & \\
\hline 1 & $11.21 \mathrm{~min}$ & 15686.0285 & \begin{tabular}{l|l}
5 & 48.5134
\end{tabular} & \\
\hline 2 & $12.87 \mathrm{~min}$ & 16647.365 & 51.4866 & \\
\hline
\end{tabular}

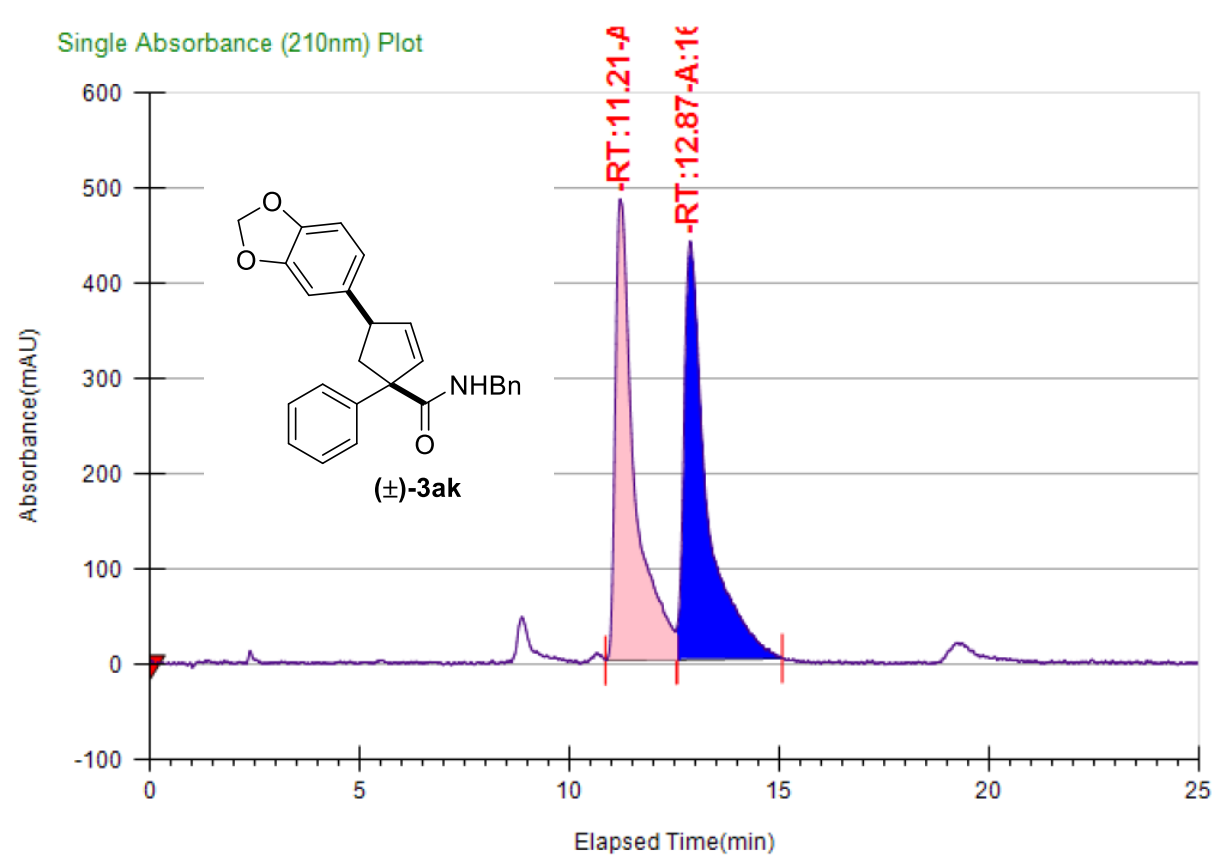

\begin{tabular}{|c|c|c|c|c|}
\hline Co-Solvent \% & Total Flow & Column & Co-Solvent & Back Pressure \\
\hline 10 & 4 & $\mathrm{OJ}-\mathrm{H}$ & $\mathrm{MeOH}$ & 150 \\
\hline Peak \# & Ret. Time & Area & Area $\%$ & \\
\hline 1 & $10.83 \mathrm{~min}$ & 46491.7122 & \begin{tabular}{|l|l|}
2 & 94.1685
\end{tabular} & \\
\hline 2 & $12.8 \mathrm{~min}$ & 2879.0593 & 5.8315 & \\
\hline
\end{tabular}

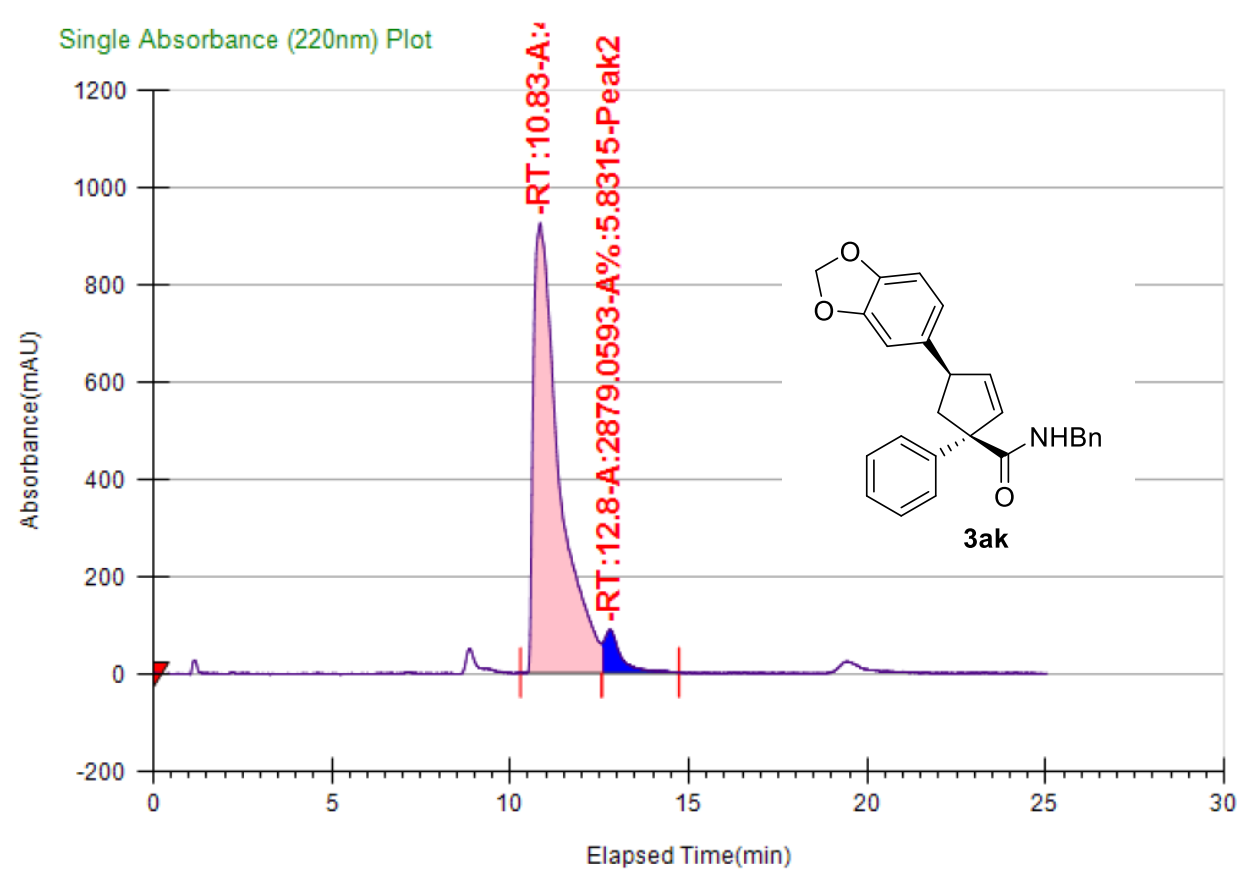




\begin{tabular}{|c|c|c|c|c|}
\hline Co-Solvent \% & Total Flow & Column & Co-Solvent & Back Pressure \\
\hline 10 & 4 & IA & $\mathrm{MeOH}$ & 150 \\
\hline Peak \# & Ret. Time & Area & Area \% & \\
\hline 1 & $16.01 \mathrm{~min}$ & 37408.4005 & \begin{tabular}{|l|l|}
5 & 48.4629 \\
\end{tabular} & \\
\hline 2 & $18.55 \mathrm{~min}$ & 39781.3715 & \begin{tabular}{l|l|}
5 & 51.5371
\end{tabular} & \\
\hline
\end{tabular}

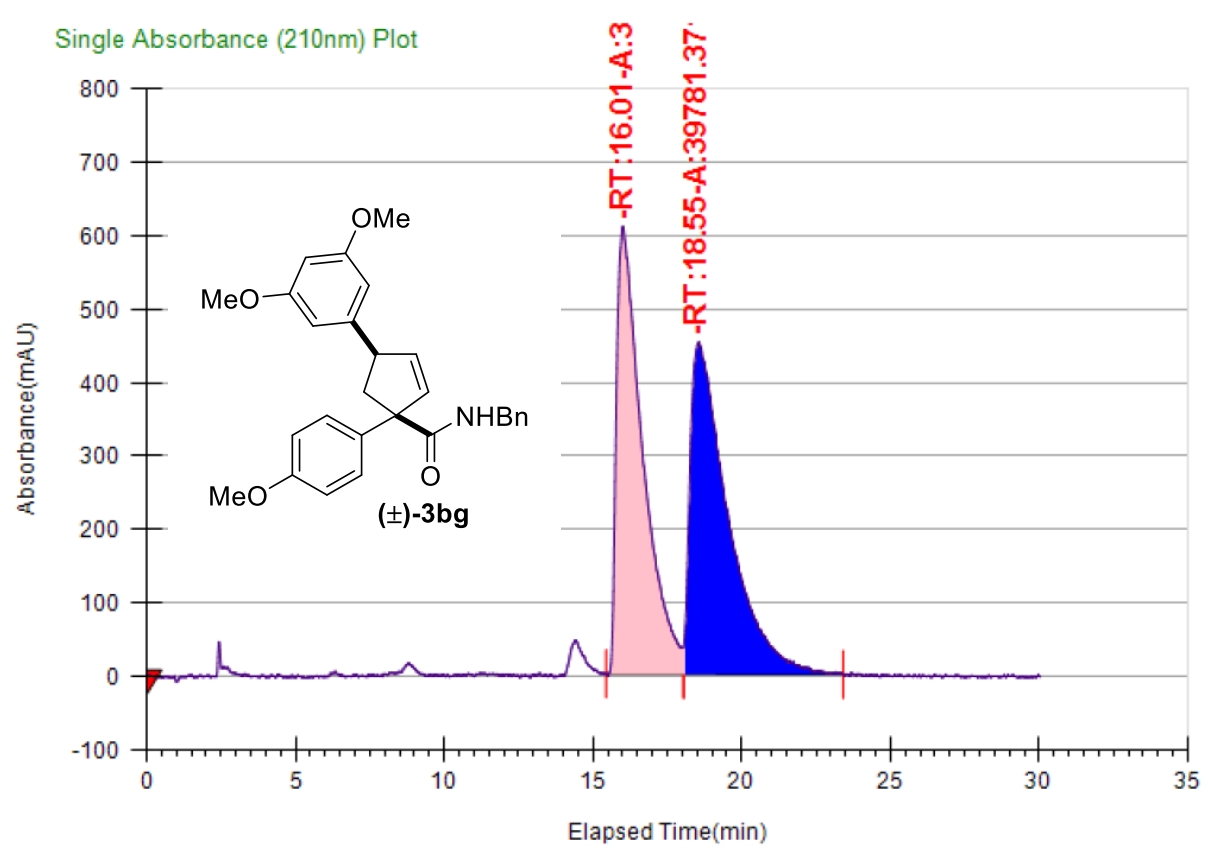

\begin{tabular}{|c|c|c|c|c|}
\hline Co-Solvent \% & Total Flow & Column & Co-Solvent & Back Pressure \\
\hline 10 & 4 & IA & $\mathrm{MeOH}$ & 150 \\
\hline Peak \# & Ret. Time & Area & Area \% & \\
\hline 1 & $16.01 \mathrm{~min}$ & 1850.5515 & 1.7375 & \\
\hline 2 & $17.06 \mathrm{~min}$ & 104658.4055 & \begin{tabular}{l|l}
55 & 98.2625
\end{tabular} & \\
\hline
\end{tabular}

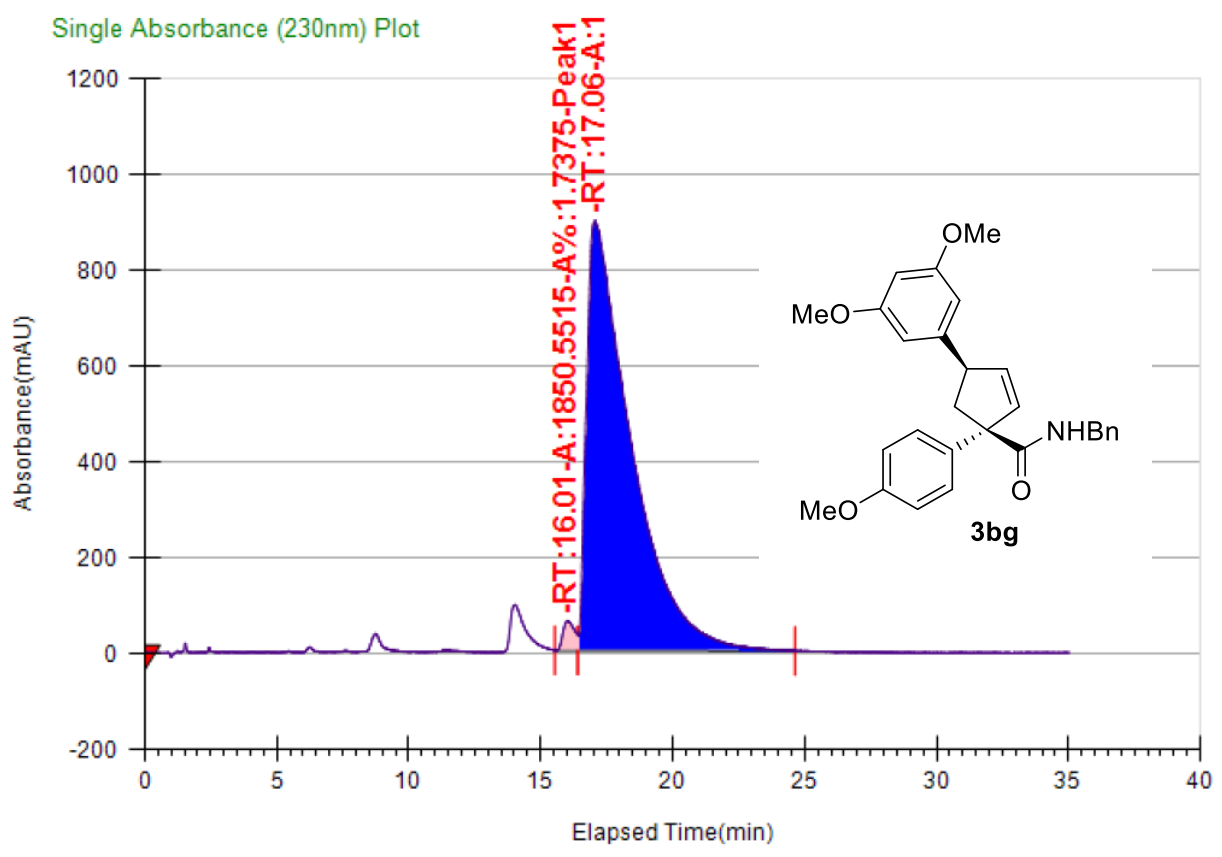




\begin{tabular}{|c|c|c|c|c|}
\hline Co-Solvent \% & Total Flow & Column & Co-Solvent & Back Pressure \\
\hline 10 & 4 & IA & $\mathrm{MeOH}$ & 150 \\
\hline Peak \# & Ret. Time & Area & Area $\%$ & \\
\hline 1 & $9.42 \mathrm{~min}$ & 28453.2465 & \begin{tabular}{|l|l|}
5 & 50.2142 \\
\end{tabular} & \\
\hline 2 & $11.03 \mathrm{~min}$ & 28210.4501 & \begin{tabular}{|l|l|} 
& 49.7858 \\
\end{tabular} & \\
\hline
\end{tabular}

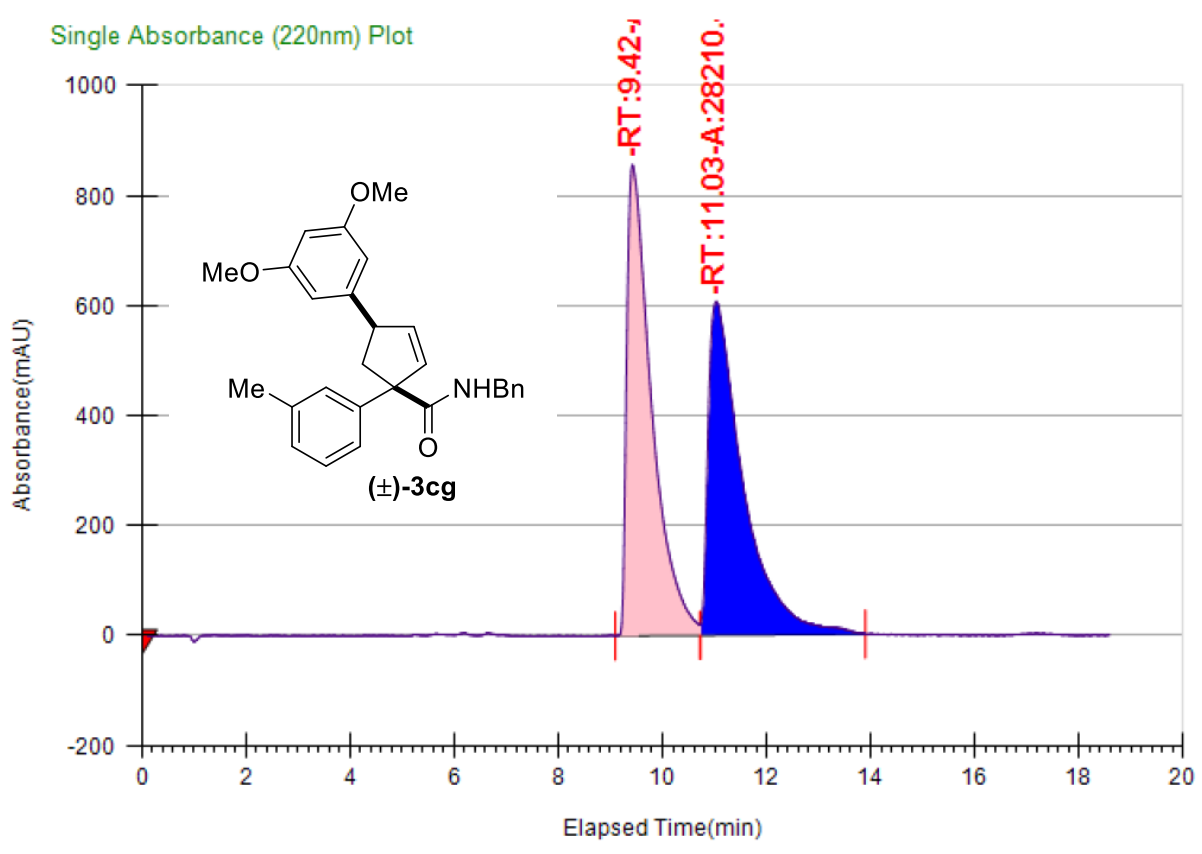

\begin{tabular}{|c|c|c|c|c|}
\hline Co-Solvent \% & Total Flow & Column & Co-Solvent & Back Pressure \\
\hline 10 & 4 & IA & $\mathrm{MeOH}$ & 150 \\
\hline Peak \# & Ret. Time & Area & Area \% & \\
\hline 1 & $9.17 \mathrm{~min}$ & 3321.3446 & 5.4742 & \\
\hline 2 & $10.06 \mathrm{~min}$ & 57351.3253 & \begin{tabular}{|l|l|}
3 & 94.5258 \\
\end{tabular} & \\
\hline
\end{tabular}

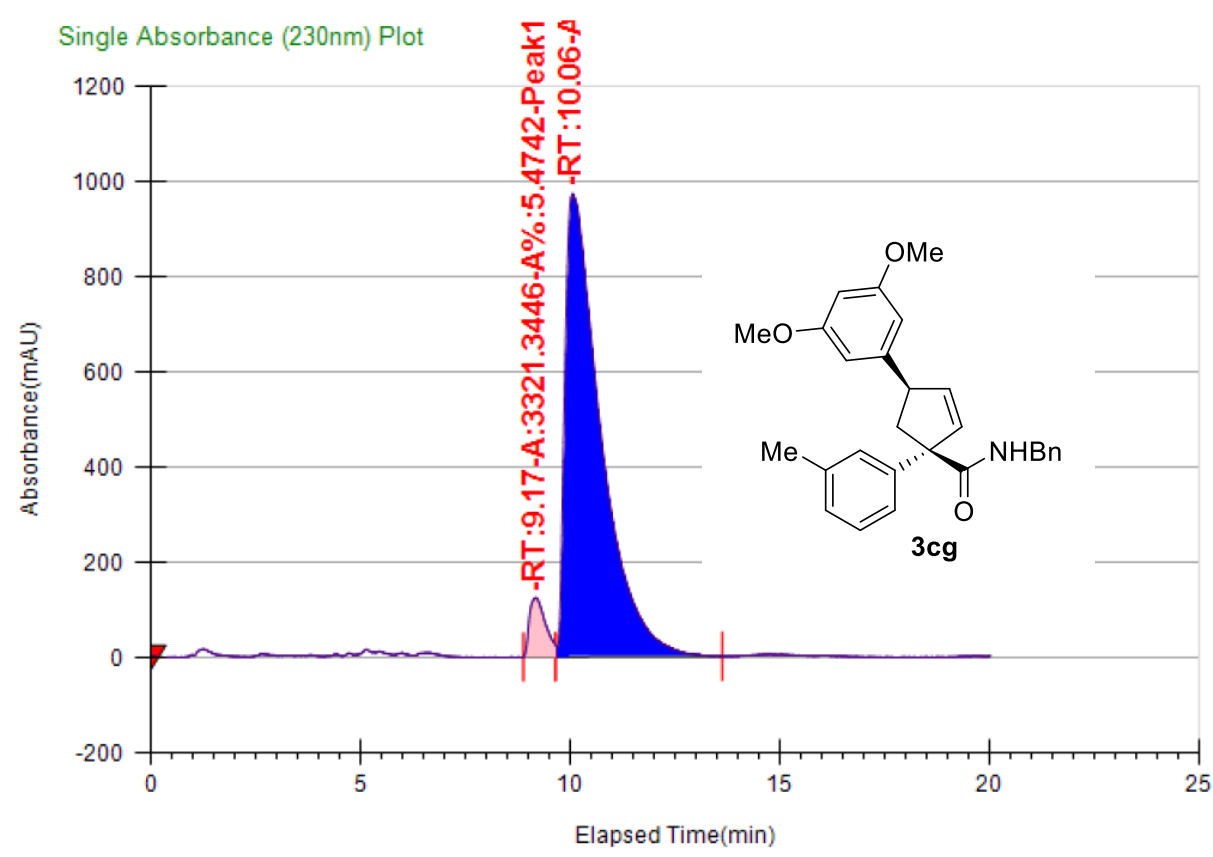




\begin{tabular}{|c|c|c|c|c|}
\hline Co-Solvent \% & Total Flow & Column & Co-Solvent & Back Pressure \\
\hline 10 & 4 & IC & $\mathrm{MeOH}$ & 150 \\
\hline Peak \# & Ret. Time & Area & Area $\%$ & \\
\hline \begin{tabular}{|l}
1 \\
1
\end{tabular} & $11.37 \mathrm{~min}$ & 39279.9258 & \begin{tabular}{l|l}
8 & 49.074
\end{tabular} & \\
\hline 2 & $14.17 \mathrm{~min}$ & 40762.3623 & \begin{tabular}{l|l}
3 & 50.926
\end{tabular} & \\
\hline
\end{tabular}

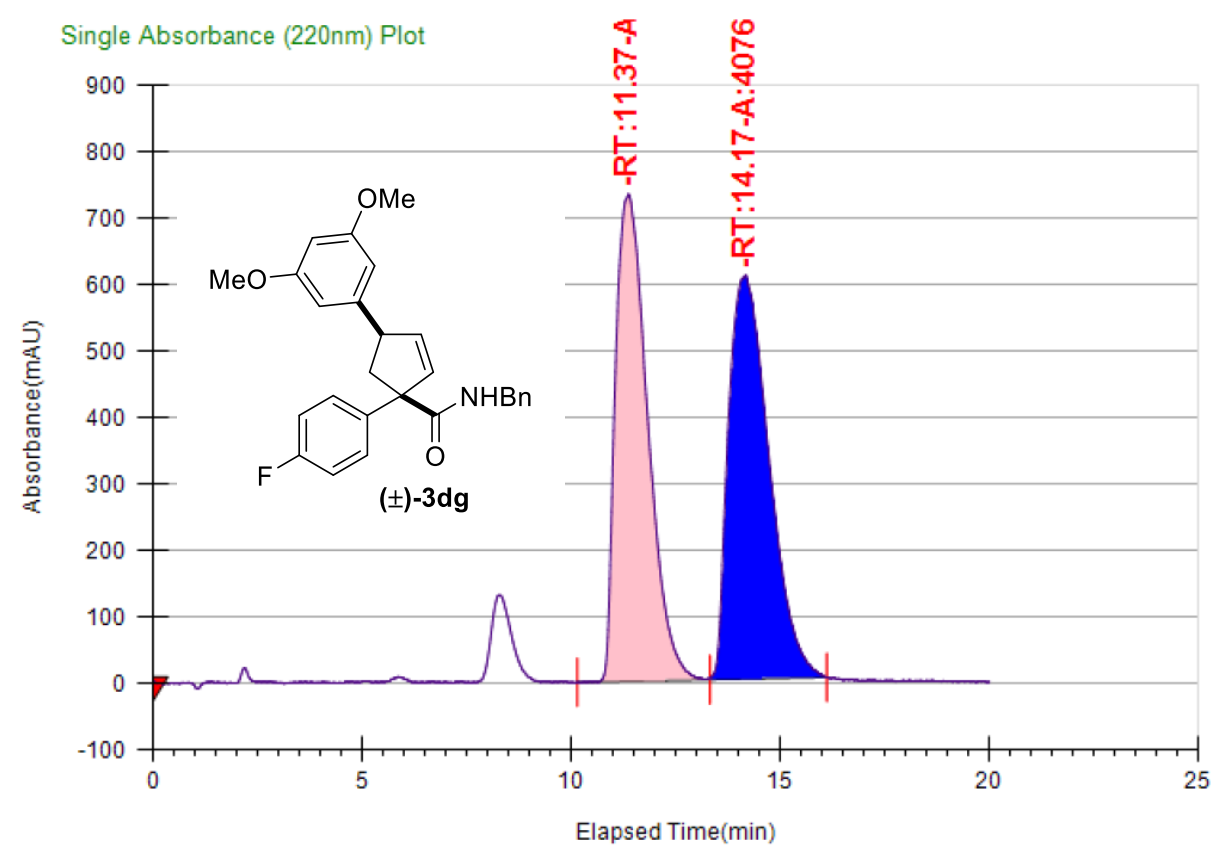

\begin{tabular}{|c|c|c|c|c|}
\hline Co-Solvent \% & Total Flow & Column & Co-Solvent & Back Pressure \\
\hline 10 & 4 & IC & $\mathrm{MeOH}$ & 150 \\
\hline Peak \# & Ret. Time & Area & Area $\%$ & \\
\hline 1 & $10.81 \mathrm{~min}$ & 24237.2324 & \begin{tabular}{l|l}
4 & 95.5257
\end{tabular} & \\
\hline 2 & $14.13 \mathrm{~min}$ & 1135.2521 & 4.4743 & \\
\hline
\end{tabular}

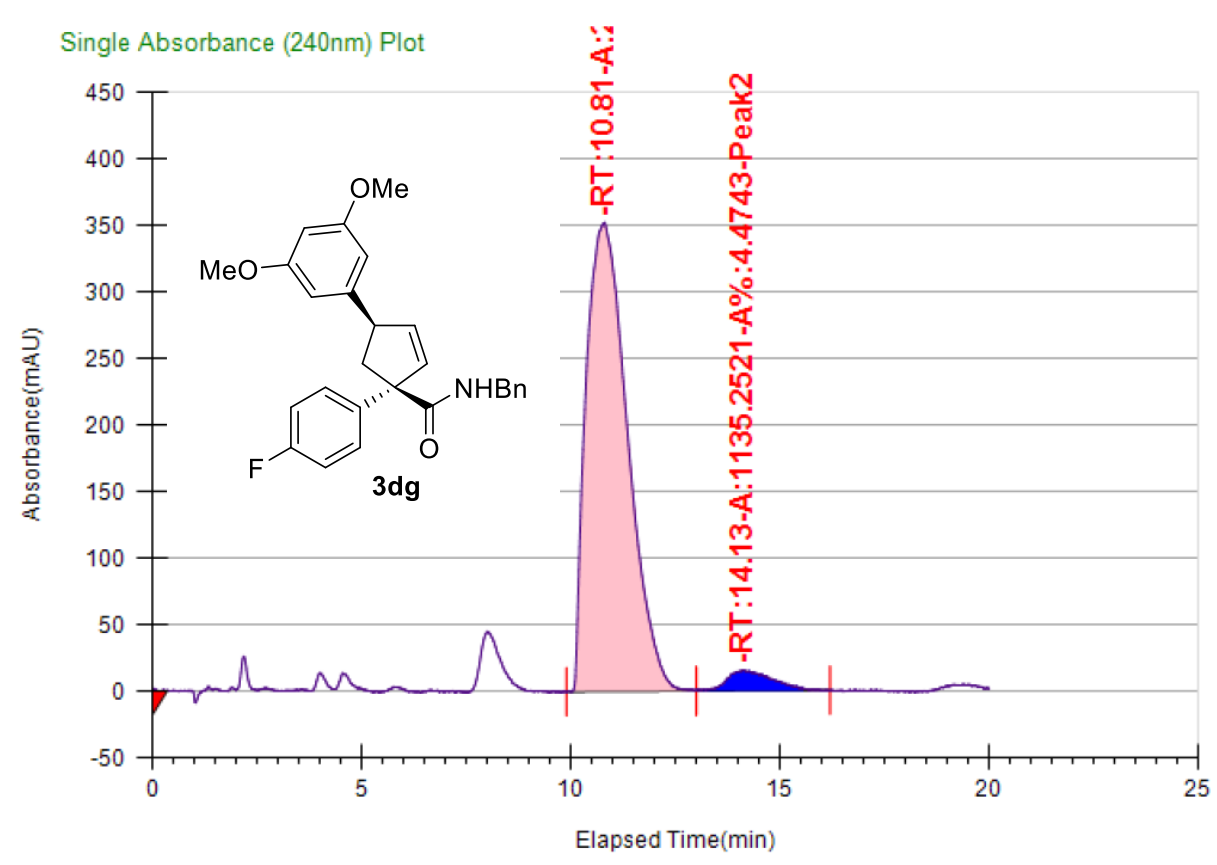




\begin{tabular}{|l|l|l|l|l|}
\hline Co-Solvent \% & Total Flow & Column & Co-Solvent & Back Pressure \\
\hline 15 & 4 & IA & $\mathrm{MeOH}$ & 150 \\
\hline
\end{tabular}

\begin{tabular}{|l|l|l|l|}
\hline Peak \# & Ret. Time & Area & Area \% \\
\hline 1 & $7.67 \mathrm{~min}$ & 40726.9199 & 49.632 \\
\hline 2 & $10.47 \mathrm{~min}$ & 41330.8188 & 50.368 \\
\hline
\end{tabular}

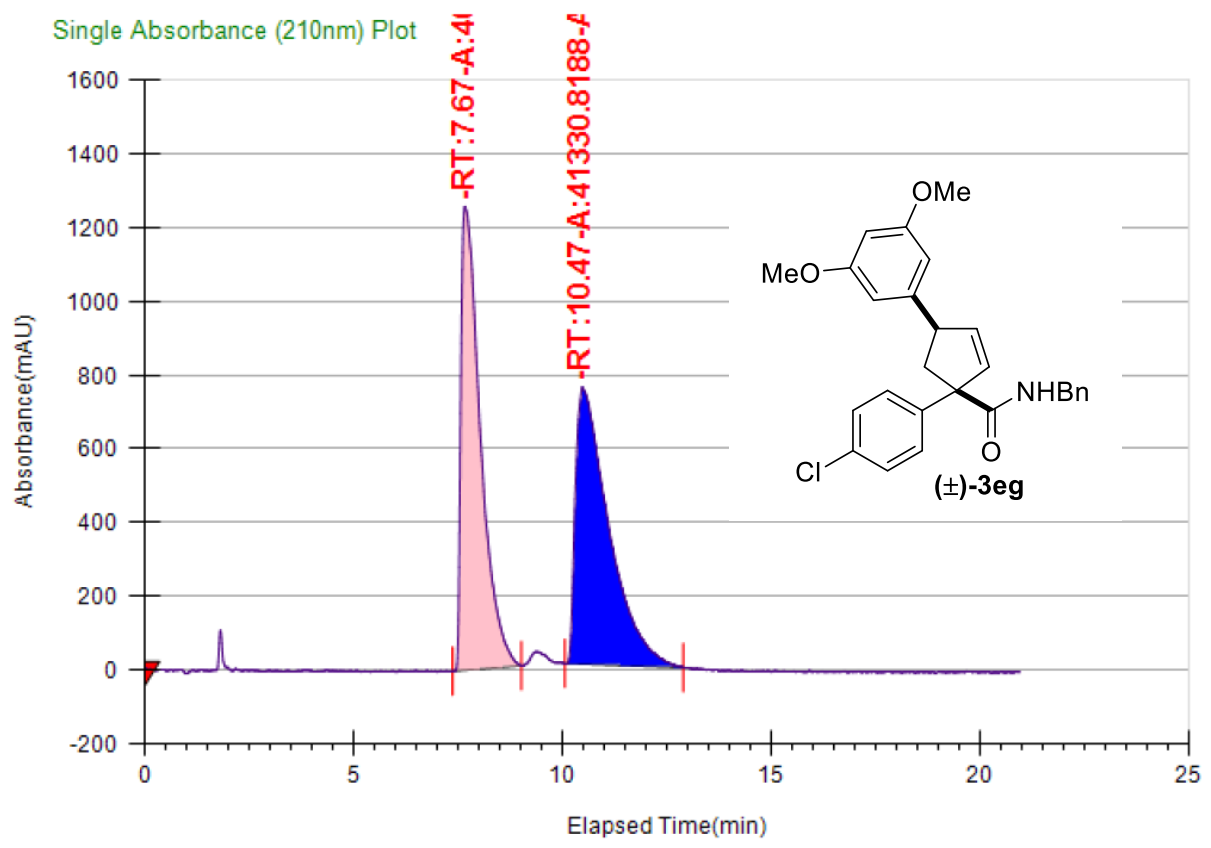

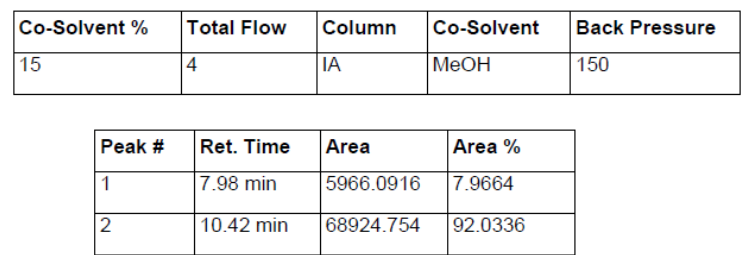

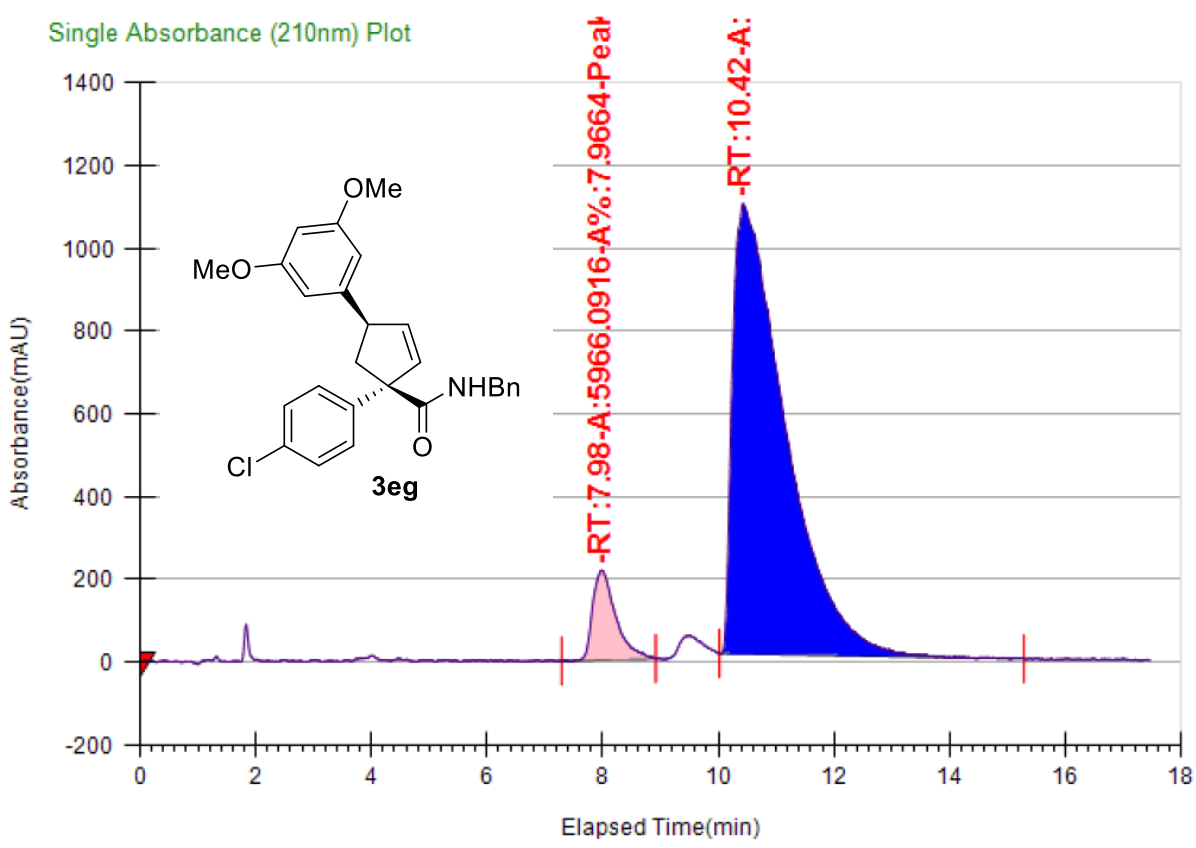




\begin{tabular}{|c|c|c|c|c|}
\hline Co-Solvent \% & Total Flow & Column & Co-Solvent & Back Pressure \\
\hline 10 & 4 & AS-H & $\mathrm{MeOH}$ & 150 \\
\hline Peak \# & Ret. Time & Area & Area \% & \\
\hline 1 & $5.38 \mathrm{~min}$ & 34912.708 & \begin{tabular}{l|l}
4 & 49.8531
\end{tabular} & \\
\hline 2 & $7.49 \mathrm{~min}$ & 35118.4453 & \begin{tabular}{|l|l|}
3 & 50.1469
\end{tabular} & \\
\hline
\end{tabular}
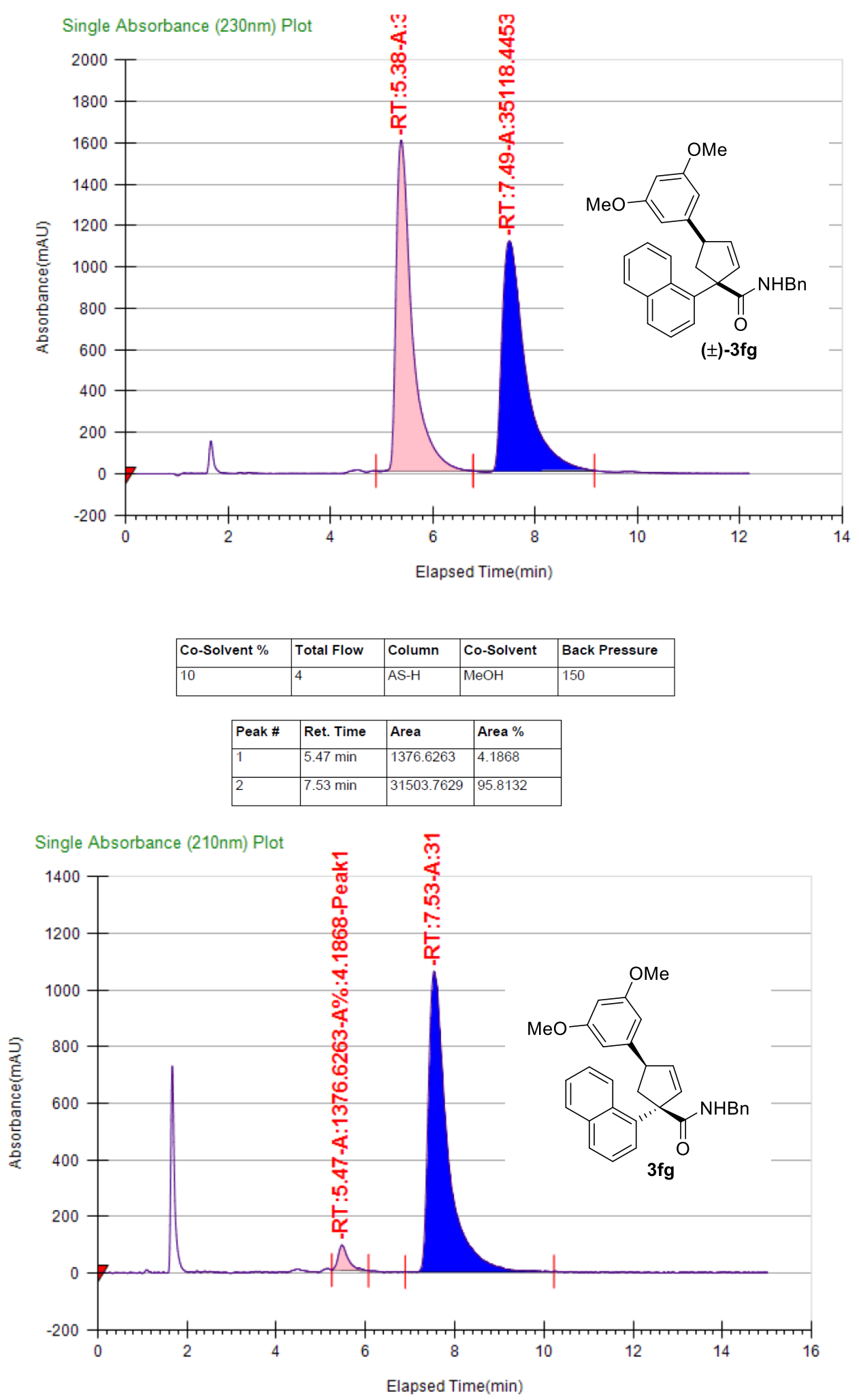


\begin{tabular}{|c|c|c|c|c|}
\hline Co-Solvent \% & Total Flow & Column & Co-Solvent & Back Pressure \\
\hline 10 & 4 & AS-H & $\mathrm{MeOH}$ & 150 \\
\hline Peak \# & Ret. Time & Area & Area $\%$ & \\
\hline \begin{tabular}{|l|}
1 \\
1
\end{tabular} & $4.95 \mathrm{~min}$ & 5853.2178 & 50.8844 & \\
\hline 2 & $6.08 \mathrm{~min}$ & 5649.755 & 49.1156 & \\
\hline
\end{tabular}

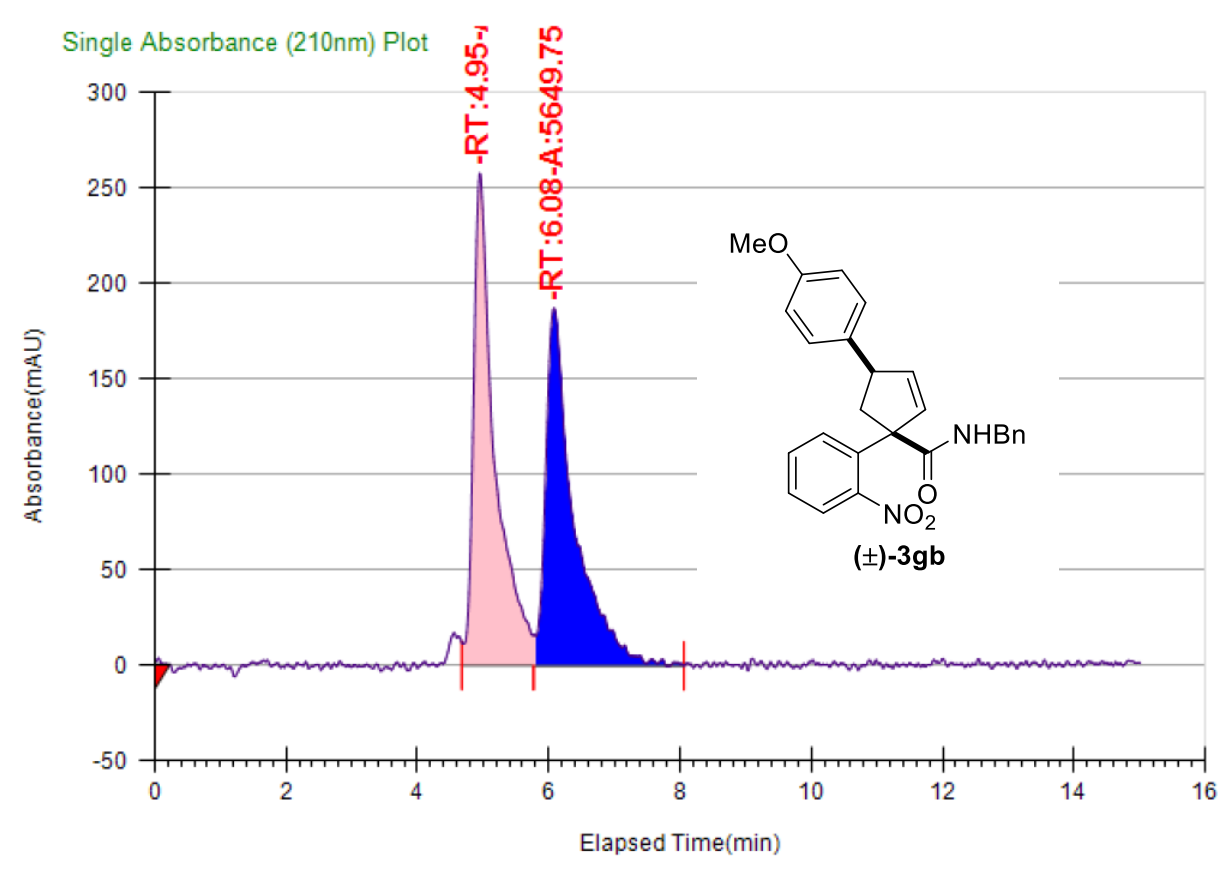

\begin{tabular}{|c|c|c|c|c|}
\hline Co-Solvent \% & Total Flow & Column & Co-Solvent & Back Pressure \\
\hline 10 & 4 & AS-H & $\mathrm{MeOH}$ & 150 \\
\hline Peak \# & Ret. Time & Area & Area $\%$ & \\
\hline 1 & $4.81 \mathrm{~min}$ & 8145.1922 & 15.9067 & \\
\hline 2 & $5.8 \mathrm{~min}$ & 43060.8258 & \begin{tabular}{l|l}
8 & 84.0933
\end{tabular} & \\
\hline
\end{tabular}

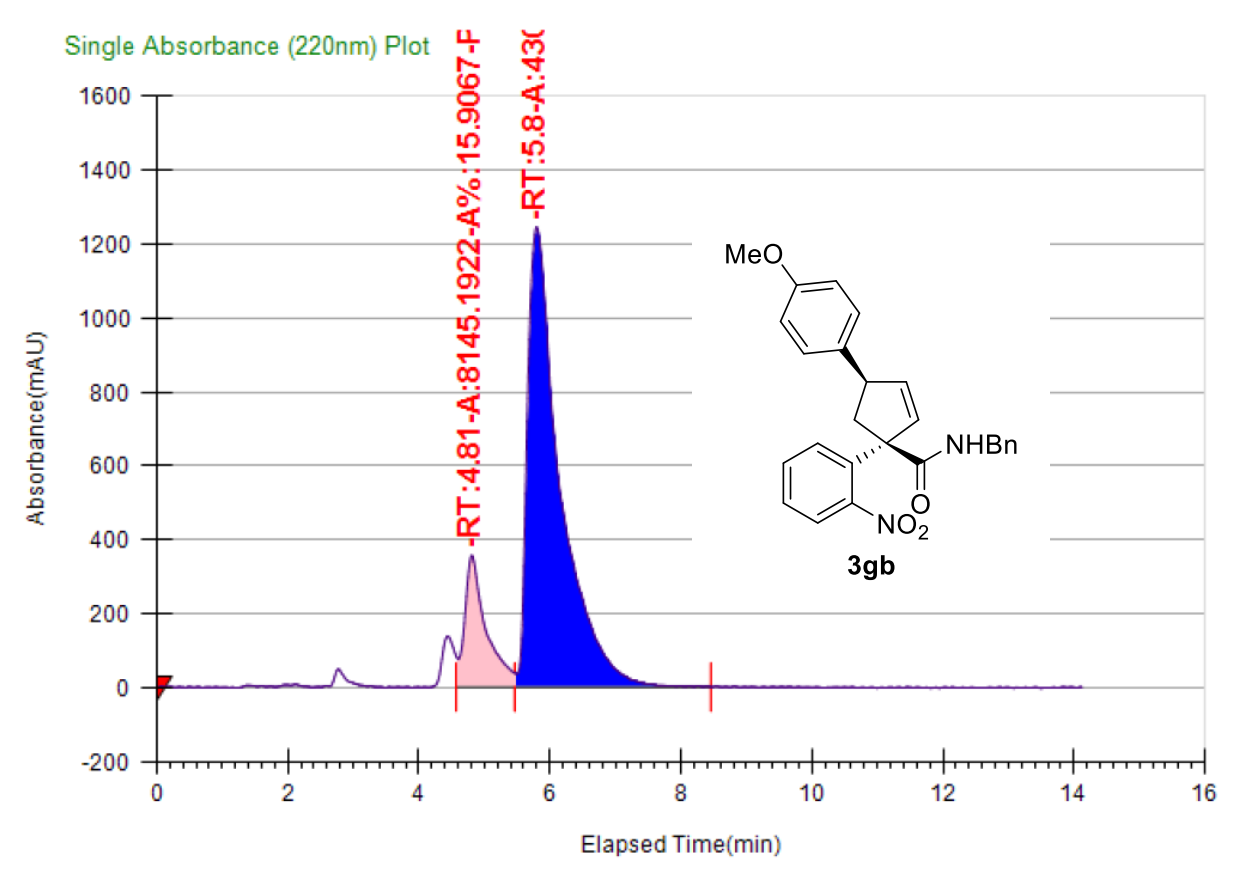




\begin{tabular}{|c|c|c|c|c|}
\hline Co-Solvent \% & Total Flow & Column & Co-Solvent & Back Pressure \\
\hline 10 & 4 & IC & $\mathrm{MeOH}$ & 150 \\
\hline Peak \# & Ret. Time & Area & Area \% & \\
\hline 1 & $13.77 \mathrm{~min}$ & 24103.784 & 50.1174 & \\
\hline 2 & $15.67 \mathrm{~min}$ & 23990.8902 & \begin{tabular}{l|l|}
2 & 49.8826 \\
\end{tabular} & \\
\hline
\end{tabular}

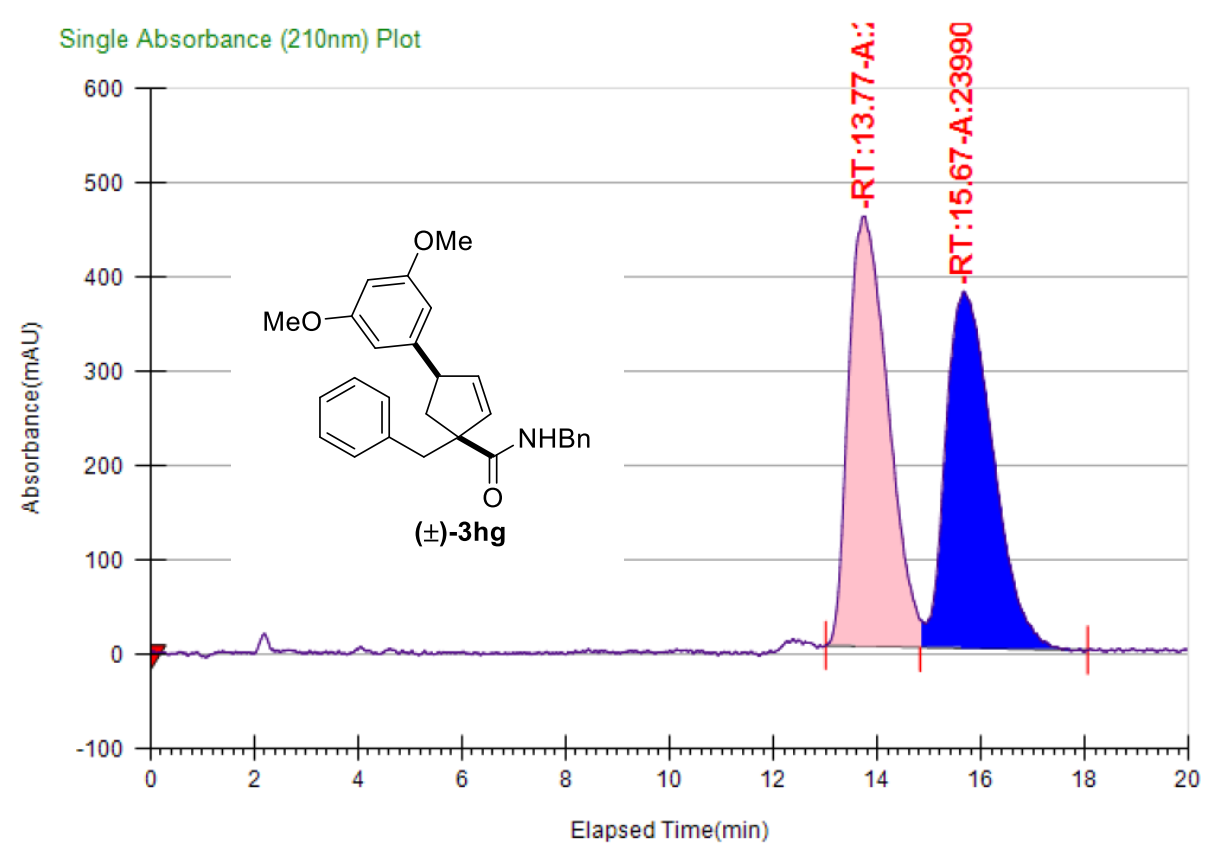

\begin{tabular}{|c|c|c|c|c|}
\hline Co-Solvent \% & Total Flow & Column & Co-Solvent & Back Pressure \\
\hline 10 & 4 & IC & $\mathrm{MeOH}$ & 150 \\
\hline Peak \# & Ret. Time & Area & Area $\%$ & \\
\hline 1 & $13.58 \mathrm{~min}$ & 1045.435 & 2.3343 & \\
\hline 2 & $15.21 \mathrm{~min}$ & 43740.9297 & \begin{tabular}{l|l|}
7 & 97.6657
\end{tabular} & \\
\hline
\end{tabular}

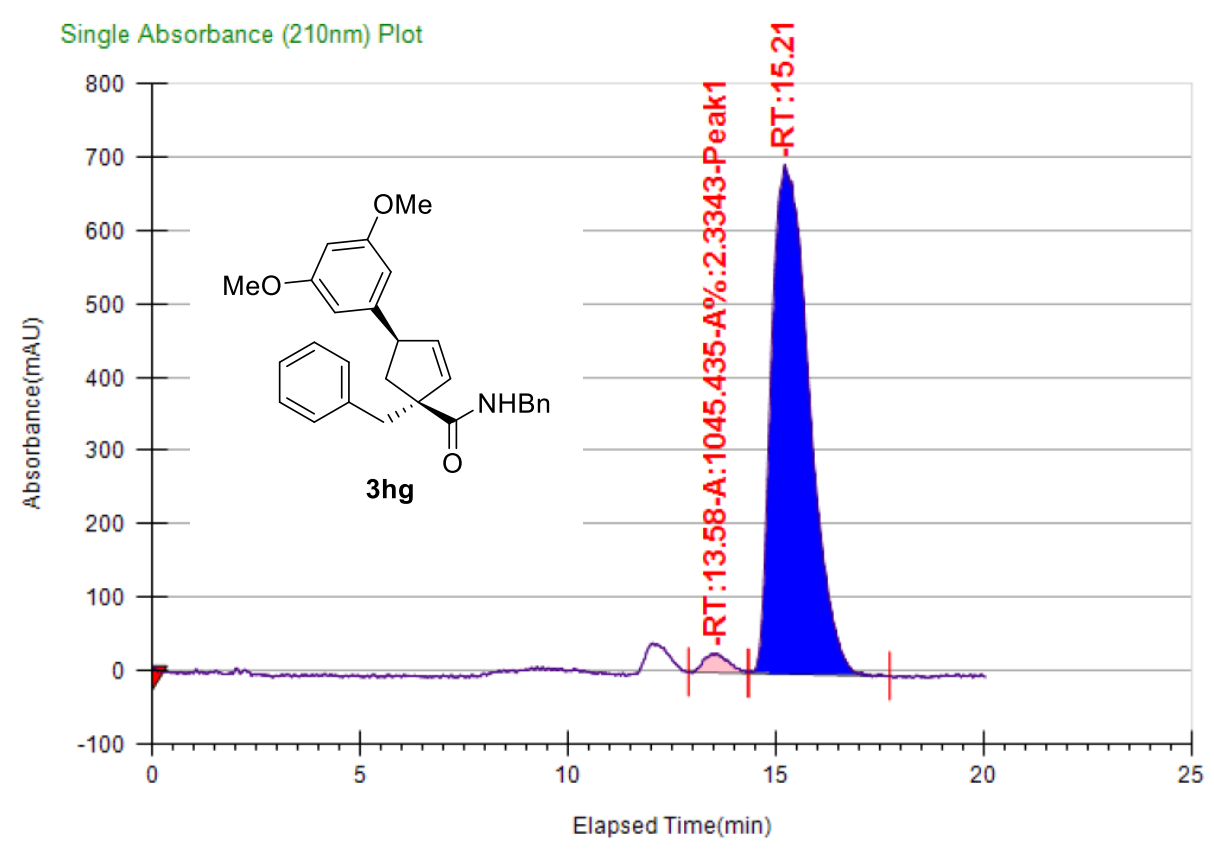




\begin{tabular}{|c|c|c|c|c|}
\hline Co-Solvent \% & Total Flow & Column & Co-Solvent & Back Pressure \\
\hline 5 & 4 & IA & $\mathrm{MeOH}$ & 150 \\
\hline Peak \# & Ret. Time & Area & Area $\%$ & \\
\hline 1 & $11.34 \mathrm{~min}$ & 15178.6184 & \begin{tabular}{|l|l|}
4 & 48.1388 \\
\end{tabular} & \\
\hline 2 & $12.72 \mathrm{~min}$ & 16352.3572 & \begin{tabular}{|l|l|}
2 & 51.8612
\end{tabular} & \\
\hline
\end{tabular}
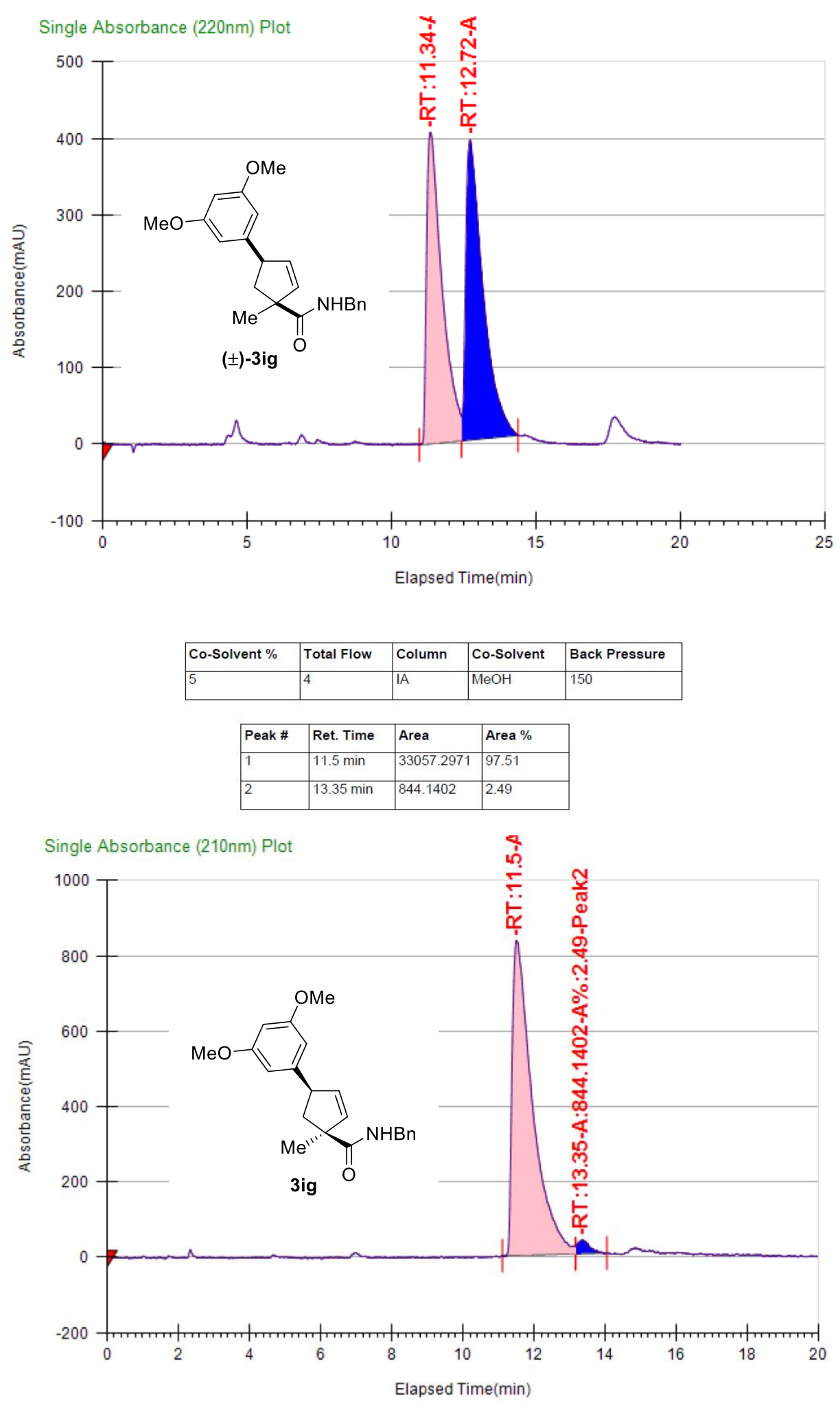


\begin{tabular}{|c|c|c|c|c|}
\hline Co-Solvent \% & Total Flow & Column & Co-Solvent & Back Pressure \\
\hline 7.0018 & 3.999 & AD-H & $\mathrm{MeOH}$ & 150 \\
\hline Peak \# & Ret. Time & Area & Area $\%$ & \\
\hline 1 & $9.57 \mathrm{~min}$ & 44260.8175 & \begin{tabular}{l|l|}
5 & 50.2286
\end{tabular} & \\
\hline 2 & $14.74 \mathrm{~min}$ & 43857.8953 & \begin{tabular}{|l|l|}
3 & 49.7714 \\
\end{tabular} & \\
\hline
\end{tabular}
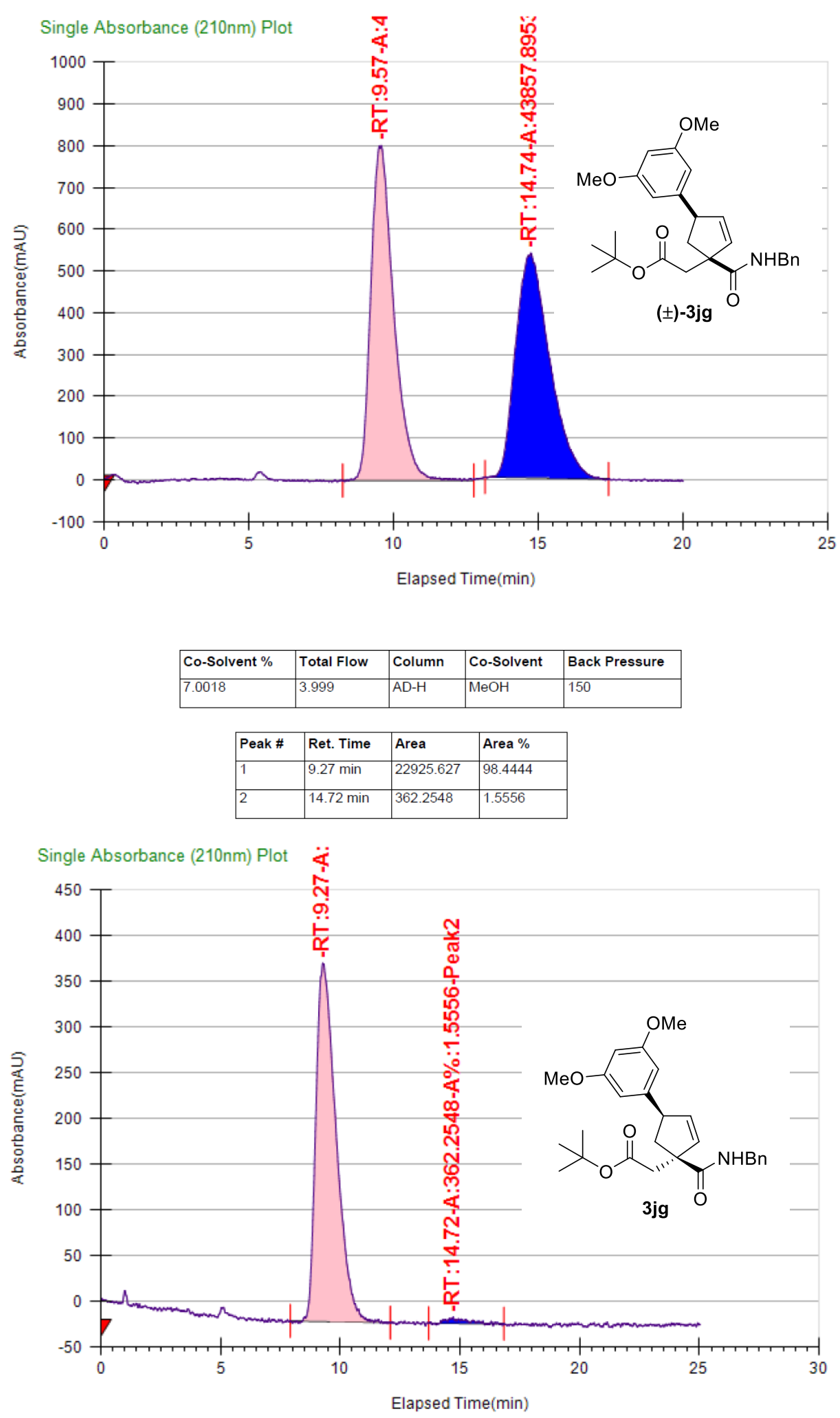


\begin{tabular}{|c|c|c|c|c|}
\hline Co-Solvent \% & Total Flow & Column & Co-Solvent & Back Pressure \\
\hline 5 & 4 & IB & $\mathrm{MeOH}$ & 150 \\
\hline Peak \# & Ret. Time & Area & Area \% & \\
\hline 1 & $15.07 \mathrm{~min}$ & 45422.6819 & \begin{tabular}{|l|l} 
& 51.4285
\end{tabular} & \\
\hline 2 & $18.43 \mathrm{~min}$ & 42899.3838 & \begin{tabular}{|l|l|}
3 & 48.5715 \\
\end{tabular} & \\
\hline
\end{tabular}

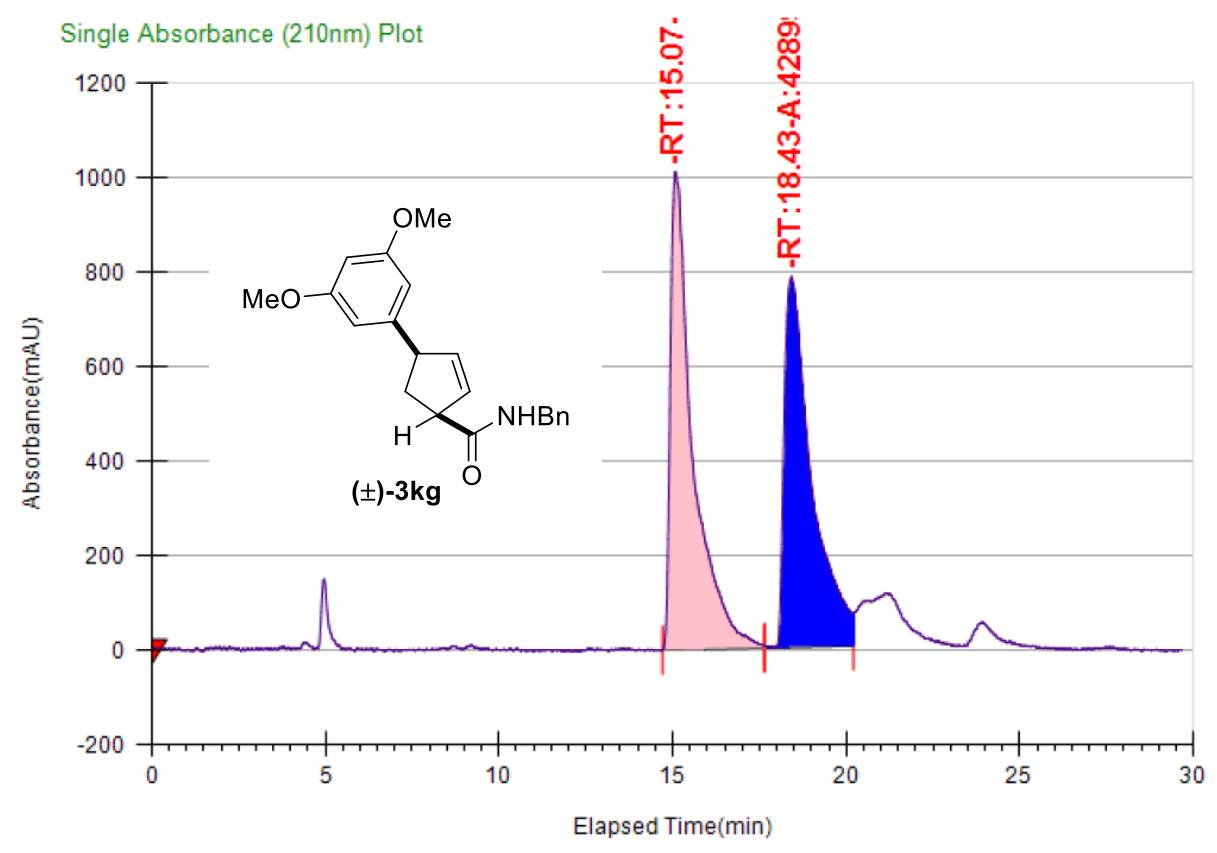

\begin{tabular}{|c|c|c|c|c|}
\hline Co-Solvent \% & Total Flow & Column & Co-Solvent & Back Pressure \\
\hline 5 & 4 & IB & $\mathrm{MeOH}$ & 150 \\
\hline Peak \# & Ret. Time & Area & Area $\%$ & \\
\hline \begin{tabular}{|l|}
1 \\
1
\end{tabular} & $17.13 \mathrm{~min}$ & 961.1779 & 2.2063 & \\
\hline 2 & $20.27 \mathrm{~min}$ & 42603.2374 & \begin{tabular}{|l|l|}
4 & 97.7937
\end{tabular} & \\
\hline
\end{tabular}

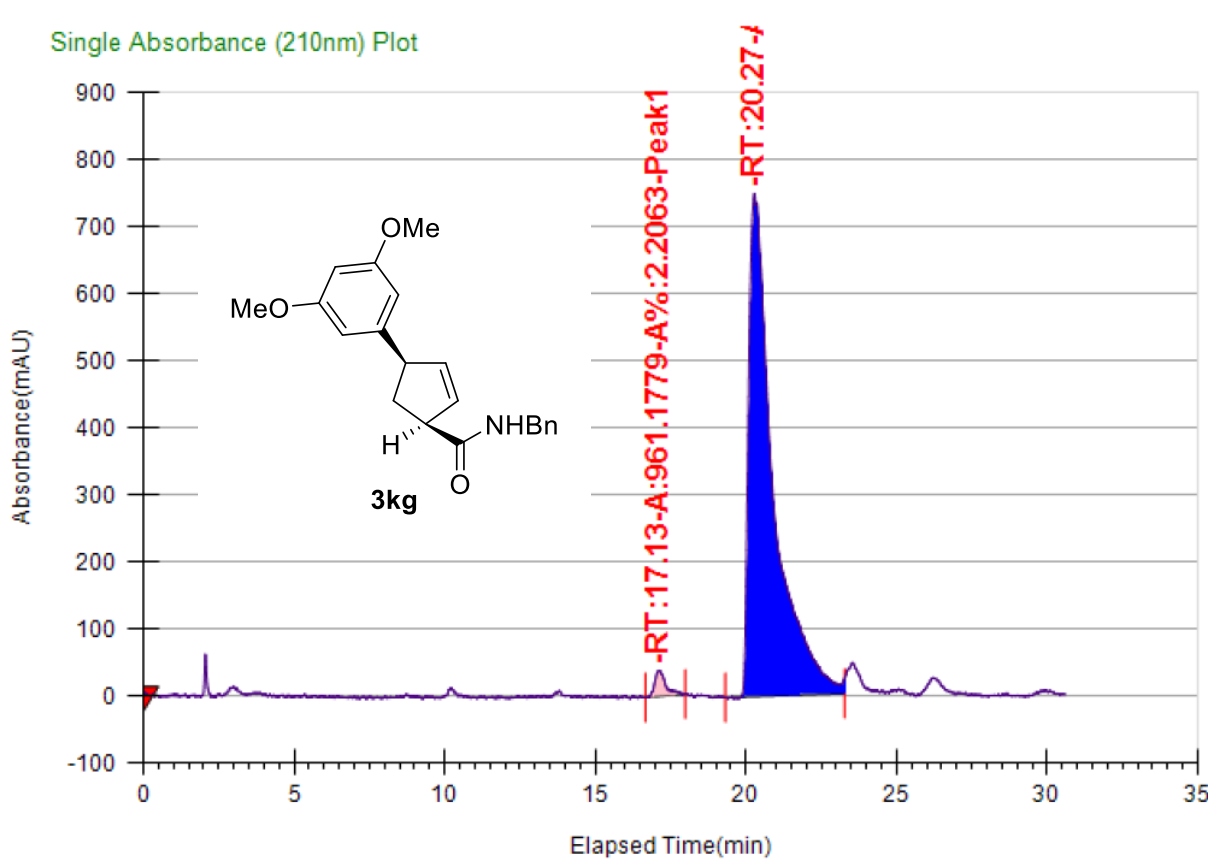




\begin{tabular}{|c|c|c|c|c|}
\hline Co-Solvent \% & Total Flow & Column & Co-Solvent & Back Pressure \\
\hline 10 & 4 & AD-H & $\mathrm{MeOH}$ & 150 \\
\hline Peak \# & Ret. Time & Area & Area \% & \\
\hline 1 & $5.41 \mathrm{~min}$ & 27477.7412 & \begin{tabular}{|l|l|}
2 & 50.0394
\end{tabular} & \\
\hline 2 & $7.89 \mathrm{~min}$ & 27434.5152 & \begin{tabular}{l|l}
2 & 49.9606
\end{tabular} & \\
\hline
\end{tabular}

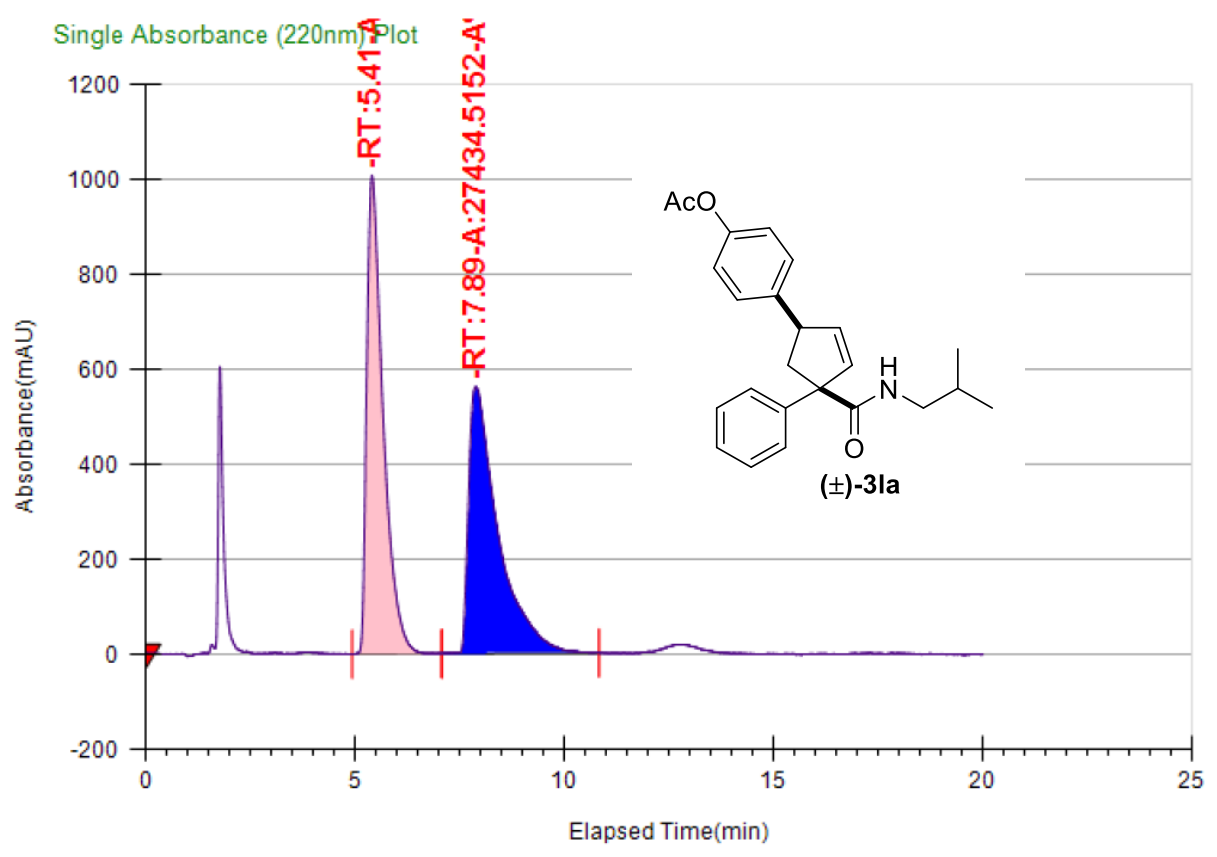

\begin{tabular}{|c|c|c|c|c|}
\hline Co-Solvent \% & Total Flow & Column & Co-Solvent & Back Pressure \\
\hline 10 & 4 & AD-H & $\mathrm{MeOH}$ & 150 \\
\hline Peak \# & Ret. Time & Area & Area \% & \\
\hline 1 & $5.55 \mathrm{~min}$ & 2052.4414 & 9.0847 & \\
\hline 2 & $7.92 \mathrm{~min}$ & 20539.7387 & \begin{tabular}{|l|l|}
7 & 90.9153
\end{tabular} & \\
\hline
\end{tabular}

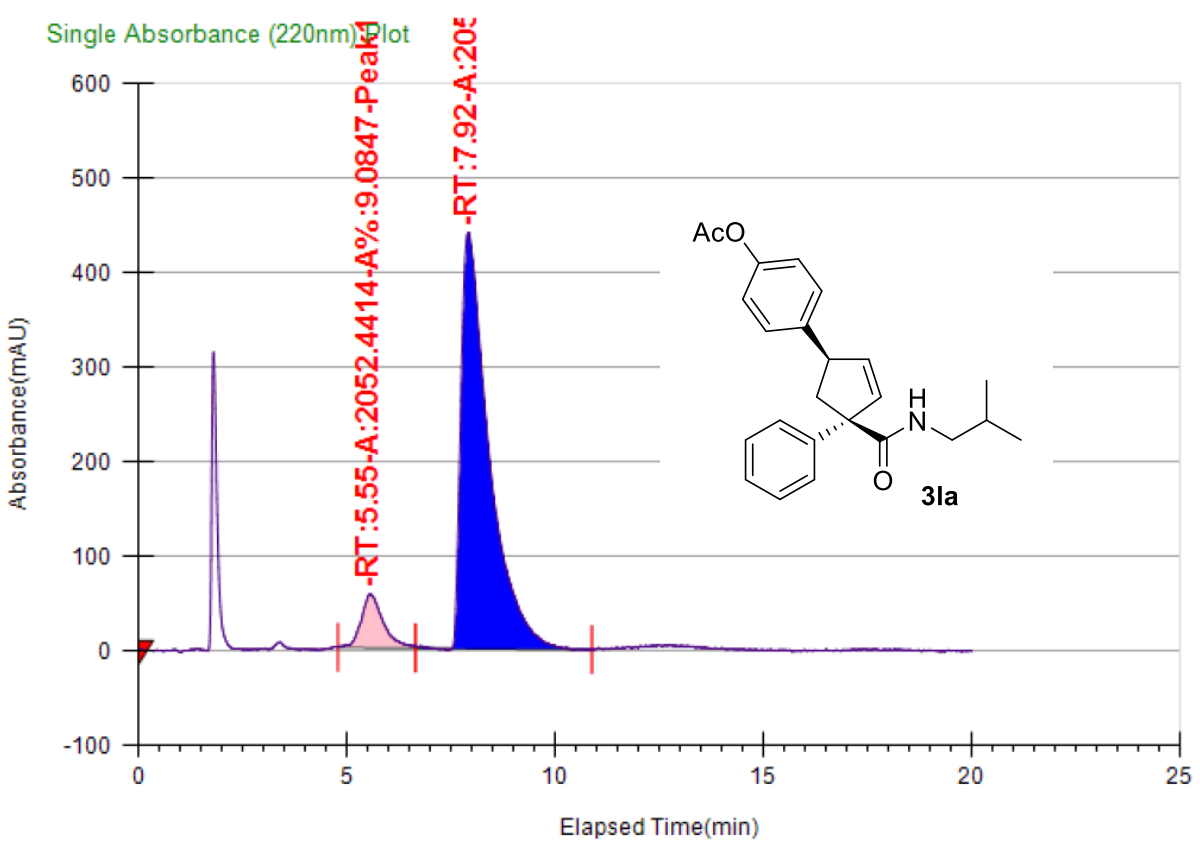




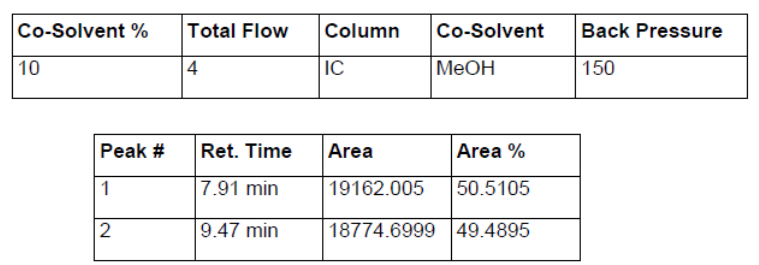
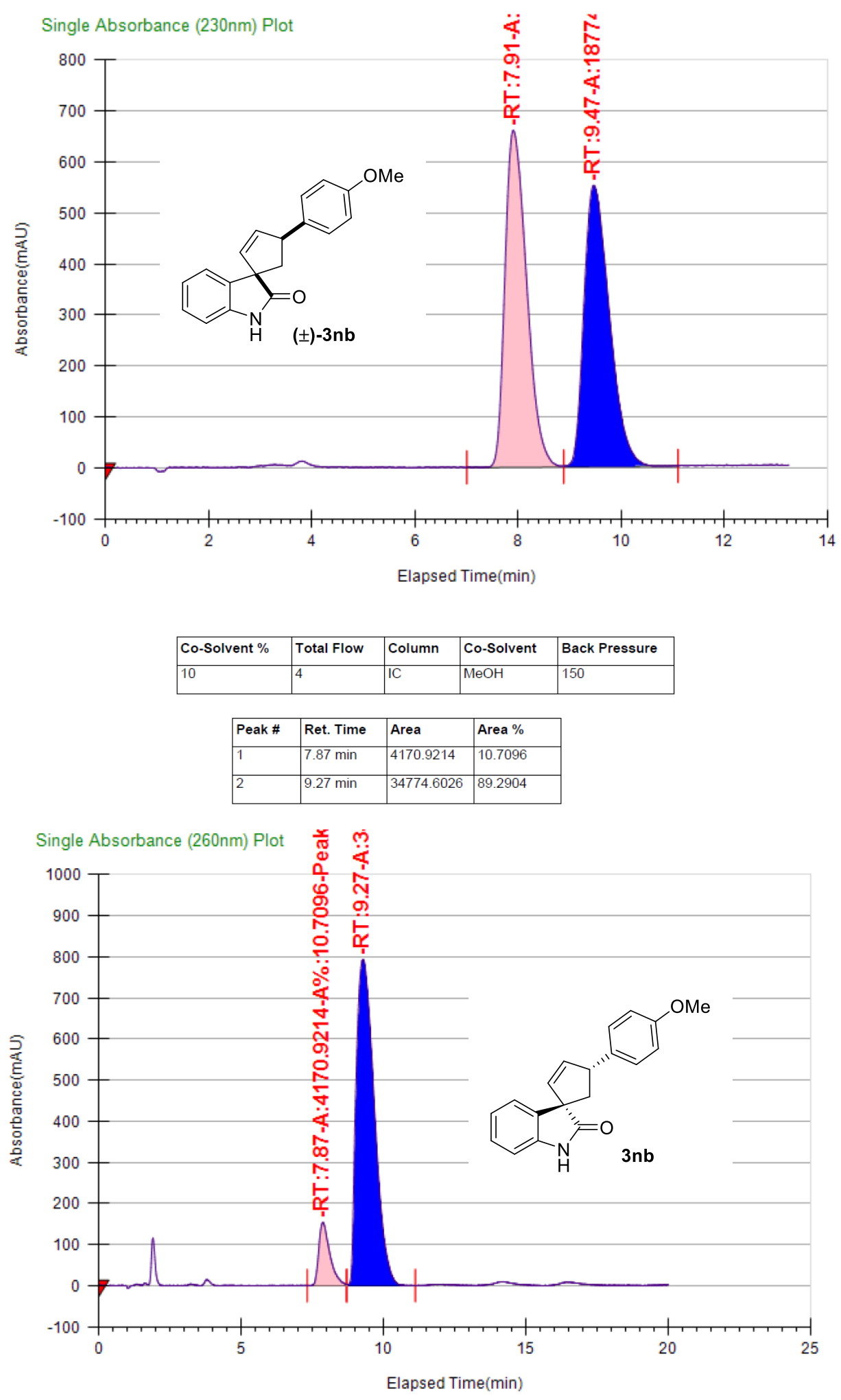


\begin{tabular}{|c|c|c|c|c|}
\hline Co-Solvent \% & Total Flow & Column & Co-Solvent & Back Pressure \\
\hline 10 & 4 & IA & $\mathrm{MeOH}$ & 150 \\
\hline Peak \# & Ret. Time & Area & Area $\%$ & \\
\hline 1 & $4.83 \mathrm{~min}$ & 27557.9412 & \begin{tabular}{|l|l|}
2 & 49.7168
\end{tabular} & \\
\hline 2 & $5.65 \mathrm{~min}$ & 27871.8439 & \begin{tabular}{l|l}
9 & 50.2832
\end{tabular} & \\
\hline
\end{tabular}

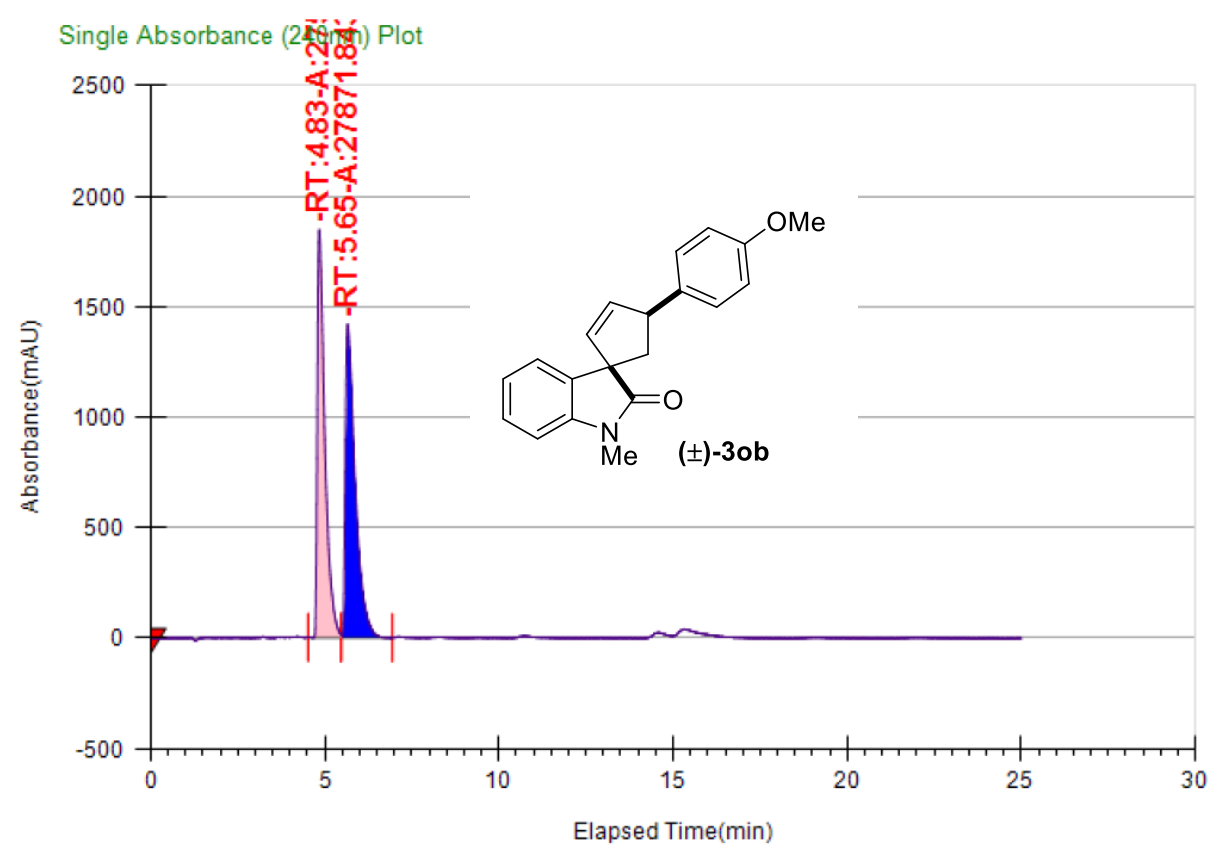

\begin{tabular}{|c|c|c|c|c|}
\hline Co-Solvent \% & Total Flow & Column & Co-Solvent & Back Pressure \\
\hline 10 & 4 & IA & $\mathrm{MeOH}$ & 150 \\
\hline Peak \# & Ret. Time & Area & Area $\%$ & \\
\hline \begin{tabular}{|l}
1 \\
1
\end{tabular} & $4.57 \mathrm{~min}$ & 4227.7166 & 10.5012 & \\
\hline 2 & $5.13 \mathrm{~min}$ & 36031.7953 & \begin{tabular}{|l|l|}
3 & 89.4988 \\
\end{tabular} & \\
\hline
\end{tabular}

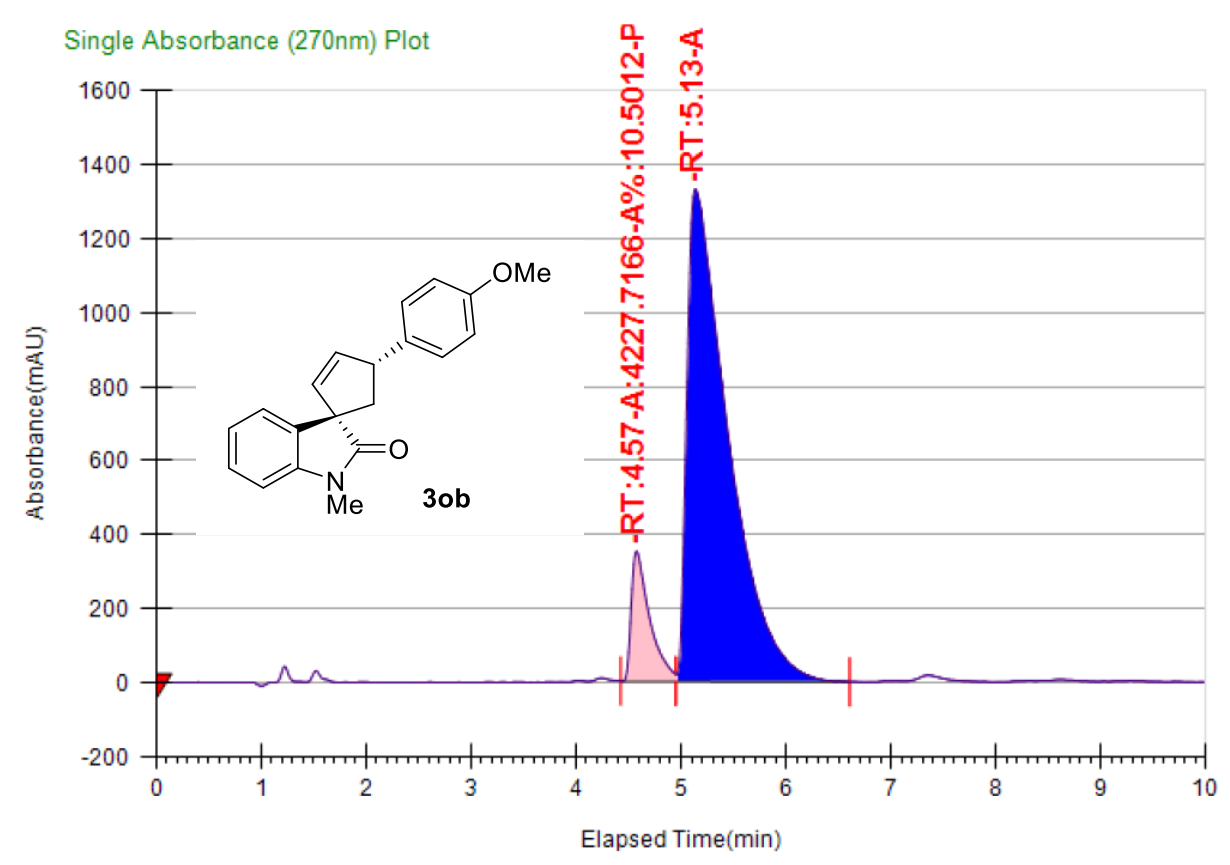




\begin{tabular}{|c|c|c|c|c|}
\hline Co-Solvent \% & Total Flow & Column & Co-Solvent & Back Pressure \\
\hline 5 & 4 & IC & $\mathrm{MeOH}$ & 150 \\
\hline Peak \# & Ret. Time & Area & Area \% & \\
\hline 1 & $7.59 \mathrm{~min}$ & 36209.6838 & \begin{tabular}{|l|l|}
3 & 48.9303
\end{tabular} & \\
\hline 2 & $8.89 \mathrm{~min}$ & 37792.9292 & \begin{tabular}{l|l}
2 & 51.0697
\end{tabular} & \\
\hline
\end{tabular}

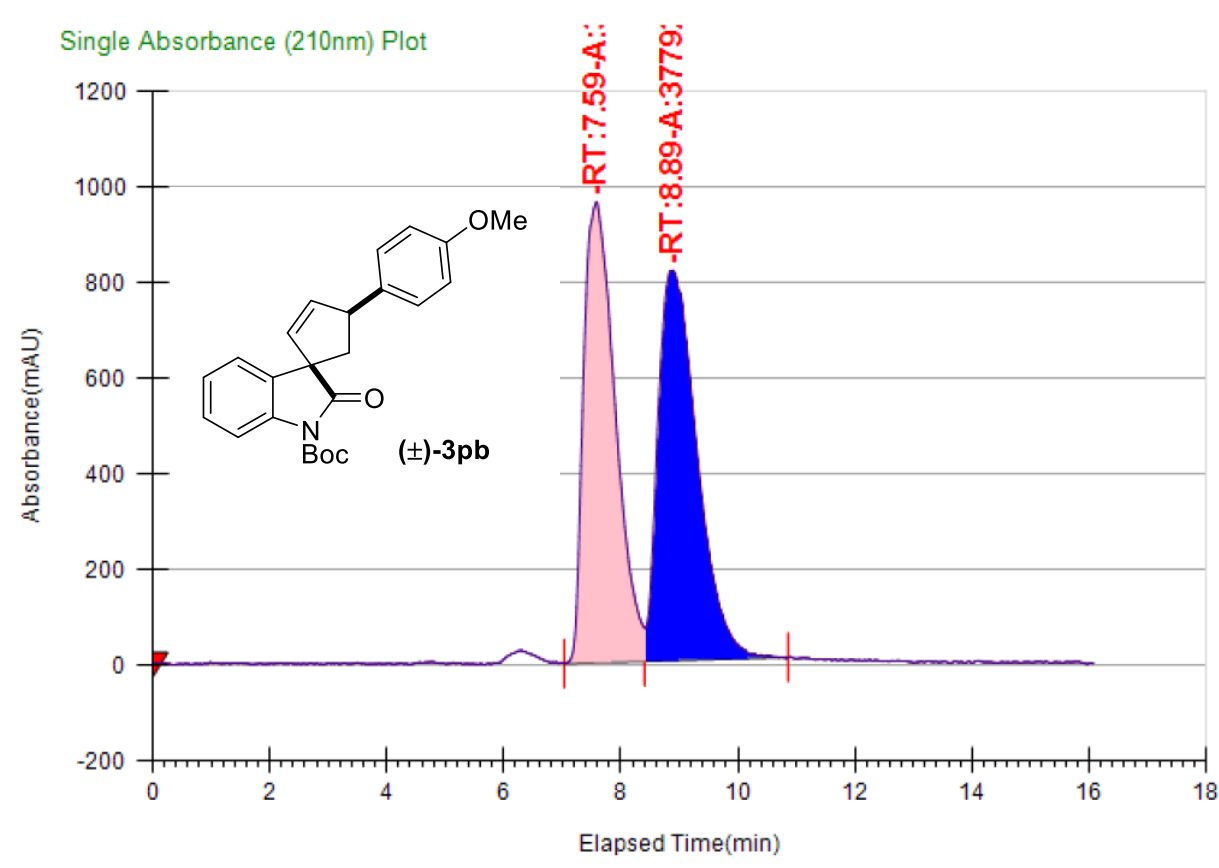

\begin{tabular}{|c|c|c|c|c|}
\hline Co-Solvent \% & Total Flow & Column & Co-Solvent & Back Pressure \\
\hline 5 & 4 & IC & $\mathrm{MeOH}$ & 150 \\
\hline Peak \# & Ret. Time & Area & Area $\%$ & \\
\hline 1 & $7.65 \mathrm{~min}$ & 5461.8018 & 12.7719 & \\
\hline 2 & $8.89 \mathrm{~min}$ & \begin{tabular}{|l|}
37302.2448 \\
\end{tabular} & \begin{tabular}{|l|l|}
3 & 87.2281 \\
\end{tabular} & \\
\hline
\end{tabular}

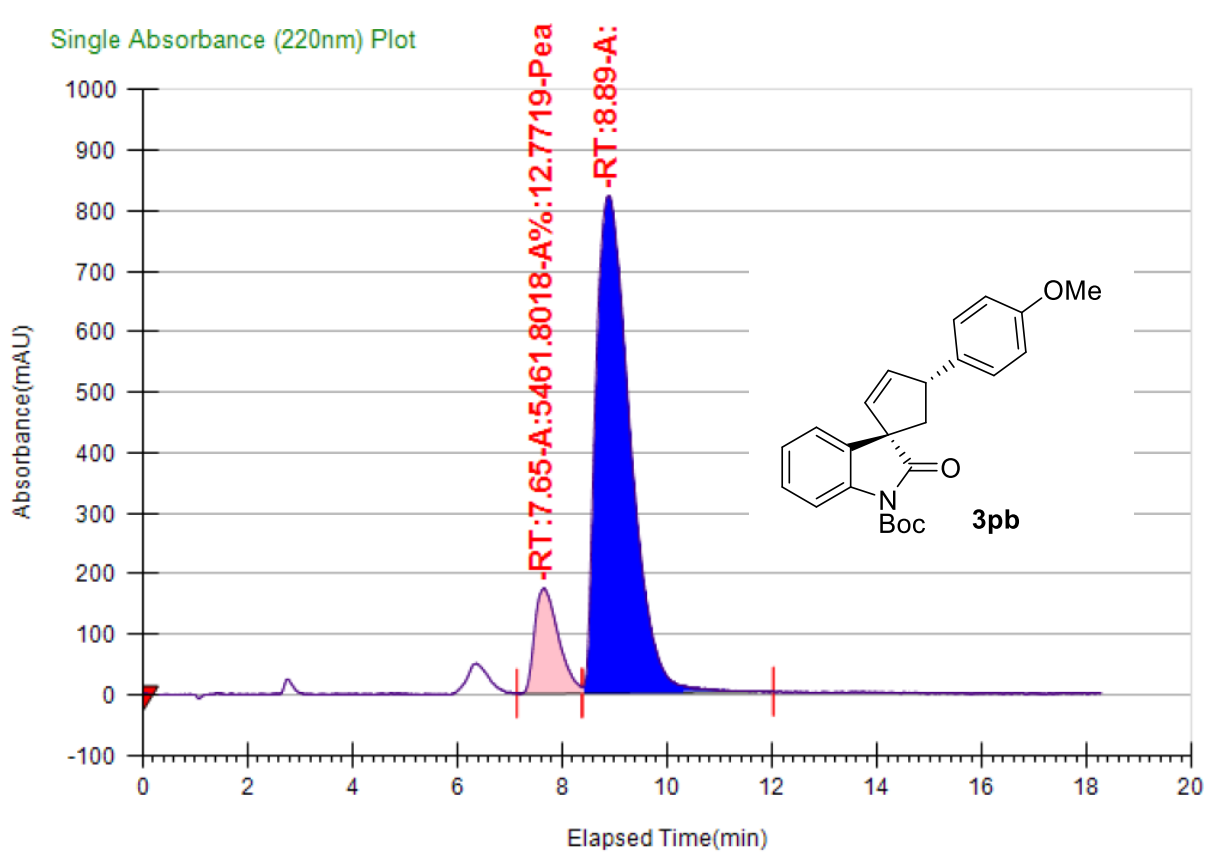




\begin{tabular}{|c|c|c|c|c|}
\hline Co-Solvent \% & Total Flow & Column & Co-Solvent & Back Pressure \\
\hline 10 & 4 & AD-H & $\mathrm{MeOH}$ & 150 \\
\hline Peak \# & Ret. Time & Area & Area $\%$ & \\
\hline 1 & $3.22 \mathrm{~min}$ & 26917.8632 & \begin{tabular}{l|l}
27.007 \\
\end{tabular} & \\
\hline 2 & $5.56 \mathrm{~min}$ & 30345.6623 & \begin{tabular}{|l|l|}
3 & 52.993 \\
\end{tabular} & \\
\hline
\end{tabular}

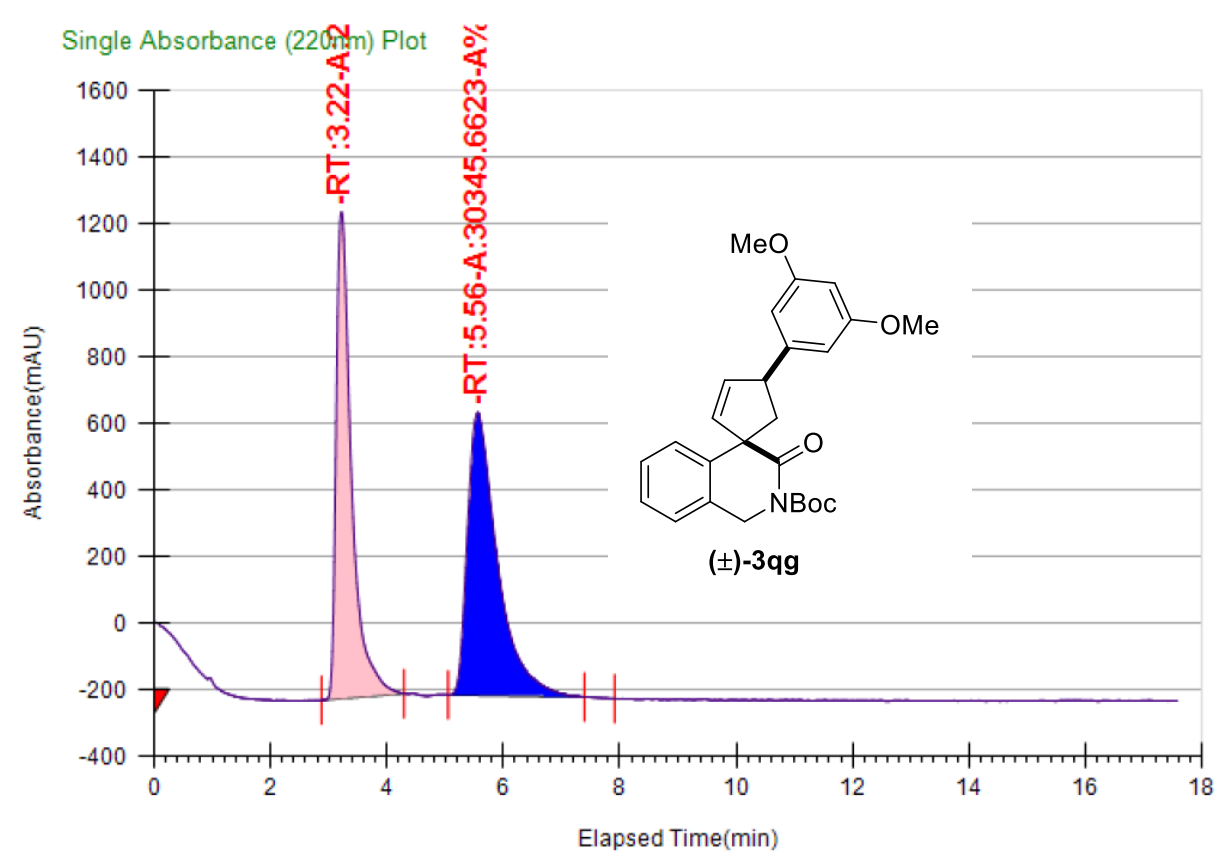

\begin{tabular}{|c|c|c|c|c|}
\hline Co-Solvent \% & Total Flow & Column & Co-Solvent & Back Pressure \\
\hline 10 & 4 & AD-H & $\mathrm{MeOH}$ & 150 \\
\hline Peak \# & Ret. Time & Area & Area \% & \\
\hline 1 & $3.31 \mathrm{~min}$ & 20019.4112 & \begin{tabular}{|l|l|}
2 & 95.7924
\end{tabular} & \\
\hline 2 & $5.84 \mathrm{~min}$ & 879.3466 & 4.2076 & \\
\hline
\end{tabular}

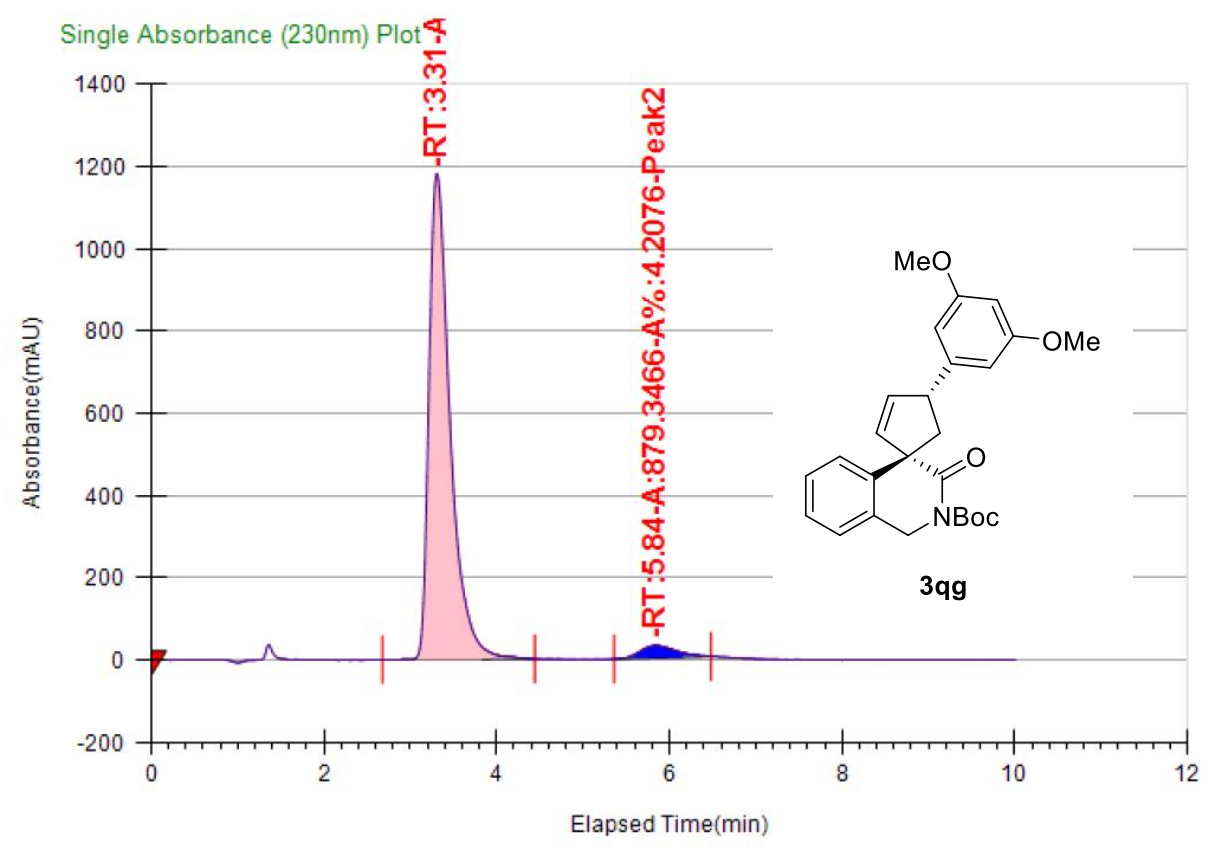




\section{Crystallographic data for 4 and 5}
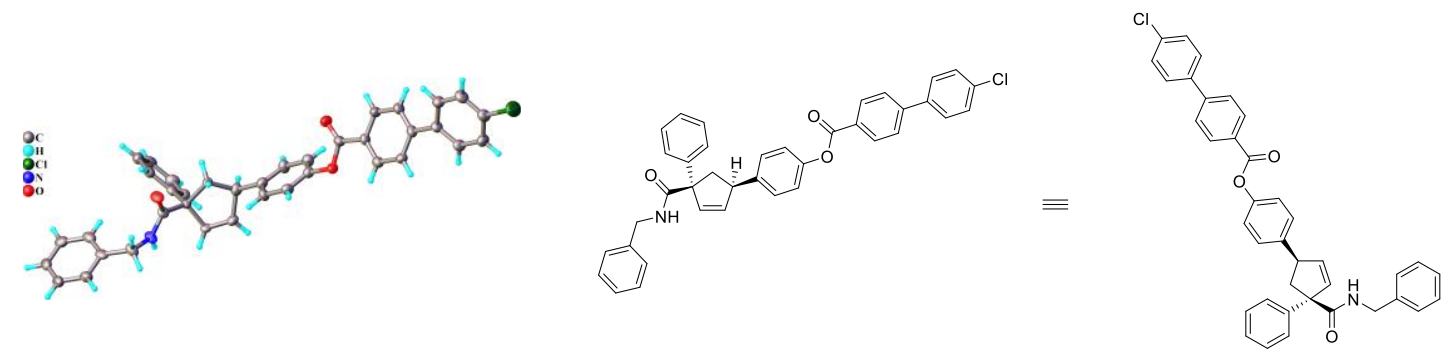

Table 1. Crystal data and structure refinement for 4 .

Empirical formula

Formula weight

Temperature

Wavelength

Crystal system

Space group

Unit cell dimensions

Volume

$\mathrm{Z}$

Density (calculated)

Absorption coefficient

$\mathrm{F}(000)$

Crystal size

$\theta$ range for data collection

Index ranges

Reflections collected

Independent reflections

Completeness to $\theta=67.684^{\circ}$

Absorption correction

Max. and min. transmission

Refinement method

Data / restraints / parameters

Goodness-of-fit on $F^{2}$

Final $R$ indices $[\mathrm{I}>2 \sigma(\mathrm{I})]$

$R$ indices (all data)

Absolute structure parameter

Largest diff. peak and hole
$\mathrm{C}_{38} \mathrm{H}_{30} \mathrm{ClNO}_{3}$

584.08

100.00(10) K

$1.54184 \AA$

Triclinic

$P 1$

$\mathrm{a}=9.9640(4) \AA$

$\alpha=99.233(3)^{\circ}$.

$\mathrm{b}=10.9528(5) \AA$

$\beta=92.081(3)^{\circ}$.

$\mathrm{c}=27.8818(10) \AA$

$\gamma=102.422(4)^{\circ}$.

2925.2(2) $\AA^{3}$

4

$1.326 \mathrm{Mg} / \mathrm{m}^{3}$

$1.471 \mathrm{~mm}^{-1}$

1224

$0.688 \times 0.079 \times 0.024 \mathrm{~mm}^{3}$

4.196 to $75.824^{\circ}$.

$-12 \leq \mathrm{h} \leq 9,-13 \leq \mathrm{k} \leq 13,-34 \leq 1 \leq 33$

14886

$14886\left[R_{\text {int }}=\mathrm{n} / \mathrm{a}\right]$

$99.7 \%$

Gaussian

1.000 and 0.389

Full-matrix least-squares on $\mathrm{F}^{2}$

14886 / 3 / 1550

1.003

$R_{1}=0.0736, w R_{2}=0.1913$

$R_{1}=0.0853, w R_{2}=0.2011$

0.054(11)

0.748 and -0.616 e. $\AA^{-3}$ 

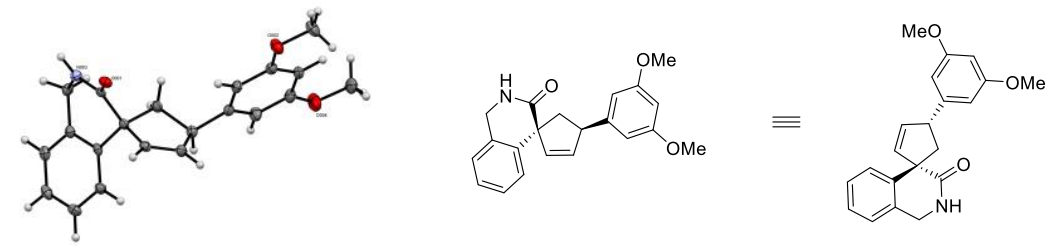

Table 2. Crystal data and structure refinement for 5.

Empirical formula

Formula weight

Temperature

Wavelength

Crystal system

Space group

Unit cell dimensions

Volume

Z

Density (calculated)

Absorption coefficient

$\mathrm{F}(000)$

Crystal size

$\theta$ range for data collection

Index ranges

Reflections collected

Independent reflections

Completeness to $\theta=67.684^{\circ}$

Absorption correction

Max. and min. transmission

Refinement method

Data / restraints / parameters

Goodness-of-fit on $F^{2}$

Final $R$ indices $[\mathrm{I}>2 \sigma(\mathrm{I})]$

$R$ indices (all data)

Absolute structure parameter

Extinction coefficient

Largest diff. peak and hole
$\mathrm{C}_{21} \mathrm{H}_{21} \mathrm{NO}_{3}$

335.39

99.99(10) K

$1.54184 \AA$

Monoclinic

C2

$$
\begin{array}{ll}
\mathrm{a}=15.3040(4) \AA & \alpha=90^{\circ} . \\
\mathrm{b}=7.09902(15) \AA & \beta=108.778(3)^{\circ} . \\
\mathrm{c}=16.7657(4) \AA & \gamma=90^{\circ} .
\end{array}
$$

1724.53(7) $\AA^{3}$

4

$1.292 \mathrm{Mg} / \mathrm{m}^{3}$

$0.693 \mathrm{~mm}^{-1}$

712

$0.751 \times 0.230 \times 0.085 \mathrm{~mm}^{3}$

5.574 to $73.611^{\circ}$.

$-18 \leq \mathrm{h} \leq 17,-8 \leq \mathrm{k} \leq 8,-19 \leq 1 \leq 20$

7858

$3270\left[R_{\text {int }}=0.0198\right]$

$99.8 \%$

Gaussian

1.000 and 0.600

Full-matrix least-squares on $F^{2}$

$3270 / 1 / 229$

1.043

$R_{1}=0.0280, w R_{2}=0.0730$

$R_{1}=0.0290, w R_{2}=0.0741$

0.01(9)

$0.00049(17)$

0.198 and -0.154 e. $\AA^{-3}$ 